\title{
Molecular Mechanisms of Tau Protein Aggregation Inhibition
}

\author{
Dissertation \\ Zur Erlangung des mathematisch-naturwissenschaftlichen Doktorgrades \\ "Doctor rerum naturalium" \\ der Georg-August-Universität Göttingen
}

vorgelegt von

Elias Akoury

aus Kattine ou Hidab, Libanon

Göttingen 2013 
Mitglied des Betreuungsausschusses (Referent): Prof. Dr. Markus Zweckstetter

NMR-basierte Strukturbiologie, Max-Planck-Institut für biophysikalische Chemie

Mitglied des Betreuungsausschusses (Referentin): Prof. Dr. Marina Bennati

Elektronenspinresonanz-Spektroskopie, Max-Planck-Institut für biophysikalische Chemie

Mitglied des Betreuungsausschusses: Prof. Dr. Tiago Fleming Outeiro

Abteilung für Neurodegeneration und Neuroestaurationsforschung, Universitätsmedizin Göttingen

Mitglied der Prüfungskommission: Prof. Dr. Kai Tittmann

Abteilung für Bioanalytik des Albrecht-von-Haller-Instituts, Georg-August-Universität Göttingen

Mitglied der Prüfungskommission: Dr. Lars Thorsten Kuhn

Abteilung für NMR Spektroskopie, Europäisch Neurowissenschaftliche Institut Göttingen

Mitglied der Prüfungskommission: Dr. Adam Lange

NMR-basierte Strukturbiologie, Max-Planck-Institut für biophysikalische Chemie

Tag der mündlichen Prüfung: Montag, der 30. September 2013 


\begin{abstract}
Affidavit
I hereby declare that the thesis "Molecular Mechanisms of Tau Protein Aggregation Inhibition" has been written independently and with no other sources and aids than quoted.
\end{abstract}

\author{
Elias Akoury \\ Göttingen, the $26^{\text {th }}$ of August 2013
}




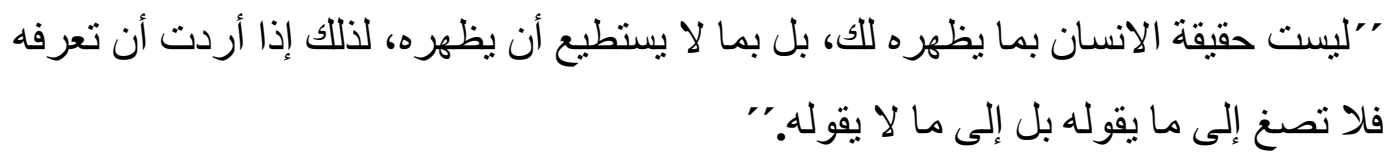

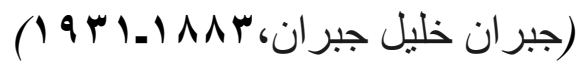

"'An experiment is a question which science poses to Nature and a measurement is the recording of Nature's answer."

(Max Karl Ernst Ludwig Planck, 1858-1947)

'Zwar weiß ich viel, doch möchte ich alles wissen."

(Johann Wolfgang von Goethe, 1749-1832)

"Rien ne se perd, rien ne se crée, tout se transforme."

(Antoine-Laurent de Lavoisier, 1743-1794) 


\section{Dedication}

I dedicate this thesis to my parents who have been a great source of inspiration and support. This thesis is also dedicated to Prof. Dr. Stefan Berger who encouraged me to build my motivation towards the world of NMR. 


\section{Acknowledgements}

This life changing journey has come to an end, and obviously, the success of any event depends largely on the encouragement and guidelines of many persons that, with no doubts, must be mentioned.

I would like to express my deepest appreciation and full respect to my supervisor Prof. Dr. Markus Zweckstetter for his priceless support, infinite patience, and endless motivation in conducting scientific research. The great enthusiasm and full effort he invested in guiding and advising lead to achievements in publishing the manuscripts and writing the thesis. I am deeply grateful to Prof. Dr. Christian Griesinger for stimulating discussions, positive feedbacks and professional research environment at the department of NMR-based Structural Biology.

I owe huge debt of gratitude to the PhD thesis committee members Prof. Dr. Marina Bennati and Prof. Dr. Tiago Fleming Outeiro for their tremendous support and insightful guidance. Special thanks go to Prof. Dr. Kai Tittmann, Dr. Lars Thorsten Kuhn, and Dr. Adam Lange for kindly participating in the examination committee.

I appreciate the guidance of the GGNB offices and Petra Breiner for all the paperwork.

Our collaborators deserve a special acknowledgment for productive scientific discussions and excellent sample preparations over the years. Big respects are owned to Prof. Dr. Eckhard Mandelkow, Dr. Jacek Biernat, and Dr. Marcus Pickhardt for the Tau protein project; to Dr. Chad Dickey and Bryce Nordhues for the Heat shock protein project.

I particularly would like to thank Sheng Qi Xiang who was my ultimate reference to NMR methodology at all times and an experienced supporter in my responsibility of the $900 \mathrm{MHz}$ spectrometer. I take the opportunity to express my thankfulness to the colleagues Luis Fonseca, Aldo Camacho, Xuejun Yao, and Rakhi Bajaj who have been compassionate and tolerant at different aspects. 
I would like to acknowledge the support of Dr. Francesca Munari, Dr. Nasrollah Rezaei-Ghaleh, Dr. Nils-Alexander Lakomek and Dr. Piotr Wysoczanski for outstanding scientific consultations; Dr. Michal Gajda for SAXS analysis, Dr. Soraya Pornsuwan for EPR analysis, and Romina Hofele for intensive mass spectrometric discussions. I am delighted to recognize the extensive research on Tau conducted by my predecessors Dr. Marco Mukrasch, Dr. Daniela Fischer and Dr. Stefan Bibow and current colleagues Dr. Martin Schwalbe and Harindranath Kadavath.

I have experienced warm encouragements, constructive comments and appreciated friendships from previous and current cowokers of MPIBPC and MPIDS: Dr. Lisandro Falomir, Dr. Anthony de Vries, Dr. Zrinka Gattin, Dr. Dominika Czernik, Dr. Michelle Gralle Botelho, Dr. Julia Preobraschenski, Dr. Carmen Sanchez Moreno, Elisa Turriani, Florencia Gillanders, Caroline Behrens, Sebastián Andrés Díaz, Romina Hofele, Mario Modena, Benjamin Schulz, Birte Riechers, Julie Murison and Quentin Brosseau.

I wish to express my sincere gratitude for the encouragement and inspiration I have received from Caroline Lehner, Carolina Sánchez Rico, Annika Otto, Bernadett Zielonka, Isabelle Hellwig, and Lynn Sampson.

My deepest appreciation goes to the persons who are so close no matter how far, my friends and family Chad Akoury, Hady Barhouch, Roy Obeid, Chantal Souaid Mchantaf, Pauline El Khoury Barghachieh, Marie-Noel Zeenny and Nicolas Saadeh.

I am grateful to my coach Michel Ferneini not only for he has implemented in me the perseverance of paddling a kayak forward, but also for paddling through the challenges of life. I owe huge respects to Elie Bassil, to the Scout association at the Antonine Sisters School, and Saint Elias church who actively supported my determination to find and realize my potentials and self-determination.

Last but not least, I owe countless respects and love to my mother Claude Akoury, my father Antoine Akoury, and my two brothers Paul Akoury and Fady Akoury, who have been a source of endless love with unlimited support and inspiration throughout my life. 


\section{Publication List}

The thesis is based on the following publications:

Inhibition of Tau Filament Formation by Conformational Modulation.

Akoury E, Gajda MJ, Pickhardt M, Biernat J, Soraya P, Griesinger C, Mandelkow E, Zweckstetter M. J Am Chem Soc. 2013 Jan 29;135(7):2853-62.

Mechanistic Basis of Phenothiazine-Driven inhibition of Tau Aggregation.

Akoury E, Pickhardt M, Gajda MJ, Biernat J, Mandelkow E, Zweckstetter M.

Angew. Chem. Int. Ed. Engl. 2013 Mar 18;52(12):3511-3515.

Imbalance of Hsp70 family variants fosters tau accumulation.

Jinwal UK, Akoury E, Abisambra JF, O'Leary JC 3rd, Thompson AD, Blair LJ, Jin Y, Bacon J, Nordhues BA, Cockman M, Zhang J, Li P, Zhang B, Borysov S, Uversky VN, Biernat J, Mandelkow E, Gestwicki JE, Zweckstetter M, Dickey CA. FASEB J. 2012 Dec 27. 


\begin{abstract}
Of all neurodegenerative diseases, Alzheimer's Disease is the most widespread dementia syndrome, exhibiting progressive memory loss and intellectual abilities. One of the pathological hallmarks of this disease is associated to the presence of abundant intracellular deposits of the Tau protein. Accumulation of these stable species is a multistep process that involves the formation of various transients. Understanding this key step may eventually enable to obstruct aggregation.

Tau protein is an intrinsically disordered protein, abundant in neuronal axons where it promotes and stabilizes microtubule assembly. Chapter 1 discusses the physiological function and pathological consequences of this protein and sheds light on the current Taubased research implemented in therapeutic strategies. This chapter also reviews the identification of inhibitors of Tau aggregation as potential disease-modifying drugs; with a detailed discussion of two organic compounds we have recently screened, Phthalocyanine Tetrasulfonate and Phenothiazine Methylene Blue. The same chapter then introduces the Heat shock proteins and their role in Tau clearance.

The chapters that follow report our research investigation of aggregation inhibitors and chaperonic partners of Tau where we used an integrated approach to detect possible assemblies of pathogenic conformational transitions. Chapter 2 represents the Phthalocyanine tetrasulfonate study and its inhibition of Tau filament formation by conformational modulation. Chapter 3 investigates the mechanistic basis of the phenothiazine-driven inhibition of Tau aggregation. Chapter 4, examines how the imbalance of Hsp70 family variants fosters Tau accumulation. As a final point, the significance of the biophysical techniques incorporated in the three published manuscripts are briefly discussed in the last part of Chapter 1.




\section{Table of Contents}

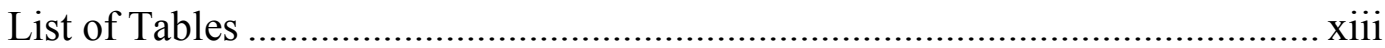

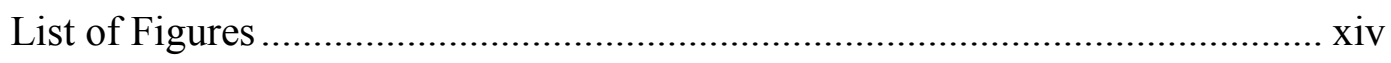

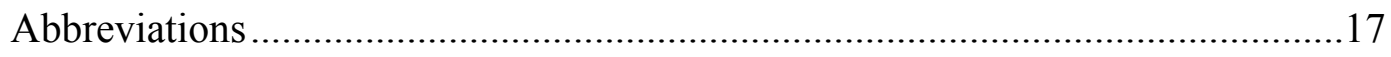

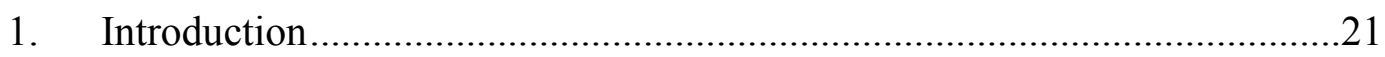

1.1 Protein Folding, Misfolding, and Aggregation ..............................................22

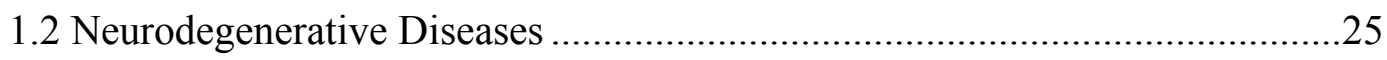

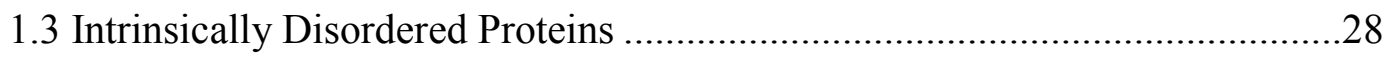

1.3.1 Structural Characteristics ………………………………….......28

1.3.2 Biological Function................................................................29

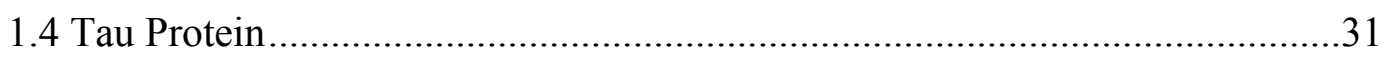

1.4.1 Localization, Function and Modifications ....................................31

1.4.2 Tau Pathology in Neurodegenerative Diseases............................37

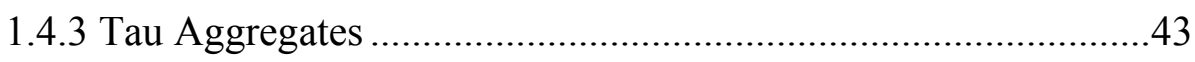

1.5 Tau-Based Therapeutic Strategies ................................................................48

1.5.1 Anti-phosphorylation Approaches ...............................................50

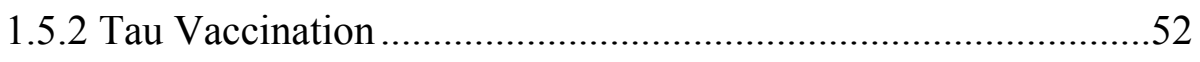

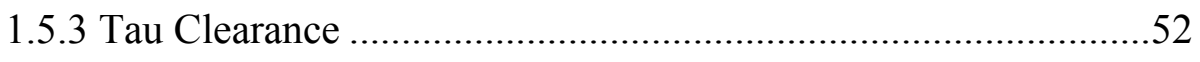

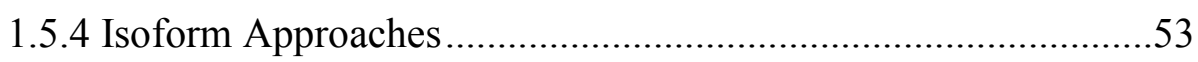

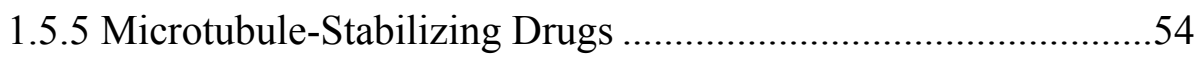

1.5.6 Anti-aggregation Strategies ......................................................55

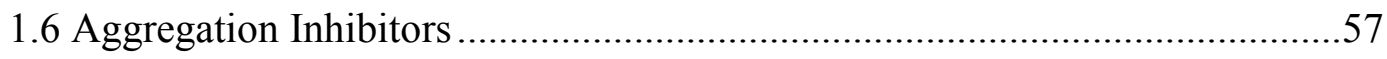

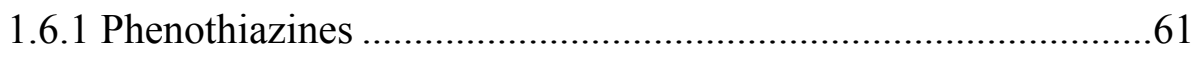

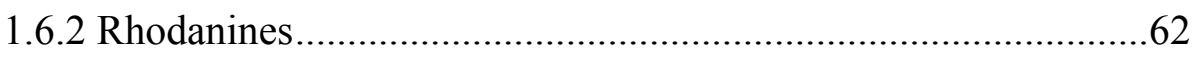

1.6.3 Phenylthiazolhydrazides ...........................................................63

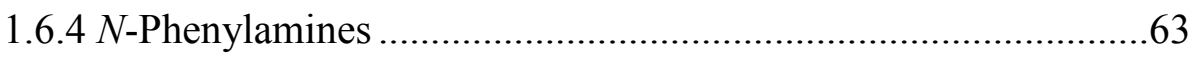

1.6.5 Anthraquinones ..............................................................................64 


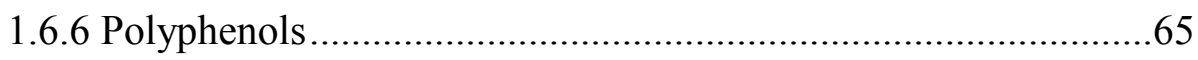

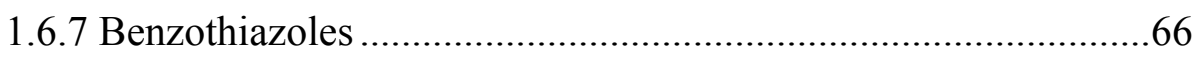

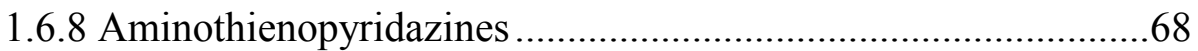

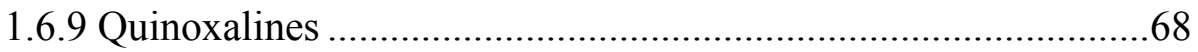

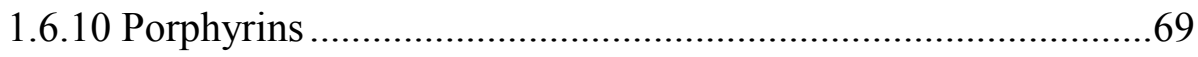

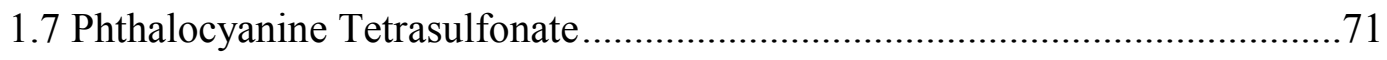

1.7.1 Physical and Chemical Properties.............................................. 71

1.7.2 Biological applications...............................................................

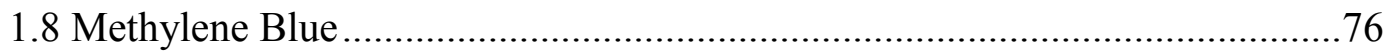

1.8.1 Physical, Chemical and Biochemical Properties .........................76

1.8.2 Biological applications..............................................................

1.8.3 The Impact of MB on Cysteine Oxidation....................................

1.8.4 MB and Protein Sulfenic Acids .................................................83

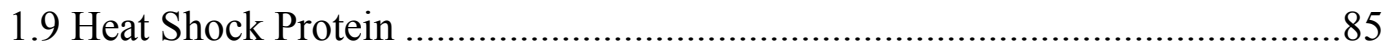

1.9.1 Molecular Chaperones and Tau Protein.......................................87

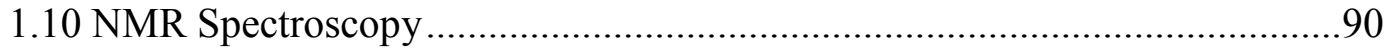

1.10.1 NMR Chemical Shifts.........................................................92

1.10.2 Heteronuclear Single Quantum Coherence................................93

1.10.3 Chemical Shift Mapping of Binding Sites ................................97

1.10.4 Site-Directed Spin Labeling of Proteins .....................................99

1.10.5 Equilibrium Denaturation .....................................................102

1.10.6 Diffusion Ordered Spectroscopy...............................................104

1.11 Complementary Biophysical Techniques .................................................106

1.11.1 Dynamic Light Scattering .....................................................106

1.11.2 Fourier Transform Infrared and Circular Dichroism ...............106

1.11.3 Small Angle X-ray Scattering ..................................................109 


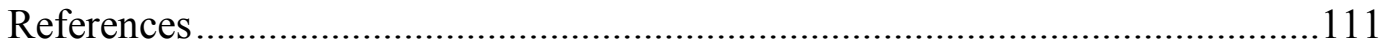

2. Inhibition of Tau Filament Formation by Conformational Modulation .....135

3. Mechanistic Basis of Phenothiazine-driven Inhibition of Tau Aggregation180

4. Imbalance of Hsp70 Family Variants Fosters Tau Accumulation...............207

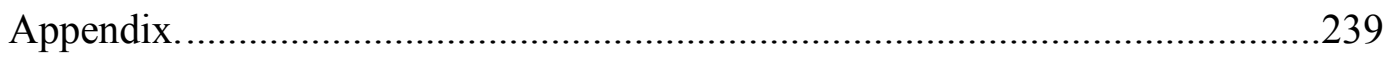

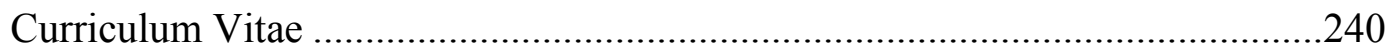




\section{List of Tables}

Table 1: Classification of Neurodegenerative Diseases. .............................27

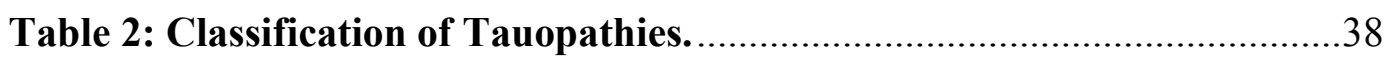

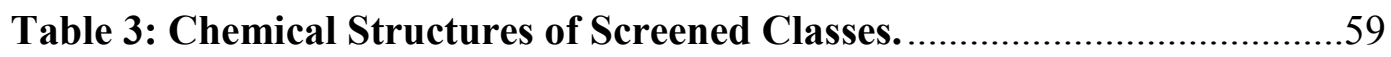

Table 3: Chemical Structures of Screened Classes. (Continue) ...................60

Table 4: Common NMR Parameters. ......................................................... 90 


\section{List of Figures}

Figure 1: The Energy Landscape of Protein Folding and Aggregation..........23

Figure 2: The Cascade of Protein Folding and Aggregation. ........................24

Figure 3: Pathological Hallmarks of Neurodegenerative Diseases................27

Figure 4: Classification of Intrinsically Disordered Proteins. ......................30

Figure 5: MAPT Gene and the Six Human Tau Isoforms...........................33

Figure 6: Primary Sequence of the Human Tau Protein hTau40.................34

Figure 7: Structural Polymorphism of Tau Protein. ..................................36

Figure 8: Neuronal Damage during Progression of Alzheimer's Disease. .....40

Figure 9: Hierarchical Assembly of Cross- $\beta$ Amyloid Fiber. ........................44

Figure 10: Proposed Cascade for the Pathological Aggregation of Tau........47

Figure 11: Tau-Based Therapeutic Strategies....................................... 49

Figure 12: The Self-stacking of PcTS as Detected by NMR Spectroscopy.....73

Figure 13: Reduction-Oxidation Interplay of Phenothiazines......................78

Figure 14: Conversion of Azure B to Quinoneimine. ...................................81

Figure 15: The Hierarchy of Heat Shock Proteins. ....................................87

Figure 16: Pulse Sequences for Basic and Constant-time HSQC...................94

Figure 17: HSQC Spectra of Folded and Disordered Proteins.....................96

Figure 18: Mapping of Chemical Shifts and Linewidths Changes................98

Figure 19: Site-directed Spin Labeling for PRE Measurements..................101

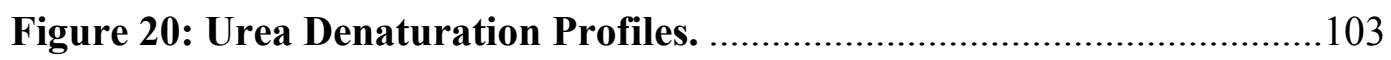

Figure 21: Equilibrium Denaturation of PcTS-stabilized Oligomers. ..........103

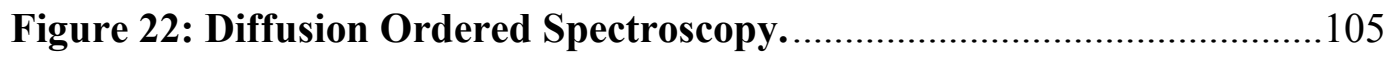

Figure 23: FTIR and CD Vibrational Spectroscopies. .............................108 
Figure 24: Small Angle X-ray Scattering and Geometric Structures.

.110 


\section{Abbreviations}

$\boldsymbol{\alpha} \quad$ Alpha Synuclein

A $\beta \quad$ Amyloid Beta

AD Alzheimer's Disease

AGD Argyrophilic Grain Disease

AFM Atomic Force Microscopy

ALS Amyotrophic Lateral Sclerosis

APP Amyloid Precursor Protein

ATP Adenosine triphosphate

ATPZ Aminothienopyridazine

BBB Blood Brain Barrier

BSE Bovine Spongiform encephalopathy

CaM Calmodulin

CaMKII Calcium/calmodulin dependent protein kinase II

cAMP Cyclic adenosine monophosphate

CBD Corticol-Basal Degeneration

CD Circular Dichroism

Cdk5 Cyclin-dependent kinase 5

CJD Creutzfeldt-Jakob disease

CWD Chronic Wasting Disease

DP Dementia Pugilistica

DS Down's Syndrome

EGCG (-) Epigallocatechin-3-gallate

EM Electron Microscopy

EPR Electron Paramagnetic Resonance

ESI-MS Electron Spray Ionization-Mass spectrometry 


\begin{tabular}{|c|c|}
\hline FBDD & Fragment-based drug design \\
\hline FRET & Fluorescence resonance energy transfer \\
\hline FTDP-17 & Fronto-Temporal dementia with Parkinsonism linked to chromosome 17 \\
\hline FTIR & Fourier Transform Infrared \\
\hline GSK3及 & Glycogen synthase kinase $3 \beta$ \\
\hline HCS & High content screening \\
\hline HD & Huntington's Disease \\
\hline Hsc & Heat shock cognate \\
\hline Hsp & Heat shock protein \\
\hline HTS & High throughput screening \\
\hline IDP & Intrinsically disordered protein \\
\hline INEPT & Insensitive nuclei enhancement through polarization transfer \\
\hline LDH & Lactate dehydrogenase \\
\hline LMB & Leuco Methylene Blue \\
\hline МАPK & Mitogen-activated protein kinase \\
\hline MAP2 & Microtubule-associated protein 2 \\
\hline MAPT & Microtubule-associated protein Tau \\
\hline MARK & Microtubule-affinity regulating kinase \\
\hline MAS & Magic Angle Spinning \\
\hline MB & Methylene Blue \\
\hline MBP & Maltose Binding Protein \\
\hline MT & Microtubule \\
\hline NADPH & Nicotinamide adenine dinucleotide phosphate \\
\hline NBD & Nucleotide Binding Domain \\
\hline ND & Neurodegenerative Disease \\
\hline NFT & Neurofibrillary tangle \\
\hline NMR & Nuclear magnetic resonance \\
\hline Pc & Phthalocyanine \\
\hline
\end{tabular}




\begin{tabular}{ll} 
PcTS & Phthalocyanine Tetrasulfonate \\
PET & Positron emission tomography \\
PHF & Paired helical filament \\
PKA & cAMP-dependent protein kinase A \\
PKC & cAMP-dependent protein kinase C \\
PD & Parkinson's Disease \\
PiD & Pick's Disease \\
PTH & Phenylthiazolhydrazide \\
PP & Protein phosphatase \\
PRE & Paramagnetic Relaxation Enhancement \\
PrP-res & Protease-resistant Prion \\
PrP-sen & Protease-sensitive Prion \\
PSP & Progressive Supranuclear Palsy \\
SAR & Structure-activity relationship \\
SAXS & Small Angle X-ray Scattering \\
SBD & Substrate Binding Domain \\
SOD1 & Superoxide Dismutase-1 \\
STD & Saturation transfer difference \\
SUMO1 & Small ubiquitin-like modifier protein 1 \\
TESK1 & Testis-specific protein kinase 1 \\
ThS & Thioflavin S \\
TSE & Transmissible Spongiform Encephalopathies \\
\hline
\end{tabular}




\section{Introduction}

\section{Introduction}

Proteins are versatile macromolecules that constitute a network of structurally complex systems with crucial biological relevance in life. Through the course of evolution, mammalian cells have adapted machineries that synthesize linear chains of several amino acids and ensure the conformational integrity of folding polypeptides to their native state with defined three-dimensional structures. Typically, these cells regulate the expression of more than 10,000 different protein species, which makes them prone to acute and severe malfunctions. Yet, this highlights the fundamental role and medical relevance of proteins in biology. The remarkable features of protein folding define its functionality by the ability of retaining its proper conformation. However, many proteins or protein domains are functional despite their partially or fully unstructured conformations. Nevertheless, the outstanding characteristic of such disordered or misfolded proteins decodes in the generation of proteinaceous aggregates of highly defined structures with abundant cross- $\beta$ structural motifs, termed amyloid. These amyloidogenic proteins are the basis of

pathological processes that embraces numerous diseases, most important being Neurodegeneration. 


\subsection{Protein Folding, Misfolding, and Aggregation}

In nature, a protein folds to acquire a biologically-active native state defined by a precise three-dimensional structure. This complicated cascade is achieved either spontaneously or with enzymatic assistance from molecular chaperones. ${ }^{1,2}$ Yet, deeper understanding of this intricate network remains partially undisclosed. Nevertheless, intensive pioneering studies have investigated the nature of folding mechanisms and implicated transitional states with their kinetic, thermodynamic and structural information. ${ }^{3-5}$ Based on configurational entropy, the folding energy landscape of a protein has a funnel-shaped profile that represents the pathway by which folding or aggregation follows (Figure 1). An unfolded state of various conformations (1) adapts specific changes, and through intramolecular contacts, it populates folding intermediates (2) that further proceed to the de novo native state (3). Since the energy landscape surface and its ruggedness are unique to the primary sequence of the protein ${ }^{6}$ potential domain interactions increase the propensity of misfolded conformations. If failed to reorganize, this partially folded state (4) is prone to aggregation since it favors a major kinetic trap during folding pathways. Driven by intermolecular contacts, the exposed hydrophobic residues then induce the association of protein molecules into oligomers (5) that further assemble into amorphous aggregates (6) or trigger the formation of highly ordered amyloid fibrils (7). Whether progressing through folding or aggregation, it is astonishing how the high degree of disorder of an unfolded state is reduced by selecting the more favorable enthalpy and the lowest entropy so the structure becomes more ordered, being native or fibrillar. 


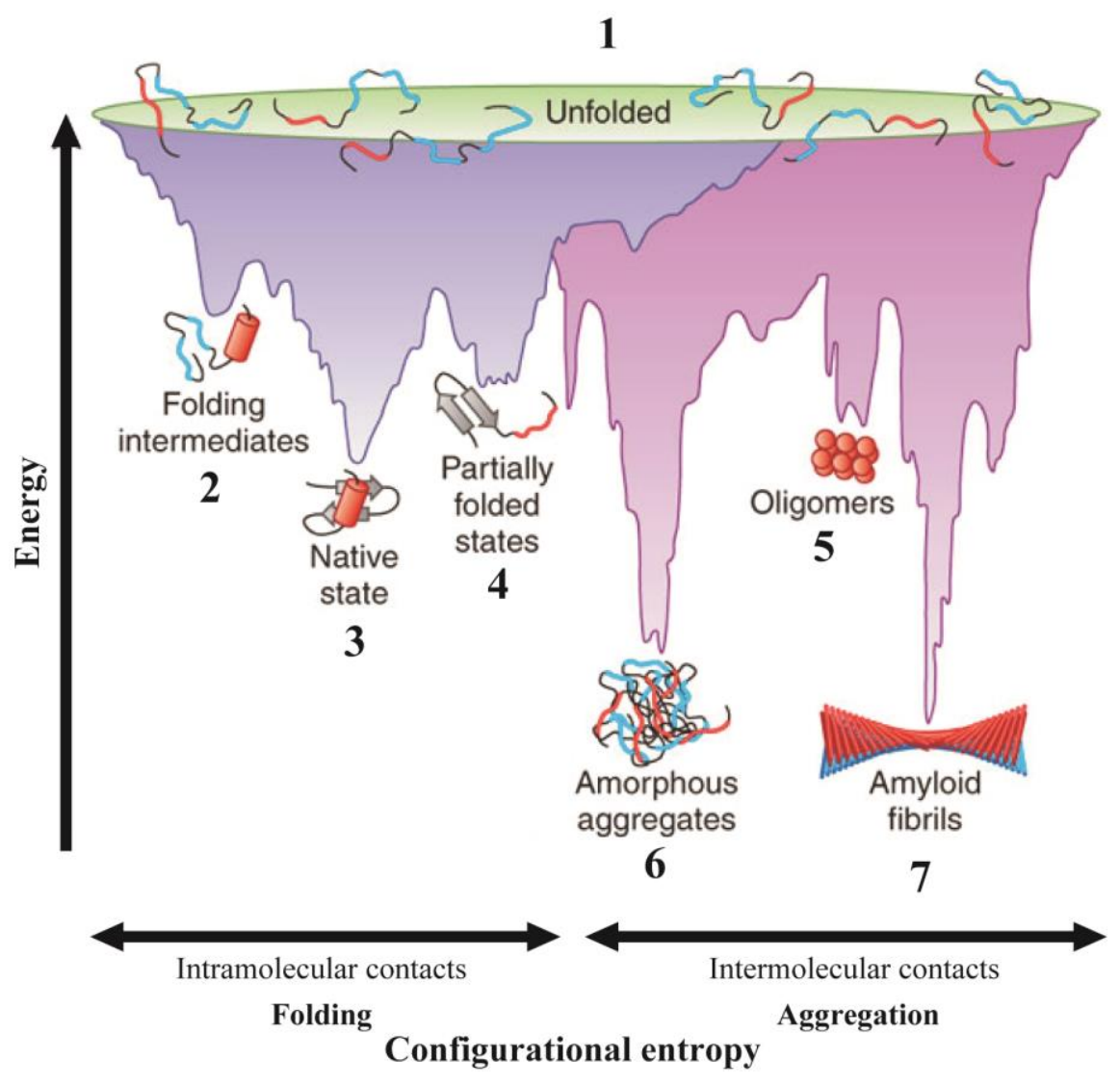

Figure 1: The Energy Landscape of Protein Folding and Aggregation.

a) The multitudinal conformations of an unfolded protein (1) populate folding intermediates (2) while funneling through the energy landscape towards a single de novo native state (3). Partially folded states (4) are kinetically trapped as oligomers (5), which further deposit as amorphous (6) and amyloid fibrillar (7) aggregates. Adapted from ${ }^{6,7}$.

Protein folding and unfolding are ultimate biological events that regulate cellular trafficking, secretions and immune response. Aberrations in these mechanisms impede cell regulations and initiate diseases that are often accompanied by the conversion of misfolded intermediates to aggregates. Despite the diversity in the pathogenesis of these diseases, the aggregation of a functional soluble protein into insoluble amyloid fibers with cross- $\beta$ 
structural motifs arises by common mechanisms and involves several species (Figure 2). ${ }^{4,8}$ A native state is highly sensitive to destabilizing conditions (low $\mathrm{pH}$, high temperature, mutation, presence of denaturant), thereby increasing the population of the misfolded state. ${ }^{9}$ If molecular chaperones and ubiquitin-proteasome machineries fail to refold or clear the partly folded species back to the normal state or to amorphous aggregates, ${ }^{10-12}$ then nucleation of ordered oligomers becomes kinetically favored. With an increased propensity to cross- $\beta$ structures, the amyloidogenic precursors have the potential to progressively assemble through membrane embedded pores and lateral-aggregating protofibrils. This stream of aggregates is finally deposited in forms of mature amyloid fibers.

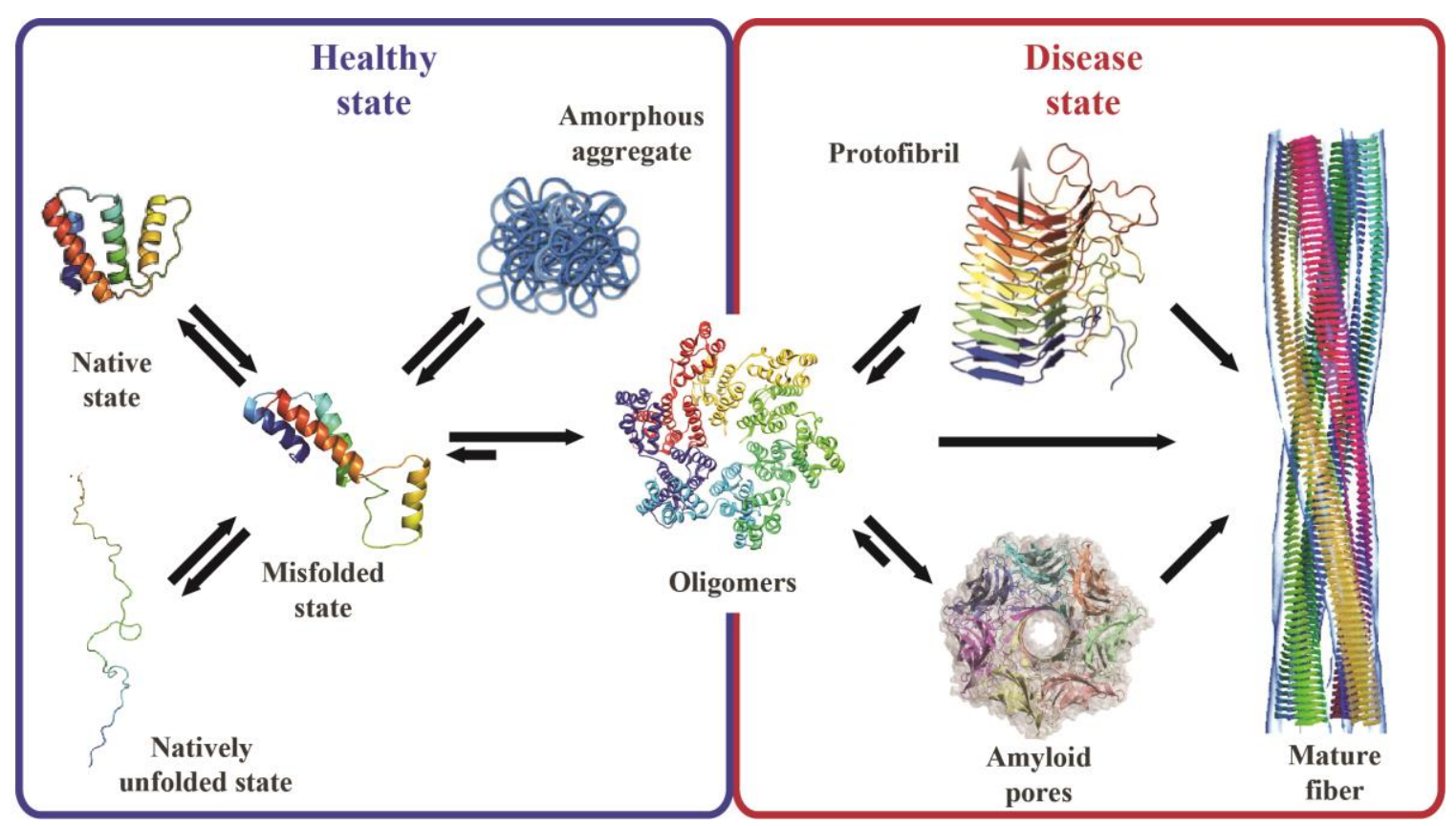

Figure 2: The Cascade of Protein Folding and Aggregation.

Destabilizing a healthy native state increases the population of the misfolded state. Refolding or degrading pathways along with external factors aim to re-establish proper folding, formation of amorphous aggregates or complete unfolding. The failure of these defensive mechanisms triggers the nucleation of ordered oligomers that assemble into amyloid pores, protofibrils and mature fibers. The whole assembly process provokes cell impairment and gained toxicity that defines this disease state. Modified from ${ }^{4,6}$. 


\subsection{Neurodegenerative Diseases}

Dementia is a wide term for collective symptoms that include:

1. severe deterioration of concentration and judgment,

2. loss of memory and intellectual capacity,

3. subsequent damage of brain neurons,

4. progressive motor impairment.

Until the end of the nineteenth century, diagnosis remained within a broad clinical concept. In 1907, Alois Alzheimer and Oskar Fischer associated the presence of abundant aggregates of neuritic amyloid plaques and neurofibrillary tangles (NFTs) in the cerebral cortex to dementia that was pathologically termed Alzheimer's Disease (AD). ${ }^{13-15}$ In 1911, Alzheimer observed specific inclusions related to frontotemporal dementia, ${ }^{16}$ which was later referred to as Pick's Disease (PiD) after Arnold Pick. A year later, Fritz Heinrich Lewy associated certain inclusions as features in Parkinson's Disease (PD) and identified them as Lewy bodies. ${ }^{17,18}$ Over the years that followed, the notion of Neurodegenerative Diseases (ND) experienced substantial progress in brain study with attempts to uncover molecular and pathological bases. Soon after, this area of research has moved from descriptive phenomenology to mechanistic analysis.

Clinical diagnosis of different neurodegenerative brain tissues revealed that the pathological markers (Figure 3) consisted primarily of aggregates related to specific proteins that can be cytoplasmic, nuclear or extracellular. ${ }^{19}$ These aggregates and their corresponding proteins were identified respectively as amyloid plaques of amyloid-Beta (A $\beta),{ }^{20}$ NFTs of Tau, ${ }^{21}$ Lewy bodies of alpha-synuclein $(\alpha \mathrm{S}),{ }^{22}$ neuronal inclusions of huntingtin, ${ }^{23}$ prion plaques of protease-resistant prion (PrP-res), ${ }^{24}$ and Bonina bodies of Superoxide dismutase-1 (SOD1). ${ }^{25}$ Today, it is widely recognized that the generation of 
protein aggregates with rich $\beta$-sheet conformation and inclusion body formation is a common pathological process in numerous neurodegenerative diseases. ${ }^{26}$ Nevertheless, each disorder is associated to its own set of pathological features (Table 1). The most common neurodegenerative diseases are classified based on their clinical symptoms, the affected regions of the brain, their pathological hallmarks and the involved proteins. ${ }^{19,27,28}$

$\mathrm{AD}$ remains the most widespread progressive dementia syndrome featuring neuronal degeneration along with the presence of extracellular A $\beta$ and intracellular NFTs. Importantly, the cytoplasmic Lewy body, a pathological hallmark of PD, is linked to the degeneration of dopaminergic neurons and consequently to movement disorders, postural and autonomic instability. Impaired muscle coordination and motor activity are common symptoms of the inherited neurological Huntington's disease (HD). The neuronal inclusions are nuclear deposits of Huntingtin protein with abnormal long polyglutamine stretches at the N-terminal region. Transmissible Spongiform encephalopathies (TSE) are diseases showing extracellular prion plaques as pathological markers. These syndromes are spread among different species; mostly featuring dementia, psychiatric breakdown and cortical spongiform degeneration. Amyotrophic Lateral Sclerosis (ALS) is a fatal disease characterized by the degeneration of motor neurons and atrophy. The major hallmarks of this disease are cytoplasmic Bonina bodies composed of SOD1. 


\subsection{Neurodegenerative Diseases}

\section{Alzheimer's Disease \\ Parkinson's Disease}

(AD)

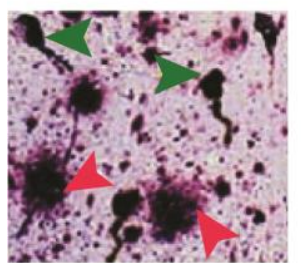

Amyloid plaques

Neurofibrillary tangles
(PD)

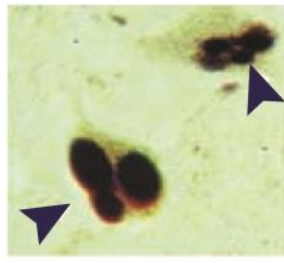

Lewy bodies
Huntington's

Disease

(HD)

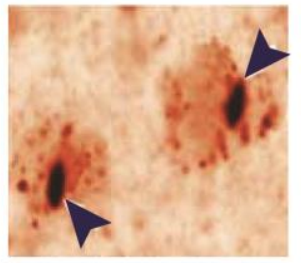

Neuronal inclusions
Transmissible

Spongiform

Encephalopathies

(TSE)

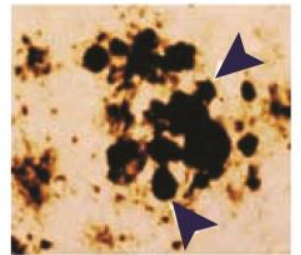

Prion Plaques
Amyotrophic

Lateral

Sclerosis

(ALS)

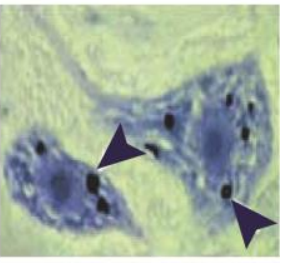

Bonina bodies

Figure 3: Pathological Hallmarks of Neurodegenerative Diseases.

The neuropathological lesions of common neurodegenerative diseases: AD's extracellular plaques and intracellular NFTs; the cytoplasmic Lewy bodies of PD, neuronal inclusions of HD, TSE's extracellular prion plaques, and ALS cytoplasmic Bonina bodies. Adapted from ${ }^{19}$.

Extensive studies hypothesize that these insoluble deposits do not induce neurotoxicity but rather the soluble oligomeric intermediates formed during the aggregation process are the most toxic species. ${ }^{26,29,30}$ This strengthens the notion that diverse ND pathogeneses indeed arise from common mechanisms.

\section{Table 1: Classification of Neurodegenerative Diseases.}

NDs are classified based on their clinical symptoms, the affected regions of the brain, proteins involved and pathological hallmarks. Adapted from ${ }^{19,27,28}$.

\begin{tabular}{|c|c|c|c|c|c|}
\hline Disease & Clinical Symptoms & Affected Regions & Protein Involved & Pathology markers & Location \\
\hline $\begin{array}{l}\text { Alzheimer's } \\
\text { Disease (AD) }\end{array}$ & $\begin{array}{l}\text { Progressive dementia } \\
\text { Cognitive impairments }\end{array}$ & $\begin{array}{l}\text { Hippocampus, cortex, } \\
\text { forebrain, brain stem }\end{array}$ & $\begin{array}{l}\text { Amyloid- } \beta \text {, } \\
\text { Tau }\end{array}$ & $\begin{array}{l}\text { Amyloid plaques, } \\
\text { neurofibrillary tangles }\end{array}$ & $\begin{array}{l}\text { Extracellular, } \\
\text { cytoplasmic }\end{array}$ \\
\hline $\begin{array}{l}\text { Parkinson's } \\
\text { Disease (PD) }\end{array}$ & Movement Disorder & $\begin{array}{l}\text { Substantia nigra, dorsal } \\
\text { motor, hypothalamus }\end{array}$ & $\alpha$-Synuclein & Lewy bodies & Cytoplasmic \\
\hline $\begin{array}{l}\text { Huntington's } \\
\text { Disease (HD) }\end{array}$ & $\begin{array}{l}\text { Dementia, reduced } \\
\text { motor activity }\end{array}$ & $\begin{array}{l}\text { Striatum, cortex, } \\
\text { basal ganglia }\end{array}$ & Huntingtin & Neuronal inclusions & Nuclear \\
\hline $\begin{array}{l}\text { Transmissible } \\
\text { Spongiform enc- } \\
\text { ephalopathies (TSE) }\end{array}$ & $\begin{array}{l}\text { Dementia, ataxia, } \\
\text { psychiatric breakdown }\end{array}$ & $\begin{array}{l}\text { Thalamus, cortex, } \\
\text { cerebellum }\end{array}$ & $\begin{array}{l}\text { Protease-resistant } \\
\text { prion protein }\end{array}$ & $\begin{array}{l}\text { Prion Plaques } \\
\text { Spongiform degeneration }\end{array}$ & Extracellular \\
\hline $\begin{array}{l}\text { Amyotrophic } \\
\text { Lateral } \\
\text { Sclerosis (ALS) }\end{array}$ & Movement disorder & $\begin{array}{l}\text { Spinal motor neurons, } \\
\text { motor cortex }\end{array}$ & $\begin{array}{l}\text { Superoxide } \\
\text { dismutase-1 }\end{array}$ & $\begin{array}{l}\text { Bonina bodies, } \\
\text { axonal spheroids }\end{array}$ & Cytoplasmic \\
\hline
\end{tabular}




\subsection{Intrinsically Disordered Proteins}

The discovery of intrinsically discorded proteins (IDPs) was accompanied by an evolutionary paradigm in the field of structural biology. Proteins that fail to adopt a defined three dimensional structure under physiological conditions are termed IDPs and are represented as dynamic conformational ensembles with the characteristic of a weakly funneled energy landscape. ${ }^{31}$ Nevertheless, a significant fraction of partially and completely disordered proteins are functionally active in eukaryotic cells ${ }^{32}$ and are involved in cellular processes such as signaling, transcription control, replication, and molecular recognition. ${ }^{33-35}$ IDPs are also prone to lose their physiological function and exhibit pathological features that appear in cancer, neurodegenerative diseases, cardiovascular diseases and others. ${ }^{36}$

\subsubsection{Structural Characteristics}

The prediction of the three-dimensional structures of globular proteins from their primary sequence is challenging, however, the analysis of its composition and relative occurrences of the amino acids has been established to identify intrinsically disordered domains. ${ }^{37}$ The sequence signature of an intrinsic disorder is reflected by a low content of bulky hydrophobic order-promoting residues (Asparagine, Cysteine, Histidine, Isoleucine, Leucine, Phenylalanine, Threonine, Tryptophan, Tyrosine, and Valine) and the abundant presence of polar and charged disorder-promoting (Arginine, Aspartic acid, Glutamic acid, Glutamine, Lysine, Methionine, Proline, and Serine) and neutral residues (Alanine and Glycine).$^{38-40}$ Despite their highly unfolded states, IDPs have the tendency to form low content of local secondary structures. Due the lack of specific tertiary structure, IDPs are 


\subsection{Intrinsically Disordered Proteins}

commonly represented by ensembles of conformations with defined $\phi$ and $\psi$ backbone angles. ${ }^{37}$ However, IDPs are clearly distinguished from both, folded and completely unfolded proteins. IDPs do not behave like the denaturated states of folded proteins in terms of true random coils. Even at extreme $\mathrm{pH}$, temperature and pressure, the propensity for local structures and hydrophobic clusters is to some extent retained. ${ }^{41}$

\subsubsection{Biological Function}

Accumulating evidence from genomic analysis of disordered proteins demonstrated the vital biological activity of IDPs despite the lack of a well-defined structure. ${ }^{33,42-44}$ These proteins maintain various biological processes and specific signaling interactions that are controlled by numerous posttranslational modifications and alternative splicing. This comprises transcriptional regulation of DNA binding, mRNA splicing, biogenesis of chromatin, and intricate organization of the cytoskeleton. ${ }^{45}$ Some IDPs undergo a disorderto-order transition upon binding to one or more partner proteins ${ }^{46,47}$ while others carry normal functions despite their disordered state. Based on functionality and binding modes, IDPs have been classified into different categories (Figure 4). ${ }^{33}$ Some IDPs that are functioning irrespective of any binding partner are termed Entropic chains. These include disordered regions or full proteins that incorporate entropic springs and spacers between two adjacent domains or flexible linkers that facilitate conformational modulations. Other IDPs function upon molecular recognition of their partners via transient or permanent binding. For instance, molecular chaperones exhibit transient activities through their disordered regions to assist RNA and protein folding. Alternatively, an IDP might display sites to accommodate the targeted protein for further posttranslational modifications. On 


\subsection{Intrinsically Disordered Proteins}

the other hand, IDPs with permanent bindings fall in three categories. Effectors inhibit or activate their partner proteins, assemblers favor formation of large complexes while scavengers target smaller ligands.

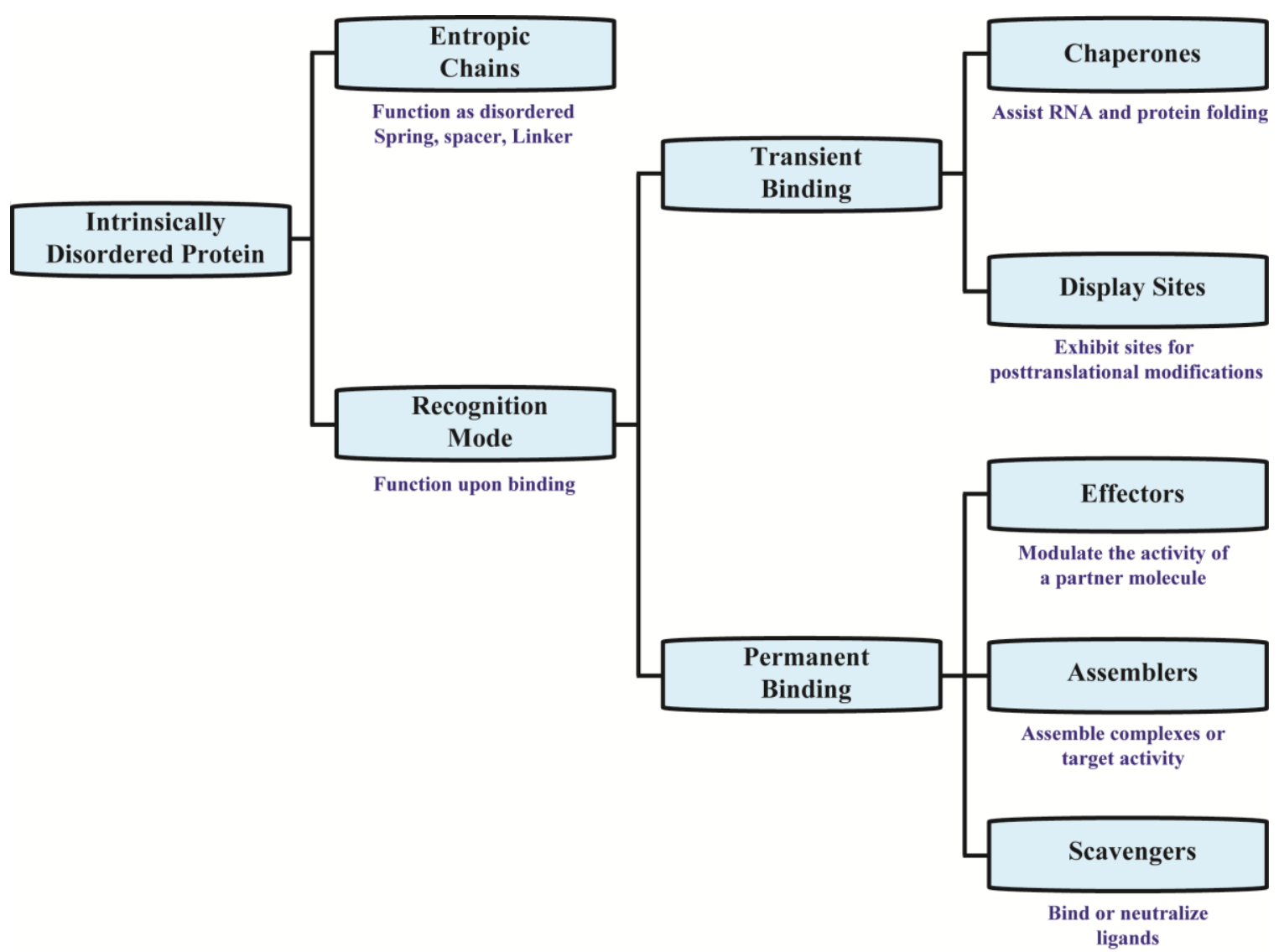

Figure 4: Classification of Intrinsically Disordered Proteins.

Based on their function, IDPs are classified into major categories. Entropic chains function irrespective of partner binding. Other IDPs bind their partners via transient (chaperones, displayed sites) or permanent recognition modes (effectors, assemblers, scavengers). Adapted from ${ }^{33}$.

Several proteins associated with human neurodegenerative diseases are intrinsically disordered. As mentioned before, each of the proteins $\mathrm{A} \beta$, Tau, $\alpha \mathrm{S}$, huntingtin, PrP-res and SOD1 exists or coexists in the ND hallmarks amyloid plaques, NFTs, lewy bodies, 


\subsection{Intrinsically Disordered Proteins}

neuronal inclusions, prion plaques and bonina bodies, respectively. These proteins either contain intrinsically disordered regions or themselves are IDPs. The generation of these protein aggregates from a natively unfolded state to a rich $\beta$-sheet conformation proved the interconnection between intrinsic disorder and human neurodegenerative diseases.

\subsection{Tau Protein}

The isolation of a heat stable protein purified from porcine brain, identified as Tau, was an outstanding discovery in the field of neurobiology. ${ }^{48}$ Linked to the dimerization of $\alpha$ and $\beta$ tubulin, Tau was the first to be reported as a microtubule-associated protein soon after its sequence was determined from murine cDNA. ${ }^{49}$ Tau protein has gained significant prominence as it is associated to a physiological function in the regulation of MT stability and a pathological hallmark in AD and other NDs.

\subsubsection{Localization, Function and Modifications}

MAPT is a single gene located on chromosome $17 \mathrm{q} 21^{50}$ and contains 16 exons, 11 of which are engaged for encoding Tau protein in the human brain (Figure 5). The alternative mRNA splicing of exons 2, 3, and 10 generates six different Tau isoforms ranging from 352 to 441 amino acids. These isoforms are distinguished by the absence or presence of one (I1) or two (I2) inserts at the N-terminal domain and a repeat region (R2) within a repeating-motifs domain. ${ }^{51}$ The constitutive splicing of the remaining exons encode for the motifs that are present among all isoforms (exons 1, 4, 5, and 7 for the Nterminus and Proline-rich region P1; exons 9, 11 and 12 for P2 and repeat motifs R1, R3 and R4; and exon 13 for $\mathrm{R}^{\prime}$ and the $\mathrm{C}$-terminus). The 5 additional exons are not involved 
in Tau expression. Exons 0 and 14 are both non-coding, exon $4 \mathrm{a}$ is associated only to the peripheral nervous system, whereas the transcription of exons 6 and 8 is prohibited in the human brain. ${ }^{52}$

The composition of the six Tau isoforms have been categorized into two large domains: the projection domain and the microtubule-binding domain (Figure 5). The domain organization of Tau is based on the primary sequence of the longest human isoform, hTau40 (Figure 6). The projection domain combines two regions, a highly negatively charged region incorporating the abundant number of acidic residues of the Nterminus and the inserts $(\mathrm{I} 1, \mathrm{I} 2)$, followed by the first Proline-rich region (P1). As the name refers, the second domain accommodates the regions that have been identified for their binding to microtubules. These four motifs, termed Pseudo-repeats (R1, R2, R3, R4), are imperfect copies of a sequence of 18 amino acids scattered between inter repeating sequence of 13 or 14 amino acids. Together with the second Proline-rich region and the less conserved pseudo-repeat $\left(\mathrm{R}^{\prime}\right)$, all these three regions define the interface upon which Tau binds to microtubules through a highly conserved tubulin-binding motif. ${ }^{49}$ The postnatal expression of exon 10 distinguishes the isoforms containing 3-repeats (3R) from 4-repeats (4R) based on the existence of R2. ${ }^{51}$ During development, the various isoforms are differentially expressed, however, $3 \mathrm{R}$ and $4 \mathrm{R}$ isoforms are equally expressed in cerebral cortex of healthy adults. ${ }^{53}$ Notably, the R1 and R2 provoke the highest capacity to bind tubulin $^{54}$ and this is in line with the fact that $4 \mathrm{R}$ adult Tau isoforms interact with microtubules more efficient. ${ }^{55,56}$ On the other hand, the projection domain interacts with cytoskeleton and plasma membrane proteins. ${ }^{57,58}$ Moreover, specific motifs were highlighted upon its binding to cations, heparin (KKXK), and proteins containing the SH3 domain (PXXP). ${ }^{59}$ 


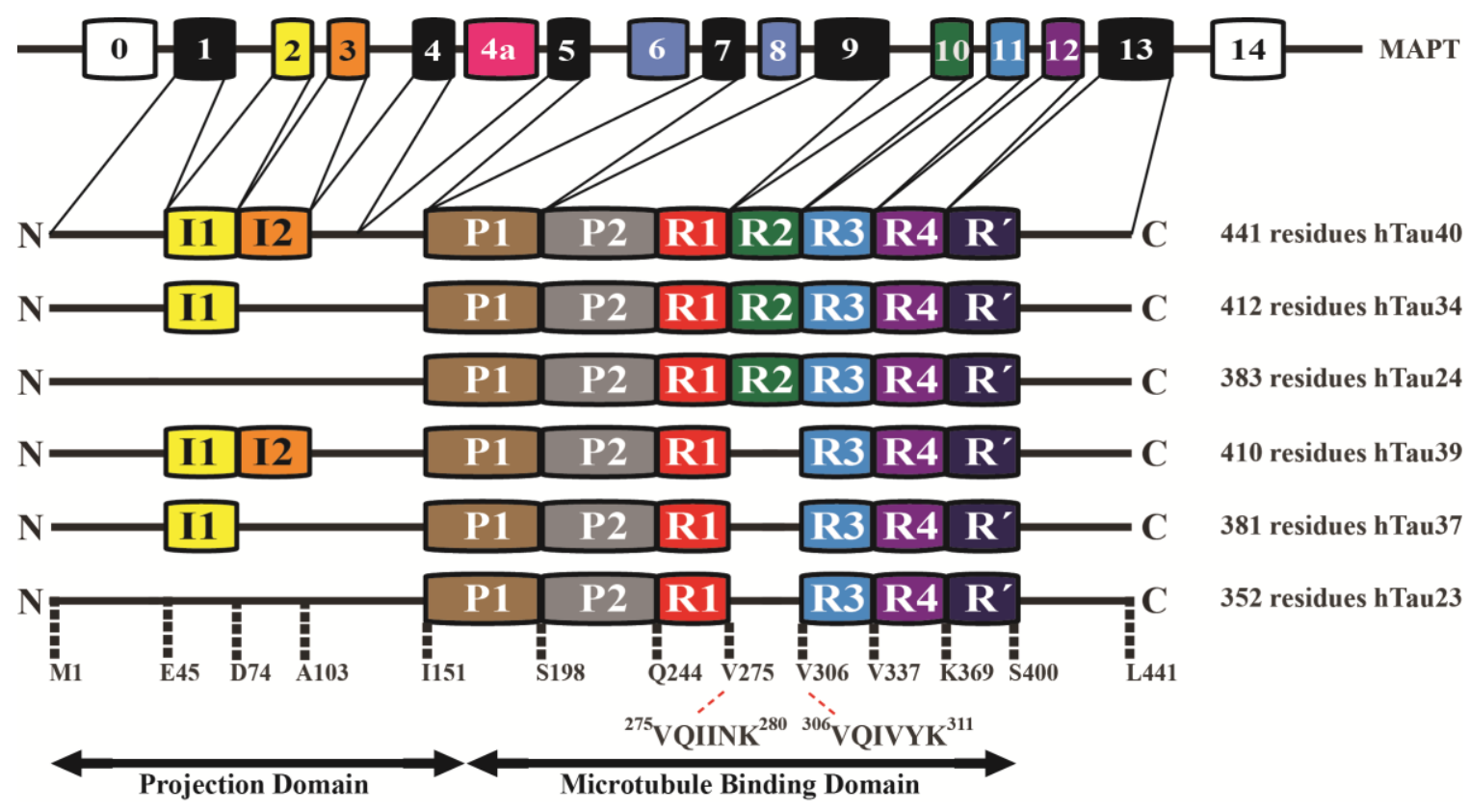

Figure 5: MAPT Gene and the Six Human Tau Isoforms.

MAPT consists of 16 exons in which the alternative mRNA splicing of E2, E3, and E10 generates the six human isoforms. The highlighted regions correspond to the domain organization present among the isoforms. ( $\mathrm{N}$ or $\mathrm{C}=\mathrm{N}$ or $\mathrm{C}$ terminus, $\mathrm{I}=$ insert, $\mathrm{P}=$ proline-rich regions, $\mathrm{R}=$ pseudo-repeat). Modified from ${ }^{52,60}$.

Sequencing studies showed high conservation of the microtubule-binding repeats across various species including mouse, rat, cow, monkey, goat and chicken. ${ }^{49,61}$ Tau is mainly expressed and located in the neuronal cells where it conceives its assembly with microtubules but also potentially associates with the plasma membrane ${ }^{62,63}$ after its phosphorylation in the cytosol. ${ }^{64}$ Tau expression has been reported to be higher in the grey matter of the neocortex than in the white matter. ${ }^{65}$ Importantly, non-neuronal Tau expression has been observed in muscle, liver, and kidney; ${ }^{66}$ as it has been confirmed in breast, prostate, gastric and pancreatic cancer lines. ${ }^{67-71}$ To date, the function of nonneuronal Tau remains unclear. 


\begin{tabular}{|c|c|c|c|c|}
\hline Positive & Negative & Hydrophobic & Hydrophilic & \\
\hline $\begin{array}{ll}10 \\
\text { MAEPRQE }\end{array}$ & $\begin{array}{ll}20 \\
\text { MEDHAGT }\end{array}$ & $\begin{array}{r}30 \\
\text { GDRKDQGG TI }\end{array}$ & $\begin{array}{r}40 \\
\text { MHQDQEGDTD }\end{array}$ & $\begin{array}{r}50 \\
\text { AGLKESPLQT }\end{array}$ \\
\hline $\begin{array}{r}60 \\
\text { PTEDGSEEPG }\end{array}$ & $\begin{array}{r}70 \\
\text { SETSDAKSTP }\end{array}$ & $\begin{array}{r}80 \\
\text { TAEDVTAPLV }\end{array}$ & $\begin{array}{r}90 \\
\text { DEGAPGKOAA }\end{array}$ & $\begin{array}{r}100 \\
\text { AQPHTEIPEG }\end{array}$ \\
\hline $\begin{array}{r}110 \\
\text { TTAEEAGIGD }\end{array}$ & $\begin{array}{r}120 \\
\text { TPSLEDEAAG }\end{array}$ & $\begin{array}{r}130 \\
\text { HVTQARMVSK }\end{array}$ & $\begin{array}{r}140 \\
\text { SKDGTGSDDK }\end{array}$ & $\begin{array}{r}150 \\
\text { KAKGADGKTK }\end{array}$ \\
\hline $\begin{array}{r}160 \\
\text { IATPRGAAPP }\end{array}$ & $\begin{array}{r}170 \\
\text { GQKGQANATR }\end{array}$ & $\begin{array}{r}180 \\
\text { IPAKT PPAPK }\end{array}$ & $\begin{array}{r}190 \\
\text { TPPSSGEPPK }\end{array}$ & $\begin{array}{r}200 \\
\text { SGDRSG SSP }\end{array}$ \\
\hline $\begin{array}{r}210 \\
\text { GSPGTPGSRS }\end{array}$ & $\begin{array}{r}220 \\
\text { RTPSLPTPPT }\end{array}$ & $\begin{array}{r}230 \\
\text { REPKKVAVVR }\end{array}$ & $\begin{array}{r}240 \\
\text { TPPKSPSSAK }\end{array}$ & $\begin{array}{r}250 \\
\text { SRLQTAPVPM }\end{array}$ \\
\hline $\begin{array}{r}260 \\
\text { PDLKNVKSKI }\end{array}$ & $\begin{array}{r}270 \\
\text { GSTENLKHQP }\end{array}$ & $\begin{array}{r}280 \\
\text { GGGKVQIINK }\end{array}$ & $\begin{array}{r}290 \\
\text { KLDLSNVQSK }\end{array}$ & $\begin{array}{r}300 \\
\text { CGSKDNIKHV }\end{array}$ \\
\hline $\begin{array}{r}310 \\
\text { PGGGSVQIV }\end{array}$ & $\begin{array}{r}320 \\
\text { KPVDISKVTS }\end{array}$ & $\begin{array}{r}330 \\
\text { KCGSLGNIHH }\end{array}$ & $\begin{array}{r}340 \\
\text { KPGGGQVEVK }\end{array}$ & $\begin{array}{r}350 \\
\text { SEKLD KDRV }\end{array}$ \\
\hline $\begin{array}{r}360 \\
\text { QSKIGSLDNI }\end{array}$ & $\begin{array}{r}370 \\
\text { THVPGGGNKK }\end{array}$ & $\begin{array}{r}380 \\
\text { IETHKLT RE }\end{array}$ & $\begin{array}{r}390 \\
\text { NAKAKTDHGA }\end{array}$ & $\begin{array}{r}400 \\
\text { EIV KSPVVS }\end{array}$ \\
\hline $\begin{array}{r}410 \\
\text { GDTSPRHLSN }\end{array}$ & $\begin{array}{r}420 \\
\text { VSSTGSIDMV }\end{array}$ & $\begin{array}{r}430 \\
\text { DSPQLATLAD }\end{array}$ & $\begin{array}{r}\mathbf{4 4 0} \\
\text { EVSASLAKQG }\end{array}$ & L \\
\hline
\end{tabular}

Figure 6: Primary Sequence of the Human Tau Protein hTau40.

The primary sequence of the longest Tau isoform contains two negatively charged inserts (I1, I2) two prolinerich regions $(\mathrm{P} 1, \mathrm{P} 2)$, four pseudo-repeat regions $(\mathrm{R} 1, \mathrm{R} 2, \mathrm{R} 3, \mathrm{R} 4)$ and one repeat-like region $\left(\mathrm{R}^{\prime}\right)$. The amino acids are color-coded based on their chemical properties.

Tau phosphorylation is the most significant posttranslational modification (Figure 7) with extensive studies focusing on serine/threonine $e^{72,73}$ and recently on tyrosine phosphorylation sites. ${ }^{74}$ Inside the cell, the distribution of Tau is influenced by phosphorylation. For instance, Tau with modified sites of the proline-rich region occurs in the somatodendritic compartment, but is translocated to the distal axonal region once these sites are dephosphorylated or as a consequence of additional phosphorylation at the Cterminus. ${ }^{54,74}$ The regulation of Tau phosphorylation is crucial to its affinity for microtubules especially during early developing neurons, with evidence implicating higher phosphorylation levels in fetal Tau than adult Tau. ${ }^{75}$ hTau40 accommodates 85 putative 
phosphorylation sites (45 serines, 35 threonines, 5 tyrosines) ${ }^{76}$ that are phosphorylated either by (a) proline-directed kinases (GSK3 $\beta$, cdk5, MAPK, p38) ${ }^{77-79}$ or by (b) nonproline directed kinases (PKA, PKC, MARK, CaMKII). ${ }^{80}$ The first group of kinases targets the 17 serine/proline (SP) and threonine/proline motifs (TP) whereas the other sites are phosphorylated by the kinases of the second group. Most importantly is the phosphorylation of the serine residues of the 4 KXGS motifs spread along the 4 repeat regions (S262, S293, S324, and S356). ${ }^{81}$ Additionally, S214 is upregulated during mitosis and is exclusively phosphorylated by PKA. ${ }^{82}$ On the other hand, certain residues such as T231 are termed prime epitopes meaning that their phosphorylation is only possible whenever the fourth succeeding residue is a phosphorylated serine or threonine, in this case $\mathrm{S} 235^{83}$

Like most amyloid forming proteins, recombinant Tau is natively unfolded with a high fraction of basic and hydrophilic amino acids that resist compact folding and result in low content of secondary structure (Figure 7). ${ }^{84}$ Additionally, Tau is resilient to heat and acid treatment. ${ }^{85}$ Both, flexibility and lack of an ordered structure impede the high resolution analysis of IDPs by X-ray crystallography, thus leaving NMR spectroscopy as the only descriptive technique to access the conformations and dynamics of natively unfolded proteins. Indeed, the complete backbone resonance NMR assignment of the fulllength human Tau protein was achieved ${ }^{86}$ in a divide and conquer strategy. ${ }^{56,87,88}$ Tau was found to be highly dynamic in solution with a distinct domain character and an intricate network of transient long-range contacts. ${ }^{86}$ Further analysis of Tau fragments incorporating only 3R (K19) and 4R (K18) repeat domains unveiled short stretches that (1) adopt highly populated turn conformations in the monomeric form $^{88}$, (2) fold into $\alpha$-helical 
conformations upon binding to lipid surfaces ${ }^{89}$ and (3) consist of $\beta$-strands in the course of filament formation. ${ }^{90,91}$
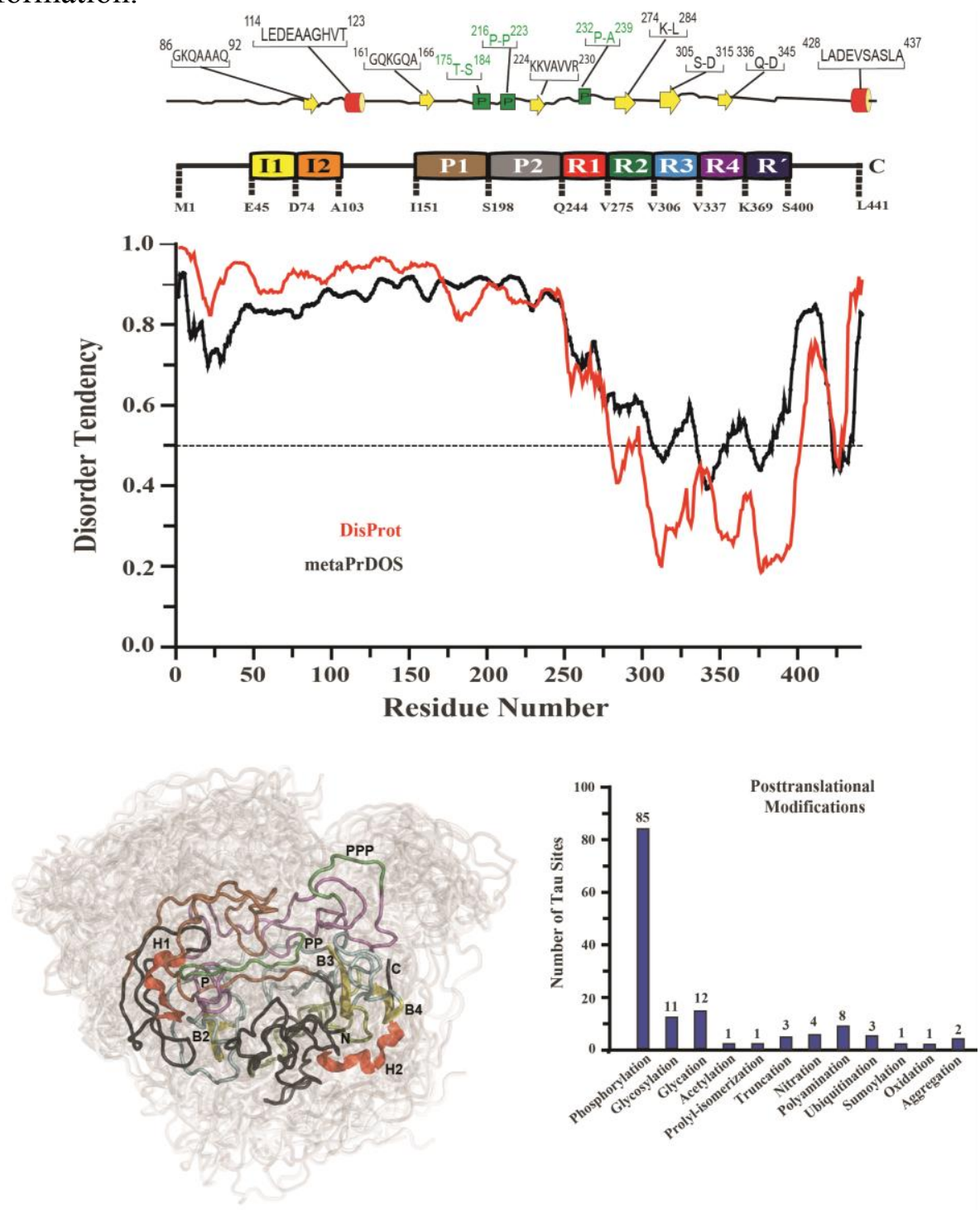

Figure 7: Structural Polymorphism of Tau Protein.

The prediction of disorder in the primary sequence of hTau40 is compared to experimental secondary structure propensities. The ensemble structural representation of hTau40 is color-coding the domain organization and overlaying 20 conformations. Tau undergoes numerous posttranslational modifications that involve a large number of sites as depicted in the graph. Disorder prediction was performed by the meta predictors DisProt ${ }^{92}$ and metaPrDOS. ${ }^{93}$ The transient secondary structures and ensembles were adapted from ${ }^{86}$. Posttranslational modifications were adapted from ${ }^{74}$. 
In eukaryotic cells, MTs constitute an indispensable component of the cytoskeleton with diverse functions ranging from regulation of cell shape and growth to intracellular machinery transport. MTs are highly dynamic cylindrical structures assembled by polymerized $\alpha$ - and $\beta$-tubulin heterodimers. The high expression of Tau protein in the neuronal axons is directly connected to its functions in machinery transport. ${ }^{94}$ The primarily role associated to Tau existence is its modulation to the stability and flexibility of axonal microtubules upon interaction with tubulin. Together with the microtubuleassociated protein 2 (MAP2), Tau shifts the reaction kinetics in favor of promoting MT assembly. Despite the fact that the structure of MT-bound Tau remains unrevealed, several scenarios suggested that the negatively charged MT surface tightly binds the positive proline rich region but repels away the negatively charged projection domain. Notably, the affinity of Tau to MT is highly regulated by phosphorylation, particularly at the KXGS motifs. ${ }^{95}$ However, as will be discussed later (Part 1.4.2), Tau hyperphosphorylation compromises this binding ability, triggers Tau aggregation and consequently MT disassembly.

\subsubsection{Tau Pathology in Neurodegenerative Diseases}

Tauopathies are group of neurodegenerative disorders associated to the presence of NFTs in the form of straight or paired helical filaments (PHFs) and are linked to the pathological aggregation of aberrantly phosphorylated Tau protein. ${ }^{96,97}$ The classification is based on (1) the domination of Tau deposition or its coexistence with amyloid plaques, (2) the relative isoform composition of Tau filaments, ${ }^{98}$ (3) cell type and (4) affected regions of the brain (Table 2). ${ }^{52}$ Tau-A $\beta$ concurrence has been detected in Alzheimer's 
disease (AD), Down's Syndrome (DS) and Dementia Pugilistica (DP). On the other hand, the predominance of Tau pathology occurs in other tauopathies that include FrontoTemporal Dementia with Parkinsonism linked to Chromosome 17 (FTDP-17), Progressive Supranuclear Palsy (PSP), Cortico-Basal Degeneration (CBD) and Argyrophilic Grain Disease (AGD). Although the dominating existence of NFTs is intracellular, extracellular tangles (ghost tangles) have been observed in late stages of disease progression.

\section{Table 2: Classification of Tauopathies.}

The classification of Tauopathies is based on cell type, affected regions and the domination of Tau deposition or its coexistence with amyloid plaques. Modified from ${ }^{52,99,100}$.

\begin{tabular}{|c|c|c|c|c|}
\hline Disease & Type & Cognitive Dysfunction & 3R:4R & Protein Involved \\
\hline Alzheimer's Disease & $\begin{array}{l}\text { Hippocampal, medial temporal } \\
\text { atrophy, amlyoid plaques, NFTs }\end{array}$ & Amnestic, cortical & $1: 1$ & \multirow{3}{*}{$\begin{array}{l}\text { Associated with } \\
\text { amyloid deposition }\end{array}$} \\
\hline Down's Syndrome & $\begin{array}{l}\text { Hippocampal, medial temporal } \\
\text { atrophy, amlyoid plaques, NFTs }\end{array}$ & Amnestic, cortical & $1: 1$ & \\
\hline Dementia Pugilistica & $\begin{array}{l}\mathrm{A} \beta \text { and NFT deposition in } \\
\text { cortical and subcortical regions }\end{array}$ & $\begin{array}{l}\text { Amnestic, cortical } \\
\text { following traumatic } \\
\text { brain injury }\end{array}$ & $1: 1$ & \\
\hline Pick's Disease & Frontal atrophy, pick bodies & $\begin{array}{l}\text { Frontal dysexcutive syndrome } \\
\text { Semantic dementia }\end{array}$ & $3: 1$ & \multirow{5}{*}{$\begin{array}{l}\text { Predominantly } \\
\text { Tau pathology }\end{array}$} \\
\hline $\begin{array}{l}\text { Fronto-temporal dementia } \\
\text { with parkinsonism linked } \\
\text { to chromosome } 17\end{array}$ & $\begin{array}{l}\text { Frontal atrophy with tau-positive } \\
\text { neuronal and glial inclusions }\end{array}$ & Frontal behavioral syndrome $\quad 1: 2$, & $1: 1,2: 1$ & \\
\hline $\begin{array}{l}\text { Progressive Supranuclear } \\
\text { Palsy }\end{array}$ & $\begin{array}{l}\text { NFTs in basal ganglia, diencephalon } \\
\text { and brain stem }\end{array}$ & Frontal dysexcutive syndrome & $1: 2-4$ & \\
\hline $\begin{array}{l}\text { Cortico-Basal } \\
\text { Degeneration }\end{array}$ & $\begin{array}{l}\text { Parietofrontal or frontotemporal } \\
\text { atrophy and pallor in substantia nigra, } \\
\text { tau aggregates }\end{array}$ & $\begin{array}{l}\text { Cortical sensory loss } \\
\text { Apraxia }\end{array}$ & $1: 2$ & \\
\hline $\begin{array}{l}\text { Argyrophilic Grain } \\
\text { Disease }\end{array}$ & $\begin{array}{l}\text { Cortical and subcortical granular } \\
\text { changes in neuropil }\end{array}$ & $\begin{array}{l}\text { Memory and personality problems } \\
\text { Limbic dementia }\end{array}$ & $1: 2$ & \\
\hline
\end{tabular}


Of all Tauopathies, AD is the most widespread syndrome, exhibiting protein deposits that consist of extracellular amyloid plaques of A $\beta$ and intracellular neurofibrillary tangles of Tau, ${ }^{101-103}$ both associated to the diagnostic hallmarks of the disorder (Figure 8). The amyloid precursor protein (APP) is an integral membrane protein with unclear functions but its proteolysis produces $\mathrm{A} \beta$. The amyloid hypothesis states that upon mutations in the genes encoding APP and presenilin proteins, the cellular protective role of the enzyme $\alpha$-secretase is replaced by the dominant behavior of $\beta$-secretase. APP is then cleaved and released as SAPP $\beta$. The remaining fragment is processed by $\gamma$-secretase to yield $A \beta$ peptide. Extracellular accumulation of $A \beta_{40}$ or $A \beta_{42}$ ( 40 or 42 residues) then drives hyperphosphorylation and aggregation of Tau. ${ }^{28,104}$ Reports have shown that despite this hypothesis, Tau deposits were detected in the brain before the formation of amyloid plaques. ${ }^{105,106}$ This suggests that the occurrence of Tau inclusions is age-dependent whereas clinical $\mathrm{AD}$ is attributed to the accumulation of $\mathrm{A} \beta .{ }^{107}$ Moreover, the loss in mitochondrial function has been reported in late-onset sporadic AD. ${ }^{108}$ The precise character of these diagnostic hallmark lesions remains an open question, nevertheless, new discoveries continue to identify key structures on their intermediate pathways, kinetics and conceivable intervention. 

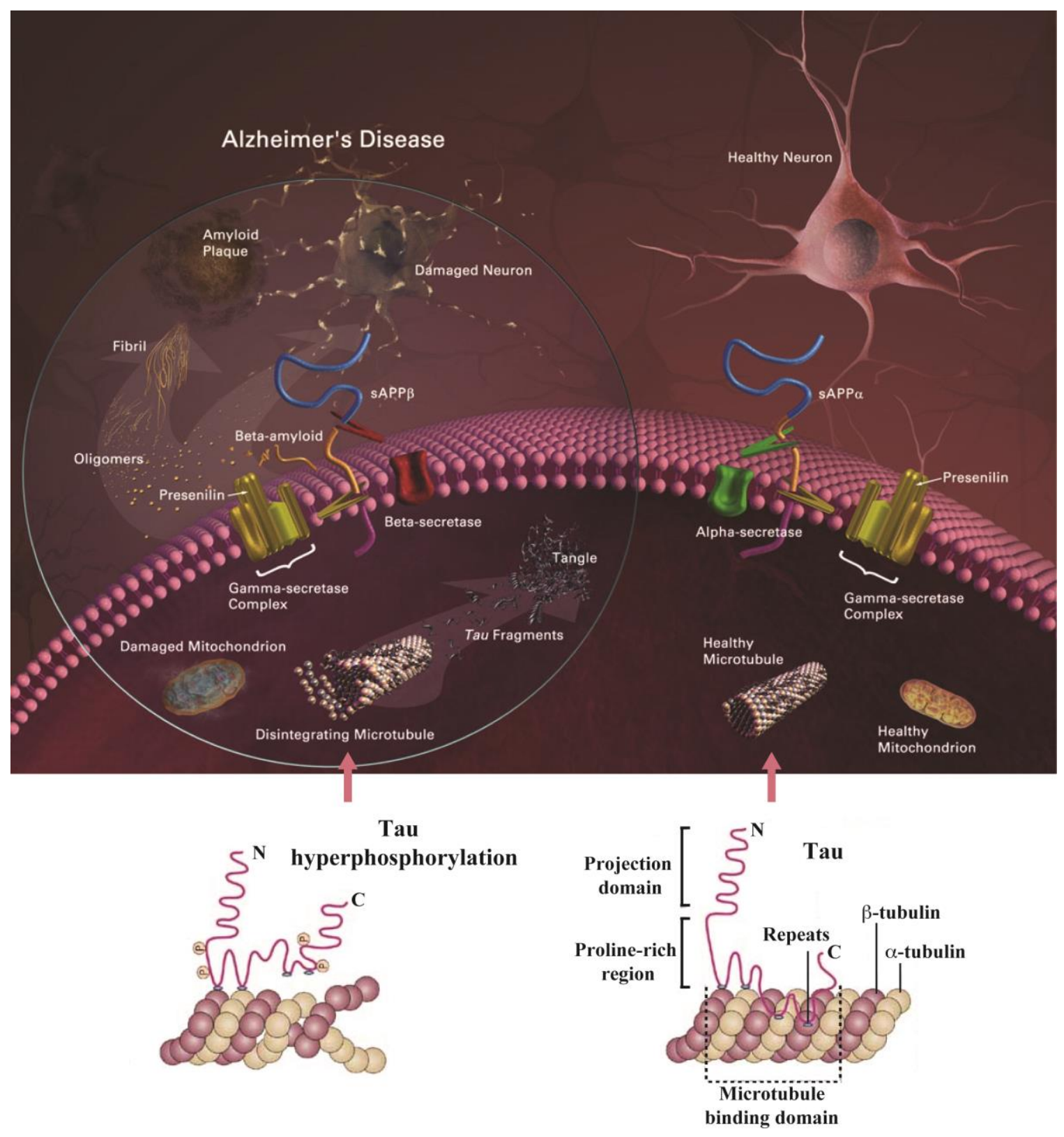

Figure 8: Neuronal Damage during Progression of Alzheimer's Disease.

Mutations in APP and Presenilin initiates the formation of $\beta$-secretase-mediated sAPP $\beta$ and $\gamma$-secretasemediated A $\beta$. This cascade triggers hyperphosphorylation of Tau and consequently its aggregation into NFTs. These events disrupt cellular functions following abnormal cell death. Adapted from National Institutes of Health and ${ }^{109}$. 
Despite the fact that phosphorylation is a major posttranslational modification for regulating MT assembly, hyperphosphorylation of Tau protein is regarded as an early event that precedes filament formation and impairs Tau ability to bind microtubules. ${ }^{110,111}$ Antibodies directed against phosphorylation epitopes have shed light on these consequences. For instance, epitope 12E8 detects the hyperphosphorylation of S262/S356 of pretangled Tau in AD brains and mouse models. ${ }^{112,113}$ A similar study using a triple transgenic mouse model of mutant APP, presenilin and the FTDP-17 mutation P301L suggests that phosphorylation of S214 precedes PHF formation. ${ }^{114}$ More importantly, the serine phosphorylation at the 4 KXGS motifs (S262, S293, S324, and S356) prevents the binding to microtubules ${ }^{115}$ however aggregation into PHFs does not proceed further on. ${ }^{116}$ This suggests that the SP/TP sites of free Tau become more accessible to phosphorylation by the proline-directed kinases rather than promoting aggregation. ${ }^{117}$ As a consequence of destabilizing microtubule assembly, functions and localizations of other subcellular structures such as mitochondria and lysosomes could be altered. ${ }^{118,119}$ Noteworthy, Drosophila flies and mouse models have shown that hyperphosphorylated nonaggregated Tau can be itself toxic. ${ }^{95,120}$

In addition to phosphorylation, other posttranslational modifications regulate the physiological functions of Tau or trigger the pathological pathways. ${ }^{74,121,122}$ This includes:

1. $O$-glycosylation (attachment of oligosaccharide to asparagine, serine or threonine)

2. Glycation (attachment of carbohydrates to lysine)

3. Acetylation (attachment of an acetyl group to lysine)

4. Prolyl-isomerization (conversion between cis and trans conformation)

5. Nitration (attachment of nitrogen oxide to tyrosine)

6. Polyamination (cross-linking between glutamine and lysine) 
7. Deamidation (removal of amide group from asparagine and glutamine)

8. Ubiquitination (binding to ubiquitin)

9. Sumoylation (binding to SUMO1)

10. Oxidation (formation of disulfide bond between two cysteines)

11. Truncation (cleavage at aspartic and glutamic acids)

From the above modifications, truncation of Tau protein has been extensively examined. Truncated species of Tau at three distinctive sites (D13, E391, D421) were detected in the form of PHFs in AD brains. ${ }^{123-125}$ Nevertheless, it is still debatable whether truncation occurs prior aggregation. ${ }^{126}$ Accumulating evidence has demonstrated that hyperphosphorylation precedes Tau truncation, and only after dephosphorylation, aggregation proceeds. ${ }^{127-129}$ On the other hand, Tau oxidation at the two native cysteines (C291, C322) and, consequently, the formation of a disulfide bond has a high impact on aggregation. This will be comprehensively discussed in Part 1.8.3.

To date, no mutations in the Tau gene in chromosome 17 have been correlated to AD. However, gene sequencing revealed several mutation sites linked to FTDP-17. ${ }^{130}$ The identified mutants (K257T, G272V, N279K, $\Delta$ K280, P301L, P301S, S305N, V337M, G389R and R406W) ${ }^{131-135}$ mostly affect the repeat domain and reduce MT binding. ${ }^{97}$ Notably, the point mutant P301L and deletion mutant $\Delta \mathrm{K} 280$ particularly increase the in vitro aggregation into PHFs. ${ }^{134}$ 


\subsubsection{Tau Aggregates}

Noninvasive tools such as positron emission tomography (PET) imaging with plaque-binding and tangle-binding tracers have been developed to diagnose tauopathies. ${ }^{136}$ Electron micrographs of Tau filaments purified from infected human brains or assembled in vitro have implicated three types of morphologies. In AD brains, PHFs constitute more than $90 \%$ of NFTs and the remaining are straight filaments. ${ }^{137}$ Two ribbon-like strands assimilate together to form the helical structure of PHFs with diameters ranging between 8 and $20 \mathrm{~nm}$ and periodicity of $80 \mathrm{~nm} \cdot{ }^{102,138}$ Although most tauopathies share this feature, some contain higher distributions of straight filaments ${ }^{139}$ while others exhibit twisted ribbon-like filaments with irregular periodicity. ${ }^{140}$ During late stages of $\mathrm{AD}$, filaments further adopt average lengths of 300 to $600 \mathrm{~nm} \cdot{ }^{141,142}$ Despite the fact that the sequence of Tau has a relatively low secondary structure content and is disordered, ${ }^{143}$ the filaments contain a highly structured core with repeating cross $\beta$-sheets. ${ }^{144}$ Proteolysis studies have shown that the assembly is mediated by the MT-binding regions but varies among the relative abundance of isoforms. ${ }^{145}$

It is definitely astonishing how a large number of peptides and proteins with no similarities in their primary sequences can self-assemble into amyloid fibrils of common structural features and physico-chemical properties. ${ }^{146,147}$ The conformational switch from natively unfolded to an ordered cross- $\beta$ fibrillar structure is indeed a common pathway recognized in numerous human diseases. ${ }^{26,148,149}$ Extensive studies at atomic-resolution demonstrated the hierarchy and organization of these aggregates. ${ }^{150,151}$ Remarkably, the dominant arrangement of interbackbone hydrogen bonding is the driving force of the structural basis behind $\beta$-sheet self-assembly (Figure 9). In this respect, favored side-chain interactions generate protofilament structures that further re-arrange in filaments. This 
highly structured core of the filaments comprises the repeating cross $\beta$-sheets that are perpendicular to the axis. Following a growth phase, mature fibrils adapt a morphology of defined diameter and periodicity.
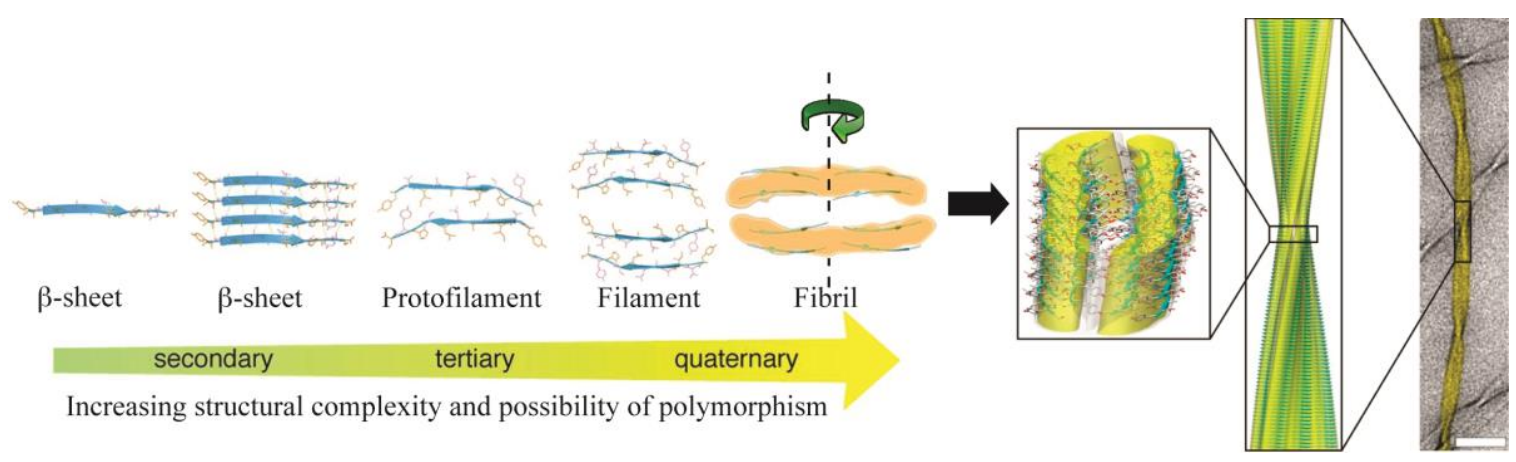

Figure 9: Hierarchical Assembly of Cross- $\beta$ Amyloid Fiber.

The self-assembly of a $\beta$-sheet to an amyloid fibril increases the structural complexity and polymorphism of the transient motifs. The atomic-resolution structure of a triplet fibril is reconstructed from MAS NMR and cryo-EM. Scale bar $50 \mathrm{~nm}$. Adapted from ${ }^{150}$.

In the case of Tau, biochemical and microscopic investigations demonstrated that PHFs isolated from AD brains and assembled in vitro exhibit similar morphologies. ${ }^{152}$ Tau filaments are isolated after incubation of the non-phosphorylated recombinant monomer with polyanions such as RNA, ${ }^{98}$ fatty acids, ${ }^{153}$ glycosaminoglycans, ${ }^{154}$ and heparin..${ }^{155}$ These molecules were proposed to facilitate Tau nucleation either by inducing conformational changes modulated by electrostatic interactions with positively charged residues, ${ }^{154}$ or by exposing charged surfaces. ${ }^{156}$

The course of Tau aggregation from a natively unfolded state to cross- $\beta$ structure ${ }^{157}$ affects a limited region of the repeat domain, ${ }^{158}$ while both amino- and carboxy-terminal halves form the so-called fuzzy coat of the filament. ${ }^{159}$ Protease digestion and solvent accessibility techniques implicated the repeats R2 and R3 in the formation of the PHF 
core. ${ }^{160}$ In particular, the two hexapeptide sequences in the second $\left({ }^{275} \mathrm{VQIINK}{ }^{280}\right)$ and third $\left({ }^{306} \mathrm{VQIVYK}^{311}\right)$ repeats are most deeply buried within the PHF core. ${ }^{90}$ The hydrophobicity and high $\beta$-sheet propensity of these motifs ${ }^{161}$ mediates the intermolecular interactions between the different monomers ${ }^{162}$ and enable self-aggregation. ${ }^{163}$ Moreover, mutagenesis highlighted the essential role of the second hexapeptide ${ }^{306}$ VQIVYK $^{311}$ during filament assembly ${ }^{158}$ with the Y310A and K311D mutations leaving the monomer incompetent to fibrillize. ${ }^{164,165}$

While progress continues to explore the diagnostic hallmark lesions of $\mathrm{AD}$ by deciphering key structures attributed to Tau inclusions, their intermediate pathways and modifications (hyperphosphorylation, proteolytic truncations); the source of toxicity remains an open debate. Recent progress in scientific research has implicated the soluble oligomers to be the most neurotoxic species rather than the insoluble aggregates. ${ }^{166-168}$ These species are extremely transient and labile, imposing a challenge in detecting them. Nevertheless, accumulating efforts have been incorporated to design approaches and develop techniques that can monitor their formation and elucidate their structural properties. During the early stages of oligomeric formation, truncated forms of Tau are indeed composed of monomeric and dimeric subunits that are excessively phosphorylated. ${ }^{169}$ These oligomers exhibit rich $\beta$-sheet conformations and can occur in various $3 \mathrm{R}$ or $4 \mathrm{R}$ isoforms. ${ }^{170}$ Once their size exceeds $20 \mathrm{~nm}$, fibrillar accumulation would follow. ${ }^{171}$ Granular-shaped oligomers isolated from $\mathrm{AD}$ brain tissues and in vitro preparation were found to be assembled of approximately 40 Tau monomeric units in protofibrils and could further build up filaments at effective concentrations. ${ }^{172}$ Oligomers of comparable morphologies were also detected in a Tau P301L transgenic mouse model 
$(J N P L 3)^{173}$ as well as in cellular cultures. ${ }^{174}$ Upon interaction with Tau, chaperones have been reported to decrease the levels of granular oligomers. ${ }^{175}$

With this in mind, the pathology behind Tau aggregation can be outlined through a cascade of intermediates where the monomer switches from a random-coiled to an extended conformation before adopting an ordered $\beta$-sheet structure (Figure 10). ${ }^{74,96,176}$ The events of uncontrolled kinase-mediated phosphorylation, mutations, and abnormal modifications of Tau provoke its dissociation from MT assembly. This loss-of-function induces subsequent impairment of axonal transport, neuronal dysfunction and synaptic degeneration. Under those circumstances, the high levels of unbound Tau in the cytosol then accommodate an aggregation-prone conformation ${ }^{158}$ enriched with $\beta$-sheets. Consequently, Tau dimers assemble through side chain interactions. ${ }^{90}$ Accompanied by a toxic gain-of-function, this nucleation step enables oligomerization to proceed with possible membrane interactions. ${ }^{177}$ The elongation of oligomers to protofilaments is energetically-favored and arranges the intermediates into in-parallel, in register cross $\beta$ sheet structures. Two protofibrils arrange furthermore to form bundles of PHFs that accumulate into NFT deposits. ${ }^{156}$ 


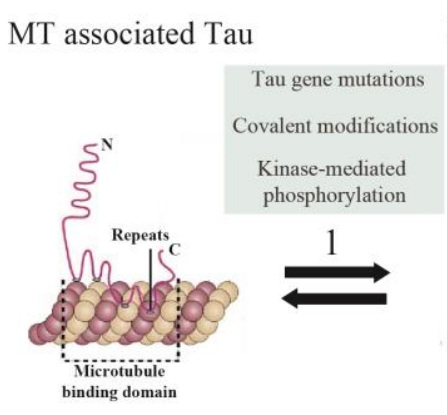

Oligomerization and nucleation

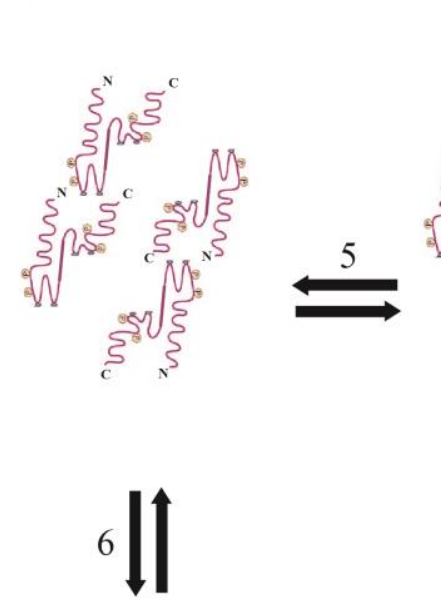

Dimeric formation
Hyperphosphorylated Tau

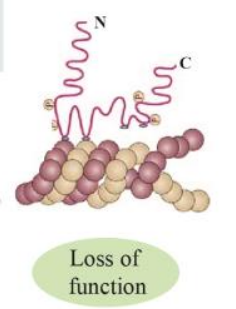

Soluble Tau

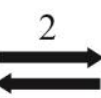

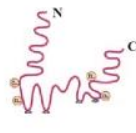

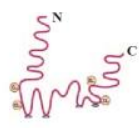

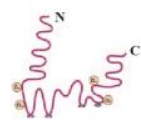

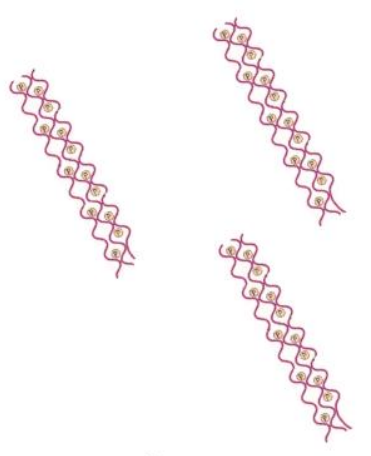

Tau protofilaments
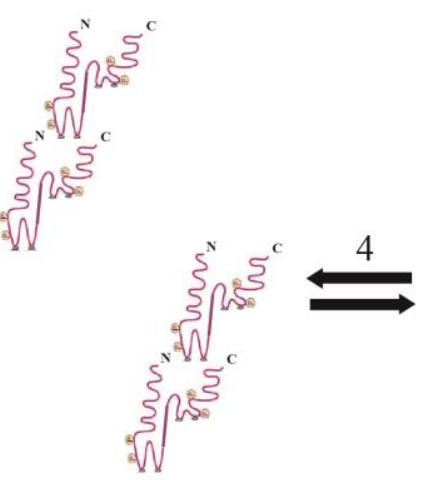

Post-translational modifications

Truncation

Aggregation inducers

Conformational change and $\beta$-sheet enrichment
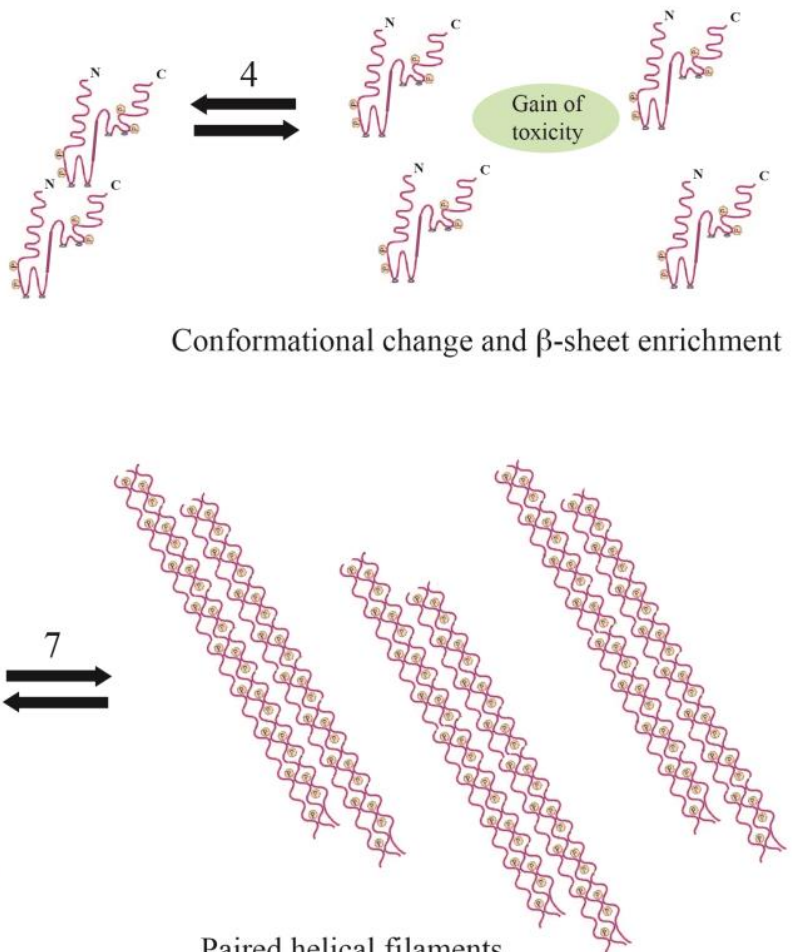

Paired helical filaments

Figure 10: Proposed Cascade for the Pathological Aggregation of Tau.

Uncontrolled phosphorylation, mutations, and modifications dissociate Tau from MT assembly. Unbound Tau then adapts aggregation-prone conformations enriched with $\beta$-sheets and in favor of dimeric formation. This toxic gain-of-function is the basis of a nucleation step through which oligomerization proceed, followed by elongation to protofilaments, and accumulation of PHFs bundles. Modified from ${ }^{74,96,176}$. 


\subsection{Tau-Based Therapeutic Strategies}

Current understanding of the molecular mechanisms and modifications of Taumediated neurotoxicity has established potential targets for the intervention of AD and tauopathies. Tau-based therapeutic approaches aim to regulate phosphorylation, inhibit aggregation, disassemble existing aggregates or divert them into nontoxic forms. Based on their modes of action and disease-modifying potentials, these strategies are classified into six categories that include antiphosphorylation strategies, vaccination, Tau clearance,

isoform control, microtubule stabilizing drugs, and anti-aggregation strategies (Figure 11). ${ }^{117,178,179}$ 


\subsection{Tau-Based Therapeutic Strategies}

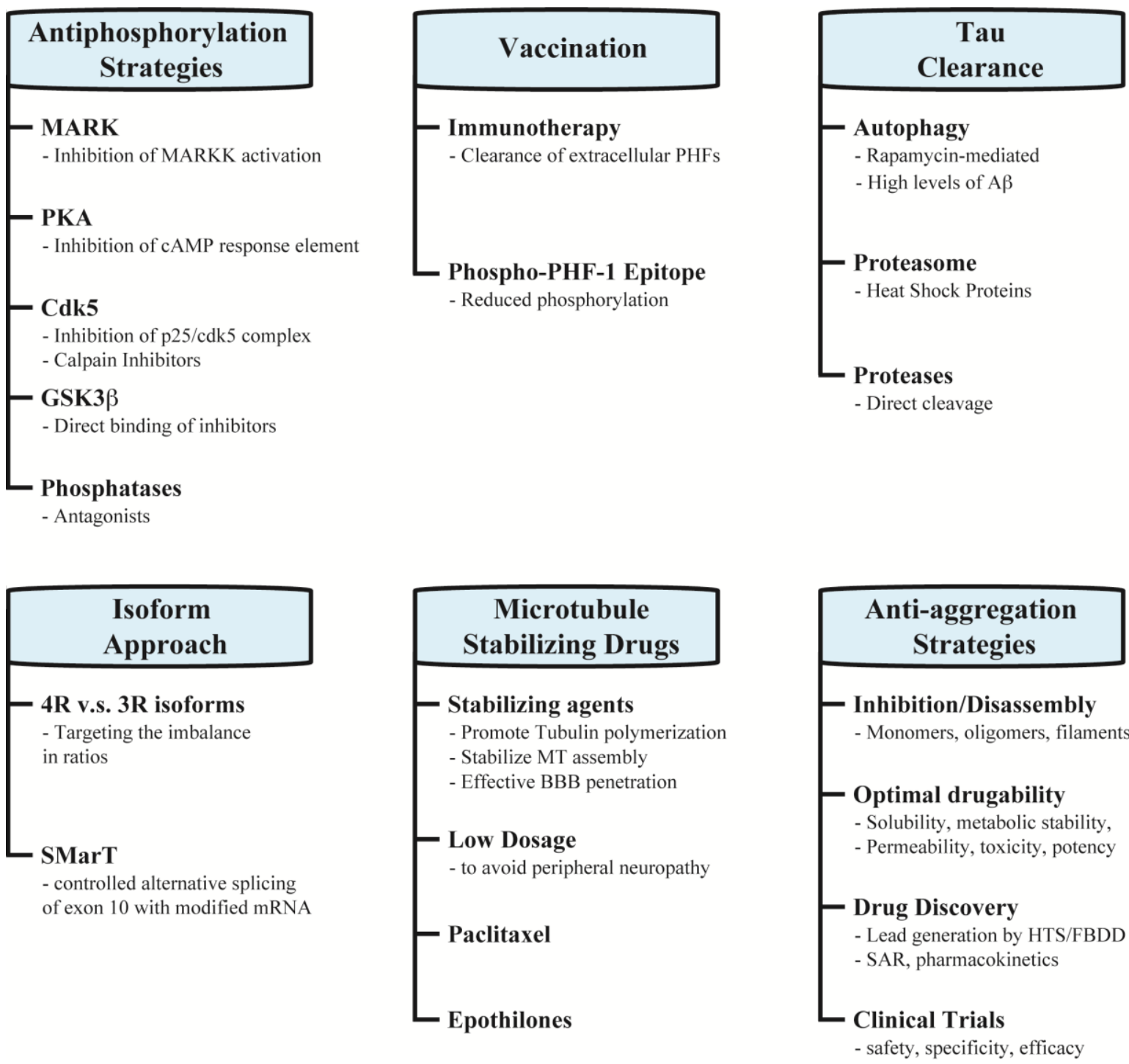

Figure 11: Tau-Based Therapeutic Strategies.

The intervention of $\mathrm{AD}$ and tauopathies aim to regulate phosphorylation, inhibit aggregation, disassemble existing aggregates or divert them into nontoxic forms. These approaches include antiphosphorylation strategies, vaccination, Tau clearance, isoform control, microtubule stabilizing drugs, and anti-aggregation strategies. 


\subsection{Tau-Based Therapeutic Strategies}

\subsubsection{Anti-phosphorylation Approaches}

As already mentioned in $\mathbf{1 . 4 . 2}$, hyperphosphorylation of Tau protein is considered a major posttranslational modification associated to MT disassembly, involved in neurotoxicity and featured in PHF formation. This rises the motivation to interfere with phosphorylation by inhibiting the proline-directed kinases (GSK3 $\beta$, cdk5) and the nonproline directed kinases (PKA, MARK); or alternatively by activating the phosphatases.

MARK is the most abundant calcium/calmodulin-dependent protein kinase associated to KXGS phosphorylation, specifically at S262 and S356. FRET measurements on extracts of AD PHFs confirmed that the active kinase is within close distances to the S262/S356 sites. ${ }^{180}$ MARK-mediated phosphorylation of Tau enhanced the expression of the toxic phenotype in the retina of Drosophila fruit fly but was absent in the S262A/S356A mutants. ${ }^{181}$ MARK becomes functional after the activating kinase MARKK phosphorylates one of its threonines residing in the active site. Since MARK phosphorylation is important during early stages, it is obvious that targeting its activation is of high interest. Indeed, MARK becomes inactive upon MARKK protein-protein interaction with testis-specific protein kinase 1 (TESK1) $)^{182}$ or due to the inhibitory effects of hymenialdisine. ${ }^{183}$

PKA is cAMP dependent kinase that mediates in vitro and in vivo phosphorylation of Tau at S262 and S356 but also extends it to S214. ${ }^{184,185}$ This has an impact on further phosphorylation sites mediated by GSK3 3 . PKI is an inhibitor that has been proposed to displace PKA after binding to its catalytic subunit. ${ }^{186}$ By this mechanism, the gene expression of the cAMP response element is prevented.

P35 is a neuronal-specific activator of cdk5 kinase. Calpain cleaves proteolytically the membrane-bound domain of p35 to generate p25 which itself binds cdk5 and increases the abnormal phosphorylation. ${ }^{187}$ Although targeting cdk5 seems to be a suitable strategy, 
studies on neuronal cell cultures and p35 knock-out mice reported an increased activity of GSK3 $\beta$ upon inhibition of cdk5. ${ }^{188,189}$ Whether preventing p 25 -cdk5 complex association or blocking p 25 generation by targeting calpain, a suitable approach should take into account the deactivation of both kinases. Indeed, the purine compound roscovitine interfered with p25-cdk5 formation and diminished Tau phosphorylation in p25 transgenic mice. ${ }^{187}$ On the other hand, preliminary results of calpain inhibitors screened in AD models were encouraging but with limited penetration to the blood brain barrier (BBB). ${ }^{190}$

GSK3 $\beta$ is one of the two GSK isoforms that are engaged in glucose metabolism, cell proliferation and apoptosis. GSK3 $\beta$ activity is inhibited or triggered by the phosphorylation of S9 and Y216, respectively. ${ }^{191}$ GSK3 $\beta$ phosphorylates Tau in vitro and in vivo at primed epitopes (T231) as well as PHF-1 (S396, S404) and AT8 epitopes (S199, S202, T205). ${ }^{192}$ Overexpression of GSK $3 \beta$ in transgenic mice is directly linked to the high levels of Tau phosphorylation ${ }^{193}$ and enhanced by A.${ }^{194}$ Pyrazolopyrazines, pyrazolopyridines and aminothiazoles decreased Tau aggregation in P301L Tau transgenic mouse model by exhibiting high selectivity for GSK3 $\beta .{ }^{179,195}$ Similar effects have been observed for lithium competing with magnesium GSK3-binding, and with valproate which entered AD clinical trials. ${ }^{196}$

Protein phosphatase-2A (PP-2A) is responsible for the dephosphorylation of Tau protein and the decrease in its activity is directly related to hyperphosphorylation. ${ }^{197}$ Upon inhibition of PP-2A by okadaic acid, levels of hyperphosphorylated Tau increased in cultures isolated from rat brains. ${ }^{198}$ Once hippocampal cultures were treated with memantine, an antagonist for okadaic acid, the main function of PP-2A was recovered. Despite the fact that little is known about PP-2A regulation as a promising therapy, these findings are likely to encourage future investigation. 


\subsection{Tau-Based Therapeutic Strategies}

Since the mentioned kinases and phosphatases are involved in many cellular processes, the identification of inhibitors of high selectivity remains a challenging strategy.

\subsubsection{Tau Vaccination}

Even though the idea of incorporating Tau-directed immunotherapy as a potential approach for the clearance of extracellular PHFs seems convincing, the mechanisms by which Tau-directed antibodies amend the pathology are far from being resolved. ${ }^{199}$ Furthermore, immuno-mediated approach encounters challenges in targeting intracellular tangles as this requires direct uptake of the antibody. Despite the fact that vaccination with full-length Tau triggered encephalitis ${ }^{200}$, active immunization involving the Tau phosphopeptide PHF-1 epitope, on the other hand, reduced Tau phosphorylation and prohibited the pathology in a P301L transgenic mouse model (JNPL3) ${ }^{201}$ However, current vaccinations unveil diverse outcomes with doubts regarding effective penetration of antibodies into the neuronal cytoplasm and glial cells. ${ }^{202}$ Moreover, similar to $A \beta$ immunization neuroinflammation is most likely to occur. ${ }^{203}$ With this in mind, the achievability of Tau vaccination remains unrevealed.

\subsubsection{Tau Clearance}

Yet another approach to prevent the gained neurotoxicity suggests the clearance of Tau aggregates from the cell and possibly its degradation. This would involve two major pathways responsible for protein degradation, autophagosome and proteasome. ${ }^{204}$ This strategy has been reported for oligomers and aggregates of huntingtin, $\alpha \mathrm{S}$, and Tau. ${ }^{205,206}$ The autophagy scheme is simplified as such: the intracellular aggregates are first engulfed 


\subsection{Tau-Based Therapeutic Strategies}

with a double membrane which fuses to the lysosome. The content of the vesicle is then degraded by acidic hydrolases. ${ }^{207}$ Although Rapamycin-induced autophagy was successful in a Drosophila fruit fly model, ${ }^{202}$ however, increased levels of A $\beta$ were observed. ${ }^{208}$

Among the numerous studies on proteasomal degradation of Tau, molecular chaperones were reported to be the most prominent. Specifically, the family of Heat Shock Proteins (Hsp) has occupied considerable attention in protein folding and proteostatis. The inhibition of chaperones Hsp27 an Hsp90 were directly linked to the proteasomal degradation of highly phosphorylated Tau. ${ }^{15,209,210}$ The enhanced behavioral developed in a transgenic mouse model expressing human Tau with one of the FTDP-17 mutations confirmed the role of Hsp90 in the clearance of Tau aggregates. ${ }^{211}$ The interaction of Tau protein and members of the Hsp family will be discussed later in details (Part 1.9). Besides, preliminary studies on Tau degradation implicated the involvement of cytosolic proteases such as Puromycin-sensitive aminopeptidase (PSA), Caspase-3 and Calpain-1. ${ }^{212,213}$

\subsubsection{Isoform Approaches}

4R and 3R isoforms are expressed equally in mature healthy brains but their ratios divert dramatically during Tauopathies. Among the mutations associated to FTDP-17, the dominance of two-fold to six-fold excess of $4 \mathrm{R}$ over $3 \mathrm{R}$ becomes obvious. ${ }^{214}$ Additional diagnoses of brain tissues from PSP, CBD and temporal cortices of AD diseases reported the occurrence of higher ratios of $4 \mathrm{R}$ Tau aggregates. ${ }^{215,216}$ Kinesins and Tau compete for microtubule binding. Bearing in mind the higher affinities of 4R isoforms over 3R towards $\mathrm{MT}$, any isoform imbalance in favor of 4R obstructs the kinesin-regulated cell

trafficking. ${ }^{217}$ Therefore, targeting the imbalance in Tau isoform ratios by controlled 
alternative splicing of exon 10 seems a promising therapeutic approach. Using Tau transfected cells, the modification of mRNA has been successfully demonstrated by a spliceosome-mediated RNA trans-splicing (SMarT) method. ${ }^{218}$ Briefly, the concept is to decrease the ratio of $4 \mathrm{R}$ in favor of $3 \mathrm{R}$ Tau by generating a trans-spliced Tau chimera using a pre-trans-splicing construct that incorporates exon 11, 12 and 13 together with a binding site for intron $9 .{ }^{217}$ However, the poor penetration of RNA into brain cells complicates the adaption of this method to animal models and possible solutions are being investigated. ${ }^{117}$

\subsubsection{Microtubule-Stabilizing Drugs}

The loss-of-function associated to hyperphosphorylated and aggregated Tau is compensated by MT-binding drugs. These molecules are expected to promote tubulin polymerization and stabilize MT assembly. Low doses of these agents are both sufficient and recommended to restore the expected physiological functions and dynamics. MT overstabilization is connected to side effects like peripheral neuropathy. ${ }^{219}$ MT-stabilizing agents exhibited protective responses against Tau-mediated neurotoxicity in cultured neurons. ${ }^{220,221}$ One candidate was found to be Paclitaxel, commercially known as Taxol and a natural compound with established chemotherapeutic properties. When transgenic mice lines with reduced MT density and motor impairments were treated with paclitaxel, the MT density increased and a substantial development in the axonal transport was observed. ${ }^{222}$ But since paclitaxel is not able to cross the $\mathrm{BBB}$, the accompanied improvements were linked to the ligand absorption through the peripheral neuromuscular joints and successively transported to the spinal motor neurons ${ }^{223}$

Another family of compounds is Epothilones. Epothilones A and B are commercial antifungal agents that bind $\beta$-tubulin and promote MT assembly better than paclitaxel as 
was supported by NMR, crystallography and computational investigations. ${ }^{224}$ Further screenings identified Epothilone D, a stabilizing agent with advanced therapeutic properties and enhanced brain penetration abilities. Epitholine D has recently entered a phase $1 \mathrm{~b}$ clinical trial for $\mathrm{AD}$ and related tauopathies. ${ }^{219}$ In addition, numerous natural products or synthetic compounds such as Discodermolide, Dictyostatin, Eleutherobin, and Cyclostreptin have been evaluated for enhanced MT-stabilization with the clear objective of preserving low levels of the drug and effective crossing of the BBB. ${ }^{219}$

\subsubsection{Anti-aggregation Strategies}

Current Tau-based treatment strategies have been directed towards identification of small organic compounds that are able to inhibit or disassemble amyloid aggregates. This approach mainly involves determining molecules that are able to dissociate existing oligomers and fibrils or prevent the formation of their precursors and derivatives. Insights into the mechanisms of action by which these compounds target the protein state (being monomeric, oligomeric or filamentous) reveal the pathological consequences.

The drugability ${ }^{225}$ of a potential inhibitor is evaluated based on:

1. solubility upon oral absorption

2. metabolic stability

3. penetration of the blood brain barrier

4. pharmacokinetic behavior

5. low toxicity

6. suitable half-lives

7. effective excretion 
Design strategies based on fibril -specific fluorescence (Thioflavin S, tryptophan), pelleting and filter assays, cell viability and microscopy (FRET, EM, AFM) have been implemented to identify drug candidates and report their pharmacological properties. For instance, potency defines the drug's evoked response at low concentrations, affinity reflects its binding ability and efficacy relates the receptor occupancy to the drug's response. Hence, the three terminologies are interconnected and are evaluated by the term half maximal effective concentration $\left(\mathrm{EC}_{50}\right)$. Similarly, the half maximal inhibitory concentration $\left(\mathrm{IC}_{50}\right)$ and disassembly concentration $\left(\mathrm{DC}_{50}\right)$ respectively report the effective concentrations required to inhibit the assembly into aggregates or disassembly of preexisting aggregates. The equilibrium dissociation constant $\left(\mathrm{K}_{\mathrm{d}}\right)$ of a compound is equally important and relates the concentration needed to reach half maximal saturation of the binding sites. Cytotoxicity is commonly monitored by lactate dehydrogenase (LDH) assay that reports on the leakiness of membranes in degenerating cells.

The process of drug discovery consists of stages for identifying candidates that further undergo structural and functional optimization to improve their biological functions. Once the target system or protein is defined, selected chemical compounds and fragments are screened using primary assays. This lead generation step can adapt two approaches: high throughput screening (HTS ${ }^{226}$ or fragment-based drug design (FBDD). ${ }^{227}$ While HTS screens a large library of compounds that bind their targets with medium affinity $\left(\mathrm{K}_{\mathrm{d}}<1 \mu \mathrm{M}\right)$ but low ligand efficiency, FBDD targets a smaller number of molecules and fragments of higher ligand efficiency but weak affinity $(\mu \mathrm{M}-\mathrm{mM})$. Recently, cell-based high content screens (HCS) have been developed to assay the biological events in the presence of the candidates. ${ }^{228}$ 


\subsection{Tau-Based Therapeutic Strategies}

The identified hits undergo further optimization to enhance their structure-activity relationships (SAR) and improve their selectivity, potency and pharmacokinetics. NMR is one of the most suited methods that provides structural information for rational lead design. Ligand-mediated screenings based on SAR by NMR have indeed augmented considerably the number of screened libraries and expanded the confidence in FBDD. ${ }^{229}$

The final time-demanding and expensive stage is clinical trial. This covers the broader scope of drugability, patient's safety and drug's efficacy to the target. Drug discovery remains a challenging research field with more than $90 \%$ of the candidates entering clinical trials are bound to failure and very few drugs successfully accomplish the long journey from lab bench to bedside. Section $\mathbf{1 . 6}$ will present in more details the up-todate cutting edge research of aggregation inhibitors aimed at treatment of Tau aggregates.

\subsection{Aggregation Inhibitors}

Conceptually, the inhibition of Tau assembly is certainly an appealing approach for treatment of tauopathies where engineered compounds would selectively target Tau assemblies. However, this methodology encounters abundant practical challenges and is bound to high percentages of failure such as low penetration of the BBB, provoked toxicity, and overestimated selectivity. Nevertheless, the development of effective protocols to identify inhibitors through HTS is established by incorporating several biophysical techniques together with cell and animal models.

Fibrillar Tau deposits are stained by dyes such as Thioflavin T and S (ThT, ThS) that exhibit enhanced fluorescence upon binding to cross $\beta$ structures. ${ }^{230}$ This assay was combined with pelleting and filter experiments, cell viability and microscopy to identify 
chemical compounds in primary screenings. Using ThS fluorescence assay, Sarkosyl insolubility and EM, 42 compounds from 9 chemical classes were screened for their plausible inhibition of in vitro heparin-induced full length Tau assembly. Phenothiazines, polyphenols and porphyrins were among the classes as potential inhibitors. ${ }^{231}$ In a largerscale screening of 200,000 compounds, similar assays were conducted with 3R and 4R Tau fragments (K19 and K18) to evaluate their inhibition for aggregation and their induced disassembly. ${ }^{232}$ From the primary screenings, 77 compounds of the identified hits were distributed among the organic families $N$-phenylamines, anthraquinones, phenylthiazolylhydrazides, and rhodanines. These classes were further analyzed by a handful of secondary assays including toxicity and activity in cells. Noteworthy, quinoxalines and pyrimidotriazines were among the newly identified classes in an HTS library of 51,000 compounds screened for inhibition of heparin-induced fibrils. ${ }^{233}$

Numerous screenings of small organic molecules revealed common features of potential aggregation inhibitors. Most importantly was the action of inhibitors to redirect the amyloid fibril formation to off pathway nontoxic oligomers. ${ }^{234}$ On the other hand, the role of aromatic interaction was found to be equally important in driving amyloid assembly as well as a recognised inhibition mechanism. ${ }^{235}$ Self-aggregation is a common feature in many organic classes, which is influenced by concentration, $\mathrm{pH}$, ionic strength, temperature, solvent polarity, and structure. ${ }^{236}$ For instance, the high concentration of a compound candidate and consequently its aggregates enhanced Tau fibrillization in one case $^{156}$ but in another case, it inhibited amyloid fibril formation. ${ }^{237}$ The structural, physical and chemical properties of the most promising classes of organic compounds together with their biological applications are discussed in more details below (Table 3 and part 1.6.11.6.10). 


\subsection{Aggregation Inhibitors}

Table 3: Chemical Structures of Screened Classes.

$\mathrm{IC}_{50}$ values obtained from ${ }^{60,231,233,238-240}$.

Chemical Class General Structure

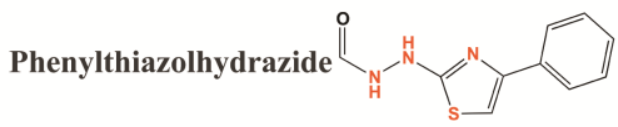

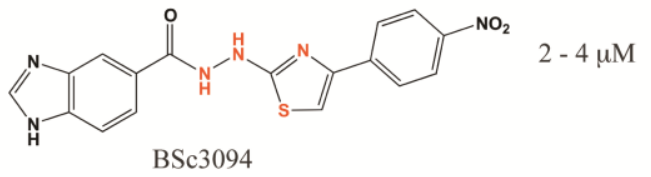

N-Phenylamine<smiles>c1ccc(Nc2ccncn2)cc1</smiles>

Anthraquinone<smiles>O=C1c2ccccc2C(=O)c2ccccc21</smiles>

Polyphenol catechin<smiles>O[C@H]1Cc2ccccc2O[C@H]1c1ccccc1</smiles><smiles>Cc1nc(Nc2ccccc2C(=O)O)nc(Nc2ccccc2C(=O)O)c1[N+](=O)[O-]</smiles>

$8-10 \mu \mathrm{M}$<smiles>Cc1cc(O)c2c(c1)C(=O)c1cc(O)cc(O)c1C2=O</smiles>

$2-4 \mu \mathrm{M}$<smiles>CC(=O)C(=O)Cl</smiles>

$1.8 \mu \mathrm{M}$ 


\subsection{Aggregation Inhibitors}

Table 3: Chemical Structures of Screened Classes. (Continue)

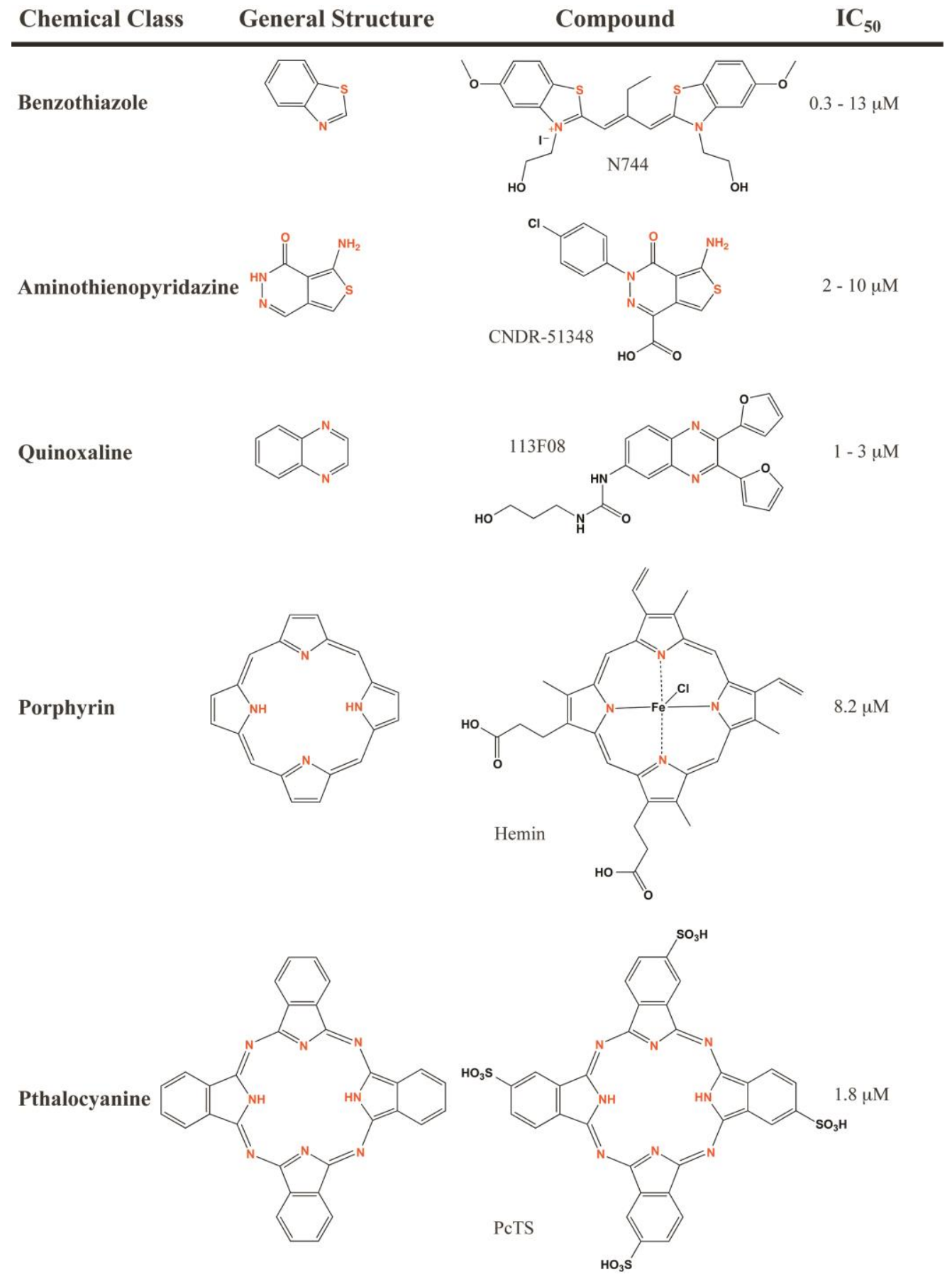




\subsubsection{Phenothiazines}

Phenothiazine is a hetero tricyclic molecule with a central thiazine ring and two outer benzene rings (Table 3). Derivatives of the parent compound are pharmaceutically significant and are characterized by the type of substituents attached at the peripheral rings. Many phenothiazines are commercial drugs with antipsychotic (Chlorpromazine), antihistaminic (Promethazine), antimalarial (Chloroquine), and cancer chemopreventive effects $^{241-243}$ targeting dopaminergic and neuronal receptors of the central nervous system $\left(\alpha\right.$-adrenergic, serotonin, histamine). ${ }^{244-246}$ The amphiphilic character of phenothiazines and high degree of ring lipophilicity enable them to cross the BBB. ${ }^{247}$ Phenothiazines have been also reported for their inhibitory and anti-proliferative effects of calmodulin (CaM) and PKC activities. ${ }^{248}$

The widespread use of a remarkable phenothiazinium salt in diverse medical applications has proved the clinical efficacy of this class in drug research. This compound is [3,7-bis(dimethylamino)phenothiazinium chloride],better known as Methylthionine hydrochloride or Methylene Blue (MB). MB was first synthesized in 1876 by the German chemist Heinrich Caro at BASF. Soon after, dye derivatives were applied in microscopy for staining biological tissues, with distinct chemical classes exhibiting different selectivity to cell types. In 1891, the German biologists Paul Guttmann and Paul Ehrlich suggested the physiological and clinical relevance of histological stains and discovered the treatment of Malaria with MB. ${ }^{249}$ Nowadays, MB is regarded as the therapeutic agent from which the chemical development of antimalarial drugs initiated. Part 1.8 is dedicated to the physical and chemical properties of MB along with its biological applications. 


\subsubsection{Rhodanines}

Rhodanines are derivatives of thiazolidine with a central core regarded as a template for engineering biologically active molecules (Table 3). For instance, Epalrestat, is a well-known inhibitor of aldose reductase that delays the progression of diabetic neuropathy. ${ }^{250}$ The rhodanine-based derivative 2-thioxothiazolidin-4-one was identified during a high-throughput screen $^{232}$ and secondary assays were then performed to characterize the assembly and disassembly of Tau aggregates based on $\mathrm{IC}_{50}$ and $\mathrm{DC}_{50}$ values. ${ }^{238}$ Insights into the SAR was obtained by systematically replacing the rhodanine core and the branched substituents with different functional groups. The thioxo group of the rhodanine core maintained the highest potency due to its low electronegativity and high potential for hydrogen bonding. Moreover, esterification of the branched carboxylic acid reduced the disassembly, while increasing the linker with the core up to two carbons showed higher inhibitory effects. On the other hand, variations of the side chain on the furan heterocycle reduced the potency. Therefore, the relevance of the thioxo group of the core, the optimal chain length connecting the carboxylic acid and the role of the electronrich furan altogether establish the observed pharmacological properties. The screened compounds were further investigated in murine neuroblastoma N2a cells to characterize inhibition and cytotoxicity with ThS fluorescence and LDH assays. Rhodanines at subconcentration levels $(100-600 \mathrm{nM})$ were found to inhibit Tau aggregation and PHF disassembly without exhibiting cytotoxicity or interference with the stability of MT. ${ }^{238}$ However, rhodanines should be further investigated for their ability to cross the BBB. 


\subsubsection{Phenylthiazolhydrazides}

Phenylthiazolhydrazides (PTH) were predicted by in silico screenings ${ }^{251}$ and 49 compounds were selected to verify the lead structure. The most active functional groups were optimized for SAR and the overall biological activity upon incubation with 4R K18 fragment. BSc3094 was reported to be the most potent PTH as depicted by a generated pharmacophore model ${ }^{252}$ and saturation transfer difference (STD) NMR experiments. ${ }^{251}$ The compound contains a hydrophobic region within the thiazole ring (Table 3), the carboxyl amide is a hydrogen-bond acceptor and of the two aromatic rings, the benzimidazole ring is more hydrophobic, favors $\pi$-stacking interactions and represents the binding epitope as accessed by STD NMR. Although the cell activities of rhodanines and PTH were comparable, The latter showed lower in vitro potency and higher cytotoxicity, suggesting a higher cell permeability. ${ }^{239}$

\subsection{4 $\mathrm{N}$-Phenylamines}

$N$-phenylamines were also identified within the HTS approach ${ }^{232}$ and showed similar activities as rhodanines, but with lower in vitro pharmacological potency. ${ }^{253} \mathrm{EM}$ micrographs of the treated K19 filaments revealed shortening and breaking morphologies in a dose-dependent fashion. The identified B4A1 and B4D3 (Table 3) were further tested for cell viability and toxicity using neuronal cell lines N2a and expressing four repeat domains of Tau (K18) in wild type and the pro-aggregation mutant K18 $\Delta 280$. The selected compounds inhibited and disassembled Tau aggregates in cells and reduced the LDH release. The drugs efficiencies for inhibition and disassembly were reported to be 60 to $70 \%$ and 40 to $45 \%$, respectively. Toxicity was relatively low at the used concentrations, 
however, the determination of the effective cellular concentration was uncertain. The SAR of this class remains unclear and requires further investigation.

\subsubsection{Anthraquinones}

Anthraquinones incorporate a tricyclic aromatic ring system of an anthracene with two keto groups located on the central ring (Table 3). Hydrogen bonding is enhanced in the presence of one or more aryl hydroxyl groups. Several hydroxyanthraquinone derivatives, known as anthracenediones, are well-established medicines that include laxatives (emodin), antimalarial (rufigallol) and chemotherapeutic (mitoxantrone, daunorubicin and adriamycin) drugs. HTS for K19 PHF inhibition reported the hydroxyanthraquinones derivatives emodin, daunorubicin and adriamycin as potential candidates. ${ }^{232}$ The study also included two compounds identified as hydroxyanthraquinone PHF016, containing 4 hydroxyl groups, and the benzophenone derivative PHF005. The first three compounds showed dose-dependent inhibitory effects toward hTau23 and hTau24 PHF formation (50\% at $0.1 \mu \mathrm{M}$ and $90 \%$ at $60 \mu \mathrm{M})$ whereas PHF016 and PHF005 had low significance. When incubated with 3R hTau23 PHFs, a similar trend was observed: emodin, daunorubicin and adriamycin were most potent with $\mathrm{DC}_{50}$ values of 7 to $13 \mu \mathrm{M}$. By contrast, PHF016 and PHF005 achieved better results than the others with $\mathrm{DC}_{50}$ values between 10 and $40 \mu \mathrm{M}$. The results of subsequent assays (ThS fluorescence, tryptophan fluorescence shift, EM and filter trap) have all emerged into one conclusion: the compounds are more efficient in inhibiting de novo formation of filaments rather than in depolymerizing them. ${ }^{232}$ However, extending incubation times up to 28 days revealed that the disassembly is time-dependent. Interestingly, these compounds do not interact with the 
assembly of microtubules and are specific to the pathological aggregation. Of the 5 compounds tested in N2a cell model expressing K18 $\Delta$ K280, emodin was most effective by suppressing the aggregates in the treated cells.

\subsubsection{Polyphenols}

Polyphenols are natural and synthetic organic compounds that exhibit multiple phenol structural units. Polyphenols occur in plants and dietary supplements (red wine, green tea, olive oil) with established antibiotic and neuroprotective properties. The polyphenolic compound extracted from green tea, (-)epigallocatechin-3-gallate (EGCG), exhibits potent antioxidant, therapeutic and anti-carcinogenic effects. The chemical structure of EGCG incorporates pyrogallol (benzene-1,2,3-triol) and gallic acid (3,4,5trihydroxybenzoic acid) that are connected to a catechin-like subunit (Table 3). EGCG provokes essential bioactivities in cellular processes such as MAPK, Wnt signaling, and proteasome inhibition. ${ }^{254-256}$ More importantly, EGCG has been intensely investigated for its neuroprotective actions in neurodegenerative pathologies, specifically as a potent inhibitor for $\alpha \mathrm{S}, \mathrm{A} \beta$, polyglutamine and Tau fibrillization pathways. EGCG redirects $\alpha \mathrm{S}$ amyloid formation to off pathway non-toxic stable oligomers ${ }^{254}$ that are impaired in the pore formation of planar lipid bilayers. ${ }^{257,258}$ It binds directly to monomeric $\alpha \mathrm{S}$ and stimulates the assembly of spherical oligomers with average diameters of $20 \mathrm{~nm} .{ }^{259}$ These off-pathway oligomers are incompetent of forming amyloidogenic $\beta$-sheet structures and are nontoxic to mammalian cells. Additionally, immunohistochemical analysis of coronal sections from transgenic mice has revealed that EGCG reduces $A \beta_{40}$ and $A \beta_{42}$ deposition ${ }^{260}$ and suppresses sarkosyl-soluble phosphorylated Tau isoforms. ${ }^{261}$ EGCG was also 
investigated in Huntington's disease whereupon its oral administration in drosophila flies, it acts as an antagonist for the polyglutamine-based huntingtin protein and interferes with toxic aggregation. ${ }^{262}$ SAR-based analyses have investigated the impact of EGCG derivatives. Both hydroxyl-containing moieties, pyrogallol and gallate, are indeed essential for inhibition since a gallate-free catechin proved to be inactive.

Despite the fact that EGCG penetrates the BBB in mammals with no adverse effects, ${ }^{263}$ several contradicting results have questioned its candidacy for clinical trials. These studies have shown that EGCG exhibit a relatively poor bioavailability and is modified through biotransformation reactions such as methylation or glucoronidation. ${ }^{264}$ The binding of EGCG to unfolded regions is nonspecific with consequences of interfering with any of the numerous signaling proteins that possess such regions. ${ }^{265}$

\subsubsection{Benzothiazoles}

Benzothiazoles are compounds with a stable electron-withdrawing aromatic system that provides hydrophobic interactions. ThT and ThS are examples of benzothiazole derivatives. Thiacarbocyanine is a class of compounds with two benzothiazole moieties, better known as cyanine dyes (Table 3). A library of screened classes of inhibitors has suggested compound 3,3'-bis( $\beta$-hydroxyethyl)-9-ethyl-5,5'-dimethoxythiacarbocyanine iodide, better known as N744, as a candidate that inhibits arachidonic acid-induced fibrillization of full-length Tau and causes disaggregation of pre-formed Tau filaments. ${ }^{266}$ Despite the incubation of N744 with $\mathrm{A} \beta$ and $\alpha \mathrm{S}$ at similar sub-stoichiometric concentrations, aggregation proceeded independently thus confirming the high selectivity to Tau. However, N744 was determined to have a biphasic activity. At low concentrations, the domination of monomeric and dimeric forms of N744 inhibit Tau aggregation with low 
$\mathrm{IC}_{50}$ values $(0.3-13 \mu \mathrm{M})$. At higher concentrations, N744 self-assembles in H-aggregates of columnar stacks and enhances Tau fibrillization. ${ }^{267}$ This suggested that once the concentration of the binding target is low, dimer formation of N744 is stabilized and seems to be the responsible species behind the inhibitory effect. On the other hand, the ligand's higher order aggregates fail to inhibit Tau fibrillar formation or disaggregation. Thus, confirming that these concentrated aggregates are the driving force behind the protein fibrillization and is in line with the fact that proteins with extended $\beta$-sheets influence cyanine aggregation. ${ }^{268}$ Surprisingly, in other cases, it was the formation of compound aggregates that had the inhibitory role on amyloid fibril formation. ${ }^{237}$ In a complementary study, characterization of a cyclic bis-thiacarbocyanine containing two thiacarbocyanine moieties showed higher potency than thiacarbocyanine N744 suggesting that the mode of inhibition relies on the molecule's multivalency rather than self-stacking. ${ }^{269}$ The identification of these inhibitors set the basic template for designing multivalent molecules. The necessity of prolonged aromatic systems and enhanced solubility while optimizing length, flexibility and hydrophobicity of the linkers all together provide higher potency.

Recently, a study reported the synthesis of $N, N^{\prime}$-alkylene bis-thiacarbocyanines with different chain lengths to examine their impact on potency and extension of the structure-activity relationship. ${ }^{270}$ The linker length of four methylene units preserved the open monomer conformation and resulted in the highest efficiency. Despite the progressive improvement in the synthesis and effects of these molecules, this class of inhibitors has not yet been tested in vivo or in animal models. 


\subsubsection{Aminothienopyridazines}

Aminothienopyridazines (ATPZ) are heterocyclic compounds containing a pyridazine-derived ring fused to a thiophene moiety (Table 3). This class of compounds was first identified in a quantitative HTS of 292,000 compounds for possible inhibition of K18 P301L aggregates. ${ }^{271}$ Secondary screenings of selected ATPZs derivatives showed that these compounds do not interfere with MT assembly and are selective for Tau but less effective towards $A \beta_{42}$. In an extended study, ATPZs were evaluated for their in vitro and in vivo efficacy as well as SAR by designing derivatives that took into account plausible BBB permeation. ${ }^{272}$ Notably, the amino group on the thiophene ring is important for biological function, whereas alkylation were unfavorable on both rings. Recently, ATPZs were described as agents that facilitates oxidation of cysteines in Tau. ${ }^{273}$ This identified the mode of action by which ATPZ promote intermolecular and intramolecular disulfide bond formation in $3 \mathrm{R}$ and $4 \mathrm{R}$ isoforms, respectively. Although this retains $4 \mathrm{R}$ Tau in its monomeric form, however ATPZ enhances the dimeric assembly in 3R Tau which act as aggregation seeds. Since, these dimers are still capable of fibrillization, the application of ATPZ in treating AD does not seem appropriate.

\subsubsection{Quinoxalines}

Quinoxalines are heterocyclic compounds that comprise a benzene ring and a pyrazine ring in their core structures (Table 3). Quinoxalines such as Actinoleutin and Echinomycin are known for their pharmaceutical and antitumoral properties. In a largescale HTS library of 51,000 compounds, primary assays were conducted to evaluate the inhibition of heparin-induced Tau fibrils. ${ }^{233}$ The study reported several classes that were already nominated as potential inhibitors (phenothiazines, porphyrins, anthraquinones) in 
addition to newly identified classes (quinoxalines and pyrimidotriazines). Of all remaining candidates, 2,3-di(furan-2-yl)-quinoxaline (113F08) exhibited $\mathrm{IC}_{50}$ values in the range of 1 to $3 \mu \mathrm{M}$. Upon inspection of different quinoxaline derivatives for improved SAR, the potency of the candidate $113 \mathrm{~F} 08$ was found to be highly dependent on the presence of the two furanyl moieties. To date, there has been no further in vitro or in vivo studies on this particular class.

\subsubsection{Porphyrins}

Porphyrins are aromatic heterocyclic macrocycles that belong to the cyclic tetrapyrrole family. The structural basis of a porphyrin incorporates four pyrrole subunits interconnected at their alpha carbon atoms via a methine bridge to include a total of $22 \pi$ electrons (Table 3). The four central nitrogen atoms provide the chromophore for a multitude of metal complexes including iron, magnesium, cobalt, nickel, copper and aluminum. The metal ion of these metalloporphyrins usually has a charge of $2+$ or $3+$. The iron-containing porphyrin Heme is a cofactor of the protein hemoglobin. Porphyrins have established applications that include dye industry, catalysts, sensitized solar cells, and photodynamic therapy. The metalloporphyrins Hemin and Hematin, structurally related to heme, have been reported to interfere with $A \beta_{42}$ oligomerization with the most likely forming iron-histidines complexes via the $\pi$-electrons of the imidazole ring.

Phthalocyanine (Pc) belongs to the family of cyclic tetrapyrroles where the hydrophobic central structure accommodates a porphyrin moiety except the imines replace the methines (Table 3). The resulting central 16-membered ring is hydrophobic, highly conjugated and contains a total of 8 nitrogen atoms, 4 of which can coordinate with metal 
ions. In contrast to the unsubstituted pyrroles in porphyrins, Pc contains four isoindoles (benzo-fused pyrroles) that can carry various functional groups at the peripheral outer benzo substitution positions. In addition to the substitution of hydrogen atoms by halogens, hydroxyl, amino, alkyl, aryl, thiol and nitro functional groups, Pc exhibits a sulfonation degree from 0 up to 4, with Phthalocyanine tetrasulfonate (PcTS) accommodating four negatively charged sulfonate groups (Table 3). In addition to their wide employment in textile dyeing and organic pigments, Phthalocyanines have gained industrial applications as photoconducting agents in photocopying devices, chemical sensors, optical storage devices, and catalysts for numerous chemical reactions. Of most importance is their role in photodynamic therapy. ${ }^{274,275}$ This class of cyclic tetrapyrroles has stronger absorbance at longer wavelengths than porphyrins. The photodynamic properties of PcTS derivatives are currently under investigation for possible cancer treatment. Their preferential uptake in cancerous cells and their high fluorescence yields both display advantages for tumor detection using time-resolved imaging techniques. Of all PcTS-metal complexes, chloroaluminum Phthalocyanine Tetrasulfonate (AlPcTS) is most hydrophilic and possesses significant photodynamic effects. The administration of phthalocyanines to animals results in their localization in the plasma membranes and subcellular organelle membranes such as nuclei, mitochondria, lysosomes, and microsomes. It is therefore accepted that membranes are principal targets of phototoxic effects associated with Pcmediated photodynamic processes. 


\subsection{Phthalocyanine Tetrasulfonate}

\subsection{Phthalocyanine Tetrasulfonate}

The term Phthalocyanine originates from Greek (naptha means rock oil and cyanine means dark blue) and was first used in 1933 by Sir Reginald Linstead to describe this class of macrocyclic compounds. ${ }^{276}$ Scottish Dyes Ltd. pioneered the application of this family and was granted the first patent of phthalocyanines. Years later, X-ray crystallography confirmed the structure of these compounds.

\subsubsection{Physical and Chemical Properties}

Phthalocyanines exhibit various photophysical and chemical properties based on the nature of peripheral substituents or axial ligands to the chelated central metal ion. During PcTS synthesis, sulfonation may occur on adjacent or opposite rings and hence preparations usually contain a mixture of regioisomers in undefined relative amounts between mono and tetrasulfonate. ${ }^{277}$ This possible ambiguity is overcome by use of the same preparation during spectral studies and cell cultures or by further batch purifications.

Following the displacement of two hydrogen atoms from the central nitrogens in PcTS, the metal ion occupancy neutralizes one $\left(\mathrm{Fe}^{3+}, \mathrm{Mn}^{3+}, \mathrm{Al}^{3+}\right)$ or both $\left(\mathrm{Ni}^{2+}, \mathrm{Cu}^{2+}, \mathrm{Zn}^{2+}\right.$, $\mathrm{V}^{4+} \mathrm{O}$ ) of the resulting negative charges. The metal type favors a preferred coordination stereochemistry. For instance, $\mathrm{Ni}^{2+}$ and $\mathrm{Cu}^{2+}$ form square planar complexes, with the metal atom being coplanar to the four nitrogens whereas $\mathrm{Fe}^{3+}, \mathrm{Al}^{3+}$, and $\mathrm{V}^{4+}$ establish square pyramidal structures, wherein the metal lies outside the plane of the nitrogens. $\mathrm{Fe}^{3+}$ can additionally bind two axial ligands, one on each side of the macrocycle and retain the coplanarity with the nitrogens. Although the nature of the metal does not seem to be a 


\subsection{Phthalocyanine Tetrasulfonate}

critical determinant of the inhibitory activity, however, it influences the photophysical properties in terms of triplet quantum yield and lifetime. ${ }^{278}$

Like several organic dyes, PcTS aggregates in solution due to the non-covalent attractive interactions between the aromatic rings that drive the coplanar association of PcTS monomer to dimers and further to higher order complexes. Physical effects such as metal ion occupancy, ionic strength, temperature, and concentration dependency may alter this molecular self-assembly. The peripheral hydrophilic sulfonate groups promote the solubility of the whole system in aqueous solutions, but also trigger coulomb repulsive forces due to the negative charges. The non-covalent $\pi-\pi$ interaction is caused by intermolecular overlapping of p-orbitals in $\pi$ conjugated systems, with large orbital dependent contribution to the dispersion component of Van der Waals forces. These abundant delocalized $\pi$ electrons favor flat polycyclic aromatic systems to adopt stacked arrangements. Other stacking forces include hydrogen bonds, charge transfers and dipoledipole interactions. On the other hand, electrostatic forces might considerably weaken this effect in aromatics but does not entirely cancel it. This oligomerization phenomenon arises from the planarity of the system with $\pi-\pi$ stacking associating the aggregates perpendicular to the axis of the plane. Depending on the geometry of the stacked arrangement and relative orientation, the resulting structures can adapt two assemblies, $H$ - (hypsochromic) or $J$ (Jelley) aggregates. ${ }^{278}$ Although both forms define the linear stacking of coplanar molecular units with a distance of $3.3 \AA$, the angle between the long molecular axis and the stacking axis, known as the tilt angle $\alpha$, distinguishes $H$-oligomeric $\left(\alpha=90^{\circ}\right)$ from $J$ polymeric $\left(\alpha<10^{0}\right)$ aggregates.

Monomeric Pcs have characteristic absorption spectra with two absorption peaks, one appearing at approximately $350 \mathrm{~nm}$ (known as Soret peak) and another around 600 


\subsection{Phthalocyanine Tetrasulfonate}

$\mathrm{nm}$. Aggregation is spectroscopically detected and depending on the direction of the relative orientation, the absorption of the Soret region occurs at shorter ( $H$ - aggregates) or longer wavelengths ( $J$-aggregates). Aggregation is also recognized by NMR spectroscopy. The concentration-dependent association of molecules in solution perturbates the diamagnetic shielding of their constituent nuclei. As a result of the coplanar stacking, ring currents reinforce the magnitude of the diamagnetic shielding and trigger upfield chemical shifts in resonances of nuclei located near the $\pi$ electron macroring, mostly the peripheral aromatic protons (Figure 12). Aqueous media tend to promote PcTS self-stacking, whereas detergents, plasma proteins and organic solvents such as DMSO stabilize the monomeric species. $^{279}$

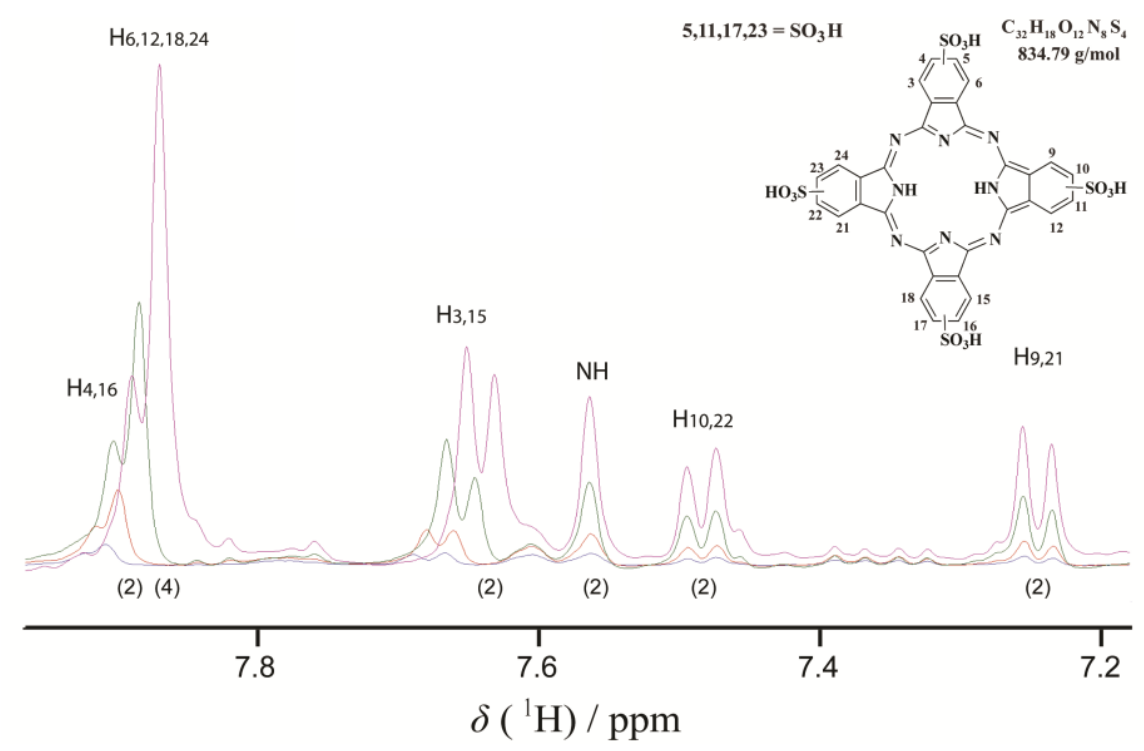

Figure 12: The Self-stacking of PcTS as Detected by NMR Spectroscopy.

Structural elucidation of PcTS and evaluation of its concentration-dependent self-aggregation from ${ }^{1} \mathrm{D}{ }^{1} \mathrm{H}$ NMR spectra. The coplanar stacking of PcTS in solution (in $50 \mathrm{mM}$ phosphate buffer $\mathrm{pH} 6.8$ ) perturbates the diamagnetic shielding and trigger upfield chemical shifts in resonances of the protons located near the $\pi$ electron macroring, mostly the peripheral aromatic protons. At low concentrations of PcTS ( $50 \mu \mathrm{M}$, blue and $100 \mu \mathrm{M}$, red), both calculated and evaluated concentrations (from NMR spectra) are in accordance. At higher concentrations, the coplanar stacking results in a significant deviation between calculated and evaluated (i.e. $500 \mu \mathrm{M}$ versus $176 \mu \mathrm{M}$, green; and $1500 \mu \mathrm{M}$ versus $286 \mu \mathrm{M}$, pink). Measurements were conducted on BRUKER $400 \mathrm{MHz}$ spectrometer at $278 \mathrm{~K}$. 


\subsection{Phthalocyanine Tetrasulfonate}

\subsubsection{Biological applications}

PcTS is known for its diverse applications in biological systems and its antiamyloidogenic effect has been investigated in several studies. TSEs are group of rare neurodegenerative diseases that infect sheep (Scrapies), cattle (Bovine spongiform encephalopathy BSE), deer (Chronic wasting disease CWD) and humans (CreutzfeldtJakob disease CJD). These pathogeneses share a common event where the functional protease-sensitive prion protein ( $\mathrm{PrP}-\mathrm{sen})$ switches conformation to a partially proteaseresistant form (PrP-res). From a number of screened porphyrins and Phthalocyanines in scrapie-infected mouse neuroblastoma cell cultures, ${ }^{280} \mathrm{PcTS}$ exhibited in vivo prophylactic and therapeutic effects for its inhibition of PrP-res aggregates ${ }^{281}$ and substantially prolonged the lives of treated mice. ${ }^{282}$ A study was further carried to investigate whether the metal occupancy and the extent of sulfonation can strongly influence in vivo and in vitro potency of PrP-res inhibition. ${ }^{277}$ Although the compound's solubility increases dramatically with the number of sulfonates, the extent of PcTS sulfonation had little effect on $\mathrm{EC}_{50}$. PcTS and $\mathrm{Ni}(\mathrm{II}) \mathrm{PcTS}$ showed significantly delayed disease incubation times and together with $\mathrm{Fe}(\mathrm{III}) \mathrm{PcTS}$ were among the most potent (lowest $\mathrm{EC}_{50}$ ) in murine cells. The proposed mechanism of the anti-TSE activity suggests that cyclic tetrapyrroles bind PrP aggregates or stack sequentially onto a bound monomer via $\pi-\pi$ interactions of the aromatic system whereas electrostatic bonding interactions between negative $\mathrm{SO}_{3}{ }^{3-}$ and positive centers on target binding site contribute much less to the overall effect. A metal-sensitive property of cyclic tetrapyrroles may be relevant to inhibition and ability to oligomerize via various types of $\pi$ stacking. More recently, the interaction of free and metal-based PcTS with hamster PrP (90-232) has been further characterized by a handful of complementary assays. ${ }^{283}$ The high and low binding affinities of PcTS and AlPcTS, respectively, 


\subsection{Phthalocyanine Tetrasulfonate}

correspond to the high (PcTS) and low (AlPcTS) tendencies of self-stacking and are affected to a lower extent by the buffer ionic strength. It is, however, expected that changes in solvents and tissue microenvironments may well modify the equilibrium constants, kinetics of formation and dissociation of aggregates. ${ }^{28}$ Moreover, the binding is modulated by the aromatic residues with a stoichiometry of 4 to 5 PcTS moieties per PrP molecule. Indeed, this oligomerization process has been demonstrated to be essential for the inhibitory activity of PcTS on protein aggregation. Importantly, PcTS suppresses the formation of AS amyloid. ${ }^{284}$ Using NMR spectroscopy, it was shown that PcTS inhibits $\alpha \mathrm{S}$ filament assembly and induces the formation of non-toxic aggregates mediated by specific binding to aromatic residues at the N-terminus (Y39). ${ }^{285}$ This study provided crucial evidences that PcTS diminishes the amount of aggregated $\alpha \mathrm{S}$ in a dose dependent fashion and converts them into smaller amorphous nonfibrillar species that are non-toxic, have reduced $\beta$-sheet signature (CD spectroscopy), and are sensitive to dissolution by mild detergent Sarkosyl. Taking advantage of its chelating and molecular self-assembly properties, PcTS forms a complex with the redox-active metals that induce toxic A $\beta_{40}$ oligomeric species and results in an amyloid fibrillar meshwork. ${ }^{235}$ Surprisingly, there are few studies on PcTS as a possible candidate in disassembling Tau protein filaments but with no further structural or mechanistic basis. Recently, we revealed the mechanism behind the interaction between PcTS and full-length human Tau protein. ${ }^{60}$ By combining NMR spectroscopy, electron paramagnetic resonance spectroscopy (EPR) and Smallangle X-ray scattering (SAXS) together with other biophysical techniques, we demonstrated that PcTS targets tyrosine residues of monomeric hTau40 and converts the protein into soluble oligomers that contain a dynamic noncooperatively stabilized core. The experimental details and extensive discussion of results are presented in Chapter 2. 


\subsection{Methylene Blue}

$\mathrm{MB}$ has received considerable attention as a potential drug candidate for targeting $\mathrm{AD}$; not only for its inhibition of Tau aggregation but also for its regulation of $\mathrm{A} \beta$ fibril formation. Non-neuroleptic $\mathrm{MB}$ penetrates the $\mathrm{BBB}$ and its dimethyl derivatives have enhanced properties for aggregation. ${ }^{286}$ Together with other beneficial pharmacokinetics, the determining factors for an optimal mode of action depends on the oxidation-reduction behaviour, planarity and aromaticity of the system.

\subsubsection{Physical, Chemical and Biochemical Properties}

As previously mentioned, $\mathrm{MB}$ is a derivative of the hetero tricyclic phenothiazine exhibiting a cationic thiazine ring and two dimethylamino functional groups $\left(\mathrm{NMe}_{2}\right)$ on the outer benzene rings at positions R3 and R7 (Table 3). Molecules similar in structure and physical properties to the parent compound MB include Azure $A\left(\mathrm{R} 3=\mathrm{NMe}_{2}, \mathrm{R} 7=\mathrm{NH}_{2}\right)$, Azure $B\left(\mathrm{R} 3=\mathrm{NMe}_{2}, \mathrm{R} 7=\mathrm{NHMe}\right)$, Azure $C\left(\mathrm{R} 3=\mathrm{NHMe}, \mathrm{R} 7=\mathrm{NH}_{2}\right)$ and Thionin $(\mathrm{R} 3=$ $\mathrm{NH}_{2}, \mathrm{R} 7=\mathrm{NH}_{2}$ ). The cationic thiazine ring of $\mathrm{MB}$ and the azures establishes the planarity and aromaticity of the system and through oxidation-reduction reactions these compounds convert to the neutral nonaromatic Leuco forms (Figure 13). MB and its derivatives absorb around $670 \mathrm{~nm}$ and display photosensitizing properties, whereupon the presence of oxygen and light, they generate a singlet oxygen ${ }^{287}$ which is substantially vital in photodynamic therapy and production of organic peroxides.

Similar to PcTS and other organic dyes, MB forms dimers and higher order aggregates in a concentration dependent manner. Importantly, the response of MB in most biological systems is characterized by a hormetic behavior. Hormesis defines a drug with 
opposing effects in low and in high doses. In low $\mu \mathrm{g}$ ranges, $\mathrm{MB}$ is neuroprotective, acting as an electron donor and an antioxidant in mitochondria; while at higher doses it withdraws electrons away from the mitochondrial transport chain complexes and alters the activity of the associated enzyme, cytochrome oxidase. ${ }^{288}$ A study using rat brain homogenates proposed a mechanism by which the autoxidizable property of MB provokes the hormetic biological effects on cytochrome oxidase. The iron center of this enzyme increases its activity by oxidizing low concentrations of LMB to MB. At higher MB doses, the reversible equilibrium between both species (LMB and $\mathrm{MB}$ ) is lost. The activity of the enzyme then decreases as excess MB withdraws electrons from the transport chain complexes. This hormetic dose-response has been also reported in the treatment of Methemoglobinemia. At a low concentration of MB, the targeted NADP-methemoglobin reductase converts methemoglobin to hemoglobin. ${ }^{289}$ However, at higher concentrations and stronger oxidizing capacity, the effect is reversed and MB causes itself methemoglobinemia. ${ }^{290}$ This proves that MB does not exhibit a pharmacological dosedependent profile but rather its effective concentration would determine its role as an electron donor or acceptor. 
<smiles>CNc1ccc2nc3ccc(N(C)C)cc3[s+]c2c1</smiles>

Azure B (oxidized form)<smiles>CNc1ccc2c(c1)Sc1cc(N(C)C)ccc1N2</smiles>

Azure B (reduced form)

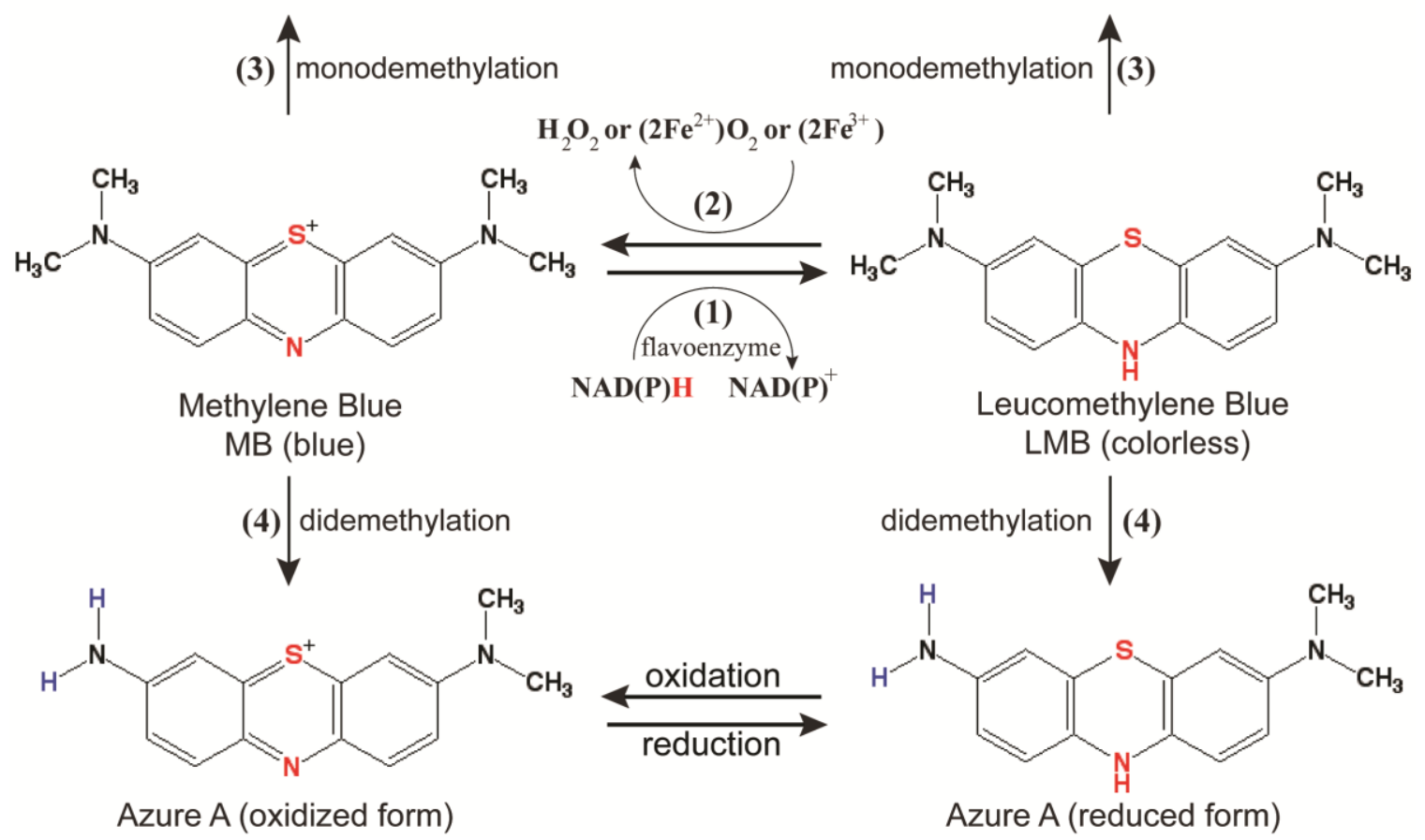

Figure 13: Reduction-Oxidation Interplay of Phenothiazines.

The reduction of MB yields leucoMB (1) and is regenerated in oxidizing conditions (2). Mono- and di- $N$ demethylation yield azure B (3) and azure A (4), respectively. Adapted from ${ }^{291}$.

\subsubsection{Biological applications}

During the international conference on Alzheimer's Disease held in 2008, a major breakthrough in the treatment of $\mathrm{AD}$ was announced from clinical trials sponsored by TauRx Therapeutics. ${ }^{292}$ Based on the designed formulation, RemberTM, containing an optimized form of $\mathrm{MB}$, the report presented evidence that this drug dissolves the tangle filaments isolated from AD brains, and disrupts abnormal Tau aggregation in cell models. 
Upon oral administration of MB to 321 participants in a phase II clinical trial over 84 weeks, patients with mild to moderate cognitive impairment exhibited $81 \%$ reduction of cognitive decline rate at week $50 .{ }^{239}$ These findings triggered a wave of scientific supports and arguments; as well as a handful of strategic measurements over the years that followed. From extensive pharmacokinetic studies performed on rat models, MB distribution among organs was found to be dependent on the form of administration, but its permeation of the BBB was irrespective of the route. ${ }^{293}$ Along its biochemical pathways, MB undergoes an in vivo redox cycle where it is reduced by NADPH into the leucoMB (LMB). In the presence of molecular oxygen or Fe(III)-containing compounds, LMB is oxidized to regenerate $\mathrm{MB}$ (Figure 13). In parallel, the mono- and di-N-demethylation of $\mathrm{MB}$ respectively yield azure B and azure A; two metabolites with established pharmacological effects. ${ }^{294}$ Furthermore, the oxidized forms of MB derivatives exhibiting one or more amino hydrogens $\left(\mathrm{NHMe}\right.$ or $\mathrm{NH}_{2}$ ) can potentially form a neutral quinoneimine upon deprotonation (Figure 14). This species readily diffuses through membranes in contrast to the charged MB.

A large number of hypotheses were proposed to postulate the protective role of $\mathrm{MB}$ in neurodegeneration. One approach is based on cycling between the oxidized and reduced forms of MB. ${ }^{295}$ Other studies compare the potential inhibition of Tau aggregation by MB and azure B with a therapeutic superiority of the latter. A third suggestion involves the Hsp70 chaperone where the MB-driven inhibition of Hsp70 ATPase modulates the degradation of toxic polyglutamine protein and increases its accumulation in the cell. ${ }^{296}$ With the same Hsp70 inhibition scheme, the level of Tau protein is selectively decreased and consequently provokes cell protection from toxic accumulations. ${ }^{297}$ Lining up the recently published failure of MB in zebra fish models of Tauopathy ${ }^{298}$ and its interpretation 
with a biphasic dose-response relationship, it is still unclear whether the therapeutic behaviour of MB corresponds to its inhibitory action on Tau aggregation, its antioxidant activity by interaction with electron transport chain of mitochondria, or its binding and modulation of partner proteins. Nevertheless, MB has recently stepped into a phase III clinical trial. 299

The fundamental link between $\mathrm{MB}$ and azure $\mathrm{B}$ had to be further clarified to determine whether the monodemethylation favoured with several in vitro and in vivo conditions, confirms that $\mathrm{MB}$ is the prodrug of the active agent azure $\mathrm{B}$. $\mathrm{MB}$ is one of the first compounds identified that blocked Tau-Tau interaction. To access additional insights on the efficacy of MB and the interplay of both azure A and azure B, we were interested in identifying their possible interactions with Tau protein at molecular levels. ${ }^{291}$ Our findings suggest that the mode of action of these phenothiazines lies behind the interplay in the reduction/oxidation potential of their thiazine ring and the native cysteine residues of Tau. Using NMR spectroscopy and other biophysical techniques, we demonstrated that these compounds specifically modify the cysteines and retain Tau in a monomeric disordered conformation. Chapter 3 presents the experimental details and an extensive discussion of the results. As previously mentioned (part 1.4.2), the oxidation of Tau at its cysteines (C291, C322) is a posttranslational modification that has an impact on aggregation driven by a disulfide bond. This will be comprehensively discussed furthermore in part 1.8.3.

At the same time, MB has been widely investigated for its inhibition of aggregating proteins deposited in other neurodegenerative diseases. In TSEs, MB binding to hydrophobic and charged residues provoked a diminished rate of PrP-res oligomerization and limited fibrillar formation. ${ }^{300}$ On the other hand, $M B$ targets $A \beta_{42}$ differently by inhibiting monomeric conversion into oligomers but enhancing fibril formation; hence 
suggesting that oligomerization and fibrillization of $A \beta_{42}$ follow different pathways. ${ }^{301}$ All in all, several modes for the protective role of $\mathrm{MB}$ in neurodegeneration have been postulated, and although the mechanisms differ widely but many of them are reinforced with experimental and clinical observations.

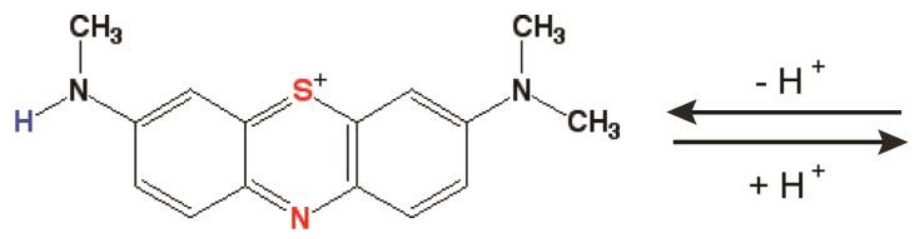

Azure B (oxidized form)

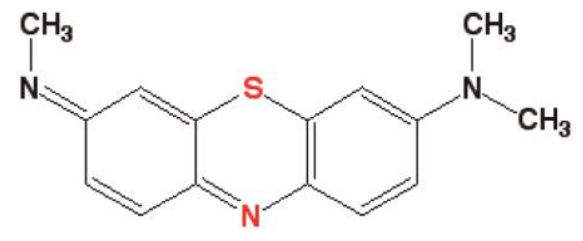

Quinoneimine

Figure 14: Conversion of Azure B to Quinoneimine.

Oxidized phenothiazines azure $\mathrm{A}, \mathrm{B}$ and $\mathrm{C}$ can potentially undergo deprotonation to yield a neutral quinoneimine.

\subsubsection{The Impact of MB on Cysteine Oxidation}

The nucleophilic sulfhydryl group of a cysteine amino acid is prone to chemical modification. ${ }^{302}$ The sulfur character undergoes several distinct redox pathways and facilitates regulatory roles in electron donation, hydride-transfer reactions, and free radical reactions. ${ }^{303}$ The most dominant chemical oxidation is the disulfide bridge formation where it enhances structural stability in some cases, and initiates protein aggregation in other cases. Sickle-cell hemoglobin, $A \beta$, and Tau were among the identified proteins that undergo fibrillization following a nucleus-driven mechanism, known as seeding or nucleation. ${ }^{304}$ Therefore, screening compounds that interfere with the disulfide bond formation is a possible therapeutic intervention for inhibiting Tau assemblies. 
The two native cysteine residues of Tau (C291, C322) exist in the repeat regions $\mathrm{R} 2$ and $\mathrm{R} 3$, hence, $3 \mathrm{R}$ and $4 \mathrm{R}$ isoforms contain respectively one or two cysteines. Accordingly, 4R isoforms exhibit an intramolecular disulfide (S-S) bond between C291 and $\mathrm{C} 322$, whereas the sole cysteine in 3R isoforms (C322) favors intermolecular disulfide bonding and consequently formation of dimers ${ }^{305}$ and higher aggregates. ${ }^{58,176}$ However, 4R isoforms are not completely resistant to aggregation but they indeed exhibit both intra and intermolecular S-S bonds and consequently a mixture of monomers, dimers and higher aggregates. $^{306}$

Lining up the suggested involvement of cysteine-induced dimers in the pathological cascade (Figure 10) and the potential of phenothiazines in disrupting Tau aggregates, we studied the oxidation-reduction behaviour of $\mathrm{MB}$ and its metabolites azure A and azure $\mathrm{B}$ for determining factors of Tau aggregation inhibition. ${ }^{291}$ Our study showed that these organic molecules inhibit aggregation of Tau via oxidation of the native cysteine residues to sulfenic, sulfinic and sulfonic acids. Cysteine-oxidation keeps monomeric Tau in an aggregation-incompetent disordered state avoiding the formation of toxic Tau aggregation intermediates. Moreover, our aggregation assays performed on the 3R construct, K19, demonstrated that disulfide bond formation is diminished in the presence of $\mathrm{MB}$ and aggregation of K19 is not promoted. We also showed that demethylation of MB provides new modes of interaction with Tau. Similar to MB, the metabolites azure A and azure B oxidize Tau's cysteines, but also interacts with aromatic residues, indicating that in vivo where $\mathrm{MB}$ can be modified to azure $\mathrm{A}$ and azure $\mathrm{B}$, additional modes of Tau aggregation modulation are possible. The experimental details and extensive discussion of the results are presented in Chapter 3. 


\subsubsection{MB and Protein Sulfenic Acids}

Despite the fact that disulfide bond formation is the most prevailing chemical modification of cysteines that occurs under different conditions, thiols undergo a wide range of oxidation states from +6 to -2 . Peroxidation governs the formation of cysteinesulfenic acid (Cys-SOH), a species that play an essential role in redox control of many protein functions, such as enzymatic catalysis and cell signaling. Protein-SOHs are in the center of both regulatory and pathological events. The Cys-SOH is moderately stable but in the presence of a free thiol, it can form disulfide bonding, or progressively proceed to higher oxidation orders to yield cysteine-sulfinic acid $\left(\mathrm{Cys}-\mathrm{SO}_{2} \mathrm{H}\right)$ and cysteine-sulfonic acid $\left(\mathrm{Cys}-\mathrm{SO}_{3} \mathrm{H}\right) \cdot{ }^{307}$ The nature of these transient species and their high reactivity both increase the challenge in detecting, isolating and quantifying them. Nevertheless, the different forms of oxidized cysteines have been detected in several signal transduction cascades and regulatory proteins including protein tyrosine phosphatases, ${ }^{308}$ peroxiredoxins, ${ }^{309}$ glyceraldehyde-3-phosphate dehydrogenase, ${ }^{310}$ cysteine protease papain, ${ }^{311}$ and human serum albumin. ${ }^{312}$

Yet another significant involvement of MB-mediated oxidation was observed with the molecular chaperone family of heat shock proteins 70 (Hsp70). ${ }^{296}$ These proteins bind ATP through their N-terminal domain and trigger conformational changes in their $\mathrm{C}$ terminal domain. Consequently, this enhances the affinity to interact with their unfolded substrates and thereby protecting them against aggregation. (Part 1.9 will discuss the Hsp family in more details). Hsp70s were implicated in reducing Tau accumulation and have been linked to redox signaling based on the reactivity of the cysteine residues. ${ }^{313}$ The two proteins, Hsc70 and Hsp72, have similar sequences and exhibit four or five cysteine residues, respectively. The additional cysteine of Hsp72, Cys306, has been associated to 
the ATPase activity. The MB-mediated oxidation of Cys306 contributed in the loss of ATP binding. Moreover, upon treatment of HeLaC3 cells with MB, the designed Hsp72/C306S mutant (resistant to MB oxidation) blocked the clearance of Tau whereas the Hsp72/C306D mutant (mimicking oxidation) reduced Tau levels in these cells. Of all cysteines, MB oxidized Hsp72 at Cys306 to Cys-SOH and had no effect on any of the cysteines of Hsc70. This modification blocked Hsp72 from binding ATP and hence compromised its function in regulating levels of Tau. Keeping in mind that Hsp72 is upregulated during stress, this provided its direct participation in stress-related redox signaling and oxidative stress. 


\subsection{Heat Shock Protein}

The collection of Heat shock proteins (Hsp) include members of functionally related proteins that are highly expressed when exposed to high temperatures, $\mathrm{pH}$ alteration, or oxygen deprivation. These proteins are abundant in all living organisms and constitute essential factors for cell growth and viability. Hsps are molecular chaperones that are mostly involved in folding and refolding mechanisms of denaturated proteins. ${ }^{149}$ They prevent protein aggregation by interacting to exposed hydrophobic surfaces of aggregation-prone polypeptides. Other functions include protein assembly, translocation and degradation of misfolded aggregates. Hsps are further classified into major families based on their related functions and molecular masses. These classes comprise Hsp70, Hsp40, Hsp60, Hsp90, Hsp100 and other small heat shock proteins, sHsps (Figure 15). Within a sophisticated molecular network, these proteins function cooperatively to maintain a stable cellular homeostasis.

Proteins of the Hsp70 family consist of two conserved functional domains, nucleotide binding domain (NBD) and substrate binding domain (SBD) both connected by a flexible linker. This family mainly supports refolding of misfolded and aggregated proteins but also import unfolded species to major cellular compartments (mitochondria, endoplasmic reticulum) for folding or degradation purposes.

The specificity of Hsp70 interaction is determined by members of the Hsp40

family (known as $J$-proteins). The four $\alpha$-helical domains of Hsp40 stimulate the activity of ATPase $\mathrm{A}^{314}$ and confiscate denaturated substrates by controlling their interaction with Hsp70. ${ }^{315}$ The SBD of the Hsp70 then regulates substrate binding via its helical regions. Selective nucleotide factors affect the replacement of ATP by ADP, and consequently the dissociation of the complex. Free Hsp70 reorganizes for repeating cycles to ensure proper 
folding. Hence, the co-chaperones Hsp40-Hsp70 constitute the overall molecular chaperone machine that is responsible for early stages of protein folding and refolding.

Following substrate binding of Hsp70, another class of proteins, Hsp90, has been reported for further interactions. ${ }^{316}$ But unlike other chaperones, Hsp90 proteins bind their targets at an advanced stage of folding. Hsp90 plays a major role in the folding scheme of essential client proteins like protein kinases and transcription factors. Both N- and Cdomains of Hsp90 interact with the substrate, however the N-terminus accommodates the ATP binding domain that recognizes co-chaperones and client proteins. The $\mathrm{C}$-terminal residues of Hsp90 assembles the homodimer with the ATP-bound state adopting a circular structure.

Other classes of the Hsp proteins are equally important. Hsp60 is a family of chaperonins that facilitates an ATP-mediated process responsible of folding multimeric proteins. Hsp100, on the other hand, solubilizes misfolded proteins and clears irreversible protein aggregates. After binding to ATP, Hsp100 accumulates into hexameric ring-shaped complexes that favor proteasomal degradation. One class of the Hsp family includes proteins with smaller molecular masses ranging between 12 and $43 \mathrm{kDa}$. These sHsp proteins adapt a conserved $\alpha$-crystalline domain. Oligomeric species of sHsps assemble into large surface areas to accommodate the aggregates and transfer non-native proteins to other Hsp machinery for proper folding.

Several Hsp molecular chaperones are prominent in proteasomal degradation of highly phosphorylated Tau. ${ }^{115,209,210}$. 


\subsection{Heat Shock Protein}

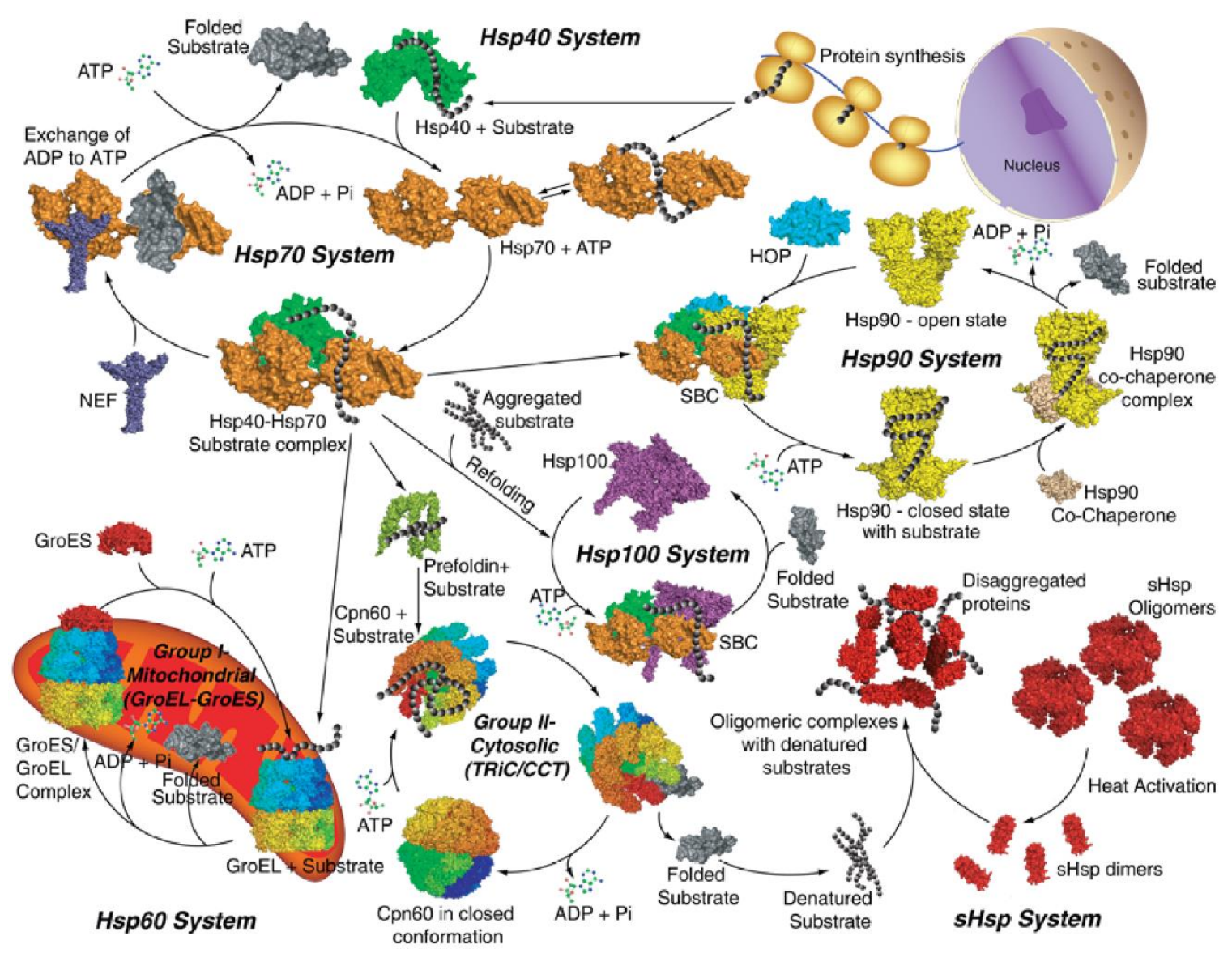

Figure 15: The Hierarchy of Heat Shock Proteins.

Heat shock proteins are functionally related molecular chaperones that are involved in folding mechanisms of denaturated proteins. They classified into major families based on their related functions and molecular masses. These classes comprise Hsp70, Hsp40, Hsp60, Hsp90, Hsp100 and other small heat shock proteins. Adapted from ${ }^{317}$.

\subsubsection{Molecular Chaperones and Tau Protein}

The major functions behind Hsp70 clearance of Tau aggregates are associated to ATP hydrolysis and peptide binding. The N-terminal ATPase domain triggers conformational changes of the NBD and SBD domains upon ATP binding. The hydrolysis of the ATP-bound form to the ADP-bound arrangement increases the affinity for substrate 
binding. The SBD then switches from an open to a closed conformation and activates its binding pockets. Favored by a high affinity for neutral and hydrophobic residues, the Cterminal SBD binds its target. In parallel, Hsp40 (J-proteins) catalyzes the conversion of ATP to ADP, stabilizes the substrate-bound form, and further recruits other substrates to close proximity of active Hsp70. Once proper folding occurs, selective nucleotide factors affect the replacement of ATP by ADP, hence remodeling the ATP-bound form. The SBDopen conformation then releases the substrate and consequently dissociates the complex. Unbound Hsp70 undergoes multiple folding/re-folding cycles. The Hsp40-Hsp70 chaperone machine ensures proper protein folding/refolding and prevention of misfolded species. Remarkably, Hsp70 inhibitory mode exhibits higher preferences to oligomeric Tau aggregates over fibrillar. ${ }^{318}$ The affinity towards 3 repeat isoforms was found to be higher. ${ }^{319}$

On the other hand, the cooperative functioning of Hsp70 and Hsp90 have been widely reported. ${ }^{320}$ Hsp70 is monomeric and contains a single ATP-binding pocket that can be activated by Hsp40 whereas Hsp90 occurs as a homodimer accommodating four ATPbinding pocket that are independent of Hsp40 activation. ${ }^{321}$ The levels of Hsp70 and Hsp90 are crucial in determining clearance or preservation of Tau aggregates. ${ }^{322}$ The high ATP consumption of both proteins, specifically by Hsp90, triggered the concept of modulating their ATPase activities through pharmacological intervention and genetic manipulation.

Among the Hsp70 family, protein Hsp72 and heat shock cognate 70 (Hsc70) protein were highly relevant in tau degradation. ${ }^{323}$ In mammalian systems, Hsc70 is constitutively expressed while Hsp72 expression is stress-induced. Although the two proteins exhibit more than $80 \%$ resemblance in their primary sequences with minor variances at the C-terminal, both proteins influence Tau conformation and clearance 
kinetics differently. Constitutive Hsc70 slowed Tau clearance while inducible Hsp72 accelerated it. ${ }^{324}$ In addition to these opposing effects, Hsp72 exhibited higher affinity for Tau than Hsc70 as accessed by NMR spectroscopy. These findings were directly associated to the minor sequence differences at the C-terminus of both proteins and supported therapeutic relevance in promoting Hsp72 and inhibiting Hsc70. Chapter 4 presents these findings together with experimental details and extensive discussion of the results. 


\subsection{NMR Spectroscopy}

NMR spectroscopy is an indispensable tool in structural elucidation of small organic molecules and large biomolecules. The classical NMR parameters such as chemical shifts, scalar couplings and nuclear Overhauser enhancement all provide valuable information about the environment of nuclear spins. Tremendous number of protein structures of challenging systems have been solved by the aid of advanced NMR approaches. NMR-directed studies of protein-ligand interactions have augmented considerably the understanding of wide range of biological processes from neurotransmitter and drug binding to enzyme-substrate interactions. NMR-based FBDD quantitatively improved SAR approaches and expanded the confidence and the number of screened libraries.

Table 4: Common NMR Parameters.

\begin{tabular}{lcll} 
NMR Parameter & Symbol & NMR Parameter & Symbol \\
\hline Magnetic moment & $\mu$ & Gyromagnetic ratio & $\gamma$ \\
Angular momentum & $\mathrm{I}$ & External magnetic field & $\mathrm{B}$ \\
\hline Larmor frequency & $\omega_{0}$ & Chemical shift & $\delta$ \\
\hline Scalar coupling & $J$ & Rotational correlation time & $\tau_{\mathrm{C}}$ \\
\hline Longitudinal relaxation rate & $R_{1}$ & Transverse relaxation rate & $R_{2}$ \\
\hline Lateral Diffusion & $D$ & Diffusion time & $\Delta$
\end{tabular}


Each type of nuclear proton is characterized by a magnetic moment $\boldsymbol{\mu}$ relating the spin angular momentum $\hat{I}$ to its gyromagnetic ratio $\boldsymbol{\gamma}$ and Planck's constant $\boldsymbol{h}$ :

$$
\boldsymbol{\mu}=\frac{\gamma \cdot h \cdot \hat{I}}{2 \pi}
$$

10.1

When positioned in an external magnetic field $\boldsymbol{B}$, the magnetic moment $\boldsymbol{\mu}$ orients with the static field along the z-axis, $\boldsymbol{B}_{\boldsymbol{0}}$, and the nuclear spins split into quantized energy levels. In the case of a nucleus with spin $\hat{\mathrm{I}}=\frac{\mathbf{1}}{\mathbf{2}}$ such as ${ }^{1} \mathrm{H}$ or ${ }^{13} \mathrm{C}$, the two occurring states are:

$$
\begin{aligned}
& E(\alpha)=-\frac{1}{2} \gamma \cdot \hbar \cdot B_{0} \\
& E(\beta)=+\frac{1}{2} \gamma \cdot \hbar \cdot B_{0}
\end{aligned}
$$

where $\hbar=\frac{\boldsymbol{h}}{\mathbf{2}}$. Transitions between these energy levels can be induced upon irradiation with a radiofrequency $h \boldsymbol{v}$ corresponding to the energy difference between two states:

$$
\Delta E=\gamma \cdot \hbar \cdot B_{0}=h v
$$

The Larmor frequency $\boldsymbol{\omega}_{0}$ of a nuclear spin only depends on the magnetic field and its gyromagnetic ratio:

$$
\omega_{0}=\gamma \cdot B_{0}
$$




\subsubsection{NMR Chemical Shifts}

The observed nuclear larmor frequency depends on the local chemical environment and defines the chemical shift $\boldsymbol{\delta}$ as the difference between this frequency and a reference frequency:

$$
\delta(p p m)=10^{6} .\left(\frac{v-v_{0}}{v_{0}}\right)
$$

In peptides and proteins, the backbone conformation and the associated chemical shifts ${ }^{1} \mathrm{H}_{\alpha},{ }^{1} \mathrm{H}_{\mathrm{N}}$ and ${ }^{13} \mathrm{C}_{\alpha}$ are directly correlated. ${ }^{325}$ Ring currents of aromatic residues affect $\boldsymbol{\delta} \mathrm{H}_{\alpha}$ values whereas hydrogen bonding and dihedral angles mainly influence $\boldsymbol{\delta} \mathrm{H}_{\mathrm{N}}$ values. ${ }^{326}$ Moreover, the chemical shifts ${ }^{1} \mathrm{H}_{\alpha},{ }^{13} \mathrm{C}_{\alpha},{ }^{13} \mathrm{C}_{\beta}$, and ${ }^{13} \mathrm{CO}$ are sensitive probes to secondary structure. The secondary chemical shift $\boldsymbol{\Delta} \boldsymbol{\delta} \boldsymbol{C}$ identifies these elements. Relative to random coil values, ${ }^{327}$ experimental $\boldsymbol{\delta} \mathrm{C}_{\alpha}$ and $\boldsymbol{\delta} \mathrm{CO}$ shift upfield in $\beta$-sheets $(\boldsymbol{\Delta} \boldsymbol{\delta} \boldsymbol{C}<\mathbf{0})$ and downfield in $\alpha$-helices $(\boldsymbol{\Delta} \boldsymbol{\delta} \boldsymbol{C}>\mathbf{0})$.

$$
\Delta \delta C=\delta C_{e x p}-\delta C_{c o i l}
$$

The secondary chemical shift $\boldsymbol{\Delta} \boldsymbol{\delta} \boldsymbol{C}$ identified local structural propensities in different IDPs such as prion, ${ }^{328} \alpha \mathrm{S},{ }^{329} \beta \mathrm{S},{ }^{330}$ and Tau. ${ }^{86}$ 


\subsubsection{Heteronuclear Single Quantum Coherence}

The standard 2D NMR measurement is the heteronuclear single quantum coherence (HSQC) experiment. This pulse sequence records one-bond correlation spectra between ${ }^{1} \mathrm{H}$ and ${ }^{13} \mathrm{C}$ or ${ }^{15} \mathrm{~N}$. The sensitivity of NMR is governed by the natural abundance of the active nucleus and its gyromagnetic ratio. Since the proton satisfies both requirements, the transfer of magnetization from proton to other nuclei allows the measurement of heteronuclear NMR experiments with high sensitivity. The main scheme involves a proton $\left({ }^{1} \mathrm{H}\right)$ excitation, then polarization transfer to the selected heteronucleus $\left({ }^{13} \mathrm{C}\right.$ or $\left.{ }^{15} \mathrm{~N}\right)$ and finally a polarization back transfer for detection on protons. A common building block for this purpose is the Insensitive nuclei enhancement through polarization transfer (INEPT). The pulse sequence of a standard HSQC experiment combines two INEPT magnetization transfers, the first between periods $\mathrm{A}$ and $\mathrm{B}$ and the second between $\mathrm{D}$ and $\mathrm{E}$ (Figure 14). The first $90^{\circ}$ pulse flips the spin $\boldsymbol{I}$ from equilibrium $\left(\hat{\mathrm{I}}_{\mathbf{z}}\right)$ to a transverse magnetization $\left(\hat{\mathrm{I}}_{\boldsymbol{y}}\right.$ ). During period $A$ (known as spin echo), the $180^{\circ}$ pulse on both $\boldsymbol{I}$ and $\boldsymbol{S}$ spins refocuses the chemical shift modulation but allows coupling to evolve $\left(2 \hat{\mathrm{I}}_{\boldsymbol{x}} \hat{\mathrm{S}}_{\mathbf{z}}\right)$. The two $90^{\circ}$ pulses at period $B$ transfer the coherence to the directly-attached heteronucleus $\left(2 \hat{\mathrm{I}}_{\boldsymbol{x}} \hat{\mathrm{S}}_{\boldsymbol{z}} \rightarrow 2 \hat{\mathrm{I}}_{z} \hat{\mathrm{S}}_{\boldsymbol{y}}\right.$ ) During period $C$, $\mathrm{t}_{1}$ evolution takes place but the central $180^{\circ}$ pulse on spin $\boldsymbol{I}$ refocuses the evolution of the heteronuclear coupling $\boldsymbol{J}_{\boldsymbol{I S}}\left(2 \hat{\mathrm{I}}_{\mathbf{z}} \hat{\mathrm{S}}_{\boldsymbol{y}}\right)$. Simultaneous $90^{\circ}$ pulses at period $D$ transfer the anti-phase magnetization back to spin $\boldsymbol{I}$ $\left(2 \hat{\mathrm{I}}_{z} \hat{\mathrm{S}}_{\boldsymbol{y}} \rightarrow-2 \hat{\mathrm{I}}_{\boldsymbol{y}} \hat{\mathrm{S}}_{\boldsymbol{z}}\right)$. The second spin echo during period $E$ transforms the anti-phase component back to the in-phase magnetization $\left(-2 \hat{\mathrm{I}}_{y} \hat{\mathrm{S}}_{z} \rightarrow \hat{\mathrm{I}}_{x}\right)$. The signal is finally detected in the proton dimension. A modification to the standard pulse sequence is the constant time (ct) HSQC (Figure 14), in which an additional element is inserted between the two INEPT sequences so that the heteronuclear coherence evolves during a constant 
time period $T$. The proper adjustment of $T$ avoids splitting of ${ }^{13} \mathrm{C}^{13} \mathrm{C}$ couplings in the ${ }^{13} \mathrm{C}$ dimension.
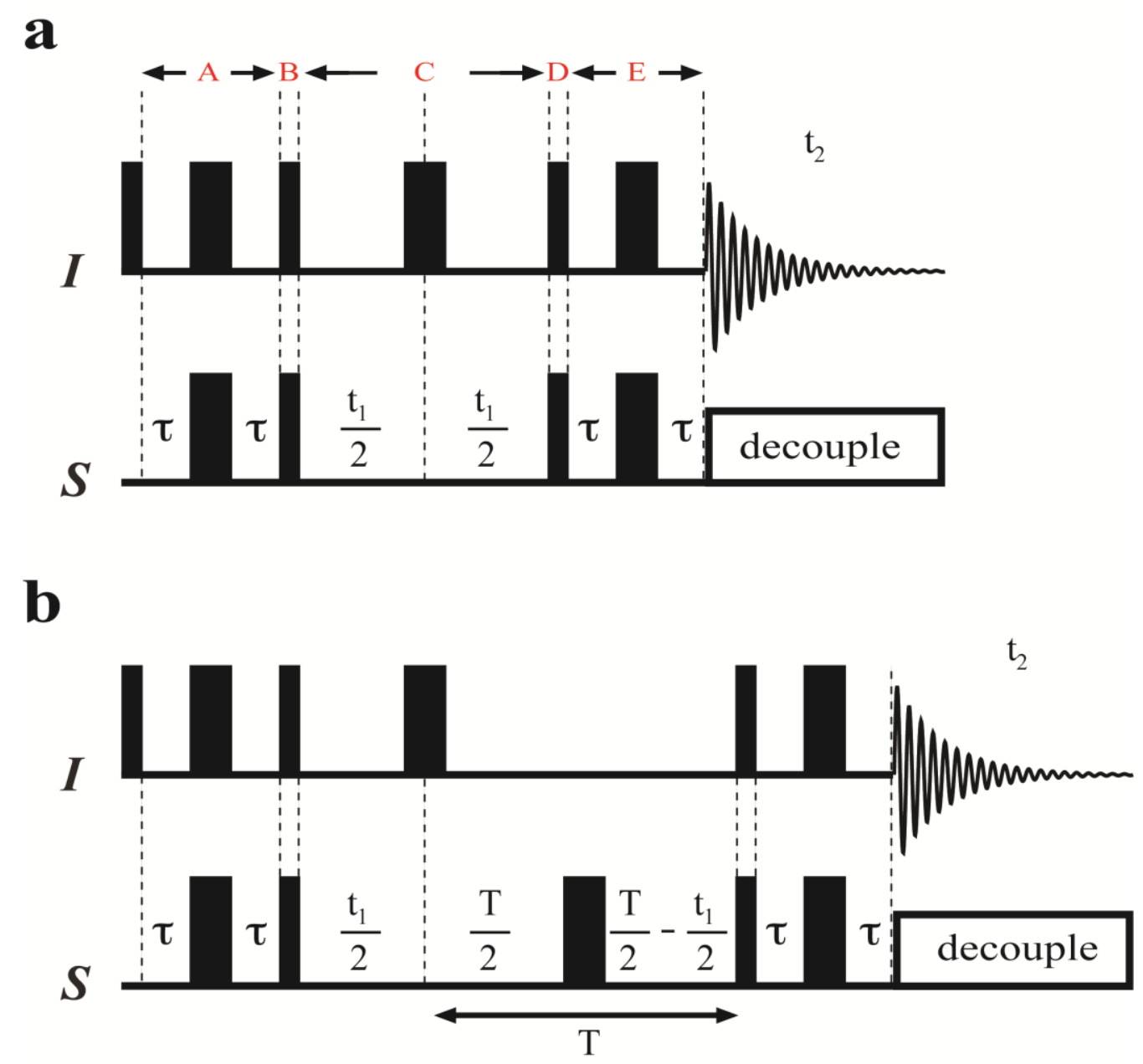

Figure 16: Pulse Sequences for Basic and Constant-time HSQC.

(a) The pulse sequence of a standard HSQC experiment involves two INEPT magnetization transfers for proton excitation, polarization transfer to the selected heteronucleus, and back transfer for detection on protons. $\left[90^{0}\right.$ pulse $\rightarrow\left(\hat{\mathrm{I}}_{\boldsymbol{y}}\right) ; 180^{0}$ pulse $\rightarrow\left(2 \hat{\mathrm{I}}_{\boldsymbol{x}} \hat{\mathrm{S}}_{z}\right) ; 90^{0}$ pulses $\rightarrow\left(2 \hat{\mathrm{I}}_{\boldsymbol{x}} \hat{\mathrm{S}}_{\boldsymbol{z}} \rightarrow 2 \hat{\mathrm{I}}_{z} \hat{\mathrm{S}}_{\boldsymbol{y}}\right) ; 180^{0}$ pulse $\rightarrow\left(2 \hat{\mathrm{I}}_{z} \hat{\mathrm{S}}_{\boldsymbol{y}}\right) ; 90^{0}$ pulses $\left.\rightarrow\left(2 \hat{\mathrm{I}}_{z} \hat{\mathrm{S}}_{\boldsymbol{y}} \rightarrow-2 \hat{\mathrm{I}}_{\boldsymbol{y}} \hat{\mathrm{S}}_{z}\right) \rightarrow\left(-2 \hat{\mathrm{I}}_{\boldsymbol{y}} \hat{\mathrm{S}}_{z} \rightarrow \hat{\mathrm{I}}_{\boldsymbol{x}}\right)\right]$. (b) The constant time (ct) HSQC incorporates an additional element for the evolution of the heteronuclear coherence during a constant time period $T$ to avoid splitting of ${ }^{13} \mathrm{C}-{ }^{13} \mathrm{C}$ couplings. Adapted from ${ }^{331,332}$. 


\subsection{NMR Spectroscopy}

The successful use of $2 \mathrm{D}{ }^{1} \mathrm{H}_{-}{ }^{15} \mathrm{~N}$ HSQC is based on the large dispersion of backbone ${ }^{15} \mathrm{~N}$ amide and ${ }^{13} \mathrm{C}$ carbonyl chemical shifts and their dependency on the local environment. In a folded protein, residues on the surface are present in a hydrophilic surrounding in contrast to the ones buried in the hydrophobic core. Both groups have distinct environments, and hence their chemical shifts are very different. This leads to the obvious spreading of chemical shifts (Figure 15). On the other hand, residues in an IDP share similar and averaged chemical environments where the resulting chemical shifts are primarily affected by their own side chain and their neighboring residues. Each residue type exhibits a corresponding random coil value, where ${ }^{15} \mathrm{~N}$ random coil values are better dispersed (100-135 ppm) than the ${ }^{1} \mathrm{H}$ values $(6.5-9.5 \mathrm{ppm}) .{ }^{333}$ The chemical shifts of residues in IDPs are indeed close to these random coil values and hence the dispersion is small. 


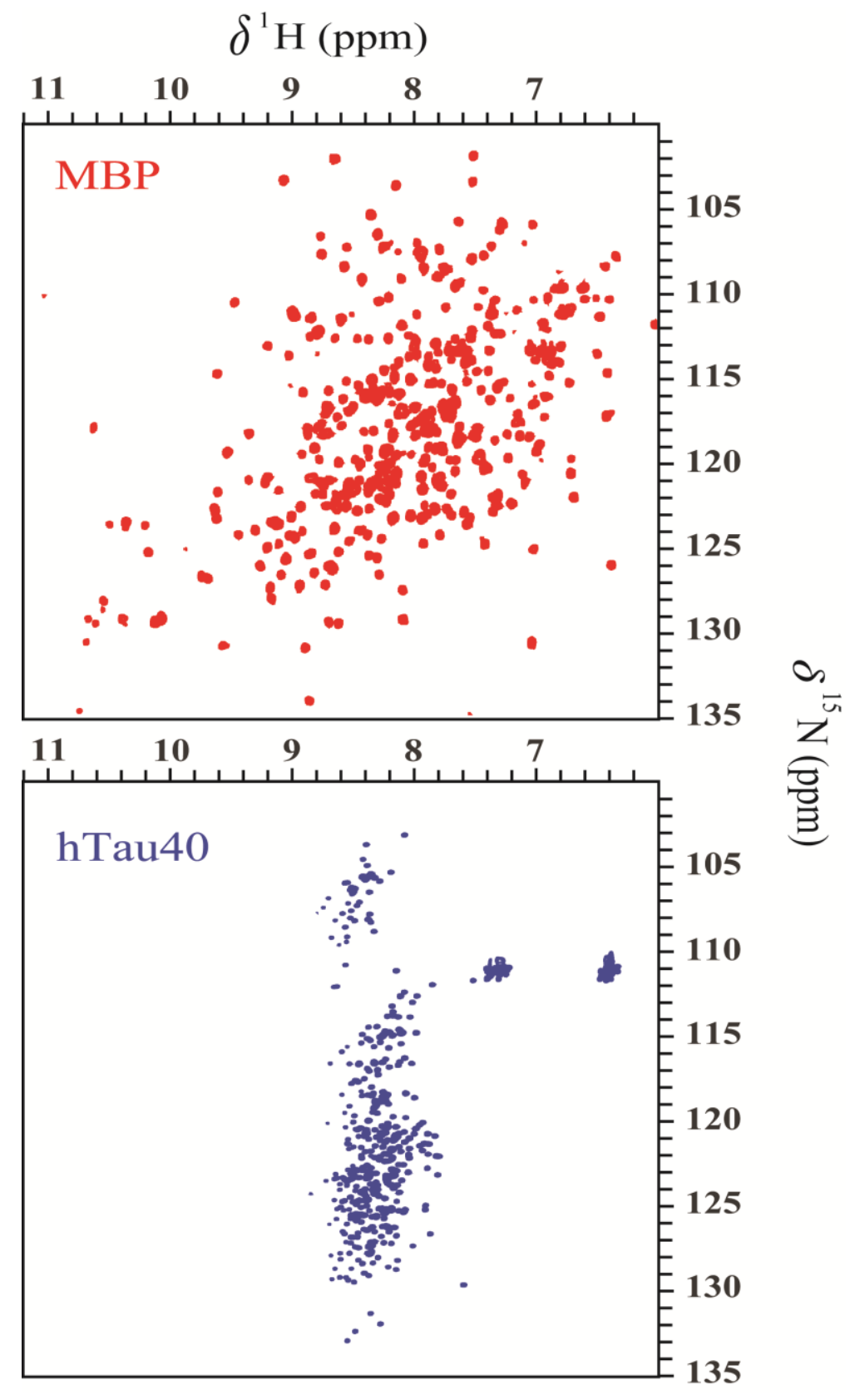

Figure 17: HSQC Spectra of Folded and Disordered Proteins.

Comparison between the $2 \mathrm{D}{ }^{1} \mathrm{H}_{-}{ }^{15} \mathrm{~N}$ HSQC spectra of 42-kDa Maltose Binding Protein $(50 \mu \mathrm{M}$ in $20 \mathrm{mM}$ phosphate buffer, $50 \mathrm{mM} \mathrm{NaCl}, 20 \mathrm{mM} \beta$-cyclodextrine; $\mathrm{pH} 7.1)$ and $45-\mathrm{kDa}$ hTau $40(50 \mu \mathrm{M}$ in $50 \mathrm{mM}$ phosphate buffer; $\mathrm{pH} 6.8$ ). Spectra were measured at $310 \mathrm{~K}$ and $278 \mathrm{~K}$, respectively on a BRUKER $700 \mathrm{MHz}$ spectrometer equipped with a cryoprobe. 


\subsubsection{Chemical Shift Mapping of Binding Sites}

Titration by NMR is a powerful technique to evaluate protein-ligand and proteinprotein interactions. The sensitivity of the backbone amide chemical shifts towards changes in their local chemical environment allows a convenient mapping of the protein's interacting sites with its partner. After recording a series of $2 \mathrm{D}{ }^{1} \mathrm{H}-{ }^{15} \mathrm{~N}$ HSQC spectra at increasing ligand concentration, the chemical shifts of the residues are monitored for possible perturbations. A partner pertubates the chemical shifts of the residues at the binding interface and may induce conformational changes (Figure 18). The mapping of the chemical shifts of both dimensions $\left(\boldsymbol{\delta} \mathrm{H}_{\mathrm{N}}\right.$ and $\left.\boldsymbol{\delta} \mathrm{N}\right)$ for an amino acid residue is commonly represented by the average calculated chemical shifts deviation $\Delta \boldsymbol{\delta} \mathrm{HN}$ (CSD):

$$
\Delta \delta_{H N}=\sqrt{\frac{\left(\Delta \delta_{N} / 5\right)^{2}+\left(\Delta \delta_{H}\right)^{2}}{2}}
$$

where $\Delta \boldsymbol{\delta}_{\boldsymbol{N}}$ is the difference between bound ligand $\left(\boldsymbol{\delta}_{\boldsymbol{N}_{\text {bound }}}\right)$ and free protein $\left(\boldsymbol{\delta}_{\boldsymbol{N}_{\text {free }}}\right)$ and $\Delta \boldsymbol{\delta}_{\boldsymbol{H}}$ is the difference between bound ligand $\left(\boldsymbol{\delta}_{\boldsymbol{H}_{\text {bound }}}\right)$ and free protein $\left(\boldsymbol{\delta}_{\boldsymbol{H}_{\text {free }}}\right)$.

Importantly, the evaluation of linewidths changes is a complementary approach to decipher chemical exchange processes such as ligand binding, protein folding and oligomerization. The rate of conformational exchange between bound and free states is estimated upon interaction of the ligand. An intensity ratio plot represents the ratios between the lineshape intensities of the bound state $\left(\boldsymbol{I}_{\text {bound }}\right)$ and $\left(\boldsymbol{I}_{\text {free }}\right)$ and the free state that are extrapolated from HSQC spectra (Figure 18). 

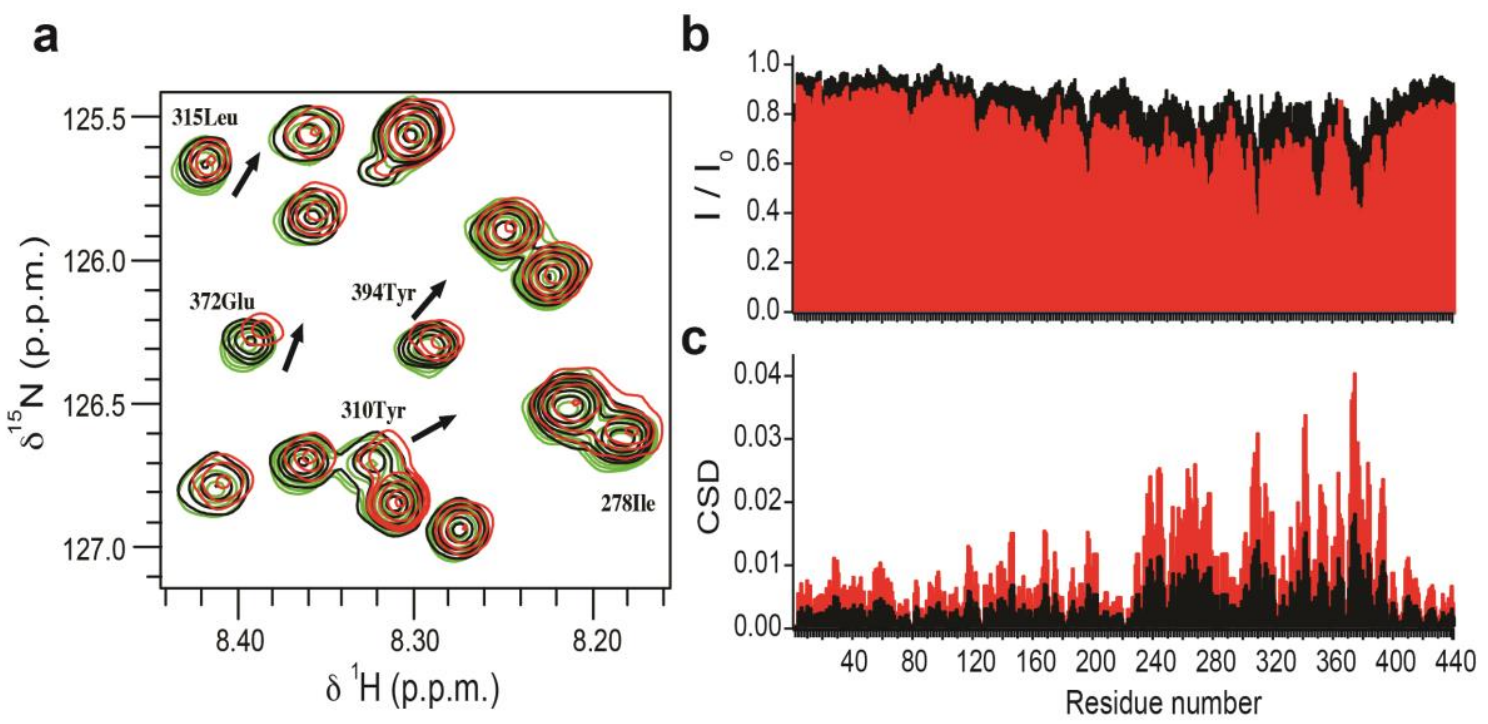

Figure 18: Mapping of Chemical Shifts and Linewidths Changes.

(a) Selected region of $2 \mathrm{D}^{1} \mathrm{H}_{-}{ }^{15} \mathrm{~N}$ HSQC spectra of hTau $40(100 \mu \mathrm{M}$ in $50 \mathrm{mM}$ phosphate buffer; $\mathrm{pH} 6.8)$ in free form (green) and at increasing concentration of PcTS (50 $\mu \mathrm{M}$, black and $100 \mu \mathrm{M}$, red). Spectra were measured at $278 \mathrm{~K}$ on a BRUKER $800 \mathrm{MHz}$ spectrometer equipped with a cryoprobe. At increasing ligand concentration, certain broadenings in NMR signals and deviations in chemical shifts (372Glu, 310Tyr, $394 \mathrm{Tyr})$ are monitored in the (b) intensity ratio and (c) chemical shift deviation plots. Modified from ${ }^{60}$. 


\subsubsection{Site-Directed Spin Labeling of Proteins}

The combination of site-directed spin labeling and NMR paramagnetic relaxation enhancement (PRE) measurements has proven to be a powerful approach for studying dynamic proteins. PRE experiments provide valuable structural constraints and quantitative interpretation of spin relaxations. Classical PRE studies often use site-directed mutagenesis to express the protein of interest with a single cysteine. Through established sulfur chemistry, a nitroxide spin label, typically $S$-(2,2,5,5-tetramethyl-2,5-dihydro- $1 \mathrm{H}$ pyrrol-3-yl) methyl methanesulfonothioate (MTSL) ${ }^{334}$ or its derivatives, is tagged to sulfhydryl of the cysteine (Figure 19). The unpaired electron of the nitroxide induces paramagnetic dipolar transverse relaxation of nearby nuclear spins that are converted into effective distances between the paramagnetic center and the electron-proton interacting spin (usually the amide protons). This long-range interaction is detected up to $25 \AA$, a clear advantage over nuclear dipolar interactions that range up to $6 \AA$. Similar to what was previously discussed for nuclear proton (Part 1.10), an unpaired electron exhibits an electronic magnetic moment $\boldsymbol{\mu}_{\boldsymbol{e}}$ related to its angular momentum $\boldsymbol{J}$ :

$$
\boldsymbol{\mu}_{\boldsymbol{e}}=\boldsymbol{g} \cdot \boldsymbol{\mu}_{B} \cdot \frac{\boldsymbol{J}}{\hbar}
$$

where $\boldsymbol{g}$ is Landé factor and $\boldsymbol{\mu}_{\boldsymbol{B}}$ is Bohr magneton (equivalent to nuclear magneton $\boldsymbol{\mu}_{\boldsymbol{N}}$ ) is defined as:

$$
\mu_{B}=\frac{e}{2 m_{e}}
$$

Where $\boldsymbol{m}_{\boldsymbol{e}}$ is the electron mass and $\boldsymbol{e}$ is its charge. The gyromagnetic ratio of the unpaired electron $\boldsymbol{\gamma}_{\boldsymbol{e}}$ is represented as:

$$
\gamma_{e}=\frac{\mu_{e}}{J}=\frac{g \cdot \mu_{B}}{\hbar}
$$




\subsection{NMR Spectroscopy}

The estimation of $\boldsymbol{\mu}_{\boldsymbol{e}}$ using the constant values of $\boldsymbol{\mu}_{\boldsymbol{B}}, \boldsymbol{g}$, and $\hbar$, reveals that the electronic magnetic moment is more than 600 times larger than the nuclear magnetic moment. ${ }^{334}$ This enhances the longitudinal and transverse relaxation rates of the nuclear spins. More importantly, the high value of $\boldsymbol{\gamma}_{\boldsymbol{e}}$ allows the measurement of long-range distance information. Both relaxation rates are indeed distance-dependent by a factor of $\boldsymbol{r}^{\mathbf{6}}$. However, internal correlation times, such as flexibility, cross relaxation and exchange processes contribute to the longitudinal relaxation rate $\boldsymbol{R}_{\mathbf{1}}$. The transverse relaxation rate $\boldsymbol{R}_{\mathbf{2}}$, on the other hand, is more reliable. ${ }^{335}$ The contribution of the unpaired electron with spin $\frac{\mathbf{1}}{\mathbf{2}}$ to the paramagnetic relaxation is dependent on the correlation time $\boldsymbol{\tau}_{\boldsymbol{c}}$ and is represented as:

$$
R_{2}^{p a r a}=\frac{1}{20}\left(\frac{\mu_{0}}{4 \pi}\right)^{2}\left(\frac{\gamma_{H}^{2} \cdot \gamma_{e}^{2} \cdot \hbar^{2}}{r^{6}}\right)\left[4 \tau_{c}+\frac{3 \tau_{c}}{1+\omega_{H}^{2} \cdot \tau_{c}^{2}}\right]
$$

Taking into account the relation between peak intensity and relaxation rate, ${ }^{336}$ PREs of the amide cross peaks can be directly measured by recording two sets of $2 \mathrm{D}{ }^{1} \mathrm{H}-{ }^{15} \mathrm{~N}$ HSQC spectra, one paramagnetic and one diamagnetic:

$$
P R E=\left(\frac{I^{\text {para }}}{I^{\text {dia }}}\right)=\left(\frac{R_{2}^{\text {dia }} \cdot e^{\left(-R_{2}^{\text {para }}\right) t}}{R_{2}^{\text {dia }}+R_{2}^{\text {para }}}\right)
$$

where $\boldsymbol{t}$ is the INEPT delay in the HSQC pulse sequence. Practically, the paramagnetic spectrum contains broadened peaks corresponding to residues in the vicinity of the attached 
MTSL spin label. After cleavage of the spin label by reducing agents, the NMR signals are recovered in the diamagnetic spectrum (Figure 19).

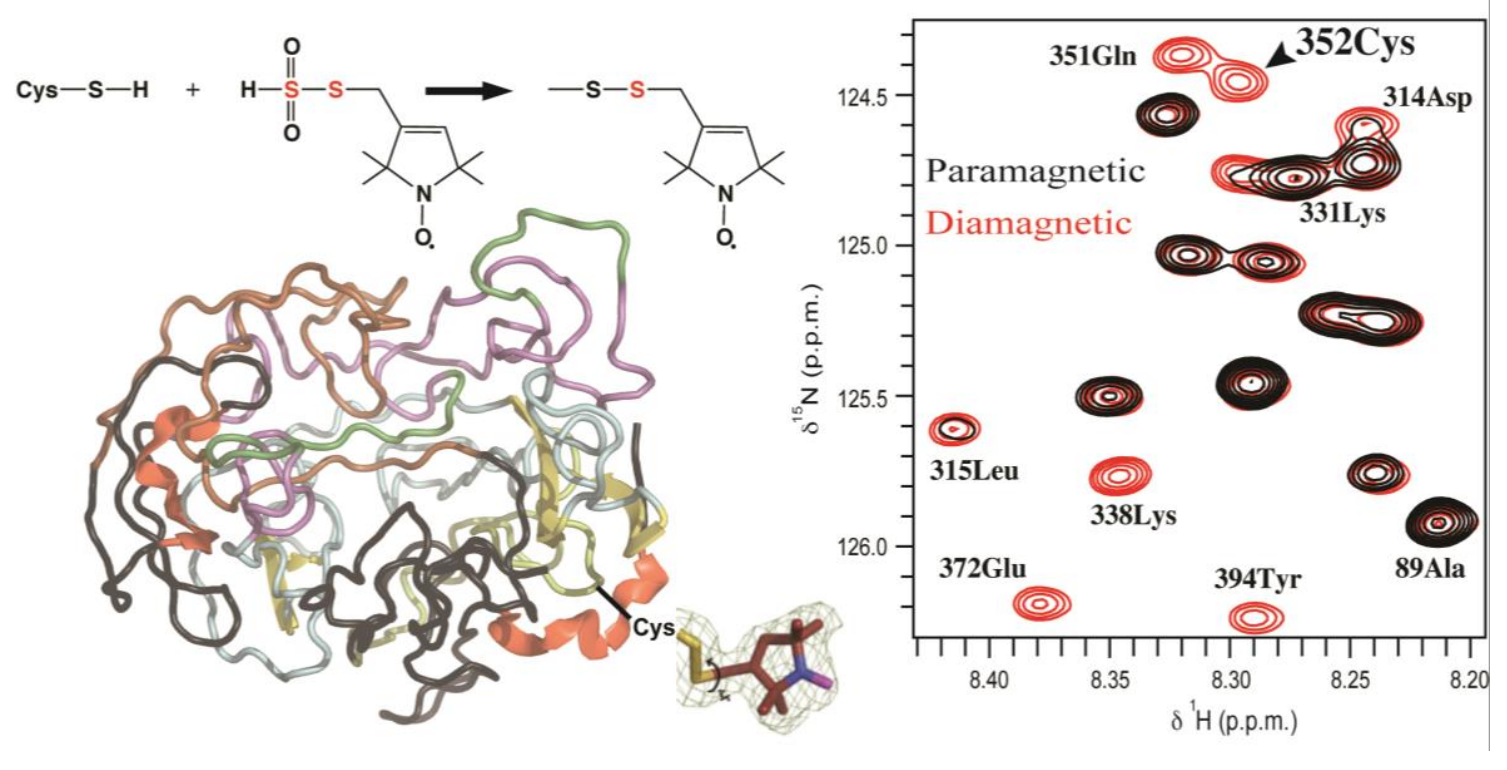

Figure 19: Site-directed Spin Labeling for PRE Measurements.

The reaction between the sulfhydryl group of the cysteine and the sulfonothioate group of the nitroxide spin label MTSL forms a disulfide bond. The unpaired electron of the nitroxide induces dipolar transverse relaxations of amide protons that report on distances up to $25 \AA$. The broadened peaks in the paramagnetic spectrum of hTau40/C291A/C322G/S352C (15 $\mu \mathrm{M}$ in $50 \mathrm{mM}$ phosphate buffer; $\mathrm{pH} 6.8)$ correspond to residues in the vicinity of the MTSL spin label attached at position C352. The addition of $1 \mathrm{mM}$ DTT cleaves the spin label and the NMR signals are recovered in the diamagnetic spectrum. Spectra were measured at 278 $\mathrm{K}$ on a BRUKER $800 \mathrm{MHz}$ spectrometer equipped with a cryoprobe. The backbone conformation of hTau40 is adapted from ${ }^{86}$. 


\subsubsection{Equilibrium Denaturation}

The process of amyloid fibrillar formation involves conformational changes of the soluble protein prior its polymerization into a common cross- $\beta$ structure. Equilibrium denaturation has been intensely employed to reveal conformational properties associated with unfolding processes, high-order assemblies, or partner binding. ${ }^{337}$ Upon titration of a denaturant such as urea or guanidinium thiocyanate $(\mathrm{GuSCN})$, the evolution of individual residues in the denaturated state can be monitored by ${ }^{1} \mathrm{H}-{ }^{15} \mathrm{~N}$ HSQC experiments. The spectral intensities of protein residues at increasing denaturant concentrations can then be fitted to different profiles based on the following criteria: (1) percentage of the initial intensities in the absence of urea from the fully denaturated state, (2) concentration of urea at which denaturation is initiated, (3) midpoint of the transition, and (4) concentration of urea at which the resonance fulfils full denaturation. One interesting application was the equilibrium denaturation of PcTS-induced Tau oligomers (Chapter 2) ${ }^{60}$ This approach revealed the distinct behavior of the residues belonging to different domains (Figure 21). For instance, T71 was fully denaturated whereas A429 was more resistant up to $1 \mathrm{M}$ urea. On the other hand, NMR T17 and K143 were partially resistant but their NMR signals progressively increased in the presence of $0.75 \mathrm{M}$ urea. It is worth noting that the broadened NMR signals of hTau40 in the oligomeric state (excess PcTS) were partially (8 M urea) or fully (3 M GuSCN) recovered (Figure 22). Nevertheless, several peaks remained resistant even at $8 \mathrm{M}$ urea. Considering the fact that the ionic nature of GuSCN establishes a higher denaturation efficiency toward systems baring electrostatic interactions. Therefore, a careful comparative investigation of urea and GuSCN denaturation activities would reveal exquisite information. 

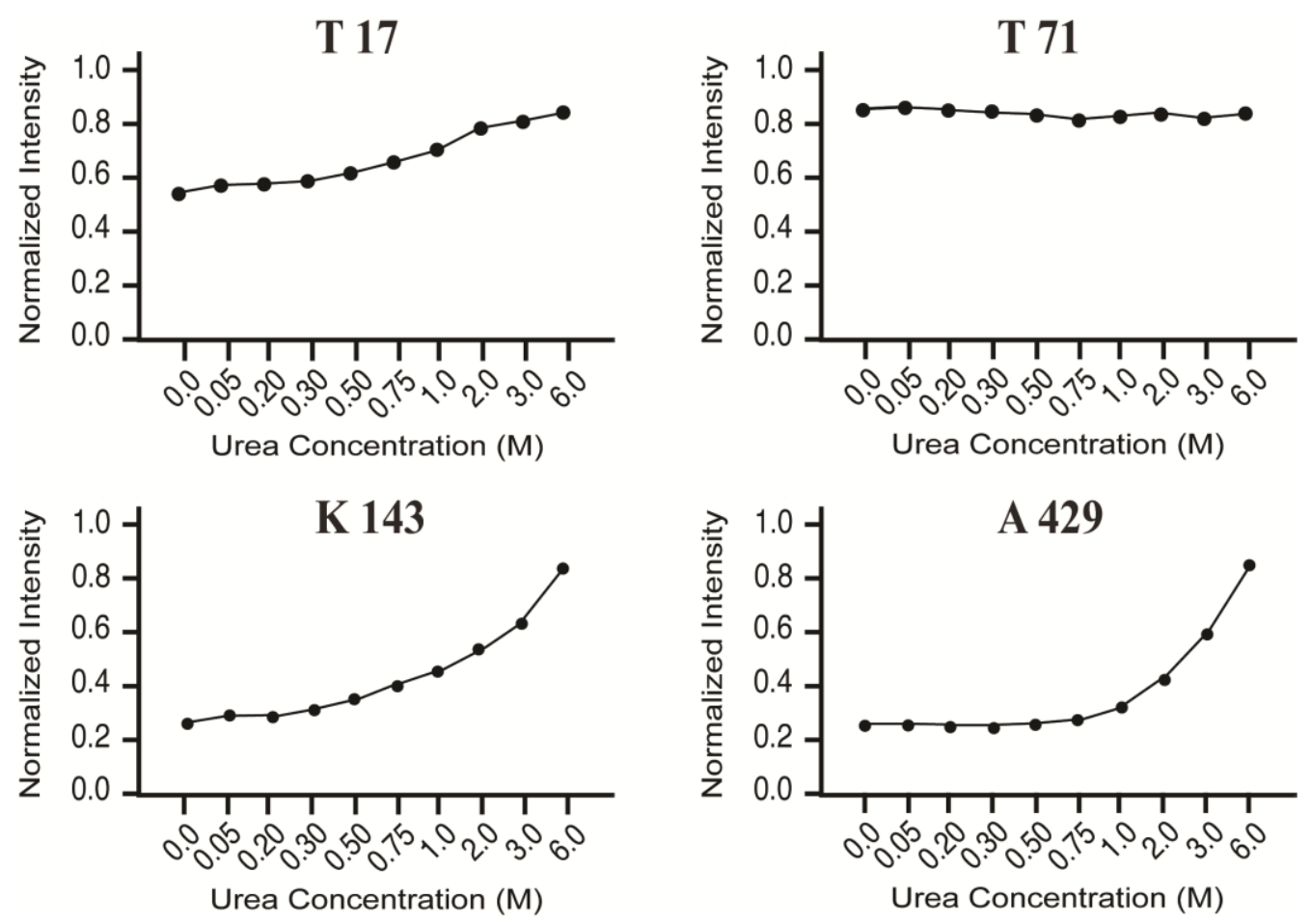

Figure 20: Urea Denaturation Profiles.

Normalized intensities from $2 \mathrm{D}{ }^{1} \mathrm{H}_{-}{ }^{15} \mathrm{~N}$ HSQC spectra of PcTS-hTau40 oligomers $(1500 \mu \mathrm{M}-100 \mu \mathrm{M}$ in 50 $\mathrm{mM}$ phosphate buffer; $\mathrm{pH}$ 6.8) are plotted at increasing urea concentrations. The data demonstrates distinct behavior of the residues between fully denaturated and partially resistant. Spectra were measured at $278 \mathrm{~K}$ on a BRUKER $800 \mathrm{MHz}$ spectrometer equipped with a cryoprobe.
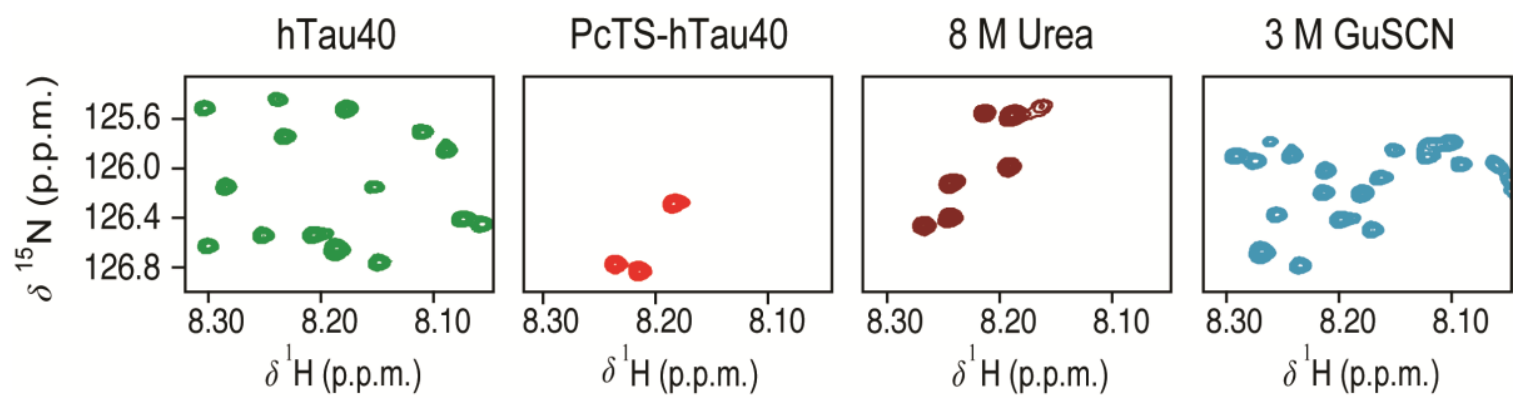

Figure 21: Equilibrium Denaturation of PcTS-stabilized Oligomers.

Selected regions of $2 \mathrm{D}{ }^{1} \mathrm{H}_{-}{ }^{15} \mathrm{~N}$ HSQC spectra of hTau40 monomer, PcTS-hTau40 oligomers without and with the addition of $8 \mathrm{M}$ urea or $3 \mathrm{M}$ GuSCN. Adapted from ${ }^{60}$. 


\subsubsection{Diffusion Ordered Spectroscopy}

The Brownian molecular motion describes the translational diffusion of a solute in solution. This phenomenon is represented by the coefficient of lateral diffusion $\boldsymbol{D}$ that depends on sample temperature, solvent viscosity, molecular shape and molecular size. In practice, molecules in solution are non-spherical solvated systems that tumble with defined dynamics. Taking this into consideration, the apparent size of the hydrated particle is then characterized by its diffusional properties using the terminology of the hydrodynamic radius $\boldsymbol{R}_{\boldsymbol{H}} \cdot{ }^{338}$ The Stokes-Einstein equation relates $\boldsymbol{R}_{\boldsymbol{H}}$ to $\boldsymbol{D}$ :

$$
D=\frac{K \cdot T}{6 \pi \cdot \eta \cdot R_{H}}
$$

where $\boldsymbol{K}$ is Boltzmann constant, $\boldsymbol{T}$ is temperature, and $\boldsymbol{\eta}$ is the viscosity of the solution. Unlike $\boldsymbol{R}_{\boldsymbol{H}}$, the well-defined parameter from crystallography, the radius of gyration $\boldsymbol{R}_{\boldsymbol{g}}$, does not include solvent effects (Figure 22) but only describes the equilibrium conformation of the total system where each atom is defined by its mass $\boldsymbol{m}_{\boldsymbol{i}}$ and coordinates $\boldsymbol{r}_{\boldsymbol{i}}$ with respect to the center of mass:

$$
\boldsymbol{R}_{g}^{2}=\frac{\sum \boldsymbol{m}_{i} x_{i}^{2}}{\sum \boldsymbol{m}_{i}}
$$

The lateral diffusion $\boldsymbol{D}$ of small organic molecules and large biomolecules are conveniently measured using Pulsed Field Gradient (PFG) NMR techniques. ${ }^{339}$ Once a magnetic gradient pulse of magnitude $\boldsymbol{g}$ is applied to the sample, the molecules are spatially encoded. After a diffusion time $\boldsymbol{\Delta}$, in which these particles freely move in solution, a second gradient pulse decodes their new coordinates. Affected by this diffusion, the intensity $\boldsymbol{I}$ of detected NMR signal is then attenuated at a degree proportional to $\boldsymbol{\Delta}$ and $\boldsymbol{g}:$

$$
I=I_{0} \exp \left[-D \cdot \gamma^{2} \cdot g^{2} \cdot \delta^{2}(\Delta-\delta / 3)\right]
$$


where $\boldsymbol{\delta}$ is the duration of the magnetic pulse. (Figure 22) The most dominant pulse sequences used in PFG-NMR diffusion measurements are derived from the stimulated echo (STE) pulse sequence that reduces artifacts in the diffusion spectra by employing bipolar pairs of gradient pulses and can readily incorporate a WATERGATE solvent suppression. ${ }^{340}$
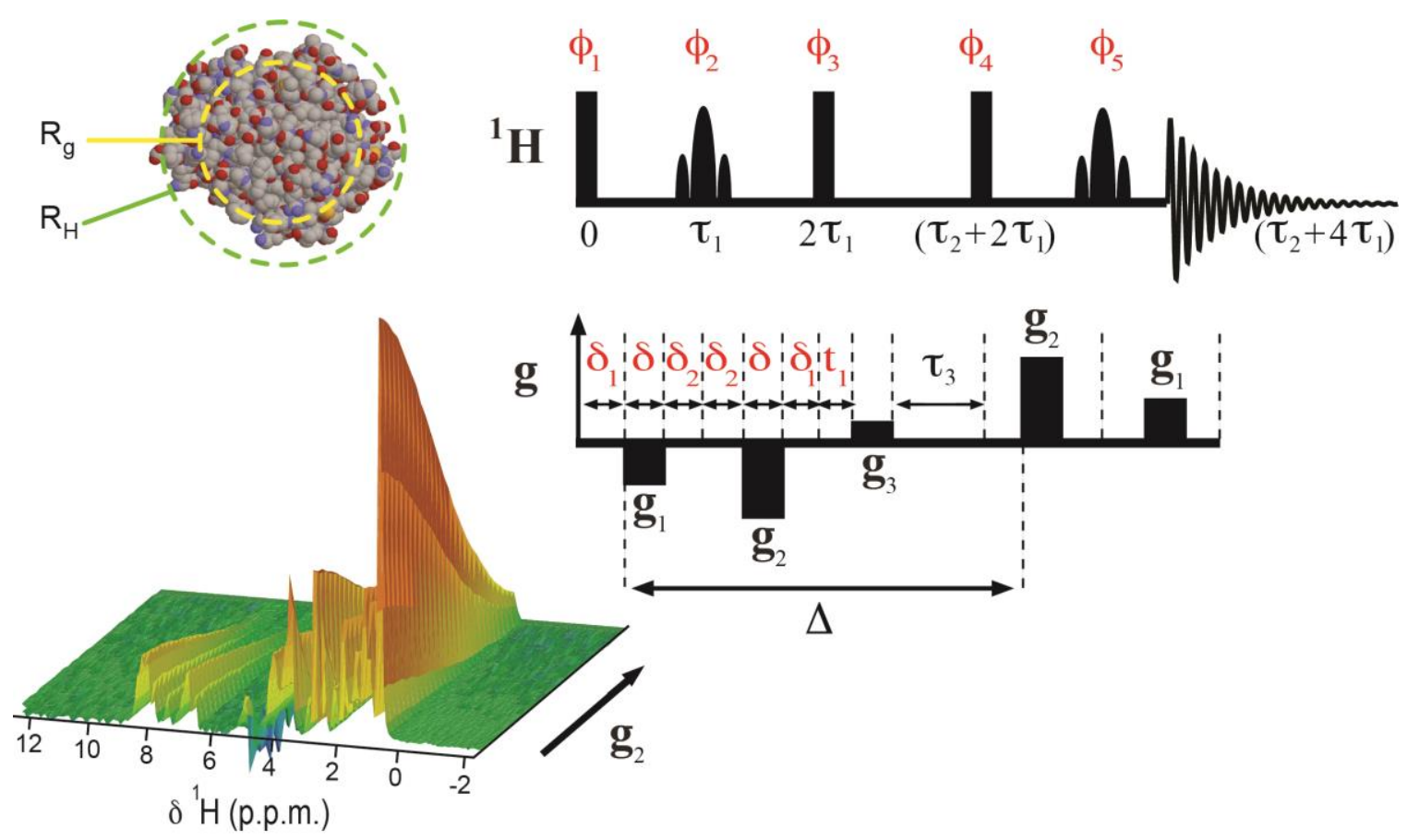

\section{Figure 22: Diffusion Ordered Spectroscopy.}

Comparison of the hydrodynamic radius $\mathrm{R}_{\mathrm{H}}$ and radius of gyration $\mathrm{R}_{\mathrm{g}}$ in a protein. Pseudo $2 \mathrm{D}{ }^{1} \mathrm{H}$ DOSY spectra of hTau40 measured by the PGSTE-WATERGATE sequence where $g_{1}, g_{2}$ and $g_{3}$ are rectangular gradient pulses with different amplitudes, and the shaped rectangles represent "W5" binomial $\pi$ pulses. The phase cycle for the pulse sequences is $\phi_{1}=x,-x ; \phi_{2}=y, y,-x,-x ; \phi_{3}=x, y,-x,-y ; \phi_{4}=x, y,-x$, $-y ; \phi_{5}=y ; \phi_{r}$ (receiver phase) $=x,-x,-x, x$. For $\phi_{2}=y\left(\right.$ or $\left.\phi_{5}=y\right)$, the phasing of the "W5" binomial pulse train is $(x)_{5}-(-x)_{5}$; for $\phi_{2}=-x$, the phasing is $(y)_{5}-(-y)_{5}$. Spectra of hTau40 $(40 \mu \mathrm{M}$ in $50 \mathrm{mM}$ phosphate buffer; $\mathrm{pH} 6.8,10 \% \mathrm{D}_{2} \mathrm{O}$ ) were measured at $278 \mathrm{~K}$ on a BRUKER $600 \mathrm{MHz}$ spectrometer equipped with a cryoprobe using the optimized acquisition parameters: 128 scans, $16 \mathrm{~K}$ complex points, spectral width of $8992 \mathrm{~Hz}$, gradient strength from $2 \%$ to $95 \%$ of the maximum gradient strength $(55.14 \mathrm{G} / \mathrm{cm})$ in 30 steps with $\Delta=180 \mathrm{~ms}$ and $\delta=6.5 \mathrm{~ms}$. Pulse sequence adapted from ${ }^{340}$. 


\subsection{Complementary Biophysical Techniques}

\subsubsection{Dynamic Light Scattering}

The fundamental concept of photon correlation spectroscopy, widely known as Dynamic light scattering (DLS) is explained by the semi-classical light scattering theory. ${ }^{341}$ The electric field of a monochromatic light impinging on particles in solution prompts the electrons of these molecules to adapt an oscillating polarization. Depending on size distribution and particle shape, the polarized molecules scatter the incoming light and determine its frequency shifts (Doppler Shifts), polarization and intensity. DLS measures the Brownian translational diffusion. The measured diffusion coefficients are then fitted to an autocorrelation function adapted from statistical mechanics to reconstruct size distribution and describe the particle's motion.

\subsubsection{Fourier Transform Infrared and Circular Dichroism}

Fourier Transform Infrared (FTIR) and Circular Dichroism (CD) are two vibrational spectroscopies that allow analysis of protein secondary structure. FTIR is a powerful technique that has been used to demonstrate characteristic differences in the molecular arrangement of $\beta$-sheets in amyloid fibrils and native proteins. ${ }^{342}$ In the spectral region, amide I and II vibrational bands are most sensitive to the secondary structural components of the protein backbone (Figure 23). ${ }^{343}$ The amide I band corresponds to $\mathrm{C}=\mathrm{O}$ stretching vibrations of the peptide linkages, appears over the range 1700 to $1600 \mathrm{~cm}^{-1}$ and its characteristic frequency component correlates highly to each of the structural elements. In contrast, the amide II band derives mainly from in-plane $\mathrm{NH}$ bending and $\mathrm{CN}$ stretching 


\subsection{Complementary Biophysical Techniques}

vibrations and displays much less sensitivity towards protein conformation. Depending on the nature of side chains and hydrogen bonding involved, the additional amide vibrational bands become very complex to interpret.

CD spectroscopy is the most utilized technique for determination of the secondary structural composition and folding properties of peptides and proteins. Whether purified from biological tissues or recombinantly expressed, CD determines protein stability and conformational modifications. Each of the structural elements has a characteristic CD spectral signature reflecting the molar ellipticity (Figure 23). ${ }^{344}$ The spectrum of a typical $\alpha$-helical protein displays two negative bands (222 and $208 \mathrm{~nm}$ ) and one positive (193 $\mathrm{nm})$. A protein with $\beta$ - pleated sheets shows two bands, one negative $(218 \mathrm{~nm})$ and one positive $(195 \mathrm{~nm})$. On the other hand, a disordered protein exhibits a low ellipticity at $210 \mathrm{~nm}$ and

a negative band at $195 \mathrm{~nm}$. A triple helix protein has a typical extended helical conformation. The low overall ellipticity reflects a protein's denaturated state. 


\subsection{Complementary Biophysical Techniques}

a

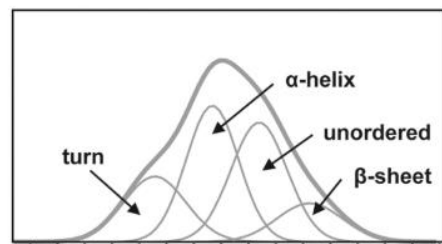

17201700168016601640162016001580 Wavenumber $\left(\mathrm{cm}^{-1}\right)$

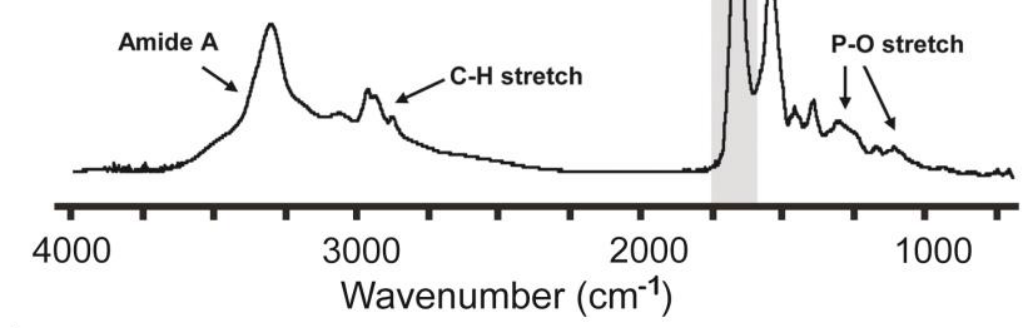

b

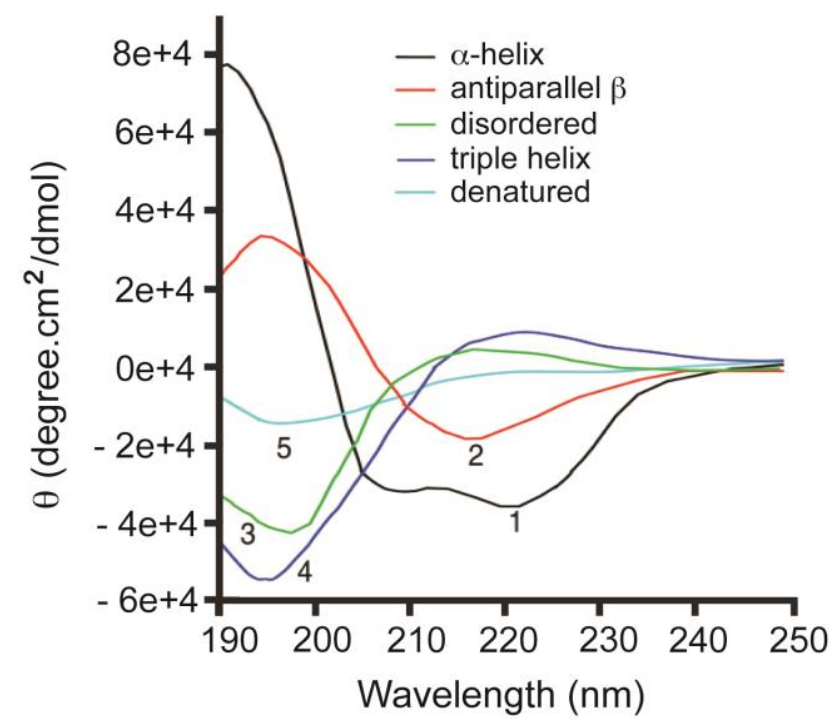

Figure 23: FTIR and CD Vibrational Spectroscopies.

(a) The two vibrational bands in FTIR spectra (amide I from $\mathrm{C}=\mathrm{O}$ stretching vibrations and amide II from in-plane $\mathrm{NH}$ bending and $\mathrm{CN}$ stretching vibrations) are most sensitive to the secondary structural components of the protein backbone. (b) CD spectroscopy determines the secondary structural composition with spectral signatures reflecting the molar ellipticities of $\alpha$-helical, $\beta$ - pleated sheets, disordered and extended helical conformations. FTIR and CD spectra are respectively adapted from ${ }^{343}$ and ${ }^{344}$. 


\subsubsection{Small Angle X-ray Scattering}

Small angle X-ray scattering (SAXS) has emerged in the field of structural biology as a complementary method to characterize the overall size and shape of biomolecules in solution. The basic principle of SAXS involves illuminating the solution with a monochromatic x-ray source and measurement of the intensity at different scattering angles. The analysis of the angular-dependent scattered intensities will then reveal the structural information of the particle. Practically, from the so-called Guinier's plot the average radius of gyration of the biomolecule is calculated. The Kratky plot reports whether the molecules are in a disordered state or a globular protein. ${ }^{345}$ Moreover, Fourier transformation of the scattering profile provides information about the distance distribution function $p(r)$ associated to the maximum distance $D_{\max }$ within the particle and offers a rough estimation of the particle shape. Recent improvements enabled the technique to study not only monodispersed samples but also oligomeric mixtures. ${ }^{346,347}$

In case of folded proteins, the three dimensional structure can be refined by incorporating the scattering profile in a computational study. The overall shape is then calculated using either the $a b$ initio method ${ }^{348}$ or the rigid body modeling. ${ }^{349}$ On the other hand, SAXS studies of IDP proteins combine other techniques such as the Ensemble optimization method (EOM) $)^{350}$ to generate a pool of random conformations within a defined landscape. This involves sampling a conformational space to select sub-ensembles that best fit to the experimental results. IDPs are characterized by large $\mathrm{R}_{\mathrm{g}}$ and $\mathrm{D}_{\max }$ values and an expanded $p(r)$. 


\subsection{Complementary Biophysical Techniques}
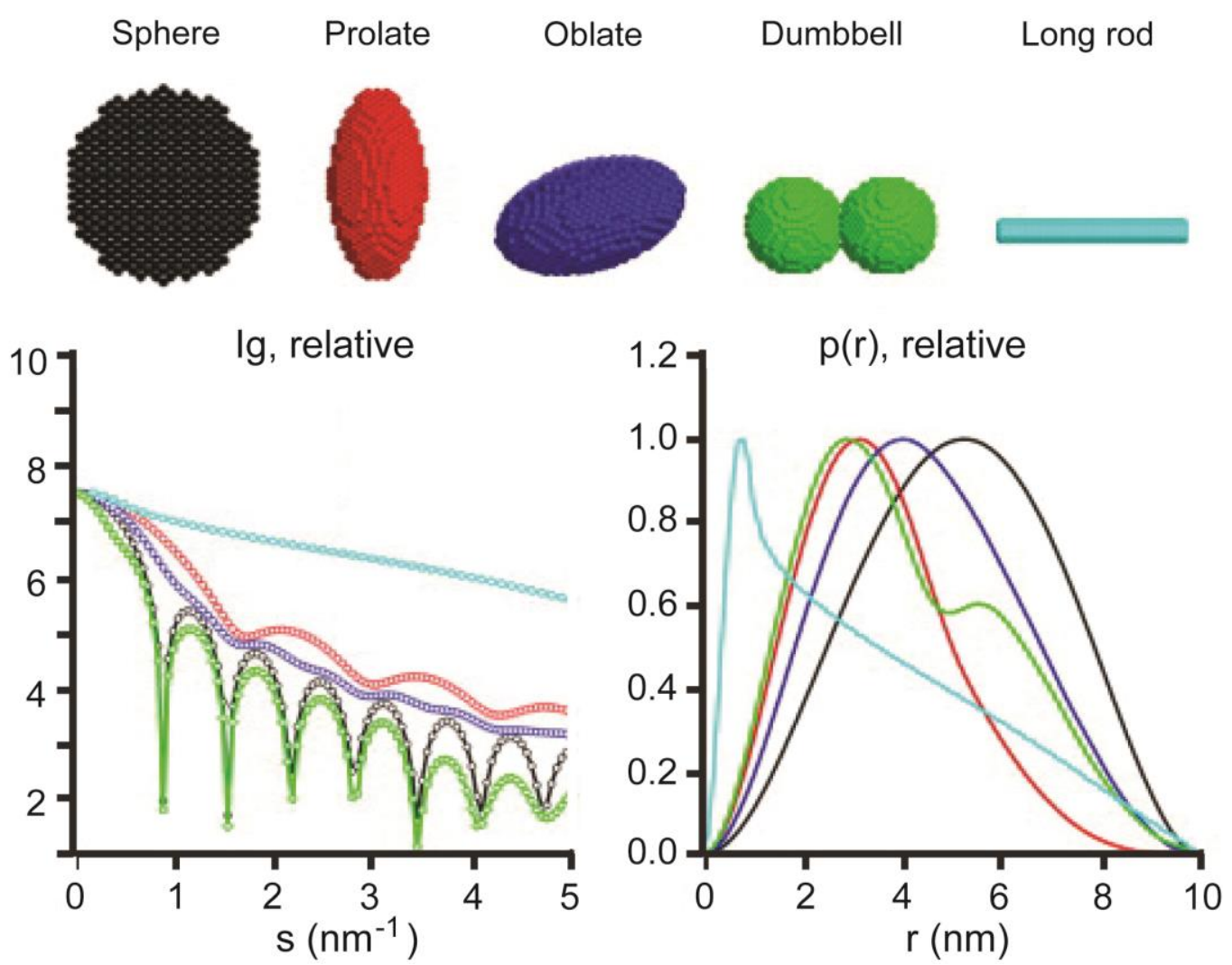

Figure 24: Small Angle X-ray Scattering and Geometric Structures.

Scattering intensities and distance distribution functions $p(r)$ calculated for typical geometric shapes are shown together with the bead models. Adapted from ${ }^{345}$. 


\section{References}

1 C. B. Anfinsen. Principles that govern the folding of protein chains. Science 181, 223-230 (1973).

2 F. U. Hartl. Molecular chaperones in cellular protein folding. Nature 381, 571-579, doi:10.1038/381571a0 (1996).

3 K. A. Dill \& J. L. MacCallum. The protein-folding problem, 50 years on. Science 338, 1042-1046, doi:10.1126/science.1219021 (2012).

4 M. Stefani. Protein misfolding and aggregation: new examples in medicine and biology of the dark side of the protein world. Biochim Biophys Acta 1739, 5-25, doi:10.1016/j.bbadis.2004.08.004 (2004).

5 C. M. Dobson. Principles of protein folding, misfolding and aggregation. Semin Cell Dev Biol 15, 3-16, doi:10.1016/j.semcdb.2003.12.008 (2004).

6 T. R. Jahn \& S. E. Radford. The Yin and Yang of protein folding. FEBS J 272, 5962-5970, doi:10.1111/j.1742-4658.2005.05021.x (2005).

7 F. U. Hartl \& M. Hayer-Hartl. Converging concepts of protein folding in vitro and in vivo. Nat Struct Mol Biol 16, 574-581, doi:10.1038/nsmb.1591 (2009).

8 C. A. Ross \& M. A. Poirier. Opinion: What is the role of protein aggregation in neurodegeneration? Nat Rev Mol Cell Biol 6, 891-898, doi:10.1038/nrm1742 (2005).

9 V. N. Uversky \& A. L. Fink. Conformational constraints for amyloid fibrillation: the importance of being unfolded. Biochim Biophys Acta 1698, 131-153, doi:10.1016/j.bbapap.2003.12.008 (2004).

10 J. C. Young, V. R. Agashe, K. Siegers \& F. U. Hartl. Pathways of chaperonemediated protein folding in the cytosol. Nat Rev Mol Cell Biol 5, 781-791, doi:10.1038/nrm1492 (2004).

11 T. Maier, L. Ferbitz, E. Deuerling \& N. Ban. A cradle for new proteins: trigger factor at the ribosome. Curr Opin Struct Biol 15, 204-212, doi:10.1016/j.sbi.2005.03.005 (2005).

12 C. M. Pickart \& R. E. Cohen. Proteasomes and their kin: proteases in the machine age. Nat Rev Mol Cell Biol 5, 177-187, doi:10.1038/nrm1336 (2004).

13 A. Alzheimer. Über eine eigenartige Erkrankung de Hirnrinde. Allgemeine Zeitschrift für Psychiatrie und Psychisch-gerichtliche Medizin 64, 146-148 (1907).

14 O. Fischer. Miliare Nekrosen mit drusigen Wucherungen der Neurofibrillen, eine regelmässige Veränderung der Hirnrinde bei seniler Demenz. Monatsschr Psychiat Neurol 22, 361-372 (1907).

15 M. Goedert \& B. Ghetti. Alois Alzheimer: his life and times. Brain Pathol 17, 57 62, doi:10.1111/j.1750-3639.2007.00056.x (2007).

16 A. A. Über eigenartige Krankheitsfälle des späteren Alters. Z ges Neurol Psychiat. 4, 356-385 (1911). 
17 F. Lewy. Paralysis agitans. In: Max Lewandowsky (Hrsg.). Handbuch der Neurologie, Band I Pathologische Anatomie, Berlin, Springer, 920-933 (1912).

18 M. Goedert, M. G. Spillantini, K. Del Tredici \& H. Braak. 100 years of Lewy pathology. Nat Rev Neurol 9, 13-24, doi:10.1038/nrneurol.2012.242 (2013).

19 C. Soto. Unfolding the role of protein misfolding in neurodegenerative diseases. Nat Rev Neurosci 4, 49-60, doi:10.1038/nrn1007 (2003).

20 G. G. Glenner \& C. W. Wong. Alzheimer's disease: initial report of the purification and characterization of a novel cerebrovascular amyloid protein. Biochem Biophys Res Commun 120, 885-890 (1984).

21 I. Grundke-Iqbal et al. Microtubule-associated protein tau. A component of Alzheimer paired helical filaments. J Biol Chem 261, 6084-6089 (1986).

22 M. G. Spillantini et al. Alpha-synuclein in Lewy bodies. Nature 388, 839-840, doi:10.1038/42166 (1997).

23 M. DiFiglia et al. Aggregation of huntingtin in neuronal intranuclear inclusions and dystrophic neurites in brain. Science 277, 1990-1993 (1997).

24 D. C. Bolton, M. P. McKinley \& S. B. Prusiner. Identification of a protein that purifies with the scrapie prion. Science 218, 1309-1311 (1982).

25 L. I. Bruijn et al. Aggregation and motor neuron toxicity of an ALS-linked SOD1 mutant independent from wild-type SOD1. Science 281, 1851-1854 (1998).

26 C. Haass \& D. J. Selkoe. Soluble protein oligomers in neurodegeneration: lessons from the Alzheimer's amyloid beta-peptide. Nat Rev Mol Cell Biol 8, 101-112, doi:10.1038/nrm2101 (2007).

27 C. A. Ross \& M. A. Poirier. Protein aggregation and neurodegenerative disease. Nat Med 10 Suppl, S10-17, doi:10.1038/nm1066 (2004).

28 D. M. Skovronsky, V. M. Lee \& J. Q. Trojanowski. Neurodegenerative diseases: new concepts of pathogenesis and their therapeutic implications. Annu Rev Pathol 1, 151-170, doi:10.1146/annurev.pathol.1.110304.100113 (2006).

29 B. S. Gadad, G. B. Britton \& K. S. Rao. Targeting oligomers in neurodegenerative disorders: lessons from alpha-synuclein, tau, and amyloid-beta peptide. $J$ Alzheimers Dis 24 Suppl 2, 223-232, doi:10.3233/JAD-2011-110182 (2011).

30 B. Caughey \& P. T. Lansbury. Protofibrils, pores, fibrils, and neurodegeneration: separating the responsible protein aggregates from the innocent bystanders. Annu Rev Neurosci 26, 267-298, doi:10.1146/annurev.neuro.26.010302.081142 (2003).

31 G. A. Papoian. Proteins with weakly funneled energy landscapes challenge the classical structure-function paradigm. Proc Natl Acad Sci U S A 105, 14237-14238, doi:10.1073/pnas.0807977105 (2008).

32 C. J. Oldfield et al. Comparing and combining predictors of mostly disordered proteins. Biochemistry 44, 1989-2000, doi:10.1021/bi047993o (2005).

33 P. Tompa. The interplay between structure and function in intrinsically unstructured proteins. FEBS Lett 579, 3346-3354, doi:10.1016/j.febslet.2005.03.072 (2005). 
34 P. E. Wright \& H. J. Dyson. Intrinsically unstructured proteins: re-assessing the protein structure-function paradigm. $J$ Mol Biol 293, 321-331, doi:10.1006/jmbi.1999.3110 (1999).

35 P. Tompa. Intrinsically unstructured proteins. Trends Biochem Sci 27, 527-533 (2002).

36 V. N. Uversky, C. J. Oldfield \& A. K. Dunker. Intrinsically disordered proteins in human diseases: introducing the D2 concept. Annu Rev Biophys 37, 215-246, doi:10.1146/annurev.biophys.37.032807.125924 (2008).

37 A. L. Fink. Natively unfolded proteins. Curr Opin Struct Biol 15, 35-41, doi:10.1016/j.sbi.2005.01.002 (2005).

38 P. Radivojac et al. Intrinsic disorder and functional proteomics. Biophys J 92, 14391456, doi:10.1529/biophysj.106.094045 (2007).

39 P. Romero et al. Sequence complexity of disordered protein. Proteins 42, 38-48 (2001).

40 Z. Obradovic et al. Predicting intrinsic disorder from amino acid sequence. Proteins 53 Suppl 6, 566-572, doi:10.1002/prot.10532 (2003).

41 H. J. Dyson \& P. E. Wright. Insights into the structure and dynamics of unfolded proteins from nuclear magnetic resonance. Adv Protein Chem 62, 311-340 (2002).

42 A. K. Dunker, Z. Obradovic, P. Romero, E. C. Garner \& C. J. Brown. Intrinsic protein disorder in complete genomes. Genome Inform Ser Workshop Genome Inform 11, 161-171 (2000).

43 A. K. Dunker et al. The unfoldomics decade: an update on intrinsically disordered proteins. BMC Genomics 9 Suppl 2, S1, doi:10.1186/1471-2164-9-S2-S1 (2008).

44 H. J. Dyson \& P. E. Wright. Intrinsically unstructured proteins and their functions. Nat Rev Mol Cell Biol 6, 197-208, doi:10.1038/nrm1589 (2005).

45 P. Tompa. Structural disorder in amyloid fibrils: its implication in dynamic interactions of proteins. FEBS $J$ 276, 5406-5415, doi:10.1111/j.17424658.2009.07250.x (2009).

46 H. J. Dyson \& P. E. Wright. Coupling of folding and binding for unstructured proteins. Curr Opin Struct Biol 12, 54-60 (2002).

47 P. E. Wright \& H. J. Dyson. Linking folding and binding. Curr Opin Struct Biol 19, 31-38, doi:10.1016/j.sbi.2008.12.003 (2009).

48 M. D. Weingarten, A. H. Lockwood, S. Y. Hwo \& M. W. Kirschner. A protein factor essential for microtubule assembly. Proc Natl Acad Sci U S A 72, 1858-1862 (1975).

49 G. Lee, N. Cowan \& M. Kirschner. The primary structure and heterogeneity of tau protein from mouse brain. Science 239, 285-288 (1988).

50 R. L. Neve, P. Harris, K. S. Kosik, D. M. Kurnit \& T. A. Donlon. Identification of cDNA clones for the human microtubule-associated protein tau and chromosomal localization of the genes for tau and microtubule-associated protein 2. Brain Res 387, 271-280 (1986). 
51 M. Goedert, M. G. Spillantini, R. Jakes, D. Rutherford \& R. A. Crowther. Multiple isoforms of human microtubule-associated protein tau: sequences and localization in neurofibrillary tangles of Alzheimer's disease. Neuron 3, 519-526 (1989).

52 M. G. Spillantini \& M. Goedert. Tau pathology and neurodegeneration. The Lancet Neurology 12, 609-622, doi:10.1016/s1474-4422(13)70090-5 (2013).

53 M. Goedert \& R. Jakes. Expression of separate isoforms of human tau protein: correlation with the tau pattern in brain and effects on tubulin polymerization. EMBO J 9, 4225-4230 (1990).

54 B. L. Goode et al. Functional interactions between the proline-rich and repeat regions of tau enhance microtubule binding and assembly. Mol Biol Cell 8, 353365 (1997).

55 B. Trinczek, J. Biernat, K. Baumann, E. M. Mandelkow \& E. Mandelkow. Domains of tau protein, differential phosphorylation, and dynamic instability of microtubules. Mol Biol Cell 6, 1887-1902 (1995).

56 M. D. Mukrasch et al. The "jaws" of the tau-microtubule interaction. J Biol Chem 282, 12230-12239, doi:10.1074/jbc.M607159200 (2007).

57 N. Hirokawa, Y. Shiomura \& S. Okabe. Tau proteins: the molecular structure and mode of binding on microtubules. J Cell Biol 107, 1449-1459 (1988).

58 S. Barghorn \& E. Mandelkow. Toward a unified scheme for the aggregation of tau into Alzheimer paired helical filaments. Biochemistry 41, 14885-14896 (2002).

59 M. Arrasate, M. Perez, J. M. Valpuesta \& J. Avila. Role of glycosaminoglycans in determining the helicity of paired helical filaments. Am J Pathol 151, 1115-1122 (1997).

60 E. Akoury et al. Inhibition of tau filament formation by conformational modulation. J Am Chem Soc 135, 2853-2862, doi:10.1021/ja312471h (2013).

61 K. S. Kosik, L. D. Orecchio, S. Bakalis \& R. L. Neve. Developmentally regulated expression of specific tau sequences. Neuron 2, 1389-1397 (1989).

62 J. Avila, F. Lim, F. Moreno, C. Belmonte \& A. C. Cuello. Tau function and dysfunction in neurons: its role in neurodegenerative disorders. Mol Neurobiol 25, 213-231, doi:10.1385/MN:25:3:213 (2002).

63 R. Brandt, J. Leger \& G. Lee. Interaction of tau with the neural plasma membrane mediated by tau's amino-terminal projection domain. J Cell Biol 131, 1327-1340 (1995).

64 J. A. Greenwood \& G. V. Johnson. Localization and in situ phosphorylation state of nuclear tau. Exp Cell Res 220, 332-337, doi:10.1006/excr.1995.1323 (1995).

65 D. Trabzuni et al. MAPT expression and splicing is differentially regulated by brain region: relation to genotype and implication for tauopathies. Hum Mol Genet 21, 4094-4103, doi:10.1093/hmg/dds238 (2012).

66 Y. Gu, F. Oyama \& Y. Ihara. Tau is widely expressed in rat tissues. J Neurochem 67, 1235-1244 (1996).

67 S. Sangrajrang et al. Estramustine resistance correlates with tau over-expression in human prostatic carcinoma cells. Int J Cancer 77, 626-631 (1998). 
68 S. Souter \& G. Lee. Microtubule-associated protein tau in human prostate cancer cells: isoforms, phosphorylation, and interactions. J Cell Biochem 108, 555-564, doi:10.1002/jcb.22287 (2009).

69 R. Rouzier et al. Microtubule-associated protein tau: a marker of paclitaxel sensitivity in breast cancer. Proc Natl Acad Sci U S A 102, 8315-8320, doi:10.1073/pnas.0408974102 (2005).

70 K. Mimori et al. Reduced tau expression in gastric cancer can identify candidates for successful Paclitaxel treatment. $B r \quad J$ Cancer 94, 1894-1897, doi:10.1038/sj.bjc.6603182 (2006).

71 A. Jimeno et al. Development of two novel benzoylphenylurea sulfur analogues and evidence that the microtubule-associated protein tau is predictive of their activity in pancreatic cancer. Mol Cancer Ther 6, 1509-1516, doi:10.1158/15357163.MCT-06-0592 (2007).

72 M. Hasegawa et al. Protein sequence and mass spectrometric analyses of tau in the Alzheimer's disease brain. J Biol Chem 267, 17047-17054 (1992).

73 W. H. Stoothoff \& G. V. Johnson. Tau phosphorylation: physiological and pathological consequences. Biochim Biophys Acta 1739, 280-297, doi:10.1016/j.bbadis.2004.06.017 (2005).

74 L. Martin, X. Latypova \& F. Terro. Post-translational modifications of tau protein: implications for Alzheimer's disease. Neurochem Int 58, 458-471, doi:10.1016/j.neuint.2010.12.023 (2011).

75 P. Seubert et al. Detection of phosphorylated Ser262 in fetal tau, adult tau, and paired helical filament tau. J Biol Chem 270, 18917-18922 (1995).

76 D. P. Hanger, B. H. Anderton \& W. Noble. Tau phosphorylation: the therapeutic challenge for neurodegenerative disease. Trends Mol Med 15, 112-119, doi:10.1016/j.molmed.2009.01.003 (2009).

77 D. P. Hanger, K. Hughes, J. R. Woodgett, J. P. Brion \& B. H. Anderton. Glycogen synthase kinase-3 induces Alzheimer's disease-like phosphorylation of tau: generation of paired helical filament epitopes and neuronal localisation of the kinase. Neurosci Lett 147, 58-62 (1992).

78 J. J. Lucas et al. Decreased nuclear beta-catenin, tau hyperphosphorylation and neurodegeneration in GSK-3beta conditional transgenic mice. EMBO J 20, 27-39, doi:10.1093/emboj/20.1.27 (2001).

79 G. Drewes et al. Mitogen activated protein (MAP) kinase transforms tau protein into an Alzheimer-like state. EMBO J 11, 2131-2138 (1992).

80 I. Correas, J. Diaz-Nido \& J. Avila. Microtubule-associated protein tau is phosphorylated by protein kinase $\mathrm{C}$ on its tubulin binding domain. J Biol Chem 267, 15721-15728 (1992).

81 G. Drewes, A. Ebneth, U. Preuss, E. M. Mandelkow \& E. Mandelkow. MARK, a novel family of protein kinases that phosphorylate microtubule-associated proteins and trigger microtubule disruption. Cell 89, 297-308 (1997). 
82 S. Illenberger et al. The endogenous and cell cycle-dependent phosphorylation of tau protein in living cells: implications for Alzheimer's disease. Mol Biol Cell 9, 1495-1512 (1998).

83 T. Li \& H. K. Paudel. Glycogen synthase kinase 3beta phosphorylates Alzheimer's disease-specific Ser396 of microtubule-associated protein tau by a sequential mechanism. Biochemistry 45, 3125-3133, doi:10.1021/bi051634r (2006).

84 O. Schweers, E. Schonbrunn-Hanebeck, A. Marx \& E. Mandelkow. Structural studies of tau protein and Alzheimer paired helical filaments show no evidence for beta-structure. J Biol Chem 269, 24290-24297 (1994).

85 D. W. Cleveland, S. Y. Hwo \& M. W. Kirschner. Physical and chemical properties of purified tau factor and the role of tau in microtubule assembly. J Mol Biol 116, 227-247 (1977).

86 M. D. Mukrasch et al. Structural polymorphism of 441-residue tau at single residue resolution. PLoS Biol 7, e34, doi:10.1371/journal.pbio.1000034 (2009).

87 M. D. Mukrasch et al. Sites of tau important for aggregation populate \{beta\}structure and bind to microtubules and polyanions. J Biol Chem 280, 24978-24986, doi:10.1074/jbc.M501565200 (2005).

88 M. D. Mukrasch et al. Highly populated turn conformations in natively unfolded tau protein identified from residual dipolar couplings and molecular simulation. $J$ Am Chem Soc 129, 5235-5243, doi:10.1021/ja0690159 (2007).

89 P. Barre \& D. Eliezer. Folding of the repeat domain of tau upon binding to lipid surfaces. J Mol Biol 362, 312-326, doi:10.1016/j.jmb.2006.07.018 (2006).

90 O. C. Andronesi et al. Characterization of Alzheimer's-like paired helical filaments from the core domain of tau protein using solid-state NMR spectroscopy. $J$ Am Chem Soc 130, 5922-5928, doi:10.1021/ja7100517 (2008).

91 V. Daebel et al. beta-Sheet core of tau paired helical filaments revealed by solidstate NMR. J Am Chem Soc 134, 13982-13989, doi:10.1021/ja305470p (2012).

92 B. Xue, R. L. Dunbrack, R. W. Williams, A. K. Dunker \& V. N. Uversky. PONDRFIT: a meta-predictor of intrinsically disordered amino acids. Biochim Biophys Acta 1804, 996-1010, doi:10.1016/j.bbapap.2010.01.011 (2010).

93 T. Ishida \& K. Kinoshita. Prediction of disordered regions in proteins based on the meta approach. Bioinformatics 24, 1344-1348, doi:10.1093/bioinformatics/btn195 (2008).

94 S. Kar, J. Fan, M. J. Smith, M. Goedert \& L. A. Amos. Repeat motifs of tau bind to the insides of microtubules in the absence of taxol. EMBO $J$ 22, 70-77, doi:10.1093/emboj/cdg001 (2003).

95 J. Biernat, N. Gustke, G. Drewes, E. M. Mandelkow \& E. Mandelkow. Phosphorylation of Ser262 strongly reduces binding of tau to microtubules: distinction between PHF-like immunoreactivity and microtubule binding. Neuron 11, 153-163 (1993). 
96 C. Ballatore, V. M. Lee \& J. Q. Trojanowski. Tau-mediated neurodegeneration in Alzheimer's disease and related disorders. Nat Rev Neurosci 8, 663-672, doi:10.1038/nrn2194 (2007).

97 G. Lee \& C. J. Leugers. Tau and tauopathies. Prog Mol Biol Transl Sci 107, 263293, doi:10.1016/B978-0-12-385883-2.00004-7 (2012).

98 T. Kampers, P. Friedhoff, J. Biernat, E. M. Mandelkow \& E. Mandelkow. RNA stimulates aggregation of microtubule-associated protein tau into Alzheimer-like paired helical filaments. FEBS Lett 399, 344-349 (1996).

99 D. R. Williams. Tauopathies: classification and clinical update on neurodegenerative diseases associated with microtubule-associated protein tau. Intern Med J 36, 652-660, doi:10.1111/j.1445-5994.2006.01153.x (2006).

100 M. Bouchard \& O. Suchowersky. Tauopathies: one disease or many? Can J Neurol Sci 38, 547-556 (2011).

101 V. M. Lee, B. J. Balin, L. Otvos, Jr. \& J. Q. Trojanowski. A68: a major subunit of paired helical filaments and derivatized forms of normal Tau. Science 251, 675-678 (1991).

102 M. Goedert, M. G. Spillantini, N. J. Cairns \& R. A. Crowther. Tau proteins of Alzheimer paired helical filaments: abnormal phosphorylation of all six brain isoforms. Neuron 8, 159-168 (1992).

103 C. L. Masters et al. Amyloid plaque core protein in Alzheimer disease and Down syndrome. Proc Natl Acad Sci U S A 82, 4245-4249 (1985).

104 M. Goedert, C. M. Wischik, R. A. Crowther, J. E. Walker \& A. Klug. Cloning and sequencing of the cDNA encoding a core protein of the paired helical filament of Alzheimer disease: identification as the microtubule-associated protein tau. Proc Natl Acad Sci U S A 85, 4051-4055 (1988).

105 H. Braak \& E. Braak. Neuropathological stageing of Alzheimer-related changes. Acta Neuropathol 82, 239-259 (1991).

106 H. Braak \& K. Del Tredici. The pathological process underlying Alzheimer's disease in individuals under thirty. Acta Neuropathol 121, 171-181, doi:10.1007/s00401-010-0789-4 (2011).

107 J. L. Price \& J. C. Morris. Tangles and plaques in nondemented aging and "preclinical" Alzheimer's disease. Ann Neurol 45, 358-368 (1999).

108 R. H. Swerdlow, J. M. Burns \& S. M. Khan. The Alzheimer's disease mitochondrial cascade hypothesis. J Alzheimers Dis 20 Suppl 2, S265-279, doi:10.3233/JAD2010-100339 (2010).

109 M. P. Mazanetz \& P. M. Fischer. Untangling tau hyperphosphorylation in drug design for neurodegenerative diseases. Nat Rev Drug Discov 6, 464-479, doi:10.1038/nrd2111 (2007).

110 G. T. Bramblett et al. Abnormal tau phosphorylation at Ser396 in Alzheimer's disease recapitulates development and contributes to reduced microtubule binding. Neuron 10, 1089-1099 (1993). 
111 H. Yoshida \& Y. Ihara. Tau in paired helical filaments is functionally distinct from fetal tau: assembly incompetence of paired helical filament-tau. J Neurochem 61, 1183-1186 (1993).

112 J. C. Augustinack, A. Schneider, E. M. Mandelkow \& B. T. Hyman. Specific tau phosphorylation sites correlate with severity of neuronal cytopathology in Alzheimer's disease. Acta Neuropathol 103, 26-35 (2002).

113 G. Drewes et al. Microtubule-associated protein/microtubule affinity-regulating kinase (p110mark). A novel protein kinase that regulates tau-microtubule interactions and dynamic instability by phosphorylation at the Alzheimer-specific site serine 262. J Biol Chem 270, 7679-7688 (1995).

114 S. Oddo et al. Triple-transgenic model of Alzheimer's disease with plaques and tangles: intracellular Abeta and synaptic dysfunction. Neuron 39, 409-421 (2003).

115 C. A. Dickey et al. The high-affinity HSP90-CHIP complex recognizes and selectively degrades phosphorylated tau client proteins. J Clin Invest 117, 648-658, doi:10.1172/JCI29715 (2007).

116 A. Schneider, J. Biernat, M. von Bergen, E. Mandelkow \& E. M. Mandelkow. Phosphorylation that detaches tau protein from microtubules (Ser262, Ser214) also protects it against aggregation into Alzheimer paired helical filaments. Biochemistry 38, 3549-3558, doi:10.1021/bi981874p (1999).

117 A. Schneider \& E. Mandelkow. Tau-based treatment strategies in neurodegenerative diseases. Neurotherapeutics 5, 443-457, doi:10.1016/j.nurt.2008.05.006 (2008).

118 M. Nangaku et al. KIF1B, a novel microtubule plus end-directed monomeric motor protein for transport of mitochondria. Cell 79, 1209-1220 (1994).

119 M. Collot, D. Louvard \& S. J. Singer. Lysosomes are associated with microtubules and not with intermediate filaments in cultured fibroblasts. Proc Natl Acad Sci U S A 81, 788-792 (1984).

120 C. Bancher et al. Accumulation of abnormally phosphorylated tau precedes the formation of neurofibrillary tangles in Alzheimer's disease. Brain Res 477, 90-99 (1989).

121 T. J. Cohen et al. The acetylation of tau inhibits its function and promotes pathological tau aggregation. Nat Commun 2, 252, doi:10.1038/ncomms 1255 (2011).

122 C. X. Gong, F. Liu, I. Grundke-Iqbal \& K. Iqbal. Post-translational modifications of tau protein in Alzheimer's disease. J Neural Transm 112, 813-838, doi:10.1007/s00702-004-0221-0 (2005).

123 G. Basurto-Islas et al. Accumulation of aspartic acid421- and glutamic acid391cleaved tau in neurofibrillary tangles correlates with progression in Alzheimer disease. $J$ Neuropathol Exp Neurol 67, 470-483, doi:10.1097/NEN.0b013e31817275c7 (2008). 
124 P. M. Horowitz et al. Early N-terminal changes and caspase-6 cleavage of tau in Alzheimer's disease. J Neurosci 24, 7895-7902, doi:10.1523/JNEUROSCI.198804.2004 (2004).

125 R. Mena, P. C. Edwards, C. R. Harrington, E. B. Mukaetova-Ladinska \& C. M. Wischik. Staging the pathological assembly of truncated tau protein into paired helical filaments in Alzheimer's disease. Acta Neuropathol 91, 633-641 (1996).

126 T. F. Gendron \& L. Petrucelli. The role of tau in neurodegeneration. Mol Neurodegener 4, 13, doi:10.1186/1750-1326-4-13 (2009).

127 S. Mondragon-Rodriguez et al. Cleavage and conformational changes of tau protein follow phosphorylation during Alzheimer's disease. Int J Exp Pathol 89, 81-90, doi:10.1111/j.1365-2613.2007.00568.x (2008).

128 M. Saito et al. Tau phosphorylation and cleavage in ethanol-induced neurodegeneration in the developing mouse brain. Neurochem Res 35, 651-659, doi:10.1007/s11064-009-0116-4 (2010).

129 T. T. Rohn et al. Caspase-9 activation and caspase cleavage of tau in the Alzheimer's disease brain. Neurobiol Dis 11, 341-354 (2002).

130 P. Poorkaj et al. Frequency of tau gene mutations in familial and sporadic cases of non-Alzheimer dementia. Arch Neurol 58, 383-387 (2001).

131 M. Hasegawa, M. J. Smith \& M. Goedert. Tau proteins with FTDP-17 mutations have a reduced ability to promote microtubule assembly. FEBS Lett 437, 207-210 (1998).

132 M. Hong et al. Mutation-specific functional impairments in distinct tau isoforms of hereditary FTDP-17. Science 282, 1914-1917 (1998).

133 M. Goedert, R. Jakes \& R. A. Crowther. Effects of frontotemporal dementia FTDP17 mutations on heparin-induced assembly of tau filaments. FEBS Lett 450, 306311 (1999).

134 S. Barghorn et al. Structure, microtubule interactions, and paired helical filament aggregation by tau mutants of frontotemporal dementias. Biochemistry 39, 1171411721 (2000).

135 L. N. Clark et al. Pathogenic implications of mutations in the tau gene in pallidoponto-nigral degeneration and related neurodegenerative disorders linked to chromosome 17. Proc Natl Acad Sci U S A 95, 13103-13107 (1998).

136 G. W. Small et al. PET of brain amyloid and tau in mild cognitive impairment. $N$ Engl J Med 355, 2652-2663, doi:10.1056/NEJMoa054625 (2006).

137 M. Kidd. Paired helical filaments in electron microscopy of Alzheimer's disease. Nature 197, 192-193 (1963).

138 R. A. Crowther \& C. M. Wischik. Image reconstruction of the Alzheimer paired helical filament. EMBO J 4, 3661-3665 (1985).

139 A. Probst, M. Tolnay, D. Langui, M. Goedert \& M. G. Spillantini. Pick's disease: hyperphosphorylated tau protein segregates to the somatoaxonal compartment. Acta Neuropathol 92, 588-596 (1996). 
140 M. G. Spillantini et al. Familial multiple system tauopathy with presenile dementia: a disease with abundant neuronal and glial tau filaments. Proc Natl Acad Sci U S A 94, 4113-4118 (1997).

141 C. N. Chirita \& J. Kuret. Evidence for an intermediate in tau filament formation. Biochemistry 43, 1704-1714, doi:10.1021/bi036034b (2004).

142 S. Khatoon, I. Grundke-Iqbal \& K. Iqbal. Brain levels of microtubule-associated protein tau are elevated in Alzheimer's disease: a radioimmuno-slot-blot assay for nanograms of the protein. J Neurochem 59, 750-753 (1992).

143 S. Barghorn, P. Davies \& E. Mandelkow. Tau paired helical filaments from Alzheimer's disease brain and assembled in vitro are based on beta-structure in the core domain. Biochemistry 43, 1694-1703, doi:10.1021/bi0357006 (2004).

144 R. Skrabana, J. Sevcik \& M. Novak. Intrinsically disordered proteins in the neurodegenerative processes: formation of tau protein paired helical filaments and their analysis. Cell Mol Neurobiol 26, 1085-1097, doi:10.1007/s10571-006-90833 (2006).

145 M. Novak, J. Kabat \& C. M. Wischik. Molecular characterization of the minimal protease resistant tau unit of the Alzheimer's disease paired helical filament. EMBO J 12, 365-370 (1993).

146 F. Chiti \& C. M. Dobson. Protein misfolding, functional amyloid, and human disease. Annu Rev Biochem 333-366, doi:10.1146/annurev.biochem.75.101304.123901 (2006).

147 D. Eisenberg \& M. Jucker. The amyloid state of proteins in human diseases. Cell 148, 1188-1203, doi:10.1016/j.cell.2012.02.022 (2012).

148 T. P. Knowles et al. Role of intermolecular forces in defining material properties of protein nanofibrils. Science 318, 1900-1903, doi:10.1126/science.1150057 (2007).

149 F. U. Hartl, A. Bracher \& M. Hayer-Hartl. Molecular chaperones in protein folding and proteostasis. Nature 475, 324-332, doi:10.1038/nature10317 (2011).

150 A. W. Fitzpatrick et al. Atomic structure and hierarchical assembly of a cross-beta amyloid fibril. Proc Natl Acad Sci $U S A$ 110, 5468-5473, doi:10.1073/pnas.1219476110 (2013).

151 L. C. Walker, M. I. Diamond, K. E. Duff \& B. T. Hyman. Mechanisms of protein seeding in neurodegenerative diseases. JAMA Neurol 70, 304-310, doi:10.1001/jamaneurol.2013.1453 (2013).

152 C. M. Wischik et al. Isolation of a fragment of tau derived from the core of the paired helical filament of Alzheimer disease. Proc Natl Acad Sci U S A 85, 45064510 (1988).

153 D. M. Wilson \& L. I. Binder. Free fatty acids stimulate the polymerization of tau and amyloid beta peptides. In vitro evidence for a common effector of pathogenesis in Alzheimer's disease. Am J Pathol 150, 2181-2195 (1997). 
154 M. Goedert et al. Assembly of microtubule-associated protein tau into Alzheimerlike filaments induced by sulphated glycosaminoglycans. Nature 383, 550-553, doi:10.1038/383550a0 (1996).

155 M. Perez, J. M. Valpuesta, M. Medina, E. Montejo de Garcini \& J. Avila. Polymerization of tau into filaments in the presence of heparin: the minimal sequence required for tau-tau interaction. J Neurochem 67, 1183-1190 (1996).

156 E. E. Congdon et al. Nucleation-dependent tau filament formation: the importance of dimerization and an estimation of elementary rate constants. J Biol Chem 283, 13806-13816, doi:10.1074/jbc.M800247200 (2008).

157 J. Berriman et al. Tau filaments from human brain and from in vitro assembly of recombinant protein show cross-beta structure. Proc Natl Acad Sci U S A 100, 9034-9038, doi:10.1073/pnas.1530287100 (2003).

158 M. von Bergen et al. Assembly of tau protein into Alzheimer paired helical filaments depends on a local sequence motif ((306)VQIVYK(311)) forming beta structure. Proc Natl Acad Sci U S A 97, 5129-5134 (2000).

159 S. Wegmann, I. D. Medalsy, E. Mandelkow \& D. J. Muller. The fuzzy coat of pathological human Tau fibrils is a two-layered polyelectrolyte brush. Proc Natl Acad Sci U S A 110, E313-321, doi:10.1073/pnas.1212100110 (2013).

160 L. Li, M. von Bergen, E. M. Mandelkow \& E. Mandelkow. Structure, stability, and aggregation of paired helical filaments from tau protein and FTDP-17 mutants probed by tryptophan scanning mutagenesis. J Biol Chem 277, 41390-41400, doi:10.1074/jbc.M206334200 (2002).

161 J. F. Smith, T. P. Knowles, C. M. Dobson, C. E. Macphee \& M. E. Welland. Characterization of the nanoscale properties of individual amyloid fibrils. Proc Natl Acad Sci U S A 103, 15806-15811, doi:10.1073/pnas.0604035103 (2006).

162 D. W. Peterson, H. Zhou, F. W. Dahlquist \& J. Lew. A soluble oligomer of tau associated with fiber formation analyzed by NMR. Biochemistry 47, 7393-7404, doi:10.1021/bi702466a (2008).

163 I. W. Hamley. Peptide fibrillization. Angew Chem Int Ed Engl 46, 8128-8147, doi:10.1002/anie.200700861 (2007).

164 C. Nishiura et al. Importance of Tyr310 residue in the third repeat of microtubule binding domain for filament formation of tau protein. J Biochem 147, 405-414, doi:10.1093/jb/mvp181 (2010).

165 W. Li \& V. M. Lee. Characterization of two VQIXXK motifs for tau fibrillization in vitro. Biochemistry 45, 15692-15701, doi:10.1021/bi061422+ (2006).

166 C. A. Lasagna-Reeves et al. Identification of oligomers at early stages of tau aggregation in Alzheimer's disease. FASEB J 26, 1946-1959, doi:10.1096/fj.11199851 (2012).

167 S. M. Ward, D. S. Himmelstein, J. K. Lancia \& L. I. Binder. Tau oligomers and tau toxicity in neurodegenerative disease. Biochem Soc Trans 40, 667-671, doi:10.1042/BST20120134 (2012). 
168 C. A. Lasagna-Reeves, D. L. Castillo-Carranza, M. J. Guerrero-Muoz, G. R. Jackson \& R. Kayed. Preparation and characterization of neurotoxic tau oligomers. Biochemistry 49, 10039-10041, doi:10.1021/bi1016233 (2010).

169 N. Zilka et al. Truncated tau from sporadic Alzheimer's disease suffices to drive neurofibrillary degeneration in vivo. FEBS Lett 580, 3582-3588, doi:10.1016/j.febslet.2006.05.029 (2006).

170 E. Sugino et al. Three-/four-repeat-dependent aggregation profile of tau microtubule-binding domain clarified by dynamic light scattering analysis. Biochem Biophys Res Commun 385, 236-240, doi:10.1016/j.bbrc.2009.05.047 (2009).

171 S. Maeda et al. Granular tau oligomers as intermediates of tau filaments. Biochemistry 46, 3856-3861, doi:10.1021/bi061359o (2007).

172 S. Maeda et al. Increased levels of granular tau oligomers: an early sign of brain aging and Alzheimer's disease. Neurosci Res 54, 197-201, doi:10.1016/j.neures.2005.11.009 (2006).

173 N. Sahara et al. Assembly of two distinct dimers and higher-order oligomers from full-length tau. Eur J Neurosci 25, 3020-3029, doi:10.1111/j.14609568.2007.05555.x (2007).

174 Y. J. Zhang, Y. F. Xu, X. Q. Chen, X. C. Wang \& J. Z. Wang. Nitration and oligomerization of tau induced by peroxynitrite inhibit its microtubule-binding activity. FEBS Lett 579, 2421-2427, doi:10.1016/j.febslet.2005.03.041 (2005).

175 N. Sahara et al. Molecular chaperone-mediated tau protein metabolism counteracts the formation of granular tau oligomers in human brain. J Neurosci Res 85, 30983108, doi:10.1002/jnr.21417 (2007).

176 M. A. Meraz-Rios, K. I. Lira-De Leon, V. Campos-Pena, M. A. De AndaHernandez \& R. Mena-Lopez. Tau oligomers and aggregation in Alzheimer's disease. J Neurochem 112, 1353-1367, doi:10.1111/j.1471-4159.2009.06511.x (2010).

177 J. Kuret et al. Evaluating triggers and enhancers of tau fibrillization. Microsc Res Tech 67, 141-155, doi:10.1002/jemt.20187 (2005).

178 P. S. Aisen, J. Cummings \& L. S. Schneider. Symptomatic and nonamyloid/tau based pharmacologic treatment for Alzheimer disease. Cold Spring Harb Perspect Med 2, a006395, doi:10.1101/cshperspect.a006395 (2012).

179 I. Churcher. Tau therapeutic strategies for the treatment of Alzheimer's disease. Curr Top Med Chem 6, 579-595 (2006).

180 J. Y. Chin et al. Microtubule-affinity regulating kinase (MARK) is tightly associated with neurofibrillary tangles in Alzheimer brain: a fluorescence resonance energy transfer study. J Neuropathol Exp Neurol 59, 966-971 (2000).

181 I. Nishimura, Y. Yang \& B. Lu. PAR-1 kinase plays an initiator role in a temporally ordered phosphorylation process that confers tau toxicity in Drosophila. Cell 116, 671-682 (2004). 
182 J. Toshima, J. Y. Toshima, K. Takeuchi, R. Mori \& K. Mizuno. Cofilin phosphorylation and actin reorganization activities of testicular protein kinase 2 and its predominant expression in testicular Sertoli cells. J Biol Chem 276, 3144931458, doi:10.1074/jbc.M102988200 (2001).

183 L. Meijer et al. Inhibition of cyclin-dependent kinases, GSK-3beta and CK1 by hymenialdisine, a marine sponge constituent. Chem Biol 7, 51-63 (2000).

184 Q.Zheng-Fischhofer et al. Sequential phosphorylation of Tau by glycogen synthase kinase-3beta and protein kinase A at Thr212 and Ser214 generates the Alzheimerspecific epitope of antibody AT100 and requires a paired-helical-filament-like conformation. Eur J Biochem 252, 542-552 (1998).

185 S. J. Liu et al. Tau becomes a more favorable substrate for GSK-3 when it is prephosphorylated by PKA in rat brain. J Biol Chem 279, 50078-50088, doi:10.1074/jbc.M406109200 (2004).

186 H. Shuntoh, N. Sakamoto, S. Matsuyama, M. Saitoh \& C. Tanaka. Molecular structure of the $\mathrm{C}$ beta catalytic subunit of rat cAMP-dependent protein kinase and differential expression of $\mathrm{C}$ alpha and $\mathrm{C}$ beta isoforms in rat tissues and cultured cells. Biochim Biophys Acta 1131, 175-180 (1992).

187 M. S. Lee et al. Neurotoxicity induces cleavage of p35 to p25 by calpain. Nature 405, 360-364, doi:10.1038/35012636 (2000).

188 G. Morfini et al. A novel CDK5-dependent pathway for regulating GSK3 activity and kinesin-driven motility in neurons. EMBO $J \mathbf{2 3}, 2235-2245$, doi:10.1038/sj.emboj.7600237 (2004).

189 J. L. Hallows, K. Chen, R. A. DePinho \& I. Vincent. Decreased cyclin-dependent kinase 5 (cdk5) activity is accompanied by redistribution of cdk5 and cytoskeletal proteins and increased cytoskeletal protein phosphorylation in p35 null mice. $J$ Neurosci 23, 10633-10644 (2003).

190 M. E. Saez, R. Ramirez-Lorca, F. J. Moron \& A. Ruiz. The therapeutic potential of the calpain family: new aspects. Drug Discov Today 11, 917-923, doi:10.1016/j.drudis.2006.08.009 (2006).

191 B. W. Doble \& J. R. Woodgett. GSK-3: tricks of the trade for a multi-tasking kinase. J Cell Sci 116, 1175-1186 (2003).

192 S. Lovestone et al. Alzheimer's disease-like phosphorylation of the microtubuleassociated protein tau by glycogen synthase kinase-3 in transfected mammalian cells. Curr Biol 4, 1077-1086 (1994).

193 F. Hernandez, J. Borrell, C. Guaza, J. Avila \& J. J. Lucas. Spatial learning deficit in transgenic mice that conditionally over-express GSK-3beta in the brain but do not form tau filaments. J Neurochem 83, 1529-1533 (2002).

194 G. Alvarez et al. Regulation of tau phosphorylation and protection against betaamyloid-induced neurodegeneration by lithium. Possible implications for Alzheimer's disease. Bipolar Disord 4, 153-165 (2002). 
195 R. Bhat et al. Structural insights and biological effects of glycogen synthase kinase 3-specific inhibitor AR-A014418. J Biol Chem 278, 45937-45945, doi:10.1074/jbc.M306268200 (2003).

196 C. J. Phiel \& P. S. Klein. Molecular targets of lithium action. Annu Rev Pharmacol Toxicol 41, 789-813, doi:10.1146/annurev.pharmtox.41.1.789 (2001).

197 L. Sun et al. Inhibition of protein phosphatase 2A- and protein phosphatase 1induced tau hyperphosphorylation and impairment of spatial memory retention in rats. Neuroscience 118, 1175-1182 (2003).

198 J. J. Pei et al. Okadaic-acid-induced inhibition of protein phosphatase 2A produces activation of mitogen-activated protein kinases ERK1/2, MEK1/2, and p70 S6, similar to that in Alzheimer's disease. Am $J$ Pathol 163, 845-858, doi:10.1016/S0002-9440(10)63445-1 (2003).

199 E. M. Sigurdsson. Tau-focused immunotherapy for Alzheimer's disease and related tauopathies. Curr Alzheimer Res 6, 446-450 (2009).

200 H. Rosenmann et al. Tauopathy-like abnormalities and neurologic deficits in mice immunized with neuronal tau protein. Arch Neurol 63, 1459-1467, doi:10.1001/archneur.63.10.1459 (2006).

201 A. A. Asuni, A. Boutajangout, D. Quartermain \& E. M. Sigurdsson. Immunotherapy targeting pathological tau conformers in a tangle mouse model reduces brain pathology with associated functional improvements. J Neurosci 27, 9115-9129, doi:10.1523/JNEUROSCI.2361-07.2007 (2007).

202 M. J. Winton et al. Intraneuronal APP, not free Abeta peptides in 3xTg-AD mice: implications for tau versus Abeta-mediated Alzheimer neurodegeneration. $J$ Neurosci 31, 7691-7699, doi:10.1523/JNEUROSCI.6637-10.2011 (2011).

203 J. M. Orgogozo et al. Subacute meningoencephalitis in a subset of patients with AD after Abeta42 immunization. Neurology 61, 46-54 (2003).

204 B. Ravikumar, R. Duden \& D. C. Rubinsztein. Aggregate-prone proteins with polyglutamine and polyalanine expansions are degraded by autophagy. Hum Mol Genet 11, 1107-1117 (2002).

205 J. L. Webb, B. Ravikumar, J. Atkins, J. N. Skepper \& D. C. Rubinsztein. AlphaSynuclein is degraded by both autophagy and the proteasome. J Biol Chem 278, 25009-25013, doi:10.1074/jbc.M300227200 (2003).

206 Z. Berger et al. Rapamycin alleviates toxicity of different aggregate-prone proteins. Hum Mol Genet 15, 433-442, doi:10.1093/hmg/ddi458 (2006).

207 D. J. Klionsky \& S. D. Emr. Autophagy as a regulated pathway of cellular degradation. Science 290, 1717-1721 (2000).

208 D. C. Rubinsztein et al. Dyneins, autophagy, aggregation and neurodegeneration. Autophagy 1, 177-178 (2005).

209 C. A. Dickey et al. HSP induction mediates selective clearance of tau phosphorylated at proline-directed Ser/Thr sites but not KXGS (MARK) sites. FASEB J 20, 753-755, doi:10.1096/fj.05-5343fje (2006). 
210 H. Shimura, Y. Miura-Shimura \& K. S. Kosik. Binding of tau to heat shock protein 27 leads to decreased concentration of hyperphosphorylated tau and enhanced cell survival. J Biol Chem 279, 17957-17962, doi:10.1074/jbc.M400351200 (2004).

211 W. Luo et al. Roles of heat-shock protein 90 in maintaining and facilitating the neurodegenerative phenotype in tauopathies. Proc Natl Acad Sci U S A 104, 95119516, doi:10.1073/pnas.0701055104 (2007).

212 S. L. Karsten et al. A genomic screen for modifiers of tauopathy identifies puromycin-sensitive aminopeptidase as an inhibitor of tau-induced neurodegeneration. Neuron 51, 549-560, doi:10.1016/j.neuron.2006.07.019 (2006).

213 L. Fasulo, G. Ugolini \& A. Cattaneo. Apoptotic effect of caspase-3 cleaved tau in hippocampal neurons and its potentiation by tau FTDP-mutation N279K. $J$ Alzheimers Dis 7, 3-13 (2005).

214 J. W. Connell et al. Quantitative analysis of tau isoform transcripts in sporadic tauopathies. Brain Res Mol Brain Res 137, 104-109, doi:10.1016/j.molbrainres.2005.02.014 (2005).

215 M. Takanashi, H. Mori, K. Arima, Y. Mizuno \& N. Hattori. Expression patterns of tau mRNA isoforms correlate with susceptible lesions in progressive supranuclear palsy and corticobasal degeneration. Brain Res Mol Brain Res 104, 210-219 (2002).

216 A. Boutajangout, A. Boom, K. Leroy \& J. P. Brion. Expression of tau mRNA and soluble tau isoforms in affected and non-affected brain areas in Alzheimer's disease. FEBS Lett 576, 183-189, doi:10.1016/j.febslet.2004.09.011 (2004).

217 A. Seitz et al. Single-molecule investigation of the interference between kinesin, tau and MAP2c. EMBO J 21, 4896-4905 (2002).

218 T. Rodriguez-Martin et al. Reprogramming of tau alternative splicing by spliceosome-mediated RNA trans-splicing: implications for tauopathies. Proc Natl Acad Sci U S A 102, 15659-15664, doi:10.1073/pnas.0503150102 (2005).

219 C. Ballatore et al. Microtubule stabilizing agents as potential treatment for Alzheimer's disease and related neurodegenerative tauopathies. J Med Chem 55, 8979-8996, doi:10.1021/jm301079z (2012).

220 O. A. Shemesh \& M. E. Spira. Rescue of neurons from undergoing hallmark tauinduced Alzheimer's disease cell pathologies by the antimitotic drug paclitaxel. Neurobiol Dis 43, 163-175, doi:10.1016/j.nbd.2011.03.008 (2011).

221 V. Das \& J. H. Miller. Microtubule stabilization by peloruside A and paclitaxel rescues degenerating neurons from okadaic acid-induced tau phosphorylation. Eur J Neurosci 35, 1705-1717, doi:10.1111/j.1460-9568.2012.08084.x (2012).

222 T. Ishihara et al. Attenuated neurodegenerative disease phenotype in tau transgenic mouse lacking neurofilaments. J Neurosci 21, 6026-6035 (2001).

223 B. Zhang et al. Microtubule-binding drugs offset tau sequestration by stabilizing microtubules and reversing fast axonal transport deficits in a tauopathy model. Proc Natl Acad Sci U S A 102, 227-231, doi:10.1073/pnas.0406361102 (2005). 
224 D. M. Bollag et al. Epothilones, a new class of microtubule-stabilizing agents with a taxol-like mechanism of action. Cancer Res 55, 2325-2333 (1995).

225 C. A. Lipinski. Drug-like properties and the causes of poor solubility and poor permeability. J Pharmacol Toxicol Methods 44, 235-249 (2000).

226 J. Inglese et al. High-throughput screening assays for the identification of chemical probes. Nat Chem Biol 3, 466-479, doi:10.1038/nchembio.2007.17 (2007).

227 P. J. Hajduk \& J. Greer. A decade of fragment-based drug design: strategic advances and lessons learned. Nat Rev Drug Discov 6, 211-219, doi: $10.1038 / \mathrm{nrd} 2220$ (2007).

228 K. A. Giuliano, Y. T. Chen \& D. L. Taylor. High-content screening with siRNA optimizes a cell biological approach to drug discovery: defining the role of P53 activation in the cellular response to anticancer drugs. J Biomol Screen 9, 557-568, doi:10.1177/1087057104265387 (2004).

229 J. Klages, M. Coles \& H. Kessler. NMR-based screening: a powerful tool in fragment-based drug discovery. The Analyst 132, 692, doi:10.1039/b709658p (2007).

230 R. Khurana et al. Mechanism of thioflavin T binding to amyloid fibrils. $J$ Struct Biol 151, 229-238, doi:10.1016/j.jsb.2005.06.006 (2005).

231 S. Taniguchi et al. Inhibition of heparin-induced tau filament formation by phenothiazines, polyphenols, and porphyrins. J Biol Chem 280, 7614-7623, doi:10.1074/jbc.M408714200 (2005).

232 M. Pickhardt et al. Anthraquinones inhibit tau aggregation and dissolve Alzheimer's paired helical filaments in vitro and in cells. J Biol Chem 280, 36283635, doi:10.1074/jbc.M410984200 (2005).

233 A. Crowe, C. Ballatore, E. Hyde, J. Q. Trojanowski \& V. M. Lee. High throughput screening for small molecule inhibitors of heparin-induced tau fibril formation. Biochem Biophys Res Commun 358, 1-6, doi:10.1016/j.bbrc.2007.03.056 (2007).

234 R. J. Castellani, H. G. Lee, X. Zhu, G. Perry \& M. A. Smith. Alzheimer disease pathology as a host response. J Neuropathol Exp Neurol 67, 523-531, doi:10.1097/NEN.0b013e318177eaf4 (2008).

235 J. W. Park et al. Amyloid fibrillar meshwork formation of iron-induced oligomeric species of Abeta40 with phthalocyanine tetrasulfonate and its toxic consequences. Chembiochem 9, 2602-2605, doi:10.1002/cbic.200800343 (2008).

236 K. Murakami et al. Synthesis, aggregation, neurotoxicity, and secondary structure of various A beta 1-42 mutants of familial Alzheimer's disease at positions 21-23. Biochem Biophys Res Commun 294, 5-10, doi:10.1016/S0006-291X(02)00430-8 (2002).

237 B. Y. Feng et al. Small-molecule aggregates inhibit amyloid polymerization. Nat Chem Biol 4, 197-199, doi:10.1038/nchembio.65 (2008).

238 B. Bulic et al. Rhodanine-based tau aggregation inhibitors in cell models of tauopathy. Angew Chem Int Ed Engl 46, 9215-9219, doi:10.1002/anie.200704051 (2007). 
239 B. Bulic et al. Development of tau aggregation inhibitors for Alzheimer's disease. Angew Chem Int Ed Engl 48, 1740-1752, doi:10.1002/anie.200802621 (2009).

240 B. Bulic, M. Pickhardt \& E. Mandelkow. Progress and developments in tau aggregation inhibitors for Alzheimer disease. J Med Chem 56, 4135-4155, doi:10.1021/jm3017317 (2013).

241 J. M. Ford, J. M. Yang \& W. N. Hait. P-glycoprotein-mediated multidrug resistance: experimental and clinical strategies for its reversal. Cancer Treat Res 87, 3-38 (1996).

242 N. Motohashi, M. Kawase, K. Satoh \& H. Sakagami. Cytotoxic potential of phenothiazines. Curr Drug Targets 7, 1055-1066 (2006).

243 K. Pluta et al. Anticancer activity of newly synthesized azaphenothiazines from NCI's anticancer screening bank. Pharmacol Rep 62, 319-332 (2010).

244 S. J. Peroutka \& S. H. Synder. Relationship of neuroleptic drug effects at brain dopamine, serotonin, alpha-adrenergic, and histamine receptors to clinical potency. Am J Psychiatry 137, 1518-1522 (1980).

245 A. Schotte et al. Risperidone compared with new and reference antipsychotic drugs: in vitro and in vivo receptor binding. Psychopharmacology (Berl) 124, 57-73 (1996).

246 S. H. Snyder, S. P. Banerjee, H. I. Yamamura \& D. Greenberg. Drugs, neurotransmitters, and schizophrenia. Science 184, 1243-1253, doi:10.1126/science.184.4143.1243 (1974).

247 A. Seelig, R. Gottschlich \& R. M. Devant. A method to determine the ability of drugs to diffuse through the blood-brain barrier. Proc Natl Acad Sci U S A 91, 6872 (1994).

248 A. Jaszczyszyn et al. Chemical structure of phenothiazines and their biological activity. Pharmacol Rep 64, 16-23 (2012).

249 M. Wainwright \& L. Amaral. The phenothiazinium chromophore and the evolution of antimalarial drugs. Trop Med Int Health 10, 501-511, doi:10.1111/j.13653156.2005.01417.x (2005).

250 M. A. Ramirez \& N. L. Borja. Epalrestat: an aldose reductase inhibitor for the treatment of diabetic neuropathy. Pharmacotherapy 28, 646-655, doi:10.1592/phco.28.5.646 (2008).

251 M. Pickhardt et al. Phenylthiazolyl-hydrazide and its derivatives are potent inhibitors of tau aggregation and toxicity in vitro and in cells. Biochemistry 46, 10016-10023, doi:10.1021/bi700878g (2007).

252 G. Larbig, M. Pickhardt, D. G. Lloyd, B. Schmidt \& E. Mandelkow. Screening for inhibitors of tau protein aggregation into Alzheimer paired helical filaments: a ligand based approach results in successful scaffold hopping. Curr Alzheimer Res 4, 315-323 (2007).

253 M. Pickhardt et al. N-phenylamine derivatives as aggregation inhibitors in cell models of tauopathy. Curr Alzheimer Res 4, 397-402 (2007). 
254 D. E. Ehrnhoefer et al. EGCG redirects amyloidogenic polypeptides into unstructured, off-pathway oligomers. Nat Struct Mol Biol 15, 558-566, doi:10.1038/nsmb.1437 (2008).

255 S. A. Mandel et al. Multifunctional activities of green tea catechins in neuroprotection. Modulation of cell survival genes, iron-dependent oxidative stress and PKC signaling pathway. Neurosignals 14, 46-60, doi:10.1159/000085385 (2005).

256 N. Khan, F. Afaq, M. Saleem, N. Ahmad \& H. Mukhtar. Targeting multiple signaling pathways by green tea polyphenol (-)-epigallocatechin-3-gallate. Cancer Res 66, 2500-2505, doi:10.1158/0008-5472.CAN-05-3636 (2006).

257 Y. Sun, W. C. Hung, F. Y. Chen, C. C. Lee \& H. W. Huang. Interaction of tea catechin (-)-epigallocatechin gallate with lipid bilayers. Biophys $J$ 96, 1026-1035, doi:10.1016/j.bpj.2008.11.007 (2009).

258 H. Y. Kim et al. Structural properties of pore-forming oligomers of alphasynuclein. J Am Chem Soc 131, 17482-17489, doi:10.1021/ja9077599 (2009).

259 J. Bieschke et al. EGCG remodels mature alpha-synuclein and amyloid-beta fibrils and reduces cellular toxicity. Proc Natl Acad Sci U S A 107, 7710-7715, doi:10.1073/pnas.0910723107 (2010).

260 K. Rezai-Zadeh et al. Green tea epigallocatechin-3-gallate (EGCG) modulates amyloid precursor protein cleavage and reduces cerebral amyloidosis in Alzheimer transgenic mice. J Neurosci 25, 8807-8814, doi:10.1523/JNEUROSCI.152105.2005 (2005).

261 K. Rezai-Zadeh et al. Green tea epigallocatechin-3-gallate (EGCG) reduces betaamyloid mediated cognitive impairment and modulates tau pathology in Alzheimer transgenic mice. Brain Res 1214, 177-187, doi:10.1016/j.brainres.2008.02.107 (2008).

262 D. E. Ehrnhoefer. Green tea (-)-epigallocatechin-gallate modulates early events in huntingtin misfolding and reduces toxicity in Huntington's disease models. Human Molecular Genetics 15, 2743-2751, doi:10.1093/hmg/ddl210 (2006).

263 S. M. Henning et al. Bioavailability and antioxidant activity of tea flavanols after consumption of green tea, black tea, or a green tea extract supplement. Am J Clin Nutr 80, 1558-1564 (2004).

264 N. Zhu et al. Identification of oxidation products of (-)-epigallocatechin gallate and (-)-epigallocatechin with $\mathrm{H}(2) \mathrm{O}(2)$. J Agric Food Chem 48, 979-981 (2000).

265 B. E. Roberts \& J. Shorter. Escaping amyloid fate. Nat Struct Mol Biol 15, 544546, doi:10.1038/nsmb0608-544 (2008).

266 C. Chirita, M. Necula \& J. Kuret. Ligand-dependent inhibition and reversal of tau filament formation. Biochemistry 43, 2879-2887, doi:10.1021/bi036094h (2004).

267 E. E. Congdon, M. Necula, R. D. Blackstone \& J. Kuret. Potency of a tau fibrillization inhibitor is influenced by its aggregation state. Arch Biochem Biophys 465, 127-135, doi:10.1016/j.abb.2007.05.004 (2007). 
268 H. Hermel, W. Schmahl \& H. Mohwald. Selective staining by the fluorochrome, 5,5-diphenyl-9-ethyl-DiOC2(3). I. Physicochemical studies of dye-dye and dyetissue interactions. Biotech Histochem 74, 221-228 (1999).

269 N. S. Honson, J. R. Jensen, M. V. Darby \& J. Kuret. Potent inhibition of tau fibrillization with a multivalent ligand. Biochem Biophys Res Commun 363, 229234, doi:10.1016/j.bbrc.2007.08.166 (2007).

270 K. N. Schafer et al. Structure-activity relationship of cyclic thiacarbocyanine tau aggregation inhibitors. Bioorg Med Chem Lett 21, 3273-3276, doi:10.1016/j.bmcl.2011.04.039 (2011).

271 A. Crowe et al. Identification of aminothienopyridazine inhibitors of tau assembly by quantitative high-throughput screening. Biochemistry 48, 7732-7745, doi:10.1021/bi9006435 (2009).

272 C. Ballatore et al. Discovery of brain-penetrant, orally bioavailable aminothienopyridazine inhibitors of tau aggregation. J Med Chem 53, 3739-3747, doi:10.1021/jm100138f (2010).

273 A. Crowe et al. Aminothienopyridazines and methylene blue affect Tau fibrillization via cysteine oxidation. J Biol Chem 288, 11024-11037, doi:10.1074/jbc.M112.436006 (2013).

274 K. Kassab, D. A. El Fadeel \& M. Fadel. Topical photodynamic therapy using transfersomal aluminum phthalocyanine tetrasulfonate: in vitro and in vivo study. Lasers Med Sci, doi:10.1007/s10103-012-1256-3 (2013).

275 W. S. Medina, N. A. dos Santos, C. Curti, A. C. Tedesco \& A. C. dos Santos. Effects of zinc phthalocyanine tetrasulfonate-based photodynamic therapy on rat brain isolated mitochondria. Chem Biol Interact 179, 402-406 (2009).

276 R. Linstead. J. Chem. Soc 1016 (1934).

277 W. S. Caughey et al. Cyclic tetrapyrrole sulfonation, metals, and oligomerization in antiprion activity. Antimicrob Agents Chemother 51, 3887-3894, doi:10.1128/AAC.01599-06 (2007).

278 A. Snow. Phthalocyanine Aggregation. The Porphyrin Handbook 17, 130-175 (2003).

279 J. P. Zelina et al. Influence of surfactant-based microheterogeneous fluids on aggregation of copper phthalocyanine tetrasulfonate. Journal of Porphyrins and Phthalocyanines 3, 188-195, doi:Doi 10.1002/(Sici)10991409(199903)3:3<188::Aid-Jpp122>3.0.Co;2-A (1999).

280 W. S. Caughey, L. D. Raymond, M. Horiuchi \& B. Caughey. Inhibition of proteaseresistant prion protein formation by porphyrins and phthalocyanines. Proc Natl Acad Sci U S A 95, 12117-12122 (1998).

281 S. A. Priola, A. Raines \& W. Caughey. Prophylactic and therapeutic effects of phthalocyanine tetrasulfonate in scrapie-infected mice. J Infect Dis 188, 699-705, doi:10.1086/377310 (2003).

282 S. A. Priola. Porphyrin and Phthalocyanine Antiscrapie Compounds. Science 287, 1503-1506, doi:10.1126/science.287.5457.1503 (2000). 
283 D. R. Dee et al. Phthalocyanine tetrasulfonates bind to multiple sites on nativelyfolded prion protein. Biochim Biophys Acta 1824, 826-832, doi:10.1016/j.bbapap.2012.03.011 (2012).

284 E. N. Lee et al. Phthalocyanine tetrasulfonates affect the amyloid formation and cytotoxicity of alpha-synuclein. Biochemistry 43, 3704-3715, doi:10.1021/bi0356707 (2004).

285 G. R. Lamberto et al. Structural and mechanistic basis behind the inhibitory interaction of PcTS on alpha-synuclein amyloid fibril formation. Proc Natl Acad Sci U S A 106, 21057-21062, doi:10.1073/pnas.0902603106 (2009).

286 C. M. Wischik, P. C. Edwards, R. Y. Lai, M. Roth \& C. R. Harrington. Selective inhibition of Alzheimer disease-like tau aggregation by phenothiazines. Proc Natl Acad Sci U S A 93, 11213-11218 (1996).

287 M. Havelcova, P. Kubat \& I. Nemcova. Photophysical properties of thiazine dyes in aqueous solution and in micelles. Dyes and Pigments 44, 49-54, doi:Doi 10.1016/S0143-7208(99)00070-4 (1999).

288 A. K. Bruchey \& F. Gonzalez-Lima. Behavioral, Physiological and Biochemical Hormetic Responses to the Autoxidizable Dye Methylene Blue. Am J Pharmacol Toxicol 3, $72-79$ (2008).

289 Y. Yusim, D. Livingstone \& A. Sidi. Blue dyes, blue people: the systemic effects of blue dyes when administered via different routes. J Clin Anesth 19, 315-321, doi:10.1016/j.jclinane.2007.01.006 (2007).

290 G. E. Burrows. Methylene blue: effects and disposition in sheep. J Vet Pharmacol Ther 7, 225-231 (1984).

291 E. Akoury et al. Mechanistic basis of phenothiazine-driven inhibition of Tau aggregation. Angew Chem Int Ed Engl 52, 3511-3515, doi:10.1002/anie.201208290 (2013).

292 J. Luna-Munoz et al. Thiazin red as a neuropathological tool for the rapid diagnosis of Alzheimer's disease in tissue imprints. Acta Neuropathologica 116, 507-515, doi:DOI 10.1007/s00401-008-0431-x (2008).

293 I. Walter-Sack et al. High absolute bioavailability of methylene blue given as an aqueous oral formulation. Eur J Clin Pharmacol 65, 179-189, doi:10.1007/s00228008-0563-x (2009).

294 A. Warth et al. Turquoise to dark green organs at autopsy. Virchows Arch 454, 341344, doi:10.1007/s00428-009-0734-x (2009).

295 H. Atamna \& R. Kumar. Protective role of methylene blue in Alzheimer's disease via mitochondria and cytochrome c oxidase. J Alzheimers Dis 20 Suppl 2, S439452, doi:10.3233/JAD-2010-100414 (2010).

296 A. M. Wang et al. Inhibition of hsp70 by methylene blue affects signaling protein function and ubiquitination and modulates polyglutamine protein degradation. $J$ Biol Chem 285, 15714-15723, doi:10.1074/jbc.M109.098806 (2010). 
297 U. K. Jinwal et al. Chemical manipulation of hsp70 ATPase activity regulates tau stability. J Neurosci 29, 12079-12088, doi:10.1523/JNEUROSCI.3345-09.2009 (2009).

298 F. van Bebber, D. Paquet, A. Hruscha, B. Schmid \& C. Haass. Methylene blue fails to inhibit Tau and polyglutamine protein dependent toxicity in zebrafish. Neurobiol Dis 39, 265-271, doi:10.1016/j.nbd.2010.03.023 (2010).

299 C. Wischik. Press release announcing the initiation of a global phase 3 clinical trial in a type of Frontotemporal Dementia (FTD) also known as Pick's Disease. $J$ .Nutr.Health Aging 13, 367-369 (2012).

300 P. Cavaliere et al. Binding of methylene blue to a surface cleft inhibits the oligomerization and fibrillization of prion protein. Biochim Biophys Acta 1832, 2028, doi:10.1016/j.bbadis.2012.09.005 (2013).

301 M. Necula et al. Methylene blue inhibits amyloid Abeta oligomerization by promoting fibrillization. Biochemistry 46, 8850-8860, doi:10.1021/bi700411k (2007).

302 J. M. Chalker, G. J. Bernardes, Y. A. Lin \& B. G. Davis. Chemical modification of proteins at cysteine: opportunities in chemistry and biology. Chem Asian J 4, 630640, doi:10.1002/asia.200800427 (2009).

303 C. Jacob, G. I. Giles, N. M. Giles \& H. Sies. Sulfur and selenium: the role of oxidation state in protein structure and function. Angew Chem Int Ed Engl 42, 47424758, doi:10.1002/anie.200300573 (2003).

304 K. Bhattacharya, K. B. Rank, D. B. Evans \& S. K. Sharma. Role of cysteine-291 and cysteine-322 in the polymerization of human tau into Alzheimer-like filaments. Biochem Biophys Res Commun 285, 20-26, doi:10.1006/bbrc.2001.5116 (2001).

305 O. Schweers, E. M. Mandelkow, J. Biernat \& E. Mandelkow. Oxidation of cysteine-322 in the repeat domain of microtubule-associated protein tau controls the in vitro assembly of paired helical filaments. Proc Natl Acad Sci U S A 92, 8463-8467 (1995).

306 Y. Furukawa, K. Kaneko \& N. Nukina. Tau protein assembles into isoform- and disulfide-dependent polymorphic fibrils with distinct structural properties. J Biol Chem 286, 27236-27246, doi:10.1074/jbc.M111.248963 (2011).

307 N. J. Kettenhofen \& M. J. Wood. Formation, reactivity, and detection of protein sulfenic acids. Chem Res Toxicol 23, 1633-1646, doi:10.1021/tx100237w (2010).

308 J. M. Denu \& K. G. Tanner. Specific and reversible inactivation of protein tyrosine phosphatases by hydrogen peroxide: evidence for a sulfenic acid intermediate and implications for redox regulation. Biochemistry 37, 5633-5642, doi:10.1021/bi973035t (1998).

309 Z. A. Wood, E. Schroder, J. R. Harris \& L. B. Poole. Structure, mechanism and regulation of peroxiredoxins. Trends in Biochemical Sciences 28, 32-40, doi:Pii S0968-0004(02)00003-8

Doi 10.1016/S0968-0004(02)00003-8 (2003). 
310 A. Pihl \& R. Lange. Interaction of Oxidized Glutathione, Cystamine Monosulfoxide, and Tetrathionate with --Sh Groups of Rabbit Muscle DGlyceraldehyde 3-Phosphate Dehydrogenase. Journal of Biological Chemistry 237, $1356-\&$ (1962).

311 A. N. Glazer \& E. L. Smith. Sulfur Distribution of Papain. Journal of Biological Chemistry 240, 201-\& (1965).

312 E. G. Demaster, B. J. Quast, B. Redfern \& H. T. Nagasawa. Reaction of NitricOxide with the Free Sulfhydryl-Group of Human Serum-Albumin Yields a Sulfenic Acid and Nitrous-Oxide. Biochemistry 34, 11494-11499, doi:Doi 10.1021/Bi00036a023 (1995).

313 Y. Miyata et al. Cysteine reactivity distinguishes redox sensing by the heatinducible and constitutive forms of heat shock protein 70. Chem Biol 19, 13911399, doi:10.1016/j.chembiol.2012.07.026 (2012).

314 T. Laufen et al. Mechanism of regulation of hsp70 chaperones by DnaJ cochaperones. Proc Natl Acad Sci U S A 96, 5452-5457 (1999).

315 P. Walsh, D. Bursac, Y. C. Law, D. Cyr \& T. Lithgow. The J-protein family: modulating protein assembly, disassembly and translocation. EMBO Rep 5, 567571, doi:10.1038/sj.embor.7400172 (2004).

316 J. C. Young, I. Moarefi \& F. U. Hartl. Hsp90: a specialized but essential proteinfolding tool. J Cell Biol 154, 267-273 (2001).

317 R. K. R et al. HSPIR: a manually annotated heat shock protein information resource. Bioinformatics 28, 2853-2855, doi:10.1093/bioinformatics/bts520 (2012).

318 K. R. Patterson et al. Heat shock protein 70 prevents both tau aggregation and the inhibitory effects of preexisting tau aggregates on fast axonal transport. Biochemistry 50, 10300-10310, doi:10.1021/bi2009147 (2011).

319 K. Voss, B. Combs, K. R. Patterson, L. I. Binder \& T. C. Gamblin. Hsp70 alters tau function and aggregation in an isoform specific manner. Biochemistry 51, 888898, doi:10.1021/bi2018078 (2012).

320 Y. Kato et al. HSP70 and HSP90 Differentially Regulate Translocation of Extracellular Antigen to the Cytosol for Cross-Presentation. Autoimmune Dis 2012, 745962, doi:10.1155/2012/745962 (2012).

$321 \mathrm{~J}$. F. Abisambra et al. Exploiting the diversity of the heat-shock protein family for primary and secondary tauopathy therapeutics. Curr Neuropharmacol 9, 623-631, doi:10.2174/157015911798376226 (2011).

322 A. D. Thompson et al. Analysis of the tau-associated proteome reveals that exchange of Hsp70 for Hsp90 is involved in tau degradation. ACS Chem Biol 7, 1677-1686, doi:10.1021/cb3002599 (2012).

323 S. B. Goldfarb et al. Differential effects of Hsc70 and Hsp70 on the intracellular trafficking and functional expression of epithelial sodium channels. Proc Natl Acad Sci U S A 103, 5817-5822, doi:10.1073/pnas.0507903103 (2006). 
324 U. K. Jinwal et al. Imbalance of Hsp70 family variants fosters tau accumulation. FASEB J 27, 1450-1459, doi:10.1096/fj.12-220889 (2013).

325 S. Spera, M. Ikura \& A. Bax. Measurement of the exchange rates of rapidly exchanging amide protons: application to the study of calmodulin and its complex with a myosin light chain kinase fragment. J Biomol NMR 1, 155-165 (1991).

326 F. A. Mulder \& M. Filatov. NMR chemical shift data and ab initio shielding calculations: emerging tools for protein structure determination. Chem Soc Rev 39, 578-590, doi:10.1039/b811366c (2010).

327 S. Schwarzinger et al. Sequence-dependent correction of random coil NMR chemical shifts. J Am Chem Soc 123, 2970-2978 (2001).

328 D. G. Donne et al. Structure of the recombinant full-length hamster prion protein PrP(29-231): the N terminus is highly flexible. Proc Natl Acad Sci U S A 94, 1345213457 (1997).

329 D. Eliezer, E. Kutluay, R. Bussell, Jr. \& G. Browne. Conformational properties of alpha-synuclein in its free and lipid-associated states. J Mol Biol 307, 1061-1073, doi:10.1006/jmbi.2001.4538 (2001).

330 C. W. Bertoncini et al. Structural characterization of the intrinsically unfolded protein beta-synuclein, a natural negative regulator of alpha-synuclein aggregation. J Mol Biol 372, 708-722, doi:10.1016/j.jmb.2007.07.009 (2007).

331 J. Keeler. Understanding NMR spectroscopy. 2nd edn, (John Wiley and Sons, 2010).

332 J. Cavanagh. Protein NMR spectroscopy : principles and practice. 2nd edn, (Academic Press, 2007).

333 J. Yao, H. J. Dyson \& P. E. Wright. Chemical shift dispersion and secondary structure prediction in unfolded and partly folded proteins. FEBS Lett 419, 285-289 (1997).

334 J. R. Gillespie \& D. Shortle. Characterization of long-range structure in the denatured state of staphylococcal nuclease. II. Distance restraints from paramagnetic relaxation and calculation of an ensemble of structures. $\mathrm{J} \mathrm{Mol} \mathrm{Biol}$ 268, 170-184, doi:10.1006/jmbi.1997.0953 (1997).

335 P. A. Kosen. Spin labeling of proteins. Methods Enzymol 177, 86-121 (1989).

336 J. L. Battiste \& G. Wagner. Utilization of site-directed spin labeling and highresolution heteronuclear nuclear magnetic resonance for global fold determination of large proteins with limited nuclear overhauser effect data. Biochemistry 39, 5355-5365 (2000).

337 V. J. McParland, A. P. Kalverda, S. W. Homans \& S. E. Radford. Structural properties of an amyloid precursor of beta(2)-microglobulin. Nat Struct Biol 9, 326331, doi:10.1038/nsb791 (2002).

338 D. K. Wilkins et al. Hydrodynamic radii of native and denatured proteins measured by pulse field gradient NMR techniques. Biochemistry 38, 16424-16431 (1999). 
339 C. S. J. Jr. Diffusion ordered nuclear magnetic resonance spectroscopy: principles and applications. Progress in Nuclear Magnetic Resonance Spectroscopy 34, 203256 (1999).

340 G. Zheng, T. Stait-Gardner, P. G. Anil Kumar, A. M. Torres \& W. S. Price. PGSTEWATERGATE: an STE-based PGSE NMR sequence with excellent solvent suppression. J Magn Reson 191, 159-163, doi:10.1016/j.jmr.2007.12.001 (2008).

341 D. Some. Light-scattering-based analysis of biomolecular interactions. Biophys Rev 5, 147-158, doi:10.1007/s12551-013-0107-1 (2013).

342 G. Zandomeneghi, M. R. Krebs, M. G. McCammon \& M. Fandrich. FTIR reveals structural differences between native beta-sheet proteins and amyloid fibrils. Protein Sci 13, 3314-3321, doi:10.1110/ps.041024904 (2004).

343 L. M. Miller, M. W. Bourassa \& R. J. Smith. FTIR spectroscopic imaging of protein aggregation in living cells. Biochim Biophys Acta 1828, 2339-2346, doi:10.1016/j.bbamem.2013.01.014 (2013).

344 N. J. Greenfield. Using circular dichroism spectra to estimate protein secondary structure. Nat Protoc 1, 2876-2890, doi:10.1038/nprot.2006.202 (2006).

345 H. D. Mertens \& D. I. Svergun. Structural characterization of proteins and complexes using small-angle X-ray solution scattering. J Struct Biol 172, 128-141, doi:10.1016/j.jsb.2010.06.012 (2010).

346 F. Spinozzi et al. Quaternary structure heterogeneity of oligomeric proteins: a SAXS and SANS study of the dissociation products of Octopus vulgaris hemocyanin. PLoS One 7, e49644, doi:10.1371/journal.pone.0049644 (2012).

347 F. Prischi et al. Structural bases for the interaction of frataxin with the central components of iron-sulphur cluster assembly. Nat Commun 1, 95, doi:10.1038/ncomms1097 (2010).

348 H. Liu \& P. H. Zwart. Determining pair distance distribution function from SAXS data using parametric functionals. $J$ Struct Biol 180, 226-234, doi:10.1016/j.jsb.2012.05.011 (2012).

349 M. V. Petoukhov \& D. I. Svergun. Global rigid body modeling of macromolecular complexes against small-angle scattering data. Biophys J 89, 1237-1250, doi:10.1529/biophysj.105.064154 (2005).

350 P. Bernado \& D. I. Svergun. Analysis of intrinsically disordered proteins by smallangle X-ray scattering. Methods Mol Biol 896, 107-122, doi:10.1007/978-1-46143704-8_7 (2012). 


\section{Inhibition of Tau Filament Formation by}

\section{Conformational Modulation}

Elias Akoury ${ }^{\mathrm{a}}$, Michal Gajda ${ }^{\mathrm{a}}$, Marcus Pickhardt ${ }^{\mathrm{b}}$, Jacek Biernat ${ }^{\mathrm{b}}$, Pornsuwan Soraya $^{\mathrm{d}}$, Christian Griesinger ${ }^{\mathrm{a}}$, Eckhard Mandelkow ${ }^{\mathrm{b}, \mathrm{c}}$, Markus Zweckstetter ${ }^{\mathrm{a}, \mathrm{e}}$

a Department for NMR-based Structural Biology, Max Planck Institute for Biophysical Chemistry, 37077 Göttingen, Germany. ${ }^{\mathrm{b}}$ German Center for Neurodegenerative Diseases (DZNE), LudwigErhard-Allee 2, 53175 Bonn, Germany. ${ }^{\mathrm{c}}$ CAESAR Research Center, Ludwig-Erhard-Allee 2, 53175 Bonn, Germany, ${ }^{d}$ RG Electron Spin Resonance Spectroscopy, Max Planck Institute for

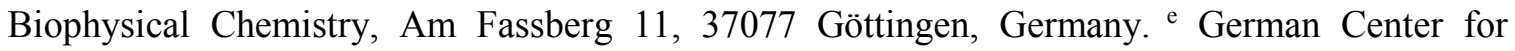
Neurodegenerative Diseases (DZNE), Göttingen, Germany.

* E. Akoury performed and analyzed NMR, DLS, CD and FTIR experiments and wrote the manuscript. 


\title{
2. Inhibition of Tau Filament Formation by Conformational Modulation
}

\begin{abstract}
Anti-aggregation drugs play an important role in therapeutic approaches for Alzheimer's disease. Although, a large number of small molecules that inhibit the aggregation of the Tau protein have been identified, little is known about their mode of action. Here we reveal the mechanism and the nature of Tau species that are generated by interaction of Tau with the organic compound Phthalocyanine tetrasulfonate (PcTS). We demonstrate that PcTS interferes with Tau filament formation by targeting the protein into soluble oligomers. A combination of NMR spectroscopy, electron paramagnetic resonance and small-angle X-ray scattering reveals that the soluble Tau oligomers contain a dynamic, non-cooperatively stabilized core with a diameter of 30 to $40 \mathrm{~nm}$ that is distinct from the core of Tau filaments. Our results suggest that specific modulation of the conformation of Tau is a viable strategy for reduction of pathogenic Tau deposits.
\end{abstract}

\section{INTRODUCTION}

Neurodegenerative diseases share related pathological processes characterized by the generation of proteinaceous deposits exhibiting excessive $\beta$-sheet structures. ${ }^{1}$ Growing evidence has implicated the aggregates in the onset, progression and clinical symptoms of these disorders. ${ }^{2}$ The most widespread dementia syndrome is Alzheimer's Disease (AD), which is characterized by the progressive accumulation of extracellular senile plaques consisting of $\beta$-amyloid polypeptide and intracellular neurofibrillary tangles consisting of Tau protein. ${ }^{3,4}$

Tau protein belongs to the class of "natively unfolded" or intrinsically disordered proteins. ${ }^{5,6}$ It is abundant in neuronal axons and interacts with tubulin to stabilize and 


\section{Inhibition of Tau Filament Formation by Conformational Modulation}

promote microtubule assembly for the transport of vesicles and organelles. ${ }^{7}$ In solution, Tau is highly dynamic with an intricate domain structure. ${ }^{8}$ During the course of AD progression, Tau becomes excessively phosphorylated, loses its function and aggregates into neurofibrillary tangles. ${ }^{4}$ The accumulation of Tau aggregates is a multistep process that involves the formation of various transient species. Increasing evidence suggests that small oligomeric species contribute to Tau-mediated neurotoxicity. ${ }^{9}$

So far only symptomatic treatment is available for $\mathrm{AD}$ and other protein misfolding diseases and new therapeutic concepts range from Tau vaccinations 10 and antiphosphorylation strategies ${ }^{11}$ to microtubule-stabilizing ${ }^{12}$ and anti-aggregation drugs. ${ }^{13-22}$ One important class of aggregation inhibitors are porphyrins such as Phthalocyanine tetrasulfonate (PcTS). ${ }^{16,22,23}$ PcTS has been widely investigated for its in vivo prophylactic and therapeutic effects in scrapie disease and inhibits the formation of protease-resistant prion protein aggregates. ${ }^{23,24}$ In addition, PcTS can interfere with Tau aggregation and is able to disassemble Tau filaments. ${ }^{16}$ Taking advantage of its chelating and molecular self-assembly properties, PcTS was also employed to remove the redoxactive metals that induce toxic $A \beta_{40}$ oligomeric species and to convert the aggregates into an amyloid fibrillar meshwork. ${ }^{25}$

Despite the wealth of potential inhibitors of Tau aggregation little is known about the mechanisms of inhibition and the nature of the generated Tau species. Here we study the interaction of PcTS with 441-residue human Tau protein (Figure S1). Using a combination of NMR spectroscopy, electron paramagnetic resonance (EPR) and smallangle X-ray scattering (SAXS) we reveal detailed insights into the mechanisms of Tau aggregation inhibition and the structure and dynamics of soluble Tau oligomers. 


\section{Inhibition of Tau Filament Formation by Conformational Modulation}

\section{EXPERIMENTAL SECTION}

Proteins and Reagents. Unlabeled and labeled wild-type and mutated human Tau protein isoforms and constructs (htau40, htau24, htau23, K18) were expressed and purified as described previously. ${ }^{26}$ Briefly, proteins were expressed in the vector pNG2 (a derivative of pET-3a; Merck) in Escherichia coli strain BL21 (DE3). The expressed proteins were purified from bacterial extracts by making use of the heat stability of the protein and by FPLC SP-Sepharose chromatography (GE Healthcare). To label uniformly the Tau proteins with ${ }^{15} \mathrm{~N}$ and ${ }^{13} \mathrm{C}$ isotopes, E.coli bacteria were grown in minimal medium containing $1 \mathrm{~g} /$ liter of ${ }^{15} \mathrm{NH}_{4} \mathrm{Cl}$ and $4 \mathrm{~g} /$ liter of D-Glucose $\left({ }^{13} \mathrm{C}-6\right)$, or $1 \mathrm{~g} /$ liter of ${ }^{15} \mathrm{NH}_{4} \mathrm{Cl}$ alone. The cell pellets were resuspended in extraction buffer (50 mM MES, $500 \mathrm{mM} \mathrm{NaCl}$, $1 \mathrm{mM} \mathrm{MgCl}_{2}, 1 \mathrm{mM}$ EGTA, $5 \mathrm{mM}$ DTT, pH 6.8) complemented with protease inhibitor mix, disrupted with a French press and boiled for $20 \mathrm{~min}$. The soluble extract was isolated by centrifugation, and the supernatant was dialyzed against buffer A (20 mM MES, $50 \mathrm{mM}$ $\mathrm{NaCl}, 1 \mathrm{mM}$ EGTA, $1 \mathrm{mM} \mathrm{MgCl} 2,2$ mM DTT, 0,1 mM PMSF, pH 6.8) and loaded on an FPLC SP -Sepharose column. The proteins were eluted by a gel filtration column by a linear gradient of buffer B (20 mM MES, $1 \mathrm{M} \mathrm{NaCl}, 1 \mathrm{mM}$ EGTA, $1 \mathrm{mM} \mathrm{MgCl} 2,2 \mathrm{mM}$ DTT, $0.1 \mathrm{mM}$ PMSF, pH 6.8). The Tau isoforms were separated from breakdown products by a gel filtration column Superdex G-200 (GE Healthcare).

PcTS was purchased from MP Biomedicals (MP Biomedicals S.A. Heidelberg, Germany). ThS, urea and GuSCN were purschased from Sigma (Sigma-Aldrich Chemie $\mathrm{GmbH}$, Schnelldorf, Germany). Spin-labeling of Tau was performed as described previously. $^{8}$

Aggregation assay. $50 \mu \mathrm{M}$ monomeric htau40 was incubated with $12.5 \mu \mathrm{M}$ heparin in $20 \mathrm{mM}$ BES-buffer, $\mathrm{pH} 7.4$ and $1 \mathrm{mM}$ DTT for 10 minutes at $95^{\circ} \mathrm{C}$ to destroy 


\section{Inhibition of Tau Filament Formation by Conformational Modulation}

the intra-molecular disulfide bridges of compact monomers. After cooling down the reaction mixture to $37^{\circ} \mathrm{C}$ the sample was replenished with $1 \mathrm{mM}$ DTT again and a protease inhibitor-mix (1 mM PMSF, $1 \mathrm{mM}$ EDTA, $1 \mathrm{mM}$ EGTA, $1 \mu \mathrm{g} / \mathrm{mL}$ leupeptin, $1 \mu \mathrm{g} / \mathrm{mL}$ aprotinin, and $1 \mu \mathrm{g} / \mathrm{ml}$ pepstatin) was added. The resulting solution was separated into different vials. PcTS was added to the vials in concentrations ranging from $200 \mathrm{pM}$ to 200 $\mu \mathrm{M}$ and incubated for 10 days at $37{ }^{\circ} \mathrm{C} .1 \mathrm{mM}$ DTT was added daily to avoid its depletion. Before each fluorescence measurement the samples were equilibrated 30 minutes at room temperature and diluted 6-fold with BES buffer followed by the addition of $20 \mu \mathrm{M}$ ThS. Fluorescence was measured on a Safire (TECAN) fluorimeter with excitation and emission wavelengths of $440 \mathrm{~nm}$ and $521 \mathrm{~nm}$, respectively. In addition, a $200 \mu \mathrm{M}$ PcTS solution was prepared in the same buffer conditions to examine the absorption properties in the range of 250 to $700 \mathrm{~nm}$ and to exclude possible interference with $\mathrm{ThS}$ fluorescence.

SDS-page analysis. For SDS-page analysis $50 \mu \mathrm{M}$ monomeric htau40 samples containing $1 \mathrm{mM}$ DTT were prepared in $50 \mathrm{mM}$ PBS buffer, $\mathrm{pH} 7.4$, and heated for 20 minutes at $95{ }^{\circ} \mathrm{C}$. After cooling down in a water bath, the samples were incubated with 750 $\mu \mathrm{M}$ PcTS for 1 or 24 hours at 4 or $37{ }^{\circ} \mathrm{C}$. Reference (Ferritin $440 \mathrm{kDa}$ and protein marker) and control samples (htau40 in PBS buffers) were prepared and all samples were run on 10 $\%$ SDS-PAGE gels. Two additional htau40 samples were prepared in PBS and incubated for 3 days at $37{ }^{\circ} \mathrm{C}$ in the presence or absence of PcTS before running them on a $10 \%$ SDS-PAGE gel.

Spin Labeling of Tau. We constructed and labeled with (1-oxy-2,2,5,5tetramethyl-D-pyrroline-3-methyl)-methanethiosulfonate (MTSL) the double cysteines of the wild type (C291 and C322) as well as the mutants containing a single cysteine (C291A and C291A/C322G at different sites A15C, A72C, A125C, A178C, A239C, S352C, 


\section{Inhibition of Tau Filament Formation by Conformational Modulation}

A384C, and S416C) using the following protocol: DTT was first removed from the buffer prior labeling using size exclusion chromatography (PD-10 columns, GE Healthcare, Freiburg, Germany); then the monomeric protein was equilibrated in phosphate buffer saline (PBS) buffer pH 7.4. MTSL (5-fold molar excess in ethyl acetate) was allowed to react with the free sulfhydryl group(s) of the cysteine(s) at $21{ }^{0} \mathrm{C}$ for 2.5 hours. The unreacted spin label was removed by PD-10 columns equilibrated in $50 \mathrm{mM}$ Na phosphate buffer $\mathrm{pH} 6.8$ and the resulting spin-labeled protein was concentrated by using Amicon Ultra-15 (molecular weight cutoff 3000, Millipore, Cork, Ireland).

NMR spectroscopy. NMR experiments were recorded at $5{ }^{\circ} \mathrm{C}$ on Bruker Avance $700 \mathrm{MHz}$ or $800 \mathrm{MHz}$ spectrometers equipped with cryogenic probes. NMR samples contained ${ }^{15} \mathrm{~N} /{ }^{13} \mathrm{C}$ double labeled or ${ }^{15} \mathrm{~N}$ single labeled protein in $50 \mathrm{mM}$ phosphate buffer $\mathrm{pH}$ 6.8. $1 \mathrm{mM}$ DTT and $10 \%(\mathrm{v} / \mathrm{v}) \mathrm{D}_{2} \mathrm{O}$. Different concentrations of PcTS were prepared in the same phosphate buffer and the dilution factors were corrected whenever needed. For each measurement (except when time dependence was investigated) PcTS was freshly added to a sample of monomeric Tau protein and the measurement was started within less than 15 minutes. In case of PcTS titrations, increasing concentrations of PcTS were subsequently added to the same Tau sample with a delay of less than 10 minutes between individual HSQC measurements. In order to probe the time dependence on NMR signal intensities the samples were kept at room temperature for the specified time periods (3 days and 7 days, Figure S3c; 2 weeks, Figure S3d).

Interaction of PcTS with $100 \mu \mathrm{M}$ Tau was investigated using two-dimensional ${ }^{1} \mathrm{H}-$

${ }^{15} \mathrm{~N}$ HSQC spectra recorded with 600 complex points and 32 scans per increment with spectral widths of $8389 \mathrm{~Hz}$ and $1844 \mathrm{~Hz}$ in the ${ }^{1} \mathrm{H}$ and ${ }^{15} \mathrm{~N}$ dimensions, respectively. The total measurement time for each ${ }^{1} \mathrm{H}-{ }^{15} \mathrm{~N}$ HSQC was six hours. In case of the control 


\section{Inhibition of Tau Filament Formation by Conformational Modulation}

titration with $10 \mu \mathrm{M}$ htau40 (Figure S6), the recording time for a single ${ }^{1} \mathrm{H}-{ }^{15} \mathrm{~N}$ HSQC was increased to 20 hours. NMR intensity ratio plots were reported with a 3-residues averaging window. Two dimensional ${ }^{1} \mathrm{H}-{ }^{13} \mathrm{C}$ HSQC spectra were acquired using 1024 complex points and 12 scans per increment with spectral widths of $7003 \mathrm{~Hz}$ and $12330 \mathrm{~Hz}$ (aliphatic region) or $5282 \mathrm{~Hz}$ (aromatic region) in the ${ }^{1} \mathrm{H}$ and ${ }^{13} \mathrm{C}$ dimensions, respectively, resulting in a total measurement time of five hours. Spectra were processed with NMRPipe ${ }^{27}$ and analyzed using the software Sparky3.

For equilibrium denaturation experiments a solution of PcTS and ${ }^{15} \mathrm{~N}$-labeled htau40 $(200 \mu \mathrm{M})$ in a ratio of 15 to 1 was prepared in $50 \mathrm{mM}$ phosphate buffer $\mathrm{pH} 6.8$, containing $1.8 \mathrm{mM}$ DTT and $10 \%(\mathrm{v} / \mathrm{v}) \mathrm{D}_{2} \mathrm{O}$. A $2 \mathrm{D}{ }^{1} \mathrm{H}-{ }^{15} \mathrm{~N}$ HSQC spectrum was measured for three hours. Then the denaturant concentration was increased and at each denaturant concentration a $2 \mathrm{D}{ }^{1} \mathrm{H}-{ }^{15} \mathrm{~N}$ HSQC spectrum (measurement time: three hours) was recorded. Denaturants solutions were prepared immediately before use and concentrations ranged from 0 to $8 \mathrm{M}$ for urea and 0 to $3 \mathrm{M}$ for GuSCN. NMR signal intensities were fitted to sigmoid, Hill, or exponential curve functions using Igor Pro 6.22A.

PRE broadening was investigated using ${ }^{15} \mathrm{~N}$-labeled htau40 at a concentration of 15 $\mu M$. PRE effects were measured from the peak intensity ratios between two $2 \mathrm{D}{ }^{1} \mathrm{H}-{ }^{15} \mathrm{~N}$ HSQC NMR spectra acquired in the presence of the nitroxide radical and after addition of $4 \mathrm{mM}$ DTT (heated to $45^{\circ} \mathrm{C}$ for $10 \mathrm{~min}$ before measurement) to the same sample. Addition of DTT will cleave the MTSL tag from the cysteine residue, such that the spin label is no longer attached to the protein and the protein is in the diamagnetic state. The measurement time for each $2 \mathrm{D}{ }^{1} \mathrm{H}-{ }^{15} \mathrm{~N}$ HSQC NMR was 17 hours.

NMR diffusion experiments were recorded on Bruker Avance $600 \mathrm{MHz}$ spectrometer equipped with cryogenic probe for htau40 protein $(40 \mu \mathrm{M}$ with or without 


\section{Inhibition of Tau Filament Formation by Conformational Modulation}

15-folded excess PcTS) using a stimulated-echo based pulsed gradient spin-echo sequence incorporating the WATERGATE solvent suppression. ${ }^{28}$ The diffusion time and the length of the gradient pulses were optimized for each sample. The gradient strength was linearly increased from $2 \%$ to $95 \%$ of the maximum gradient strength in 30 steps, where the $100 \%$ gradient strength corresponds to $55.14 \mathrm{G} / \mathrm{cm}$. Each ${ }^{1} \mathrm{H}$ spectrum was recorded with 128 scans and 16K complex over a spectral width of $8992 \mathrm{~Hz}$, resulting in a total experimental time of two hours for a complete NMR diffusion experiment. Several peaks in the aliphatic region of htau40 were selected and the intensities of their diffusionbased spin-echo attenuation were extracted to determine an average diffusion coefficient. Note that the NMR-based characterization of the Tau oligomers occurs via their flexible N- and C-terminal tails (Figure 2) From the apparent diffusion coefficients of htau40 and a range of protein standards (cytochrome C 12.4KDa, 17.8 ̊; lysozyme 14.3 KDa, $20.5 \AA$; myoglobin $18 \mathrm{KDa}, 21.2 \AA$; and ovalbumin $45 \mathrm{KDa}, 30.5 \AA$ ) the Stokes radii were calculated. ${ }^{29}$

EPR spectroscopy. Selected single-labeled htau40 $(20 \mu \mathrm{M}$ in phosphate buffer $\mathrm{pH}$ 6.8) samples were freshly prepared in absence or presence of 15-fold excess PcTS and transferred in 1-mm inner diameter capillary prior each measurement. Continuous waveEPR spectra were recorded at room temperature at X-band on a Bruker Elexys 500 spectrometer equipped with an ER 4122SHQE resonator. The incident power used was at $2.0 \mathrm{~mW}$ over a field range of $100 \mathrm{G}$ and 1024 spectral points, and a modulation amplitude of $0.8 \mathrm{G}$. The line broadening of the central peak of each spin-labeled htau 40 was quantified using the width of the peak-to-peak separation. Integration of the EPR spectra in the absence and presence of PcTS resulted in similar double integral values, indicating that the 


\section{Inhibition of Tau Filament Formation by Conformational Modulation}

same amount of Tau protein contributes to the visible EPR signal in the absence and presence of PcTS.

Fourier transform-infrared spectroscopy. FTIR spectra of $50 \mu \mathrm{M}$ K18 and 100 $\mu \mathrm{M}$ htau40 (with and without $1.5 \mathrm{mM}$ PcTS) in $50 \mathrm{mM}$ phosphate buffer were collected at room temperature using the Bruker Confocheck FT-IR spectrometer. The delay time between preparation of the Tau-PcTS sample and start of the FT-IR measurements was about 10 minutes. The FT-IR setup consists of the AquaSpec transmission cell designed for measurements of aqueous solutions and equipped with a cryogenic MCT detector cooled with liquid nitrogen. Reported spectra represent averages of 5 scans recorded between $3000 \mathrm{~cm}^{-1}$ and $1000 \mathrm{~cm}^{-1}$ where each scan was acquired with a spectral resolution of $4 \mathrm{~cm}^{-1}$ and measurement time of 100 seconds. The same measurement was repeated three times using freshly prepared samples to confirm the reproducibility of the results.

Dynamic Light Scattering. DLS measurements were performed at $5{ }^{\circ} \mathrm{C}$ using a DynaPro Titan temperature controlled microsampler (Wyatt Technologies Corporation). Samples $(40 \mu \mathrm{M}$ of htau40 in $50 \mathrm{mM}$ phosphate buffer, $\mathrm{pH} 6.8$ and $1 \mathrm{mM}$ DTT inserted in $50 \mu$ flow cells in absence or presence of 15-fold excess of PcTS) were illuminated by a $25 \mathrm{~mW}, 780 \mathrm{~nm}$ solid-state laser, and the intensity of $90^{\circ}$ angle scattered light was measured at $4 \mu \mathrm{s}$ intervals by a solid-state avalanche photodiode. Measurements were performed using freshly prepared samples, each experiment lasted 20 minutes and was repeated 6 times to confirm the reproducibility of the results (i.e. total of 2 hours). The average values with their standard deviations were analyzed using the software package Dynamics 6.7.7.9.

Circular Dichroism. CD spectra of $30 \mu \mathrm{M}$ K18 and $10 \mu \mathrm{M}$ htau40 in $50 \mathrm{mM}$ phosphate buffer were acquired at room temperature using a Chirascan CD spectrometer 


\section{Inhibition of Tau Filament Formation by Conformational Modulation}

(Applied Photophysics Limited) in the absence or presence of 15-fold excess of PcTS. Spectra were acquired with $350 \mu \mathrm{l}$ in a cuvette with $1-\mathrm{mm}$ path cell over the range covering 190 to $250 \mathrm{~nm}$ using a $1 \mathrm{~nm}$ band width and a scanning speed of $20 \mathrm{~nm} / \mathrm{min}$. Five scans (measurement time for a single scan was 10 minutes) were averaged for each dataset and the subsequent spectra of the buffer constituents were substracted from the protein samples. The same measurement was repeated at three times using freshly prepared samples to confirm the reproducibility of the results. Data are expressed in terms of the mean residual ellipticity $(\theta)$ in $\operatorname{deg} \mathrm{cm}^{2} \mathrm{dmol}^{-1}$.

Electron microscopy. The sample with the highest PcTS concentration (PcTS:htau40, $200 \mu \mathrm{M}$ to $50 \mu \mathrm{M}$ ) from the aggregation assays was diluted 1:7.5 with 20 mM BES buffer, pH 7.4 and $5 \mu$ were placed on copper 600 mesh grids covered with carbonfilm. The sample was stained with $2 \%$ uranyl acetate after immunostaining with goldlabeled anti-tau antibody K9JA-gold ${ }_{10 \mathrm{~nm}}$ and examined on a CM12 tranmission electron microscope.

Fluorescence-activated cell sorting. The assay was performed with inducible N2a cells expressing the four-repeat-domain construct with a single deletion at position 280

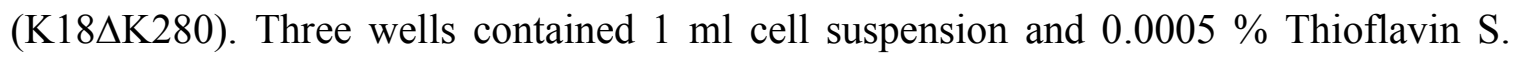
Negative controls were not treated further. Positive controls were treated with $1 \mu \mathrm{g} / \mathrm{ml}$ doxycyclin. Samples were treated by addition of $50 \mu \mathrm{M}$ PcTS and the same amount of

doxycyclin. After incubation for 4 days at $37{ }^{\circ} \mathrm{C}$, the floating and adherent cells were combined, pelleted for 5 minutes (at $295 \mathrm{~g}$ ) and washed once with PBS buffer. Cells were counted in a BD FACS Canto TMII flow cytometry system. The cell distribution was determined by accessing the cell size from the forward scatter FSC and the cell granularity 


\section{Inhibition of Tau Filament Formation by Conformational Modulation}

from the side scatter SSC. Cells with ThS-positive aggregates were measured by the fluorescent signal intensity in the FTIC channel $\left(\lambda_{\text {excitation }}=495 \mathrm{~nm}, \lambda_{\text {emission }}=519 \mathrm{~nm}\right)$.

Small Angle X-ray Scattering Data. Data were collected at X33 at the European Molecular Biology Laboratory on DORIS III (DESY) at a wavelength of $1.5 \AA$ at $25^{\circ} \mathrm{C}$ using a Pilatus $1 \mathrm{M}$ photon counting detector. Samples were freshly prepared immediately prior to the SAXS measurements and contained Tau concentrations from $2.5 \mathrm{mg} / \mathrm{ml}$ to 10 $\mathrm{mg} / \mathrm{ml}$ and $1.8 \mathrm{mM}$ DTT in $50 \mathrm{mM}$ phosphate buffer $\mathrm{pH}$ 6.8. At 15-fold excess of PcTS, data were measured before and after filtering using centrifugal filter with $0.2 \mu \mathrm{m}$ cutoff (Millipore, Carrigtwohill, Ireland). Each sample was exposed to 8 frames of 15 seconds each, with consistency checks between frames. Data analysis was performed in ATSAS $2.3^{30}$. The average molecular masses of solutes were estimated from the intensity extrapolated to zero angle. The maximum diameter was estimated by indirect fourier transform using ATSAS $2.3^{30}$. The amount of monomeric htau40 at intermediate PcTS concentrations was estimated using NMR spectroscopy (as described in Supporting Information). Estimation of the average number of Tau molecules in soluble oligomers at intermediate PcTS:Tau ratios using a combination of NMR and SAXS. At 10-fold and 15fold excess of PcTS residues G120-S400 were broadened beyond detection, demonstrating that Tau has been completely converted into soluble oligomers. Thus, the SAXS signal for these samples can exclusively be attributed to Tau oligomers. In contrast, at intermediate PcTS concentrations both monomeric and oligomeric Tau species were present (according to NMR and SDS-PAGE) complicating the SAXS analysis. Nevertheless to allow a detailed analysis of the oligomeric Tau species at these PcTS concentrations, we took advantage of NMR spectroscopy. Assuming that a similar region of Tau is part of the oligomers formed at high and intermediate PcTS concentrations, an assumption that is 


\section{Inhibition of Tau Filament Formation by Conformational Modulation}

supported by a comparison of the NMR broadening profiles at 5 -fold and 15 -fold excess of PcTS (Figure 2a), the NMR signal intensity ratios of the residues in this region were averaged and used as estimate for the amount of residual monomeric Tau. The average oligomeric number $n$ of the remaining fraction was then computed from the nominal concentration of Tau, the percentage $r$ of remaining monomer in solution (as estimated by NMR) and the absolute scattering intensity $I(0)$ divided by the intensity for monomeric Tau $I_{\text {monomeric }}(0)$ :

$$
n=\frac{\frac{I(0)}{I_{\text {monomeric }}(0)}-r}{1-r}
$$

Errors in the oligomeric number were estimated by taking the minimum and maximum of the following:

$$
n(e I(0), e r)=\frac{\frac{I(0) \pm e I(0)}{I_{\text {monomeric }}(0)}-r \mp e r}{1-r \mp e r}
$$

where $e r$ is the error for the monomer ratio (two times the standard deviation of relative NMR intensities in the region G120-S400), and $e I(0)$ the error of the total scattering intensity, estimated to fall within one standard deviation of scattering intensities at different protein concentrations, respectively. Within the given range of values $(I(0) \gg$ 1.0 and $(1.0>r \pm e r>0.02)$ it is thus highly probable that $n$ lies within the limits;

$$
\frac{\frac{I(0)+e I(0)}{I_{\text {monomeric }}(0)}-r-e r}{1-r-e r} \geq n \geq \frac{\frac{I(0)-e I(0)}{I_{\text {monomeric }}(0)}-r+e r}{1-r+e r}
$$




\section{Inhibition of Tau Filament Formation by Conformational Modulation}

\section{RESULTS}

Proteins PcTS Inhibits Tau Filament Formation. The aggregation of the Tau protein in vitro is promoted by polyanions such as heparin. ${ }^{31}$ Using this approach, we tested the ability of PcTS (Figure 1a) to suppress fibrillization of htau40. Porphyrins do not interfere with the binding of Tau to heparin excluding a competitive effect. ${ }^{16}$ PcTS decreased the amount of fibrillized Tau as quantified by thioflavinS (ThS) in a dosedependent manner (Figure 1b). From the assay, we determined an IC50 value of $1.25 \mu \mathrm{M}$. For the 412-residue isoform htau34 an IC50 value of $67 \mu \mathrm{M}$ was previously determined suggesting isoform-specific differences. ${ }^{16}$ Using electron microscopy we then investigated the morphology of htau40 that was incubated for 10 days at $37{ }^{0} \mathrm{C}$ in the absence and presence of a 4-fold molar excess of PcTS. In the absence of PcTS, we observed formation of Tau filaments with the characteristic twisted appearance (Figure 1c). In contrast, globular particles and other amorphous aggregates were detected in the presence of PcTS (Figure 1d and Figure S2). Sodium dodecyl sulfate polyacrylamide gel electrophoresis (SDS-PAGE) revealed predominantly two high molecular weight Tau species with approximate molecular weights of 300 and $450 \mathrm{kDa}$ (Figure 1e and Figure S3). In addition, time-dependent changes in the distribution of high molecular weight species showed that the $450 \mathrm{kDa}$ species becomes predominant with increasing incubation time (Figure S3a). 


\section{Inhibition of Tau Filament Formation by Conformational Modulation}

a

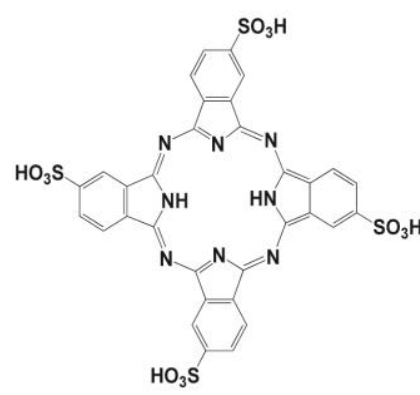

C

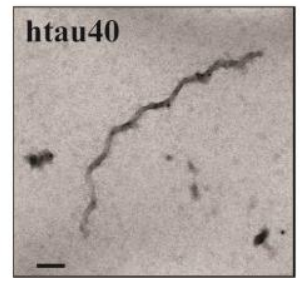

b

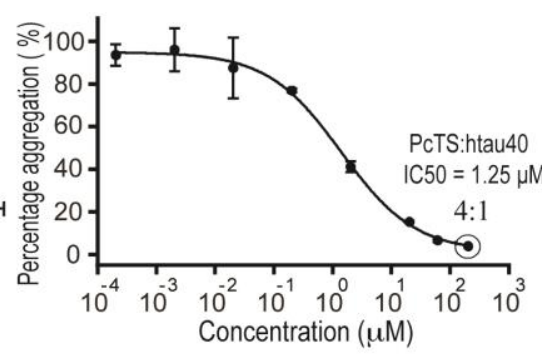

d

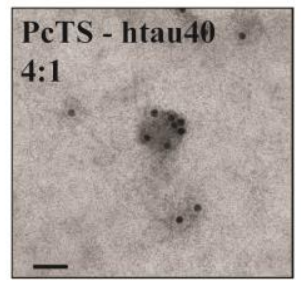

e

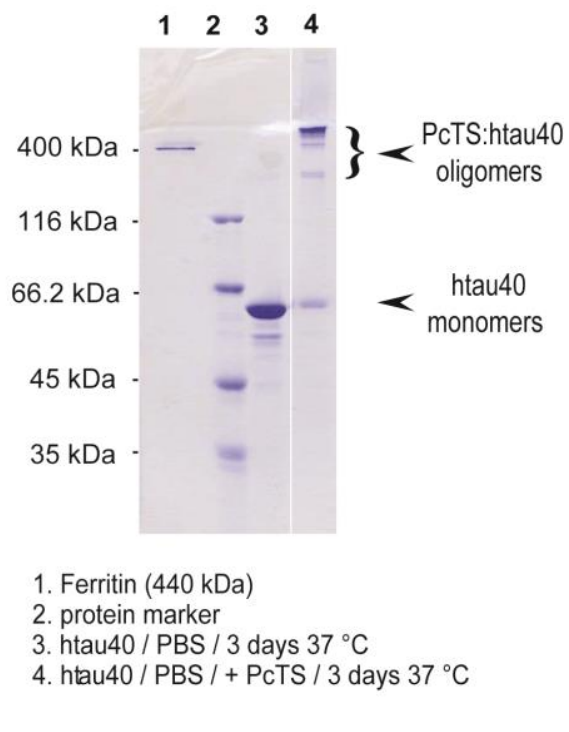

Figure 1: PcTS inhibits Tau filament formation by induction of oligomers.

(a) Chemical structure of PcTS. (b) ThS fluorescence after incubation of $50 \mu \mathrm{M}$ htau 40 wild-type with 12.5 $\mu \mathrm{M}$ heparin for $10 \mathrm{~min}$ at $95^{\circ} \mathrm{C}$ and subsequent incubation for 10 days at $37{ }^{\circ} \mathrm{C}$ in the presence of increasing concentrations ( $200 \mathrm{pM}-200 \mu \mathrm{M})$ of PcTS. Electron micrographs of (c) heparin-induced htau40 aggregates showing PHF-like structures (scale bar, $100 \mathrm{~nm}$ ) and (d) the sample treated with 4-fold molar excess of PcTS (scale bar, $40 \mathrm{~nm}$ ). The black dots represent the gold-labeled antibody K9JA-gold ${ }_{10 \mathrm{~nm}}$ used for immunostaining. (e) SDS-PAGE analysis of PcTS-stimulated htau40 oligomers.

The Interaction with PcTS is Mediated by Aromatic Rings. To obtain insight into the mechanism of PcTS-induced oligomerization of Tau, we probed the PcTS:Tau interaction using NMR spectroscopy. Upon addition of PcTS, the intensities of several peaks in $2 \mathrm{D}{ }^{1} \mathrm{H}^{15} \mathrm{~N}$ heteronuclear single quantum coherence (HSQC) spectra of htau40 decreased in a concentration-dependent manner (Figure 2a). The broadening is caused by an exchange of Tau molecules between the free and the PcTS-bound state. At equimolar concentration of PcTS, the aromatic residues Y310, F346 and F378, which are located in the repeats R3-R4 and the $\mathrm{C}$-terminal region, were most strongly affected (Figures 2a, 2b). 


\section{Inhibition of Tau Filament Formation by Conformational Modulation}

In addition, Y197 in the proline-rich region P2 and Y394 bound to PcTS. Only weak changes in NMR signal position and intensity were observed for the other three aromatic residues of htau40 (F8, Y18, Y29) at the Tau N-terminus. The importance of aromatic residues was further supported by interaction studies performed for the Tau mutant htau40/Y310N: ${ }^{306}$ VQIVNK ${ }^{311}$ showed reduced chemical shift and intensity changes upon addition of PcTS (Figure 2c). Instead, stronger changes were observed for Y197, F346 and F378 indicative of an enhanced interaction. At 5:1 and 15:1 PcTS:htau40/Y310N additional binding to the aromatic residues at the N-terminus of Tau occurred. We conclude that $\pi-\pi$ attractive forces between the PcTS aromatic ring and the aromatic residues of Tau are responsible for the PcTS-Tau interaction. 


\section{Inhibition of Tau Filament Formation by Conformational Modulation}

a

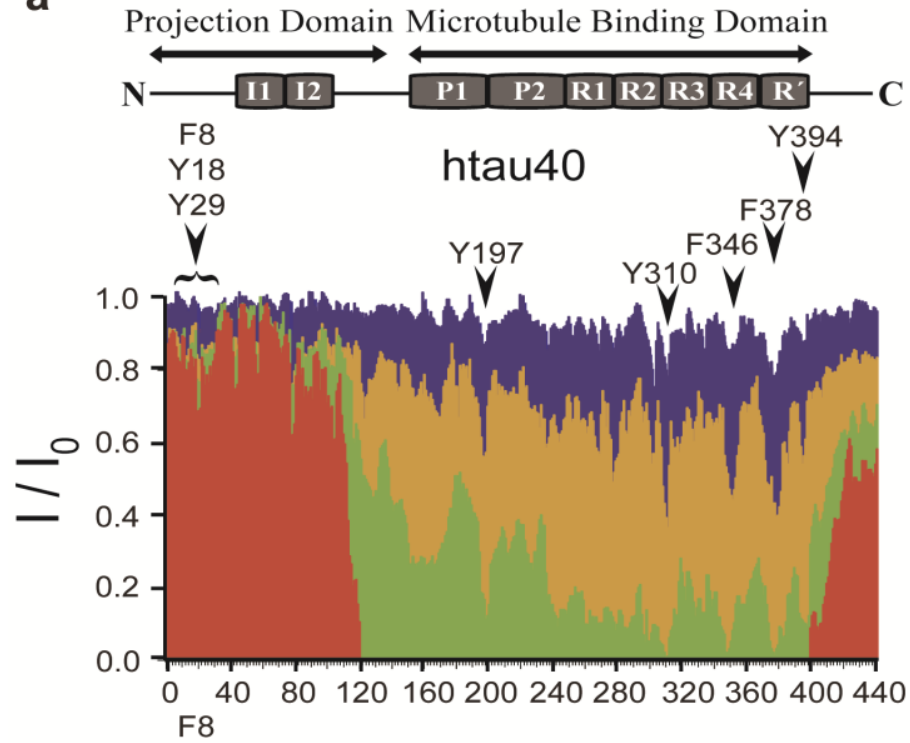

C

C Y18

Projection Domain Microtubule Binding Domain 10

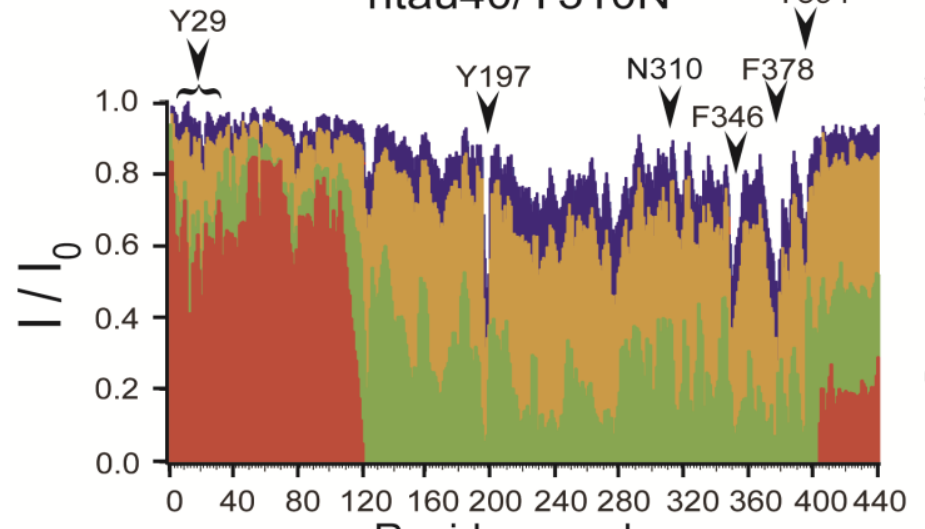

Residue number

b

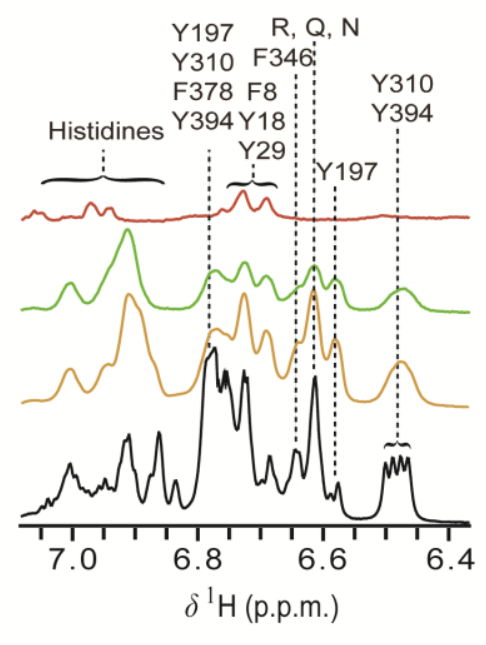

d

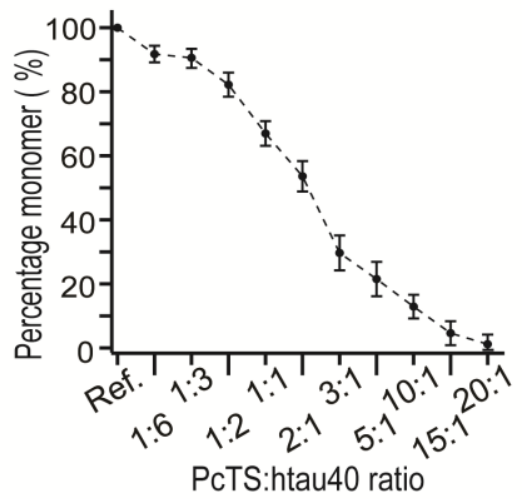

Figure 2: PcTS binds to aromatic rings of Tau.

(a) NMR signal broadening of $100 \mu \mathrm{M}$ htau 40 at increasing PcTS concentrations (PcTS:Tau 1:2 blue, 1:1 yellow, 5:1 green, 15:1 red) as a function of residue number. Aromatic residues of htau40 are marked. (b) 1D ${ }^{1} \mathrm{H}$ NMR spectra of htau 40 in the absence (black) and presence of the corresponding ratios of PcTS. (c) NMR signal broadening of the htau40/Y310N variant at increasing PcTS concentrations. (d) Decrease of the concentration of monomeric Tau at increasing PcTS concentrations. The residual monomer concentration was estimated from NMR broadening of the resonances of G120-S400 as shown in (a). 


\section{Inhibition of Tau Filament Formation by Conformational Modulation}

PcTS Stimulates Assembly of Soluble Tau Oligomers. With increasing PcTS concentrations we observed an overall decrease in NMR signal intensity for G120-S400 (Figures 2a, 2d). At 15-fold excess of PcTS, all signals in the central part of Tau were broadened beyond detection indicating that residues in this region are too immobile to be detected by liquid-state NMR. This is in line with the PcTS-driven assembly of Tau oligomers as evidenced by SDS-PAGE and electron microscopy (Figures 1d, 1e), and is supported by the disappearance of signals in ${ }^{1} \mathrm{H}-{ }^{13} \mathrm{C}$ HSQC spectra of htau24 and K18 (Figures S4, S5). Importantly, highly similar NMR broadening profiles were observed at a 10-fold lower concentration of PcTS but keeping the same PcTS:Tau molar ratio (Figure S6). As the self-stacking ability of PcTS is concentration dependent, ${ }^{32}$ the data show that the signal broadening is not due to monomeric Tau binding to large PcTS aggregates, but because of stimulation of Tau oligomer assembly by PcTS. Moreover, the NMR data reveal that not all regions in Tau are immobilized in the Tau oligomers, as residues M1-A119 and G401-L441 remained visible (Figure 2a). 


\section{Inhibition of Tau Filament Formation by Conformational Modulation}

a

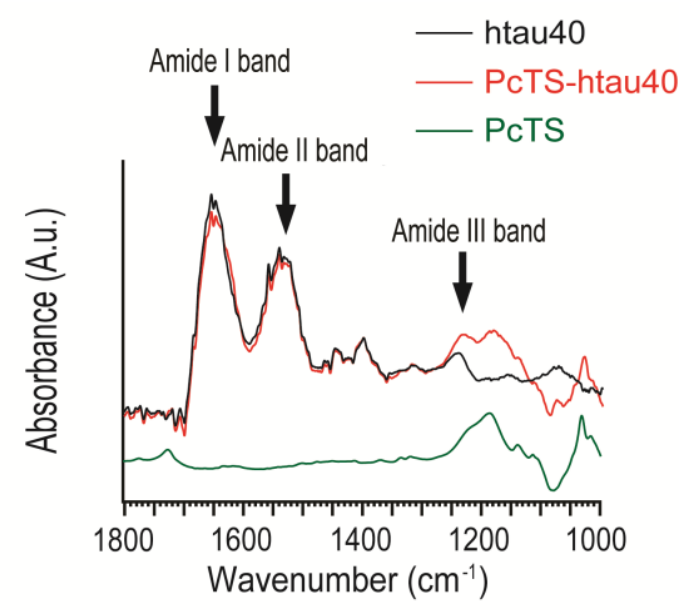

b

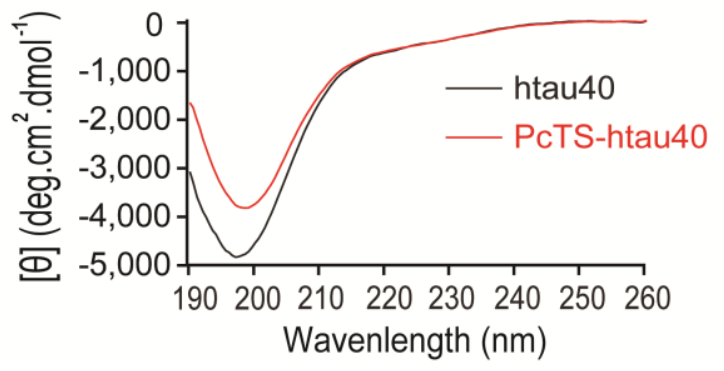

Figure 3: Secondary structure analysis of Tau oligomers.

(a) FT-IR spectra of htau40 in the absence (black) and presence of a 15-fold excess of PcTS (red). (b) Far UV-CD spectra of htau40 in the absence (black) and presence of a 15 - fold excess of PcTS (red).

Remodeling of Tau Oligomers. Biochemical analysis had revealed an increase in the concentration of SDS-resistant Tau oligomers with increasing incubation time and temperature (Figure S3). At the same time, NMR spectroscopy showed that PcTS rapidly converts Tau from the monomeric state to oligomers - the total time from preparation of the Tau-PcTS sample to completion of the $2 \mathrm{D}{ }^{1} \mathrm{H}-{ }^{15} \mathrm{~N}$ HSQC was 6 hours (Figure 2a). Thus, PcTS rapidly stimulates formation of oligomers that are mostly not resistant to SDS, but become more stable during incubation. In addition, time-dependent changes in the distribution of high molecular weight species - the $\sim 450 \mathrm{kDa}$ species becomes more pronounced - observed in SDS-PAGE point to a change in the structural properties of the PcTS-stimulated oligomers of Tau with increasing incubation time (Figure S3a). 


\section{Inhibition of Tau Filament Formation by Conformational Modulation}

Consistent with the finding, we observed time-dependent changes in the NMR spectra of Tau in the presence of 15-fold excess of PcTS. Two weeks after addition of PcTS, NMR signal intensities in the C-terminal tail of htau40 were decreased by an additional $\sim 50 \%$ (Figure S3d). The reduced signal intensities can be caused by a lower mobility of the tail, a stronger interaction with the core of the oligomer (see below) or the formation of an increased amount of oligomers, in which the C-terminal tail is rigidified and therefore not visible to NMR spectroscopy in solution.

Tau Oligomers have a Dynamic Core. Paired helical filaments (PHFs) represent fibrous assemblies of Tau with an amyloid-like core of rigid cross- $\beta$ structure. ${ }^{33}$ The formation of cross- $\beta$ structure during in vitro heparin-induced aggregation of Tau was observed by ThS in the absence of PcTS (Figure 1b). In the presence of PcTS, cross- $\beta$ structure was not formed, judging by the absence of the ThS signal (Figure 1b).

To obtain further insight into the secondary structure of PcTS oligomers, we performed CD spectroscopy and fourier transform infrared (FTIR) spectroscopy. The FTIR amide I $\left(1655 \mathrm{~cm}^{-1}\right)$ and II $\left(1541 \mathrm{~cm}^{-1}\right)$ bands are the vibrational bands that are most sensitive to protein secondary structure. However, no changes in their frequencies, and only minor changes in the other bands, were observed in the Tau oligomers when compared to monomeric Tau (Figure 3a). This is also supported by CD spectroscopy of htau40 (Figure $3 \mathrm{~b}$ ) and the repeat domain construct K18 (Figure S7). We conclude that Tau oligomers stimulated by PcTS do not contain detectable elements of $\alpha$-helix or $\beta$-structure.

To obtain information about the dynamics within Tau oligomers, we applied EPR spectroscopy to htau40 variants tagged with the paramagnetic nitroxide radical MTSL. In agreement with previous reports, ${ }^{34}$ monomeric Tau gave rise to EPR spectra with three sharp lines characteristic for a highly flexible protein backbone (Figure 4a). The EPR 


\section{Inhibition of Tau Filament Formation by Conformational Modulation}

spectra of the PcTS-induced Tau oligomer were more similar to that of monomeric Tau with three clearly distinguishable lines. Indeed for positions 15,72 and 416 no difference with respect to monomeric Tau was observed pointing to comparable mobility. However, for spin-label positions 125, 178, 239, 322, 352 and 384 EPR lines were broadened (Figures 4a, 4b), in particular for the sites 352 and 384 that are close to the aromatic residues F346, F378 and Y394 (Figures 2a, 4b). The line-broadening is in line with the burial of these sites in the Tau oligomer. At the same time, the EPR spectra of the Tau oligomers are very different from those of PHFs, which had strongly reduced amplitude and lost the outer two hyperfine lines. ${ }^{34}$ The EPR data thus demonstrate that the core of PcTS-stimulated Tau oligomers remains dynamic. 


\section{Inhibition of Tau Filament Formation by Conformational Modulation}

a

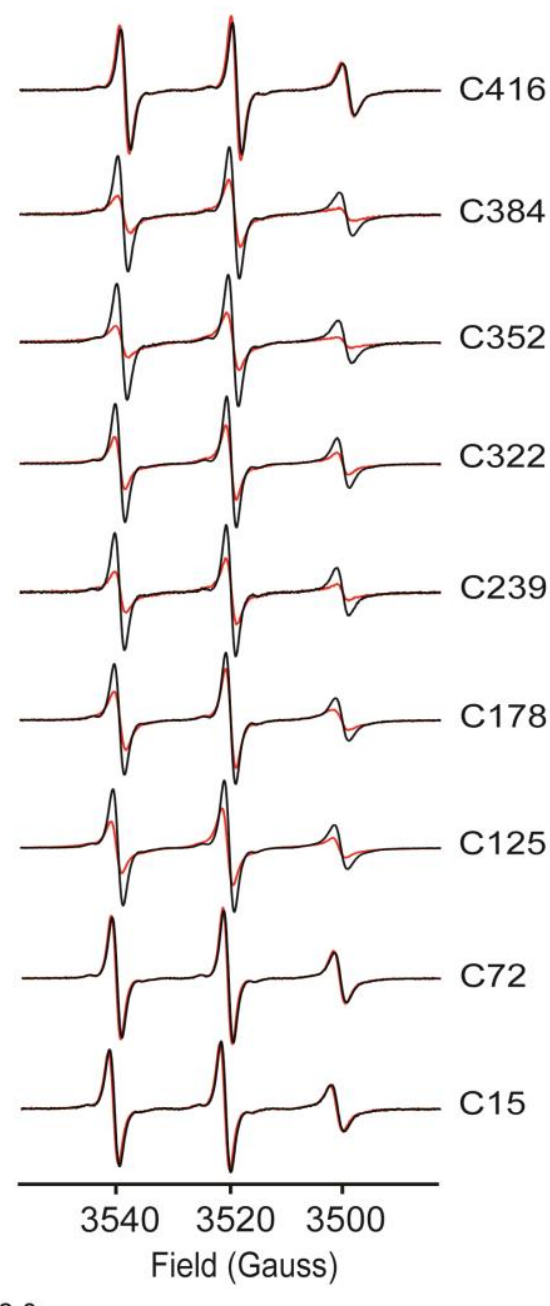

b

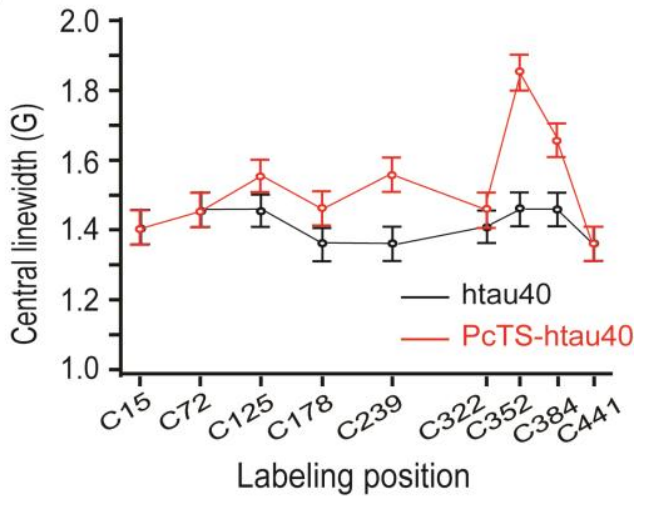

Figure 4: (a) EPR spectra of MTSL-cysteine tagged htau40 at several positions in the absence (black, monomeric Tau) and presence of PcTS (red, oligomeric Tau). (b) Comparison of the EPR linewidth of the central line in the absence (black, monomeric Tau) and presence of PcTS (red, oligomeric Tau). 


\section{Inhibition of Tau Filament Formation by Conformational Modulation}

Global Folding within Tau Oligomers. Next, we probed the long-range structure in the Tau oligomers using paramagnetic relaxation enhancement (PRE) of NMR signals ${ }^{35}$ (Figure 5). In addition, to the single cysteine mutants employed for EPR, we labeled the two native cysteines, C291 and C322, with MTSL. The paramagnetic nitroxide label results in broadening of amide resonances, which can be quantified through the NMR intensity ratios in the paramagnetic and diamagnetic states. The PRE effect scales as the inverse sixth power of the distance between the unpaired electron of the nitroxide unit and the NMR spin, providing a powerful probe of distances. Although residues in the microtubulebinding domain were too immobile to be detected, i.e. are hidden in the oligomer, the flexible tails provided sensitive probes. When spin labels were attached to the native cysteines in wild-type Tau, an approximately $10 \%$ enhancement of the NMR broadening was observed at the N-terminus when compared to monomeric Tau (Figure 5d). A more severe broadening was induced at the $\mathrm{C}$-terminus with PRE intensity ratios of $\sim 0.6$ (Figure 5d). The strong broadening at the C-terminus demonstrates a transient interaction of the flexible tails with the NMR-invisible core of the Tau oligomers. In contrast, little paramagnetic broadening was induced with a spin-label at position 239 (Figure 5c). When compared to the network of transient long-range interactions in monomeric (Figure 5 and ${ }^{8}$ ) and PHF $\mathrm{Tau}^{36}$, the interaction of the N-terminal tail with the microtubule-binding domain is weakened, while that with the C-terminus is tightened in PcTS-stimulated Tau oligomers. 


\section{Inhibition of Tau Filament Formation by Conformational Modulation}

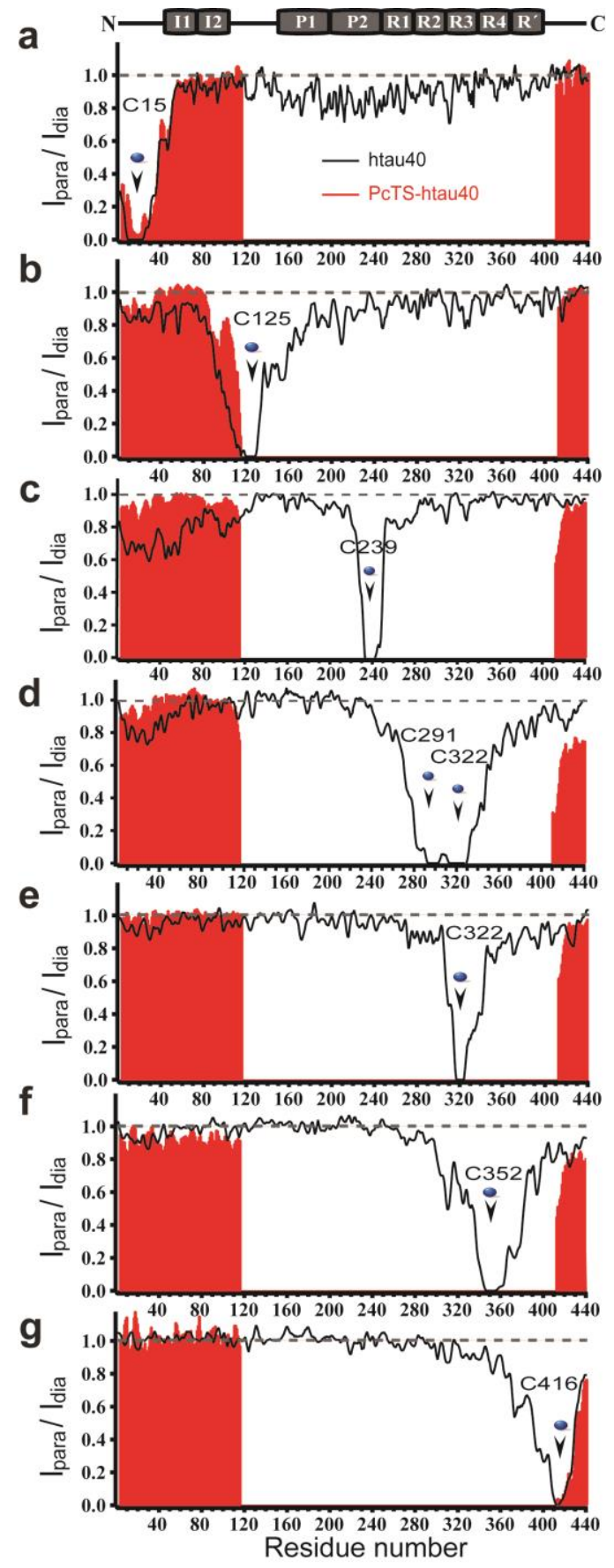

Figure 5: Changes in transient long-range interactions in Tau upon incorporation into oligomers.

Paramagnetic relaxation enhancement of backbone amide protons of MTSL spin-labeled htau40 at selected positions (a-g) in the absence (black) and presence of a 15-fold excess of PcTS (red). The plots show the intensity ratios between $2 \mathrm{D}{ }^{1} \mathrm{H}-{ }^{15} \mathrm{~N}$ HSQC spectra with the nitroxide in its paramagnetic and diamagnetic state. 


\section{Inhibition of Tau Filament Formation by Conformational Modulation}

PcTS-stimulated Tau Oligomers Have a Compact Core. At large excess of PcTS, Tau is completely converted into soluble oligomers (Figure 2d). Pulsed-field gradient NMR and dynamic light scattering showed an effective hydrodynamic radius of $\sim 68 \AA$ (Figure 6). We then performed a SAXS analysis of Tau at different protein and PcTS concentrations (Figure 7 and Figure S8). The observed SAXS profile for monomeric Tau is in line with its intrinsically disordered nature. ${ }^{37}$ However, with increasing PcTS concentrations the Kratky plot drastically changed (Figure 7b). At 15-fold excess of PcTS the Kratky plot revealed a sharp peak that is characteristic for the presence of highly compact Tau species with an average radius of gyration of $98 \pm 15 \AA$. The average radius of gyration exceeds the hydrodynamic radius by a factor of $\sim 1.4$ in line with the flexible disordered tails of the Tau oligomers detected by NMR spectroscopy (Figure 2a). In addition to the changes in the Kratky plot, the scattering intensities strongly increased at higher PcTS-Tau ratios. Noteworthy PcTS alone scattered much weaker and therefore does not contribute substantially (Figure S8a). The scattering intensities - corrected for the concentration of monomeric protein as determined by NMR spectroscopy (see materials and methods) - showed that already at an equimolar concentration oligomeric species containing approximately 5-11 Tau molecules were formed, and only slightly increased to 7-14 at 5-fold excess of PcTS (Figure 7c and Figures S7b, c). In contrast, at the higher PcTS:Tau ratios of 10:1 and 15:1 the oligomeric species shifted to an average of $\sim 24$ Tau molecules. The maximum diameter, Dmax, of the two oligomeric species was calculated as $393 \pm 10 \AA$ and $348 \pm 27 \AA$, respectively (Figure 7d). The slightly smaller Dmax value at higher PcTS is supported by the sharpening of the peak in the Kratky plot (Figure 7b). Consistent with these observations, SDS-page analysis revealed two predominant PcTSinduced Tau species with approximate molecular weights of 300 and $450 \mathrm{kDa}$ (Figure 1e). 


\section{Inhibition of Tau Filament Formation by Conformational Modulation}

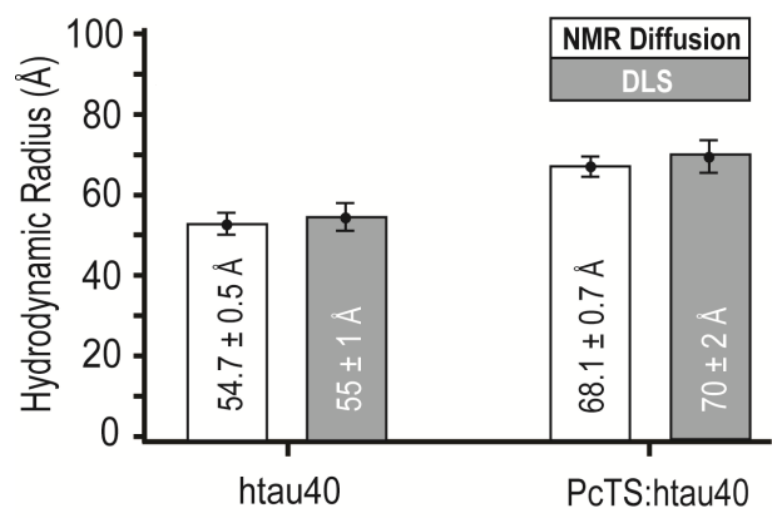

Figure 6: Hydrodynamic radius of monomeric and PcTS-stimulated oligomeric htau 40 determined by pulsed-field gradient NMR and dynamic light scattering.

a

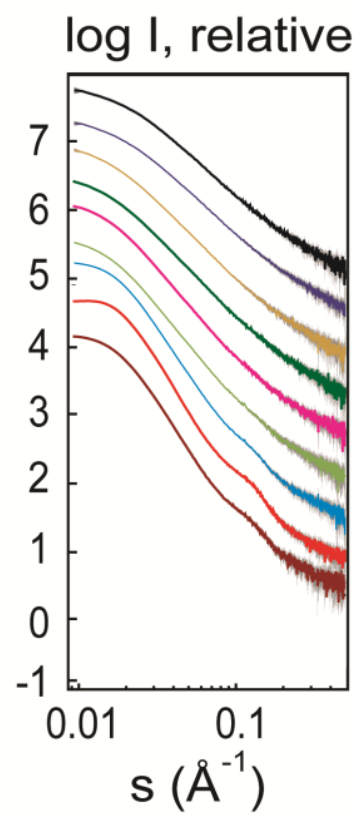

b

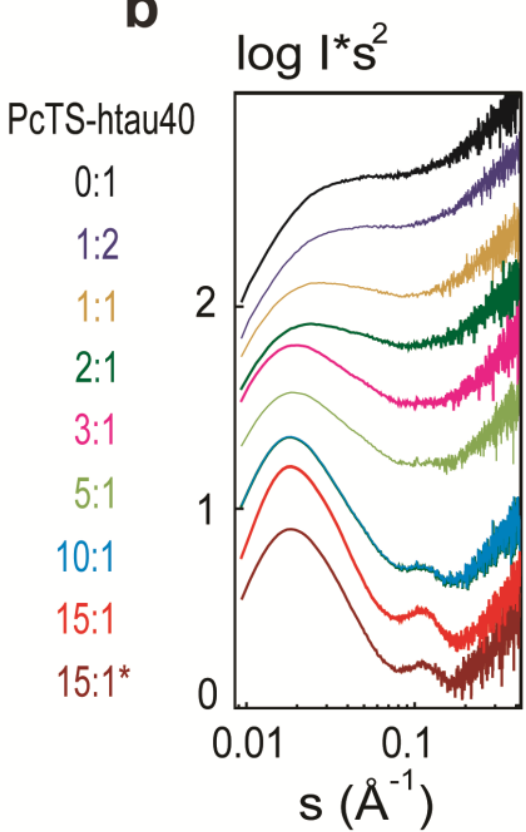

C

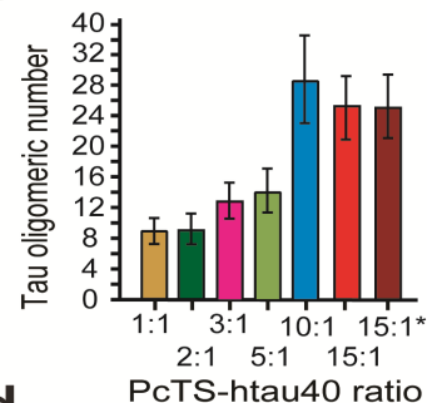

d

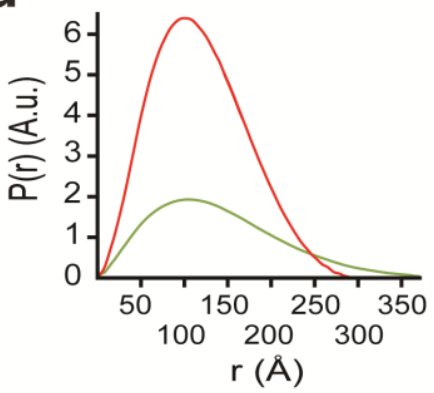

Figure 7: Low-resolution SAXS models of Tau oligomers.

(a) Experimental SAXS spectra and (b) Kratky plots of $10 \mathrm{mg} / \mathrm{ml}$ hau 40 (black) alone and in the presence of increasing concentrations of PcTS (color coded panel). Note that the PcTS signal does not contribute significantly to the total scattering intensity (Figure S8). (c) Estimation of the number of Tau molecules in PcTS-stimulated oligomers at different PcTS:htau40 ratios (with htau40 at $2.5 \mathrm{mg} / \mathrm{ml}$ ). The dark red bar shows the data for the 15:1 sample after filtering. Error bars were estimated from the standard error of $\mathrm{I}_{0}$ variations between different concentrations of htau 40 but at the same ratio of PcTS and accounting for errors in the NMR-based estimation of the concentration of monomeric htau40 (see Supporting Information). (d) The $P(r)$ of Tau oligomers at 5 -fold (green) and 15-fold (red) excess of PcTS. 


\section{Inhibition of Tau Filament Formation by Conformational Modulation}

Tau Oligomers are Stabilized Non-cooperatively. To obtain a detailed description of the conformational stability of different regions in the Tau oligomers, we performed equilibrium denaturation experiments. ${ }^{38}$ A comparison of NMR spectra obtained in $8 \mathrm{M}$ urea and $3 \mathrm{M}$ GuSCN revealed a distinct sensitivity of the Tau oligomers to the two denaturants (Figures 8a-c and Figure S9). All observable residues were classified into one of four groups depending on their urea denaturation profile (Figures $8 \mathrm{~d}-\mathrm{e}$ ). The denaturation profiles differed between the groups, but within a group residues displayed cooperative behaviour (Figure 8e). Surprisingly, residues G120-T205 belonging to group III, which are broadened beyond detection in the Tau oligomers, were very sensitive to low concentrations of urea. In contrast, residues in the second proline-rich region and the repeats were not detected even at $8 \mathrm{M}$ urea demonstrating that they form the core of PcTSstimulated oligomers of Tau. The resistance to urea denaturation is in line with $\pi-\pi$ stacking as a key factor for oligomer stability.

Residues belonging to group I and II from the urea denaturation roughly coincide with residues having a common denaturation profile in GuSCN (GuSCN group I) (Figures $8 \mathrm{f}-\mathrm{g})$. Strikingly, in the repeat region signals were detected already at $0.1 \mathrm{mM} \mathrm{GuSCN}$, demonstrating a very low stability of the Tau oligomers in ionic denaturants. In addition, signal intensities in $3 \mathrm{M} \mathrm{GuSCN}$ were highly similar to those observed in monomeric Tau (Figure S10). The different denaturation profiles for individual residues indicate that the PcTS-stimulated Tau oligomers are noncooperatively stabilized.

PcTS Inhibits Tau Aggregation in Cells. To investigate the impact of PcTSinduced oligomer formation on Tau aggregation in cells, we used the Neuroblastoma N2a cell model. ${ }^{39}$ After 4 days of incubation at $37{ }^{\circ} \mathrm{C}$, the fluorescence signal intensity of the ThS-positive aggregates was measured. The amount of ThS-positive cells in the untreated 


\section{Inhibition of Tau Filament Formation by Conformational Modulation}

positive control was set to $100 \%$. From this cell-based assay, we observe that PcTS leads to a $97 \%$ reduction in the fluorescence of $\mathrm{ThS}$-positive cells relative to the untreated positive control (Figure 9), demonstrating that PcTS is a highly effective inhibitor of Tauinduced aggregation in cells.

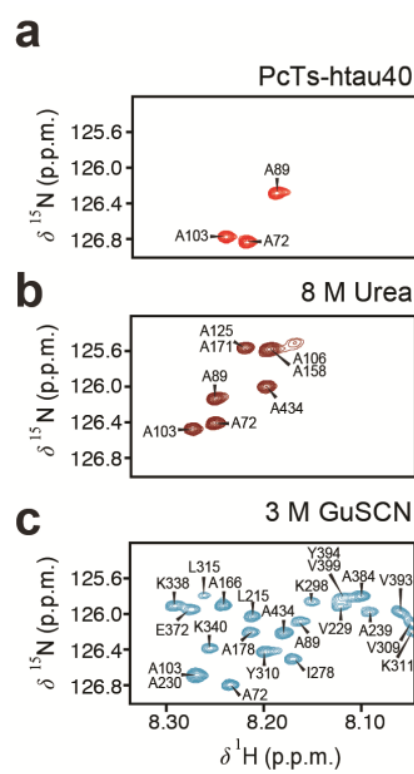

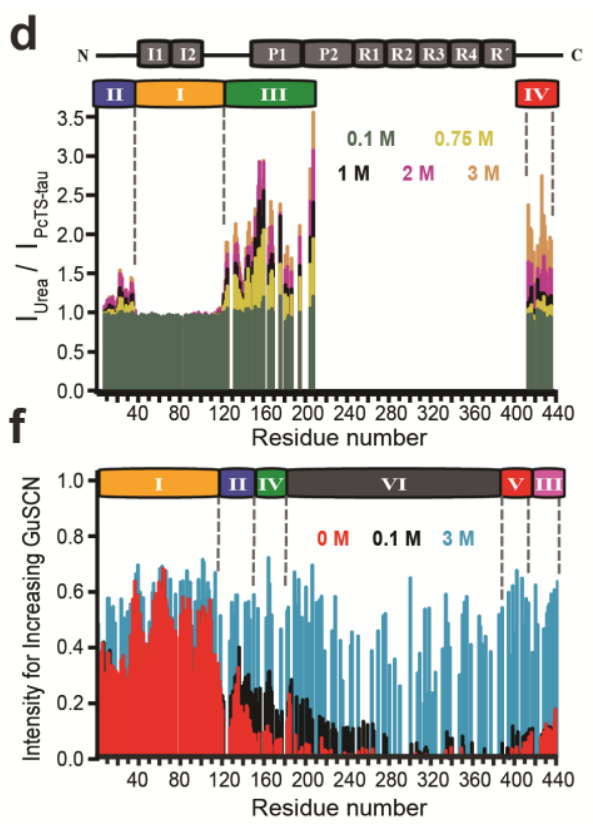

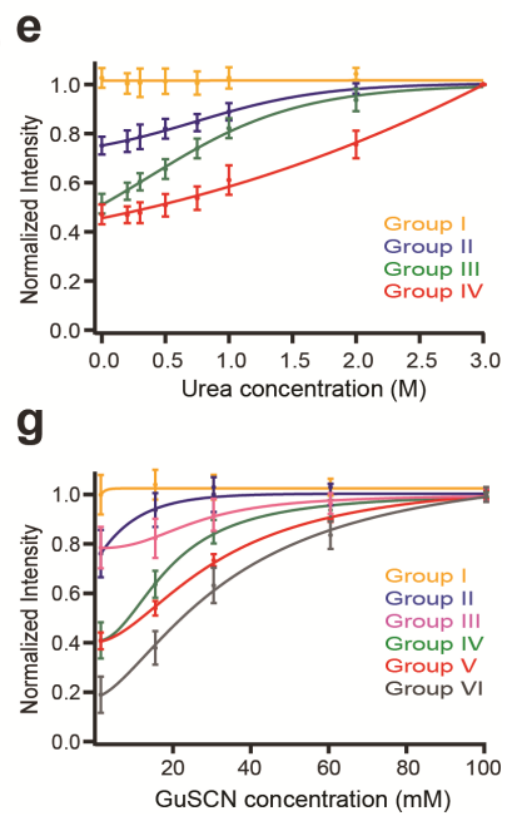

Figure 8: Stability of Tau oligomers.

(a) Selected region of $2 \mathrm{D}{ }^{1} \mathrm{H}-{ }^{15} \mathrm{~N}$ HSQC spectra of htau40 in the presence of a 15 -fold excess of PcTS, and after addition of $8 \mathrm{M}$ urea (b) and $3 \mathrm{M}$ GuSCN (C). Resonances of residues that were rigidified in the Tau oligomers were partially (b) or fully (c) recovered. (d) Ratios of NMR signal intensities in $2 \mathrm{D}{ }^{1} \mathrm{H}-{ }^{15} \mathrm{~N}$ HSQC spectra of htau40 with 15-fold excess of PcTS at increasing urea concentrations $\left(\mathrm{I}_{\text {urea }}\right)$ when compared to values in the absence of urea ( $\mathrm{I}_{\mathrm{PcTS} \text {-Tau }}$ ). Increasing signal intensities indicate a residue-specific destabilization of the Tau oligomers. (e) Urea denaturation profiles of PcTS-stimulated Tau oligomers. All observable residues were classified into one of four groups based on their initial intensities, their sensitivity to urea concentration and their intensities in $3 \mathrm{M}$ urea. The denaturation profiles differed between the groups, but within a group residues displayed cooperative behaviour. (f) Residue-specific analysis of NMR signal intensities of PcTS:htau40 (15:1) at increasing concentrations of GuSCN. Only non-overlapping peaks were included. Groups of residues (I-VI) that show a common profile of denaturation are marked above and their denaturation profiles are shown in $(\mathrm{g})$. 


\section{Inhibition of Tau Filament Formation by Conformational Modulation}

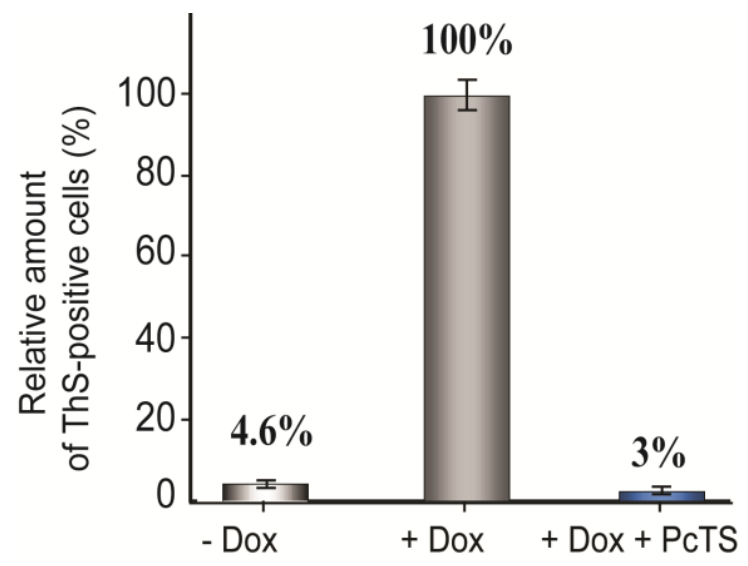

Figure 9: Percentage of ThS-positive cells determined by a FACS analysis of inducible N2a cells expressing the four-repeat domain pro-aggregation $\mathrm{K} 18 \Delta \mathrm{K} 280$ construct.

Cell suspensions containing either ThS alone (negative control) or ThS and doxycyclin without (positive control) or with $50 \mu \mathrm{M}$ PcTS (treated cell culture) were incubated for 4 days at $37{ }^{\circ} \mathrm{C}$. The fluorescence signal intensity of ThS-positive aggregates was measured and the amount of cells in the untreated positive control was set to $100 \%$.

\section{DISCUSSION}

Our study demonstrates that the organic compound Phthalocyanine tetrasulfonate binds to specific aromatic residues of Tau and inhibits Tau filament formation by targeting Tau into soluble oligomers. Using a combination of NMR spectroscopy, EPR spectroscopy and SAXS two predominant oligomeric species were revealed comprising $\sim 7-14$ and 24 Tau molecules, respectively. The core of the soluble Tau oligomers is highly compact with a maximum diameter of 30-40 $\mathrm{nm}$ (Figure 7), but is non-cooperatively stabilized (Figure 8). Biochemical experiments and analyses in Tau animal models suggested that Tau oligomers and not PHFs might represent the acutely toxic species. ${ }^{9,40}$ Tau oligomers prepared in vitro, which impair memory and cause synaptic dysfunction in mice, ${ }^{41}$ were largely SDS - stable apparent trimers, displayed a spherical morphology and contained $\beta$ - 


\section{Inhibition of Tau Filament Formation by Conformational Modulation}

sheet structure. ${ }^{42}$ In contrast, Tau oligomers stimulated by PcTS are only partially SDSstable, are not spherical and do not contain $\beta$-structure (Figures 1-7), pointing to distinct structural properties of toxic Tau oligomers.

Despite the highly compact core of PcTS-stimulated Tau oligomers significant heterogeneity is likely to remain both with respect to the species being present and the number of Tau molecules in the oligomeric species. EPR spectroscopy demonstrated that even the compact core of Tau oligomers remains highly dynamic (Figure 4). The dynamic nature of the oligomer is in clear contrast to the pronounced rigidity of the $\beta$-sheet rich core of Tau amyloid fibrils ${ }^{34}$. In addition, the EPR line-broadening observed for the spinlabels at position 352 and 384 (Figure 4) - in comparison to spin-label positions in the Cterminal domain - suggest that this region is more rigid or compact in Tau oligomers. This region also contains the three aromatic residues F346, F378 and Y394 that comprise the primary binding sites for PcTS (Figure 2a). In contrast, the $\beta$-sheet rich core of PHFs is located more to the $\mathrm{N}$-terminal part of the repeat region extending at most to residue $\sim 355 .^{43-45}$ Thus, the location of the compact core of PcTS-stimulated Tau oligomers and Tau filaments is partially distinct in line with the finding that PcTS-stimulated Tau oligomers cannot convert into Tau filaments. 


\section{Inhibition of Tau Filament Formation by Conformational Modulation}

\section{CONCLUSION}

PcTS belongs to the family of cyclic tetrapyrroles, is planar with a hydrophobic central aromatic macrocyclic structure, and carries four negatively charged sulfonate groups at its peripheral outer benzo substitution positions. Previously, we showed that PcTS binds to the aromatic residues of $\alpha$-synuclein in the monomeric protein. ${ }^{22,46,47}$ Here we demonstrate that PcTS inhibits formation of Tau filaments in vitro and in cells (Figures 1,9) by selectively interacting with residues Y197, Y310, F346, F378 and Y394 in the central domain of Tau and converting the Tau protein into soluble oligomers. The specific interaction with aromatic residues in the central domain of Tau - and not with aromatic residues at the N-terminus - is distinct from a non-selective sequestration of protein molecules due to self-association of anti-amyloid agents. ${ }^{48}$ It is also distinct from the nonselective mechanism that involves the covalent linkage via lysine residues into highmolecular weight oligomers as suggested for the polyphenol oleocanthal. ${ }^{19,49}$

Site-directed mutagenesis indicated that the different PcTS-binding sites in Tau are independent (Figure 2c). In addition, the observation that soluble Tau oligomers are formed in the absence of a single residue, Y310, supports a key role of PcTS-stimulated oligomer formation for the inhibition of Tau aggregation. While Y310 is buried in the fibrillar core of PHFs, ${ }^{36,43}$ Y197 and Y394 remain accessible for interaction with PcTS providing a mechanism for the previously demonstrated dissolvation of Tau filaments by PcTS. ${ }^{16}$

In summary, we revealed detailed insights into the mechanism of Tau aggregation inhibition and the structure and dynamics of soluble Tau oligomers. Our study demonstrates that the structure of off-pathway oligomers of Tau is distinct from the structure of toxic Tau oligomers. 


\begin{abstract}
ASSOCIATED CONTENT
Supporting Information. Overview over Tau isoforms; changes in chemical shifts of Tau induced by PcTS; time-dependent changes in NMR intensity profiles; interaction of PcTS with the Tau fragment K18 and Tau isoform htau24; influence of PcTS concentration on NMR broadening. This material is available free of charge via the Internet at http://pubs.acs.org.
\end{abstract}

\title{
AUTHOR INFORMATION
}

\section{Corresponding Authors}

Markus.Zweckstetter@dzne.de

Eckhard.Mandelkow@dzne.de

\section{ACKNOWLEDGMENTS}

We thank Marina Bennati and Eva-Maria Mandelkow for stimulating discussion and Ilka Lindner for sample preparation. This work was funded through the Cluster of Excellence and DFG Research Center "Nanoscale Microscopy and Molecular Physiology of the Brain", the MPG consortium Toxic Protein Conformation, the Metlife foundation, the Wellcome Trust and the DFG (ZW 71/3-2 to M.Z.). 


\section{Inhibition of Tau Filament Formation by Conformational Modulation}

\section{ABBREVIATIONS}

AD, Alzheimer's Disease; htau40; 441-residue Tau protein; PHFs, paired helical filaments; PcTS, Phthalocyanine tetrasulfonate; NMR, nuclear magnetic resonance; HSQC, heteronuclear single quantum coherence; EPR, electron paramagnetic resonance; SAXS, small-angle X-ray scattering; ThS, thioflavinS; SDS-Page, sodium dodecyl sulfate polyacrylamide gel electrophoresis SDS-PAGE; FTIR, fourier transform infrared.

\section{REFERENCES}

(1) Ross, C. A.; Poirier, M. A. Nat Med 2004, 10 Suppl, S10.

(2) Forman, M. S.; Lee, V. M.; Trojanowski, J. Q. J Chem Neuroanat 2000, 20, 225.

(3) Hardy, J.; Selkoe, D. J. Science 2002, 297, 353.

(4) Goedert, M.; Spillantini, M. G. Science 2006, 314, 777.

(5) Cleveland, D. W.; Hwo, S. Y.; Kirschner, M. W. J Mol Biol 1977, 116, 227.

(6) Schweers, O.; Schonbrunn-Hanebeck, E.; Marx, A.; Mandelkow, E. J. Biol. Chem. 1994, 269, 24290.

(7) Drubin, D.; Kirschner, M. J. Cell Biol. 1986, 103, 2739.

(8) Mukrasch, M. D.; Bibow, S.; Korukottu, J.; Jeganathan, S.; Biernat, J.; Griesinger, C.; Mandelkow, E.; Zweckstetter, M. PLoS Biol 2009, 7, e34.

(9) Lasagna-Reeves, C. A.; Castillo-Carranza, D. L.; Jackson, G. R.; Kayed, R. Curr Alzheimer Res 2011, 8, 659.

(10) Pul, R.; Dodel, R.; Stangel, M. Expert Opin Biol Ther 2011, 11, 343.

(11) Noble, W.; Planel, E.; Zehr, C.; Olm, V.; Meyerson, J.; Suleman, F.; Gaynor, K.; Wang, L.; LaFrancois, J.; Feinstein, B.; Burns, M.; Krishnamurthy, P.; Wen, Y.; Bhat, R.; Lewis, J.; Dickson, D.; Duff, K. Proc Natl Acad Sci U S A 2005, 102, 6990. 


\section{Inhibition of Tau Filament Formation by Conformational Modulation}

(12) Zhang, B.; Maiti, A.; Shively, S.; Lakhani, F.; McDonald-Jones, G.; Bruce, J.; Lee, E. B.; Xie, S. X.; Joyce, S.; Li, C.; Toleikis, P. M.; Lee, V. M.; Trojanowski, J. Q. Proc Natl Acad Sci U S A 2005, 102, 227.

(13) Wischik, C. M.; Edwards, P. C.; Lai, R. Y.; Roth, M.; Harrington, C. R. Proc Natl Acad Sci U S A 1996, 93, 11213.

(14) Pickhardt, M.; von Bergen, M.; Gazova, Z.; Hascher, A.; Biernat, J.; Mandelkow, E. M.; Mandelkow, E. Curr Alzheimer Res 2005, 2, 219.

(15) Crowe, A.; Ballatore, C.; Hyde, E.; Trojanowski, J. Q.; Lee, V. M. Biochem Biophys Res Commun 2007, 358, 1.

(16) Taniguchi, S.; Suzuki, N.; Masuda, M.; Hisanaga, S.; Iwatsubo, T.; Goedert, M.; Hasegawa, M. J. Biol. Chem. 2005, 280, 7614.

(17) Bulic, B.; Pickhardt, M.; Schmidt, B.; Mandelkow, E. M.; Waldmann, H.; Mandelkow, E. Angew Chem Int Ed Engl 2009, 48, 1740.

(18) Chirita, C.; Necula, M.; Kuret, J. Biochemistry 2004, 43, 2879.

(19) Li, W.; Sperry, J. B.; Crowe, A.; Trojanowski, J. Q.; Smith, A. B., 3rd; Lee, V. M. J Neurochem 2009, 110, 1339.

(20) Ehrnhoefer, D. E.; Bieschke, J.; Boeddrich, A.; Herbst, M.; Masino, L.; Lurz, R.; Engemann, S.; Pastore, A.; Wanker, E. E. Nat Struct Mol Biol 2008, 15, 558.

(21) Woods, L. A.; Platt, G. W.; Hellewell, A. L.; Hewitt, E. W.; Homans, S. W.; Ashcroft, A. E.; Radford, S. E. Nat Chem Biol 2011, 7, 730.

(22) Lamberto, G. R.; Binolfi, A.; Orcellet, M. L.; Bertoncini, C. W.; Zweckstetter, M.; Griesinger, C.; Fernandez, C. O. Proc Natl Acad Sci U S A 2009, 106, 21057.

(23) Caughey, W. S.; Raymond, L. D.; Horiuchi, M.; Caughey, B. Proc Natl Acad Sci U S A 1998, 95, 12117.

(24) Priola, S. A.; Raines, A.; Caughey, W. S. Science 2000, 287, 1503.

(25) Park, J. W.; Ahn, J. S.; Lee, J. H.; Bhak, G.; Jung, S.; Paik, S. R. Chembiochem 2008, 9, 2602. 


\section{Inhibition of Tau Filament Formation by Conformational Modulation}

(26) Mukrasch, M. D.; Biernat, J.; von Bergen, M.; Griesinger, C.; Mandelkow, E.; Zweckstetter, M. J Biol Chem 2005, 280, 24978.

(27) Delaglio, F.; Grzesiek, S.; Vuister, G. W.; Zhu, G.; Pfeifer, J.; Bax, A. J. Biomol. NMR. 1995, 6, 277.

(28) Zheng, G.; Stait-Gardner, T.; Anil Kumar, P. G.; Torres, A. M.; Price, W. S. J Magn Reson 2008, 191, 159.

(29) Jones, J. A.; Wilkins, D. K.; Smith, L. J.; Dobson, C. M. J. Biomol. NMR. 1997, 10, 199.

(30) Konarev, P. V.; Petoukhov, M. V.; Volkov, V. V.; Svergun, D. I. J. Appl. Cryst. 2006, $39,277$.

(31) Goedert, M.; Jakes, R.; Spillantini, M. G.; Hasegawa, M.; Smith, M. J.; Crowther, R.

A. Nature 1996, 383, 550.

(32) Boyle, R. W.; Dolphin, D. Photochem Photobiol 1996, 64, 469.

(33) Berriman, J.; Serpell, L. C.; Oberg, K. A.; Fink, A. L.; Goedert, M.; Crowther, R. A. Proc Natl Acad Sci US A 2003, 100, 9034.

(34) Margittai, M.; Langen, R. Proc Natl Acad Sci U S A 2004, 101, 10278.

(35) Gillespie, J. R.; Shortle, D. J Mol Biol 1997, 268, 170.

(36) Bibow, S.; Mukrasch, M. D.; Chinnathambi, S.; Biernat, J.; Griesinger, C.; Mandelkow, E.; Zweckstetter, M. Angew Chem Int Ed Engl 2011, 50, 11520.

(37) Mylonas, E.; Hascher, A.; Bernado, P.; Blackledge, M.; Mandelkow, E.; Svergun, D. I. Biochemistry 2008, 47, 10345.

(38) Schulman, B. A.; Kim, P. S.; Dobson, C. M.; Redfield, C. Nat Struct Biol 1997, 4, 630.

(39) Pickhardt, M.; Biernat, J.; Khlistunova, I.; Wang, Y. P.; Gazova, Z.; Mandelkow, E. M.; Mandelkow, E. Curr Alzheimer Res 2007, 4, 397.

(40) Brunden, K. R.; Trojanowski, J. Q.; Lee, V. M. J Alzheimers Dis 2008, 14, 393.

(41) Lasagna-Reeves, C. A.; Castillo-Carranza, D. L.; Sengupta, U.; Clos, A. L.; Jackson, G. R.; Kayed, R. Mol Neurodegener 2011, 6, 39. 


\section{Inhibition of Tau Filament Formation by Conformational Modulation}

(42) Lasagna-Reeves, C. A.; Castillo-Carranza, D. L.; Guerrero-Muoz, M. J.; Jackson, G. R.; Kayed, R. Biochemistry 2010, 49, 10039.

(43) Andronesi, O. C.; von Bergen, M.; Biernat, J.; Seidel, K.; Griesinger, C.; Mandelkow, E.; Baldus, M. J Am Chem Soc 2008, 130, 5922.

(44) Daebel, V.; Chinnathambi, S.; Biernat, J.; Schwalbe, M.; Habenstein, B.; Loquet, A.; Akoury, E.; Tepper, K.; Muller, H.; Baldus, M.; Griesinger, C.; Zweckstetter, M.; Mandelkow, E.; Vijayan, V.; Lange, A. J Am Chem Soc 2012, 134, 13982.

(45) von Bergen, M.; Barghorn, S.; Muller, S. A.; Pickhardt, M.; Biernat, J.; Mandelkow, E. M.; Davies, P.; Aebi, U.; Mandelkow, E. Biochemistry 2006, 45, 6446.

(46) Rao, J. N.; Dua, V.; Ulmer, T. S. Biochemistry 2008, 47, 4651.

(47) Lamberto, G. R.; Torres-Monserrat, V.; Bertoncini, C. W.; Salvatella, X.; Zweckstetter, M.; Griesinger, C.; Fernandez, C. O. J. Biol. Chem. 2011, 286, 32036.

(48) McGovern, S. L.; Caselli, E.; Grigorieff, N.; Shoichet, B. K. J Med Chem 2002, 45, 1712.

(49) Monti, M. C.; Margarucci, L.; Tosco, A.; Riccio, R.; Casapullo, A. Food Funct 2011, 2,423 . 


\section{SUPPORTING INFORMATION}

\section{SUPPORTING FIGURES}

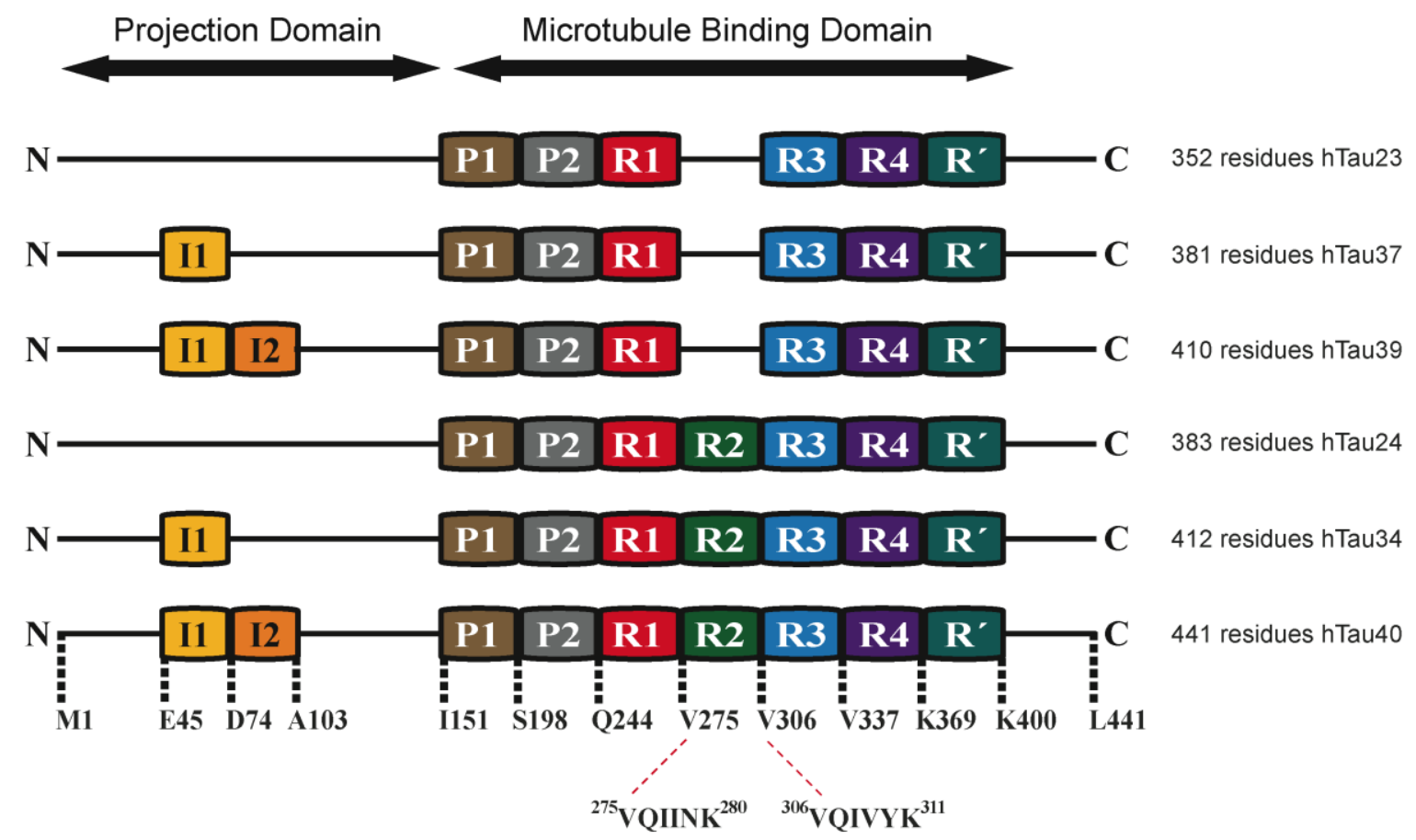

Figure S1: Isoforms of human Tau protein.

Six isoforms of Tau protein exist in the human central nervous system and are generated by alternative splicing of the MAPT gene. The number of the insert regions in the projection domain $(0,1$ or 2$)$ and the presence or absence of the second repeat region in the microtubule-binding domain distinguish them. $\mathrm{N}$ or $\mathrm{C}=\mathrm{N}$ or $\mathrm{C}-$ terminus, $\mathrm{I}=$ insert, $\mathrm{P}=$ proline-rich region, and $\mathrm{R}=$ repeat region. 


\section{Inhibition of Tau Filament Formation by Conformational Modulation}

a

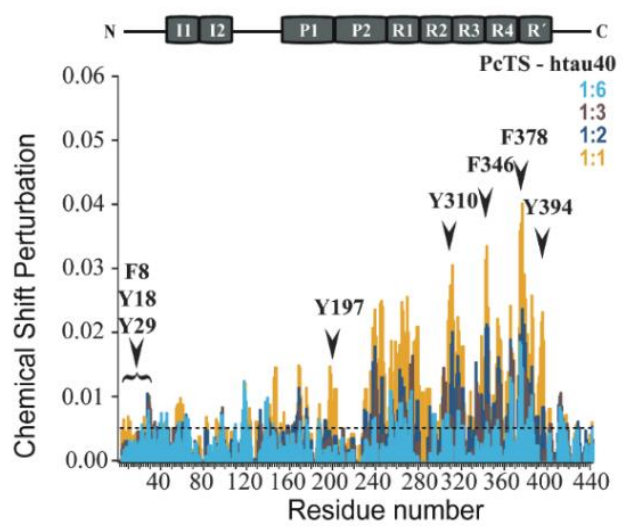

b

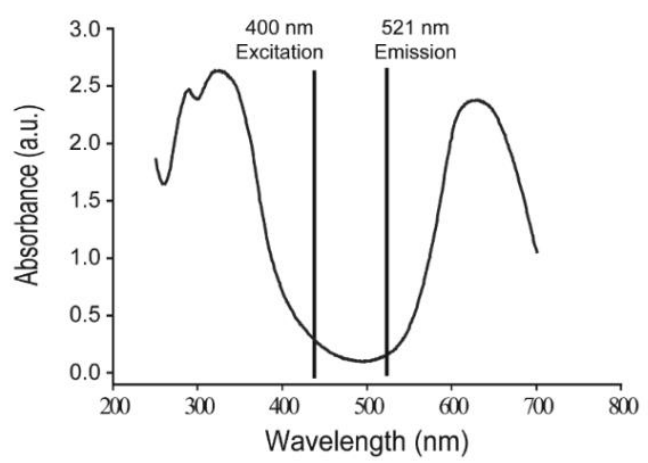

C
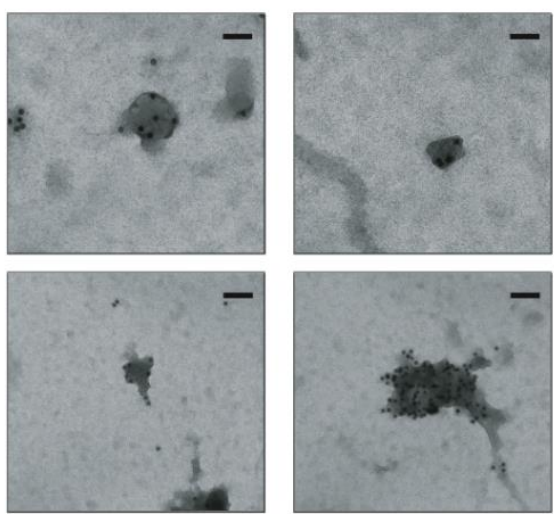

Figure S2: (a) Chemical shift deviations $\left(\Delta \delta_{1 \mathrm{H} / 15 \mathrm{Ntit}}-\Delta \delta_{1 \mathrm{H} / 15 \mathrm{Nref}}\right)$ observed in $2 \mathrm{D}{ }^{1} \mathrm{H}-{ }^{15} \mathrm{~N}$ HSQC NMR spectra of htau40wt at increasing PcTS concentrations. Averaged, normalized chemical shift deviations were calculated according to $\Delta \delta_{\mathrm{AV}}=\left[0.5\left(\delta_{\mathrm{Htit}}-\delta_{\mathrm{Hwt}}\right)^{2}+0.02\left(\delta_{\mathrm{Ntit}}-\delta_{\mathrm{Nwt}}\right)^{2}\right]^{1 / 2}$. Dotted black lines correspond to thresholds of chemical shift perturbations of 0.005 ppm for PcTS. (b) Absorption profile of PcTS demonstrating that the absorption properties of PcTS do not interfere with ThS fluorescence in the working range (excitation and emission wavelengths of $440 \mathrm{~nm}$ and $521 \mathrm{~nm}$, respectively). (c) Electron micrographs of PcTS-htau40 oligomers. Scale bars, $40 \mathrm{~nm}$ (upper panels) and $70 \mathrm{~nm}$ (lower panels). The black dots represent the gold-labelled antibody K9JA-gold $10 \mathrm{~nm}$ used for immunostaining. 


\section{Inhibition of Tau Filament Formation by Conformational Modulation}

a

Gel a: $1 \mathrm{~h}$ incubation

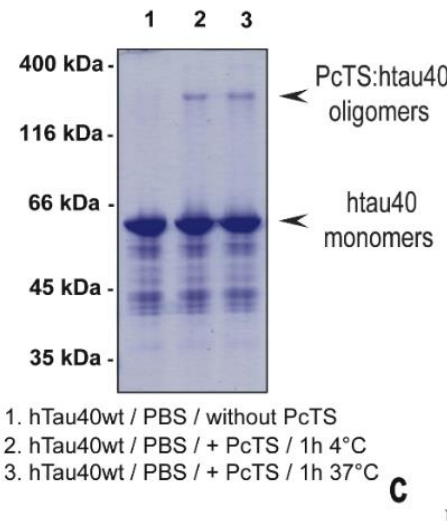

b

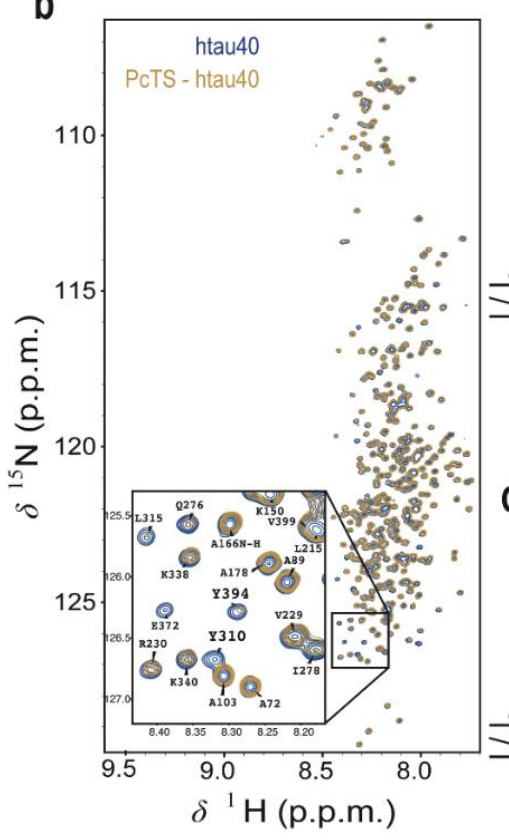

Gel b: $24 \mathrm{~h}$ incubation

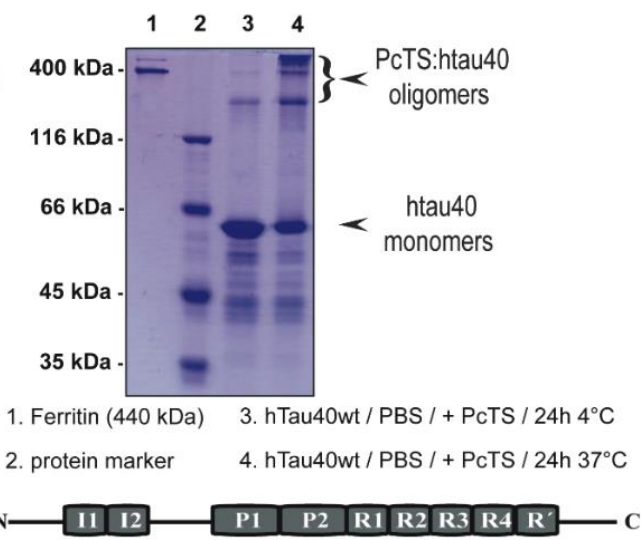

PcTS - htau40 1to 1

6 hours

3 days

7 days

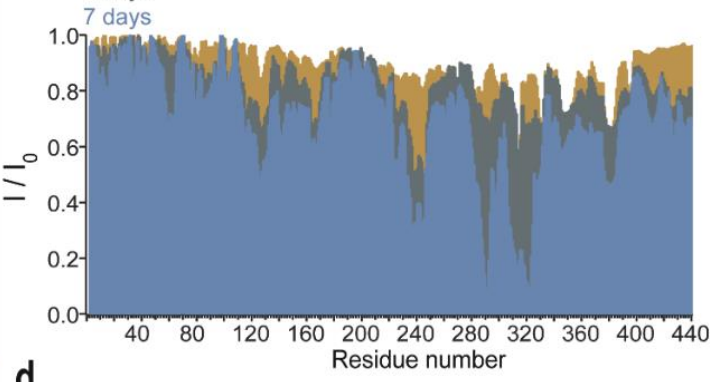

d PcTS - hTau40 15to1

6 hours

2 weeks

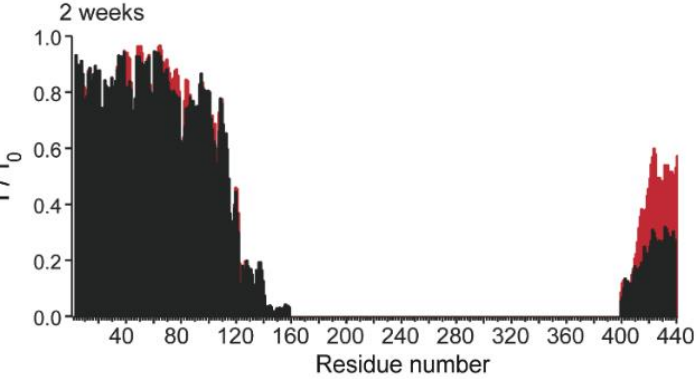

Figure S3: Time-dependent remodeling of the PcTS-stimulated Tau oligomers.

(a) PcTS-induced oligomers visualized by SDS-PAGE. $50 \mu \mathrm{M}$ monomeric htau 40 samples containing $1 \mathrm{mM}$ DTT were prepared in PBS buffer (pH 7.4) and heated for 20 minutes at $95{ }^{\circ} \mathrm{C}$. After incubation with 750 $\mu \mathrm{M}$ PcTS for 1 or 24 hours at 4 or $37^{\circ} \mathrm{C}$, all samples were run on SDS-PAGE gels together with reference (Ferritin $440 \mathrm{kDa}$ and protein marker) and control samples (monomeric htau40 in PBS buffers). The samples incubated for 1 hour at 4 and $37{ }^{\circ} \mathrm{C}$ show a protein band of higher molecular weight (gel a line 3 and 4). Further 23 hours of incubation, resulted in an intensity increase and the appearance of additional protein bands with a considerable decrease of the monomeric htau40 signal (comparison of gel a line 4 with gel b line 4). (b) Superposition of $2 \mathrm{D}{ }^{1} \mathrm{H}-{ }^{15} \mathrm{~N}$ HSQC spectra of $100 \mu \mathrm{M}$ htau 40 in the absence (blue) and presence (yellow) of an equimolar ratio of PcTS. (c,d) Time-dependence of NMR signal intensity ratios of htau 40 for (c) 1:1 PcTS-htau40 and (d) 15:1 PcTS-htau40. 


\section{Inhibition of Tau Filament Formation by Conformational Modulation}

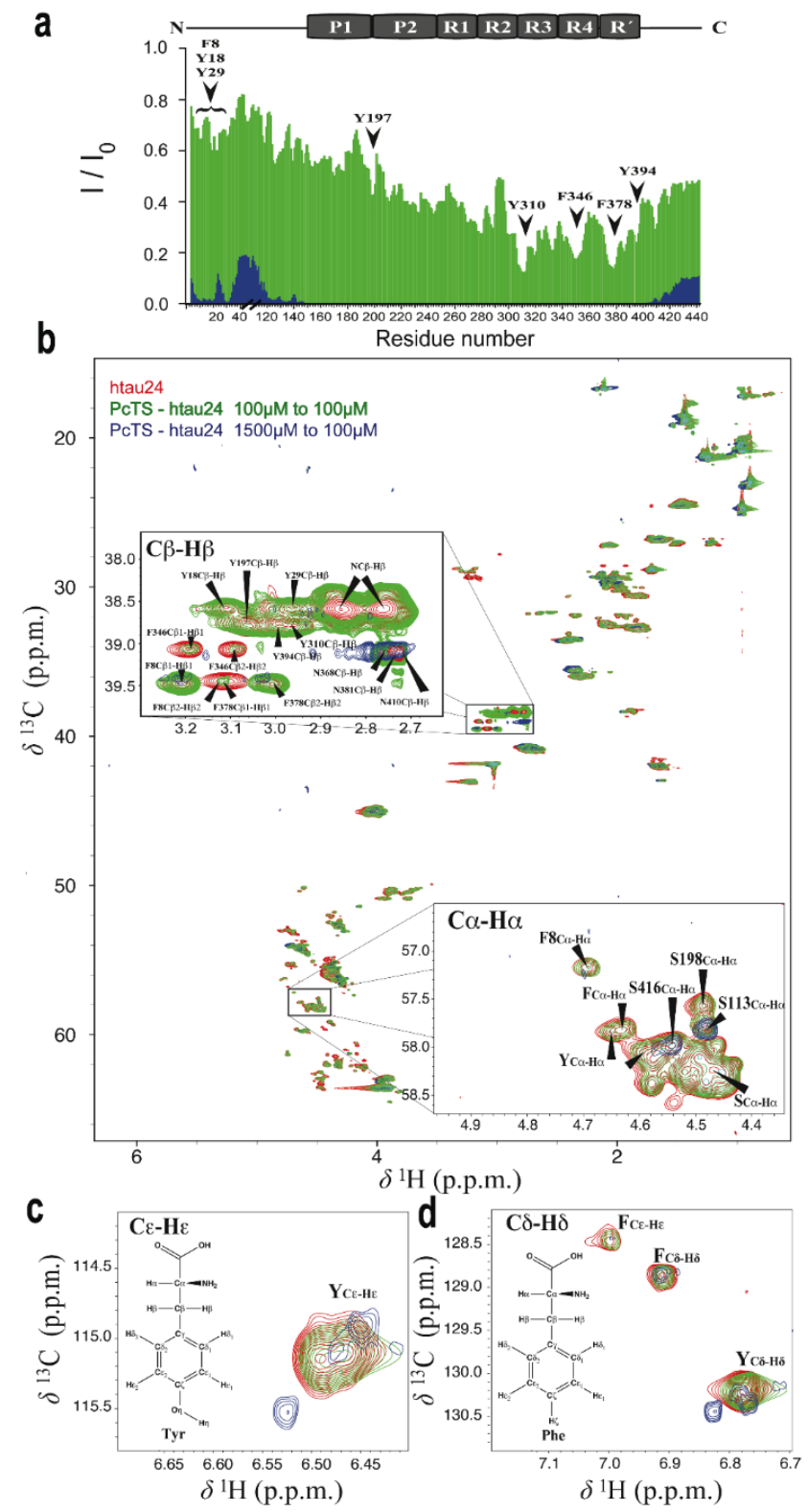

Figure S4: Interaction of PcTS with the shorter Tau isoform htau24.

(a) Ratios of NMR signal intensities observed in $2 \mathrm{D}{ }^{1} \mathrm{H}-{ }^{15} \mathrm{~N}$ HSQC of $100 \mu \mathrm{M}$ htau24 (in $50 \mathrm{mM}$ phosphate buffer $\mathrm{pH}$ 6.8) in the absence and presence of $100 \mu \mathrm{M}$ (green) and $1500 \mu \mathrm{M}$ (blue) PcTS. Residue numbers correspond to the sequence of htau40. The location of aromatic residues is indicated. Htau24 lacks the two negatively charged inserts (see domain organization on the top). (b) Overlay of the aliphatic regions from 2D ${ }^{1} \mathrm{H}-{ }^{13} \mathrm{C}$ HSQC spectra of htau24 in the absence (red) and presence of a 1 -fold (green) and 15-fold (blue) excess of PcTS. The aliphatic and aromatic (c and d) regions of the spectra show a strong decrease in the NMR signal intensities of the aromatic residues upon compound addition and their disappearance at large excess of compound. 


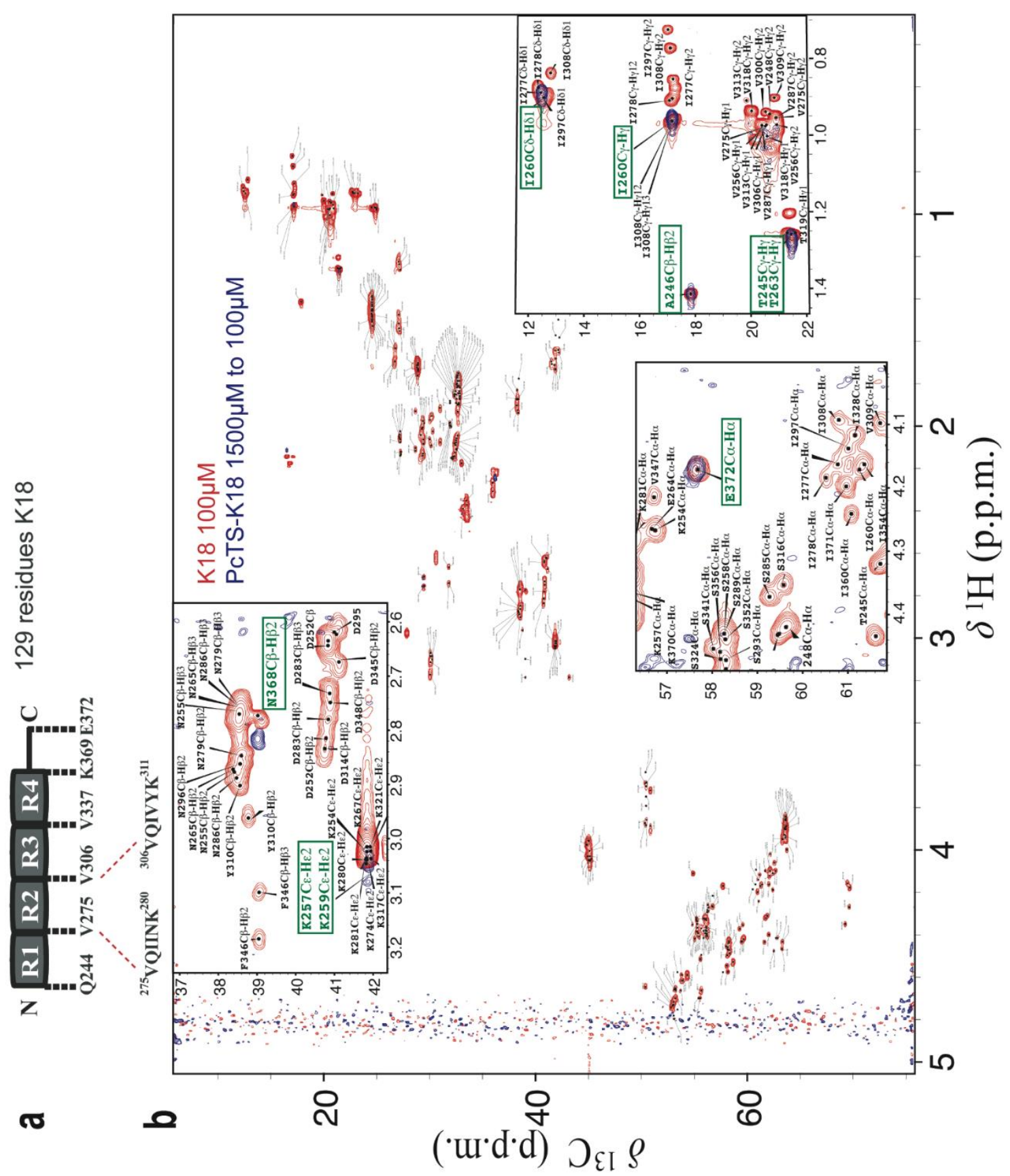

Figure S5: Interaction of PcTS with the Tau fragment K18 that comprises only the repeat domain of htau40.

(a) Domain organization of K18. The two hexapeptides at the N-terminal ends of repeats R2 and R3 are highlighted. (b) Superposition of $2 \mathrm{D}{ }^{1} \mathrm{H}-{ }^{13} \mathrm{C}$ HSQC spectra of $100 \mu \mathrm{M} \mathrm{K} 18$ without (red) and with 15 -fold (blue) excess of PcTS. The signals of all residues disappeared except for the highlighted resonances (green) that correspond to the flexible N-(A246, K257, K259, I260, and T263) and C- (N368 and E372) termini. 
a
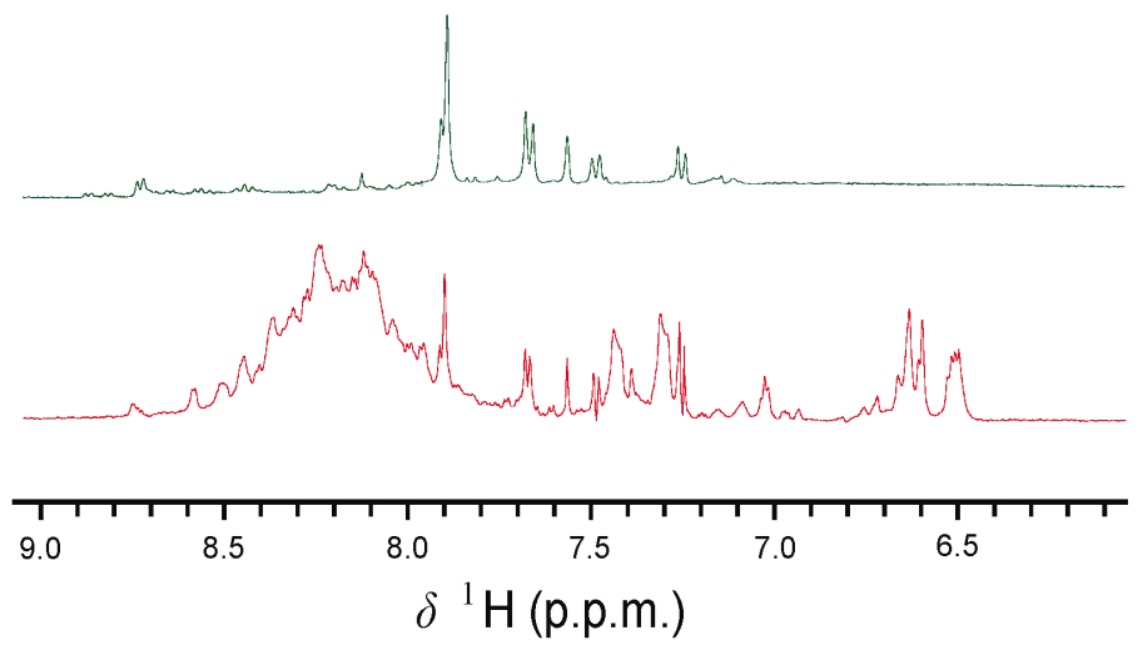

b

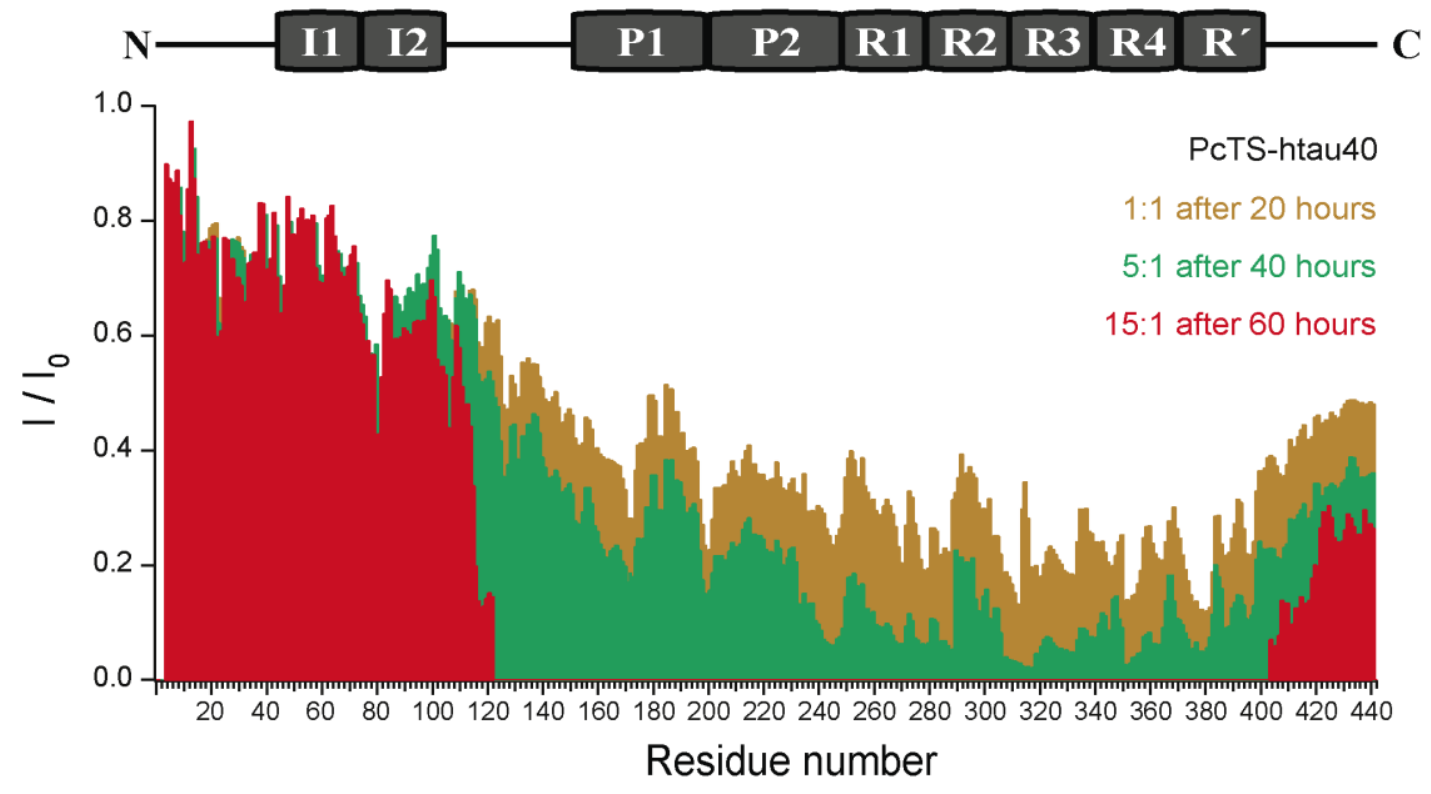

Figure S6: (a) 1D ${ }^{1} \mathrm{H}$ NMR of PcTS alone (green) and in the presence of htau40 at a PcTS:htau40 molar ratio of 15:1 (red). The concentration of PcTS and the experimental parameters were identical in the two measurements. The reduction of the PcTS signal in the presence of htau 40 suggests that about $40 \%$ of PcTS is incorporated into the Tau oligomers and therefore no longer visible in the $1 \mathrm{D}^{1} \mathrm{H}$ NMR spectrum. (b) NMR broadening profiles observed at a 10-fold lower concentration of htau40 $(10 \mu \mathrm{M})$ with the same PcTS:Tau molar ratio (1:1, yellow, 5:1 green, 15:1 red). The domain organization of htau40 is shown above. 
a

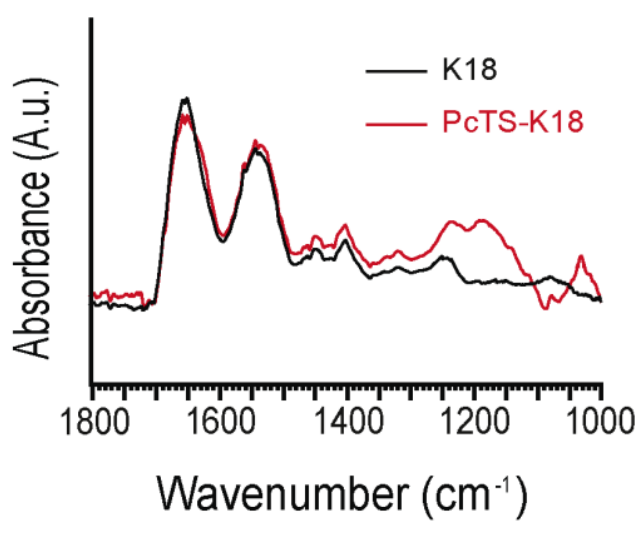

b

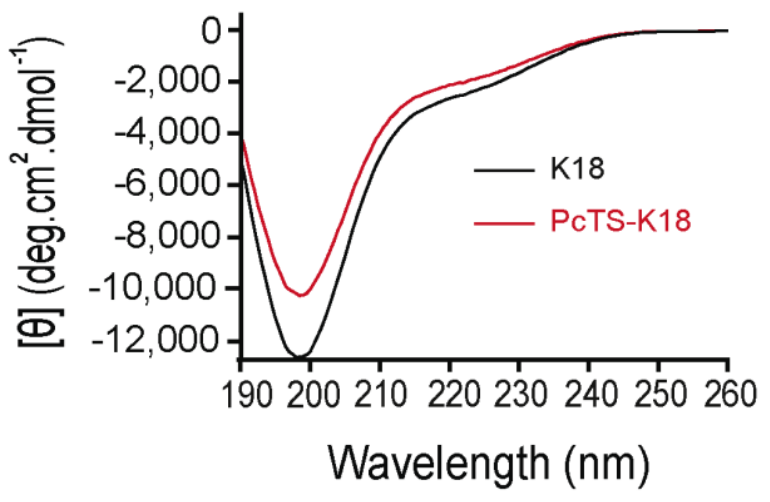

Figure S7: (a) FT-IR spectra of K18 in the absence (black) and presence of a 15-fold excess of PcTS (red). (b) Far UV-CD spectra of K18 in the absence (black) and presence of a 15-fold excess of PcTS (red). 


\section{Inhibition of Tau Filament Formation by Conformational Modulation}

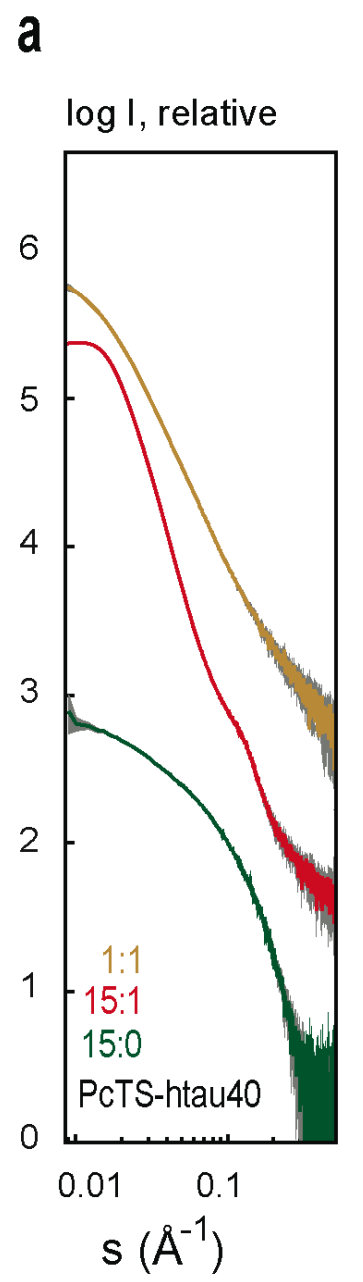

b

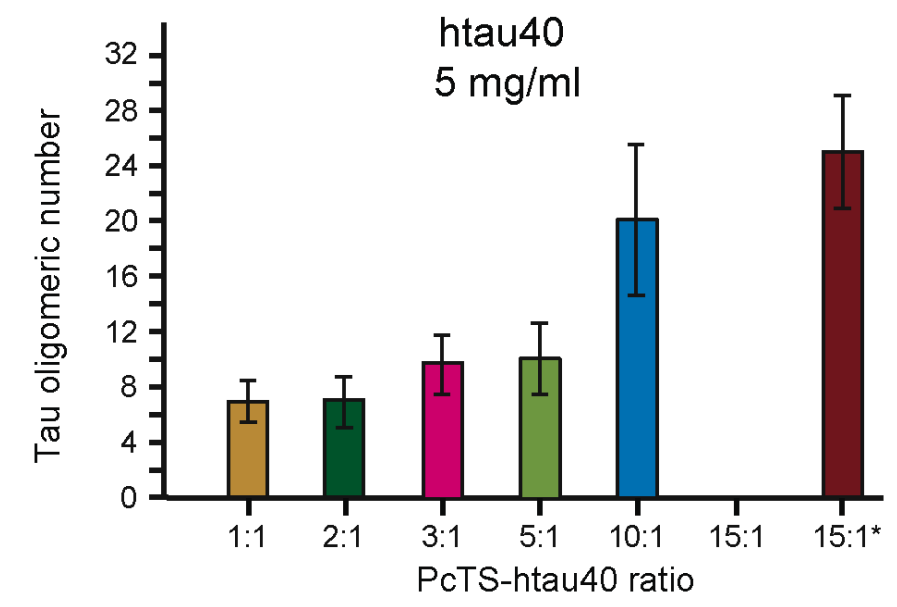

C

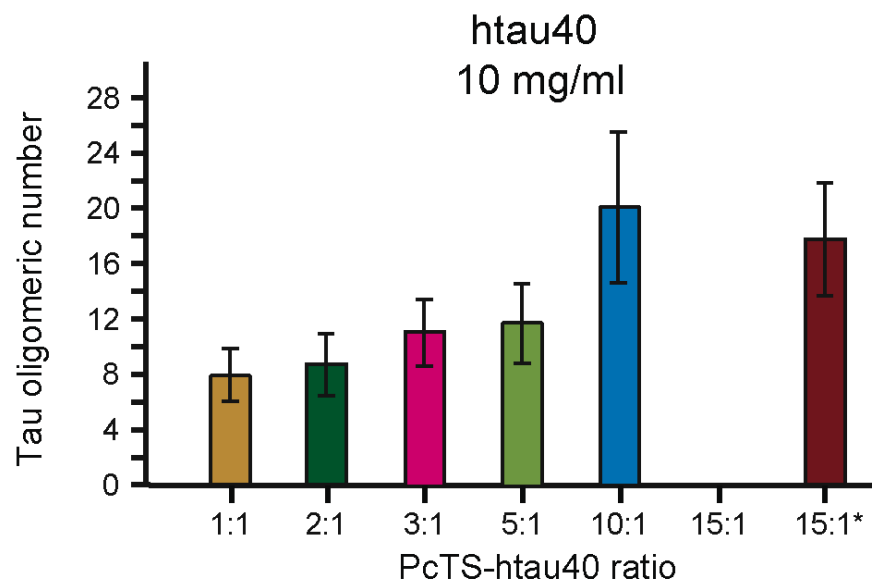

Figure S8: SAXS of PcTS-stimulated Tau oligomers.

(a) SAXS profile of PcTS alone (green) and PcTS-htau40 profiles (1:1 orange and 15:1 red). The three profiles have been scaled based on the PcTS concentration, i.e. such that the PcTS concentration is effectively the same for the three profiles. Note that the scattering intensity for PcTS alone is much smaller and does not substantially contribute to the scattering of the Tau oligomers. (b,c) Estimation of the number of Tau molecules incorporated into the oligomers at different PcTS:htau40 ratios containing 5 (b) or $10 \mathrm{mg} / \mathrm{ml}$ (c) htau40. Samples with a PcTS:htau40 ratio of 15:1 were filtered (using a centrifugal filter with $0.2 \mu \mathrm{m}$ cutoff) prior to the SAXS measurements. Error bars were estimated from the standard error of $\mathrm{I}_{0}$ variations between different concentrations of htau 40 but the same ratio of PcTS and accounting for the concentration of residual monomeric protein as determined by NMR (see Experimental Section for further information). 


\section{Inhibition of Tau Filament Formation by Conformational Modulation}
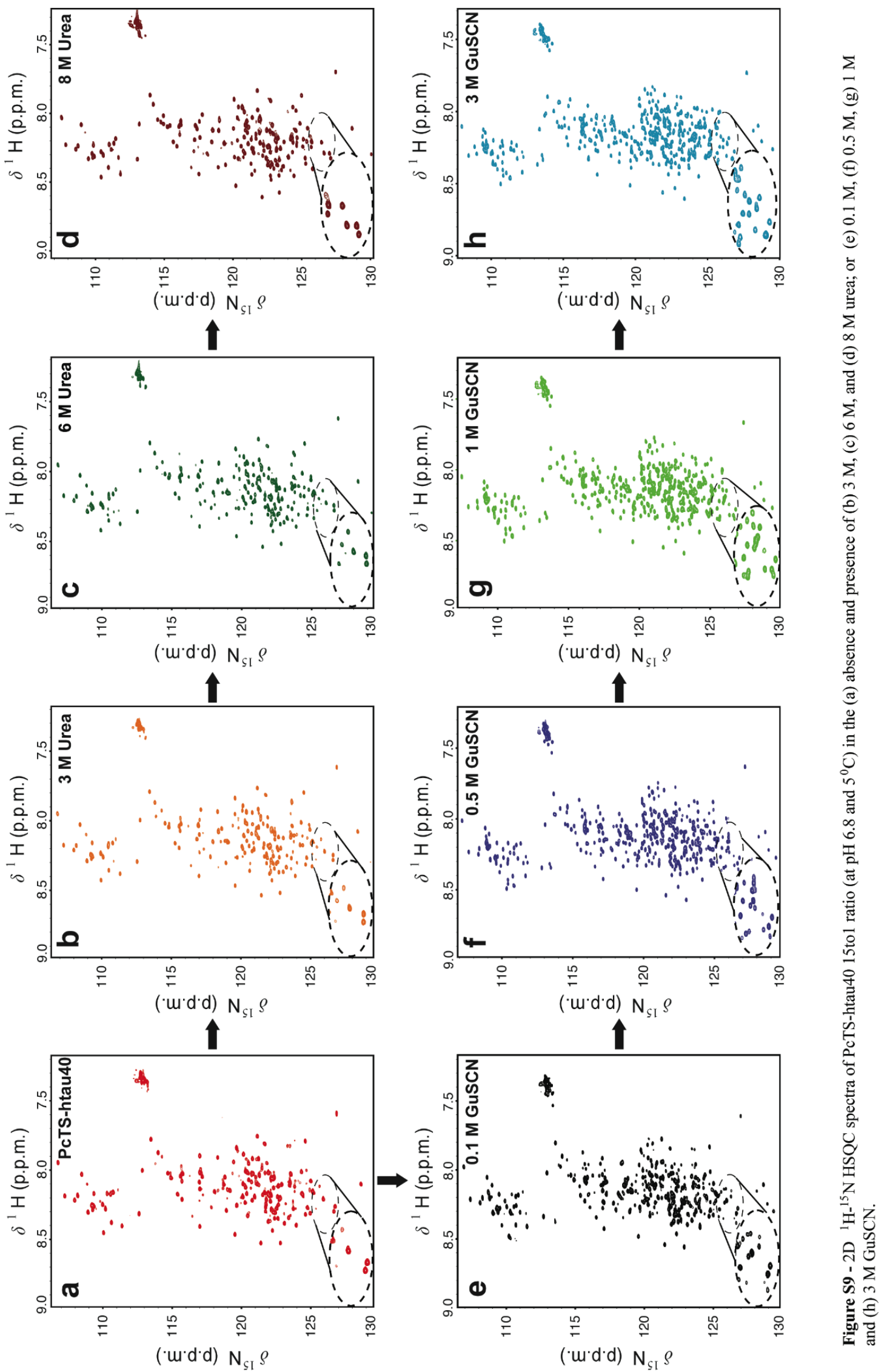


\section{Inhibition of Tau Filament Formation by Conformational Modulation}

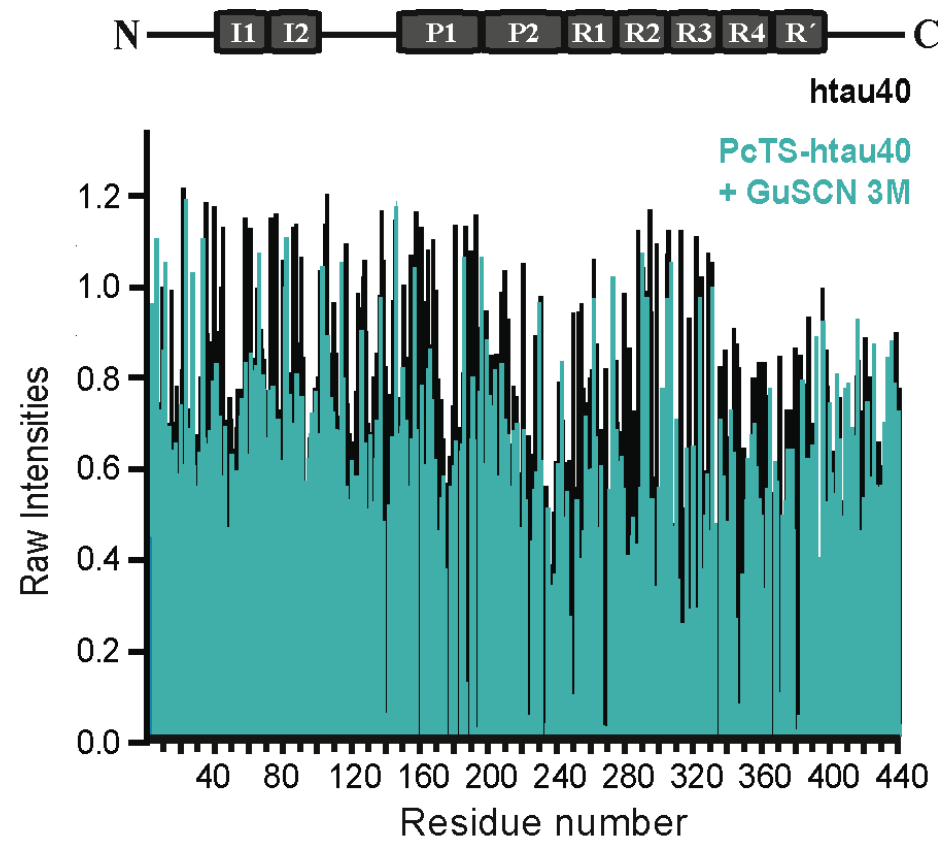

Figure S10: NMR signal intensities in $2 \mathrm{D}^{1} \mathrm{H}^{-15} \mathrm{~N}$ HSQC spectra of monomeric htau40 (black) and of htau40 in the presence of a 15-fold excess of PcTS and $3 \mathrm{M} \mathrm{GuSCN}$ (green). Intensities for all peaks are shown, both isolated and overlapped with other peaks and without 3-residue averaging. Signal intensities in the two spectra were normalized with respect to each other. 


\section{Mechanistic Basis of Phenothiazine-driven Inhibition}

\section{of Tau Aggregation}

Elias Akoury ${ }^{\mathrm{a}}$, Marcus Pickhardt ${ }^{\mathrm{b}}$, Michal Gajda ${ }^{\mathrm{a}}$, Jacek Biernat ${ }^{\mathrm{b}}$, Eckhard Mandelkow $^{\mathrm{b}, \mathrm{c}}$, and Markus Zweckstetter ${ }^{\mathrm{a}, \mathrm{d}}$

${ }^{a}$ Department for NMR-based Structural Biology, Max Planck Institute for Biophysical Chemistry, 37077 Göttingen, Germany. ${ }^{\mathrm{b}}$ German Center for Neurodegenerative Diseases (DZNE), LudwigErhard-Allee 2, 53175 Bonn, Germany. ${ }^{\mathrm{c}}$ CAESAR Research Center, Ludwig-Erhard-Allee 2, 53175 Bonn, Germany, ${ }^{\mathrm{d}}$ German Center for Neurodegenerative Diseases (DZNE), Göttingen, Germany.

* E. Akoury performed and analyzed NMR, DLS, CD and MS experiments and wrote the manuscript. 


\title{
3. Mechanistic Basis of Phenothiazine-driven Inhibition of Tau Aggregation
}

\begin{abstract}
Aggregation of the Tau protein is a key event in Alzheimer's disease and Tau aggregation inhibitors are important as potential drugs. Phenothiazines, such as methylene blue and its derivatives, have a unique mechanism, specifically modifying the Tau cysteine residues. The modification keeps Tau in a monomeric disordered conformation preventing the formation of filaments and their toxic precursors.
\end{abstract}

\section{INTRODUCTION}

Alzheimer's disease (AD) is the most widespread dementia syndrome showing progressive presence of abundant deposits of extracellular senile $\beta$-amyloid polypeptide $(\mathrm{A} \beta)$ plaques and intracellular neurofibrillary tangles (NFTs) consisting of Tau protein. ${ }^{[1,}$ ${ }^{2]}$ Tau protein is an intrinsically disordered protein that is abundant in neuronal axons where it promotes and stabilizes microtubule assembly. ${ }^{[3]}$ With progression of AD, Tau aggregates and accumulates into NFTs. ${ }^{[2]}$ As there is still no causative treatment or cure for $\mathrm{AD}$ and other tauopathies, Tau-based research aims to reveal the pathological consequences of amyloid formation and to implement new therapeutic strategies. In this effort, identification of inhibitors of tau aggregation as potential disease-modifying drugs and investigation of their mode of action play an important role. ${ }^{[4]}$

Methylene Blue (MB), a tricyclic phenothiazine also known as methylthionine hydrochloride, ${ }^{[5]}$ has a history of diverse medical applications stretching back over 100 years, including use for distinctive cellular targets. ${ }^{[6]} \mathrm{MB}$ has been shown to prevent Tau aggregation in vitro ${ }^{[6-8]}$ and to reduce the amount of Tau aggregates in a C. elegans model of Tau pathology. ${ }^{[9]}$ This treatment has relieved Tau-induced toxicity of treated worms. ${ }^{[9]}$ 


\section{Mechanistic Basis of Phenothiazine-driven Inhibition of Tau Aggregation}

Moreover, MB progressed to phase 2 clinical trials in human AD patients with promising results ${ }^{[10]}$ and was recently announced to enter a phase 3 clinical trials. ${ }^{[11]}$ Despite these positive effects, care must be exercised because $\mathrm{MB}$ and its derivatives azure $\mathrm{A}$ and $\mathrm{B}$ have toxic side effects at elevated concentrations. ${ }^{[12]}$ In model systems (e.g. Tau-expressing neuronal cells, C. elegans expressing Tau-V337M ${ }^{[9]}$ and zebra fish expressing Tau-P301S ${ }^{[13]}$ ) there are no detectable toxic effects at the concentrations used to inhibit Tau aggregation. Besides inhibition of Tau aggregation other modes of action for $\mathrm{MB}$ in Alzheimer's disease were proposed such as its antioxidant activity. ${ }^{[6]}$ Moreover, MB inhibits oligomerization of amyloid- $\beta$ peptide by promoting fibrillization and interferes with aggregation of prion protein. ${ }^{[14,15]}$ Mono- and di-N-demethylation of MB yield azure $\mathrm{B}$ and azure A, respectively (Scheme 1), which also have anti-aggregation and pharmacological effects. ${ }^{[8,12]}$ Herein we reveal a distinct mechanism of action of MB and its metabolites azure A and azure B in the inhibition of Tau aggregation. We show that the mechanism of Tau aggregation inhibition is based on the interplay of reduction/oxidation of the native cysteine residues of Tau. $\mathrm{MB}$ and its metabolites prevent the formation of filaments and their toxic precursors by retaining Tau in a monomeric disordered conformation.

The $\mathrm{IC}_{50}$ values reported for inhibition of Tau-aggregation by $\mathrm{MB}$ varies from approximately $2 \mu \mathrm{M}$ for four-repeat Tau to around $30 \mu \mathrm{M}$ for three-repeat Tau. ${ }^{[6,8,16]}$ To identify the residues of Tau that are essential for the interaction with MB, we used NMR spectroscopy. For increasing MB concentrations, changes in NMR signal position and intensity in two-dimensional ${ }^{1} \mathrm{H}^{-15} \mathrm{~N}$ heteronuclear single quantum coherence (HSQC) spectra of the 441-residue full-length Tau protein, htau40, were observed (Figure 1a and Figure S1 in the Supporting Information). 


\section{Mechanistic Basis of Phenothiazine-driven Inhibition of Tau Aggregation}<smiles>CNc1ccc2nc3ccc(N(C)C)cc3[o+]c2c1</smiles>

Azure B (oxidized form)

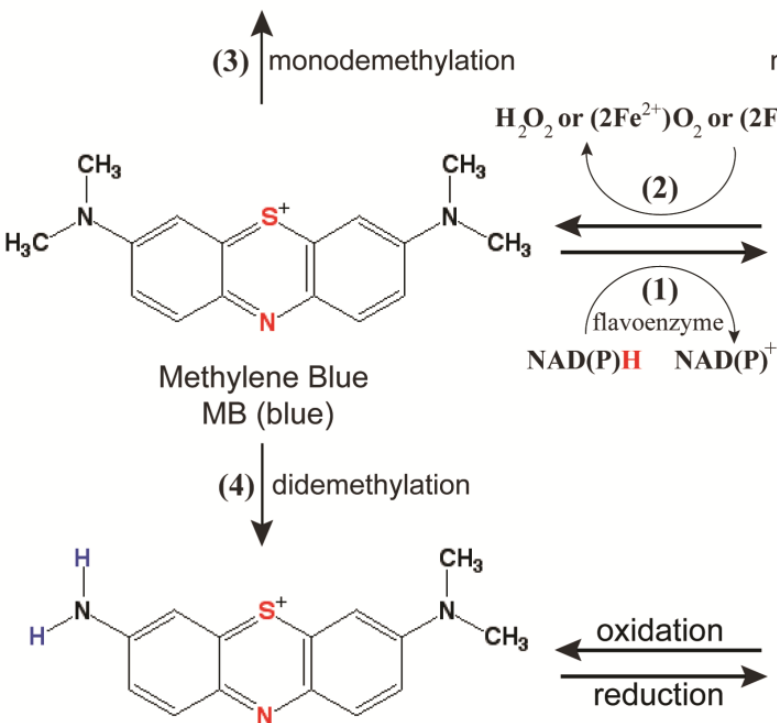

Azure A (oxidized form)<smiles>CNc1ccc2c(c1)Sc1cc(N(C)C)ccc1N2</smiles>

Azure B (reduced form)<smiles>CN(C)c1ccc2c(c1)Sc1cc(N(C)C)ccc1N2</smiles>

Leucomethylene Blue LMB (colorless)

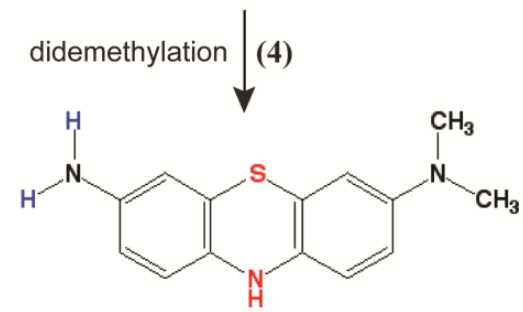

Azure A (reduced form)

Scheme 1: The reduction of MB yields leucoMB (1) and is regenerated in oxidizing conditions (2). Monoand di- $N$-demethylation yield azure B (3) and azure A (4), respectively.

Chemical shift changes were found in repeats R2 and R3 in the microtubule-binding domain and included the second hexapeptide ${ }^{306} \mathrm{VQIVYK}^{311}$ that is essential for aggregation of Tau. In addition, NMR signal perturbation was detected at the $\mathrm{N}$-terminus in proximity to the aromatic residues Y18 and Y29. Most apparent, however, was the strong broadening of residues in proximity to the two native cysteines of htau40, C291 and C322 (Figure 1a). Substitution of the two cysteines by alanine and glycine, respectively, abolished the NMR line-broadening in the repeat region (Figure 1b), and MB lost its inhibitory effect on Tau aggregation (Figure 2a,b, Figure S2). 


\section{Mechanistic Basis of Phenothiazine-driven Inhibition of Tau Aggregation}
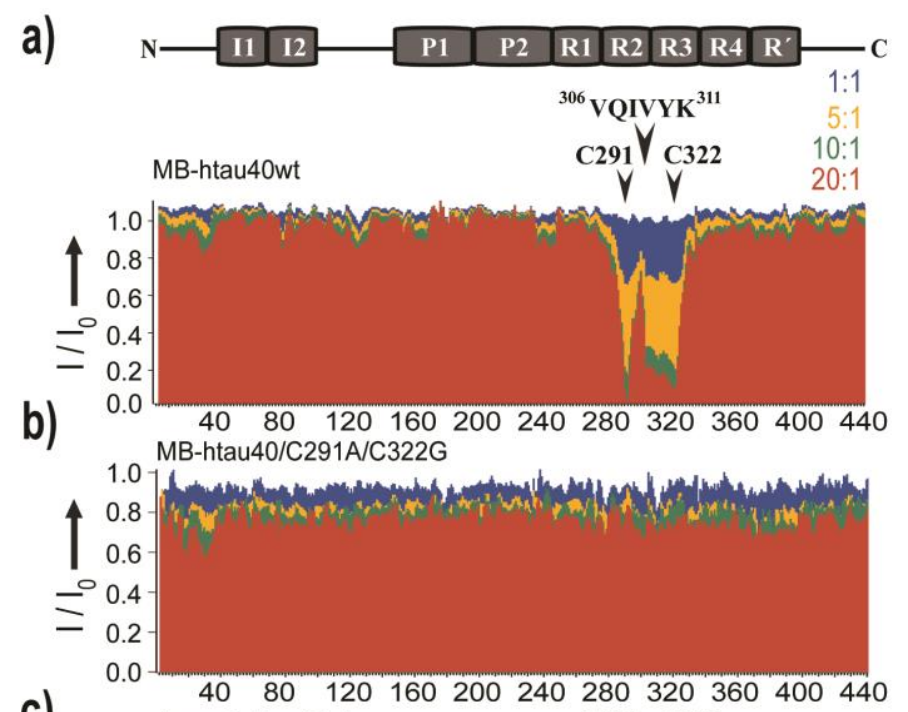

c)
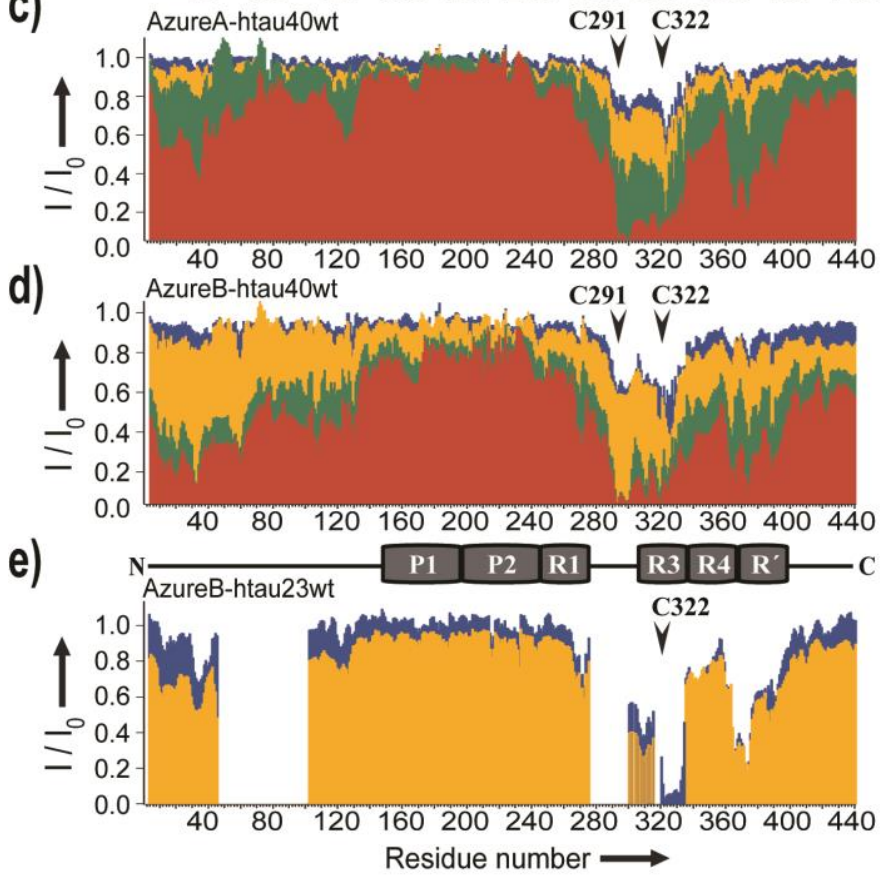

Figure 1: The Interaction of Methylene Blue with Tau. NMR intensity ratios $I / I_{0}$ ( $I=$ intensity of Tau resonances in presence of compound; $\mathrm{I}_{0}=$ peak intensities of free Tau) from $2 \mathrm{D}{ }^{1} \mathrm{H}-{ }^{15} \mathrm{~N}$ HSQC spectra of htau40 (a-d). Signal broadening $\left(\mathrm{I} / \mathrm{I}_{0}<1.0\right)$ is due to exchange of Tau between the free Tau conformations and those in the presence of the compound. (a) and (b) show the NMR broadening profile of wild-type and the cysteine-free variant of htau 40 at increasing concentrations of MB (see color-coded panel for ligand:protein ratios). The same titration analysis with wild-type htau 40 was conducted with azure A (c) and azure $\mathrm{B}(\mathrm{d})$. The domain organization of htau 40 is shown above $(\mathrm{I}=$ insert, $\mathrm{P}=$ proline-rich region, and $\mathrm{R}=$ pseudo-repeat). e) NMR signal broadening in ${ }^{1} \mathrm{H}-{ }^{15} \mathrm{~N}$ HSQC spectra of htau23 (lacking I1, I2 and R2) in the presence of azure B. All NMR experiments contained $100 \mu \mathrm{M}$ protein and $1 \mathrm{mM}$ DTT prepared in $50 \mathrm{mM}$ phosphate buffer, $\mathrm{pH} 6.8$. 


\section{Mechanistic Basis of Phenothiazine-driven Inhibition of Tau Aggregation}

a)
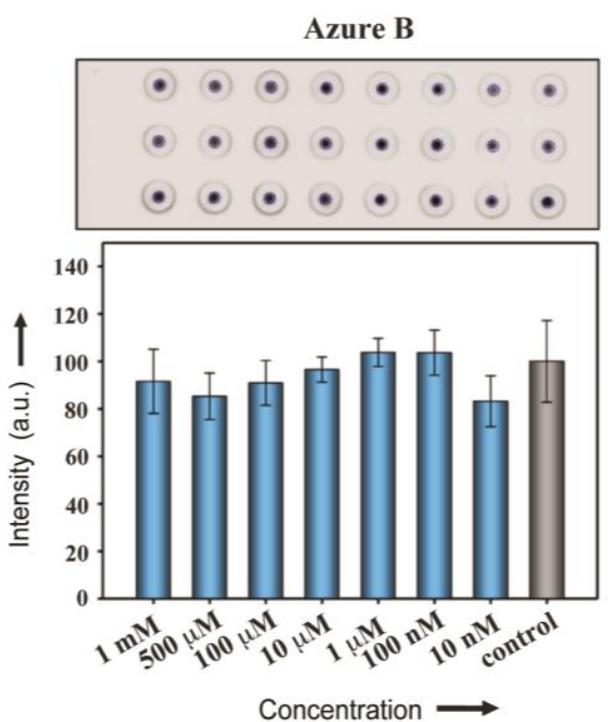

c)

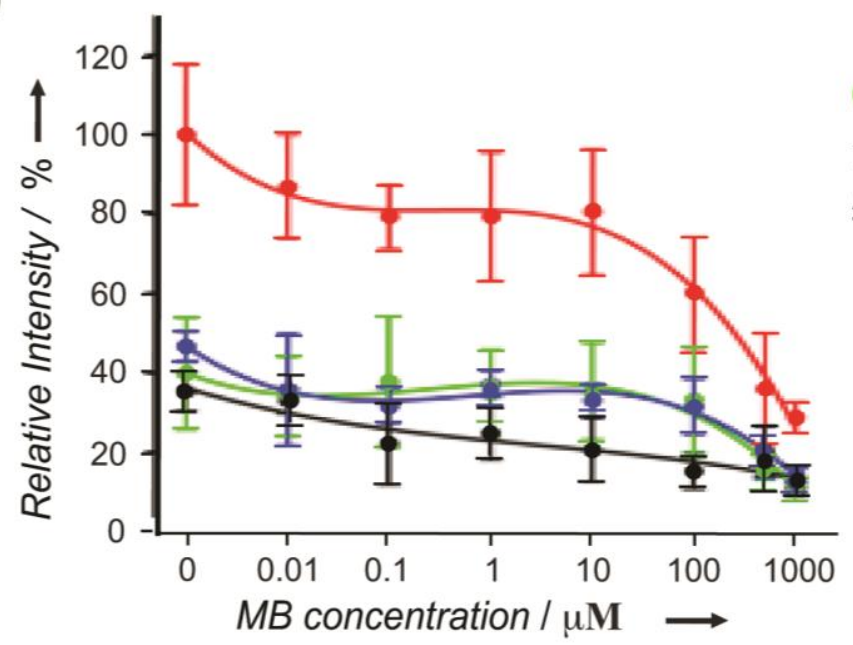

b)
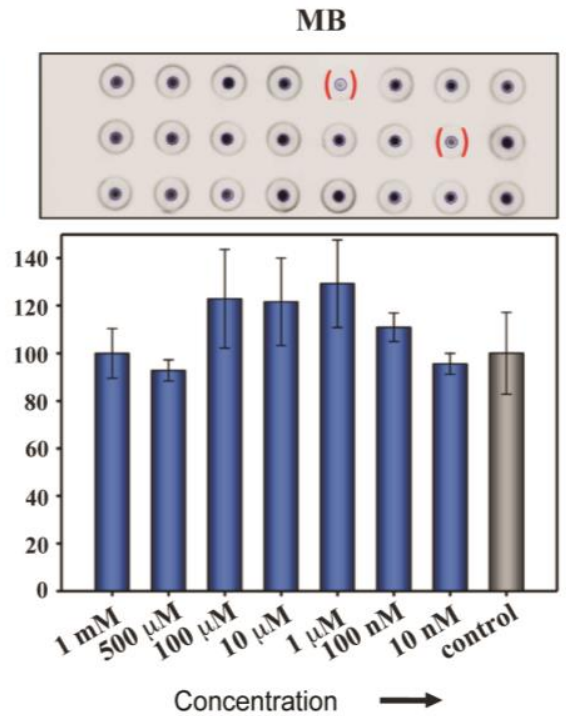

no DTT

\section{$0.5 \mathrm{mM}$ DTT}

$1.0 \mathrm{mM}$ DTT

$5.0 \mathrm{mM}$ DTT

Figure 2: a, b) Filter assay and immunological detection of htau40/C291A/C322A (see also Figure S2) with azure B (a) and MB (b). Note, that azure B and MB interfere with ThS fluorescence precluding the use of ThS fluorescence for detection of filament formation. c) Aggregation of the three-repeat Tau construct K19 in the presence of different concentrations of DTT and MB measured by a filter assay (see also Figure S5). Details on sample preparations are available as Supporting Information. The intensity refers to the amount of aggregated Tau. 
a)

b)

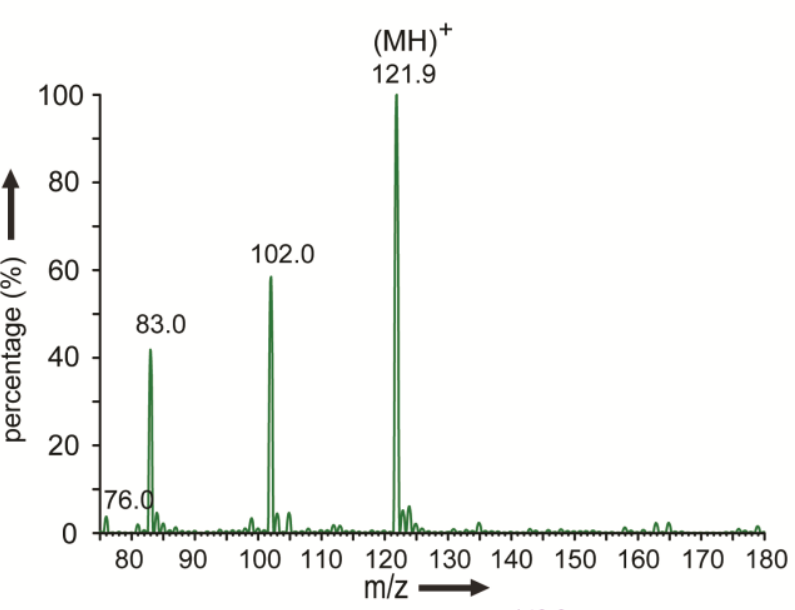

c) cys-SH

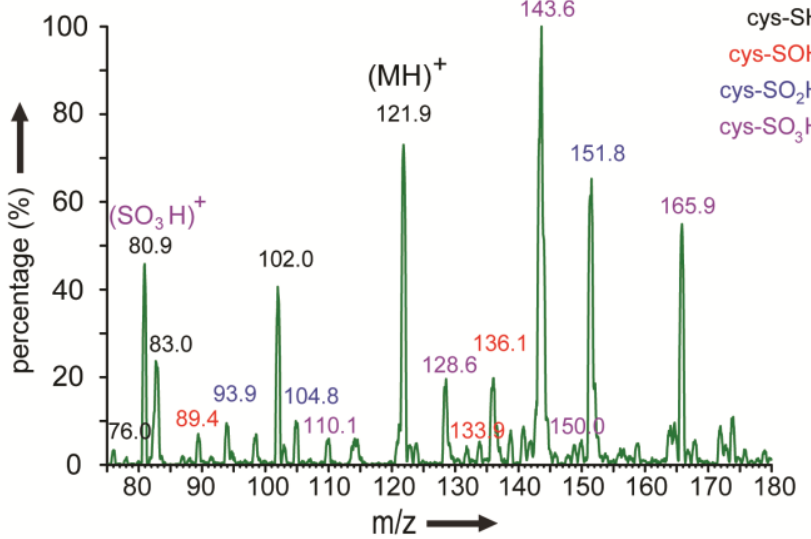

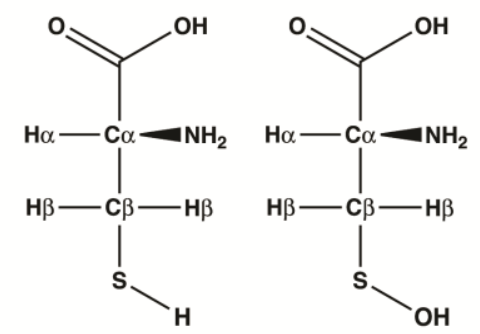

Cysteine

Cysteine sulfenic acid<smiles>C[C@H](N)[C@H](C)C(=O)O</smiles>

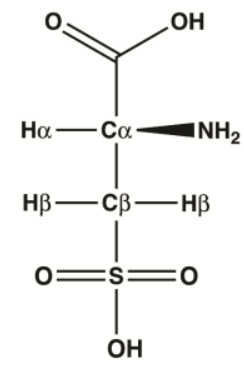

Cysteine sulfonic acid

Figure 3: a, b) ESI-MS spectra of the free amino acid cysteine in the absence (a) and presence of MB (b). The color-coded fragments correspond to different oxidation states of the sulfhydryl group as represented by their chemical structures shown in (c).

To obtain insight into the importance of the oxidation/reduction state of the cysteine residues of Tau, we added excess dithiothreitol (DTT) to the Tau-MB sample. Addition of DTT strongly decreased NMR signal broadening (see Figure S3), pointing towards MBinduced oxidation of the native cysteine residues of Tau. NMR and mass spectrometry demonstrated that MB modifies cysteines to sulfenic, sulfinic and sulfonic acids (Figures 3a-c and Figure S4). In addition, MB and its derivatives contain a central thiazine ring and are thus able to undergo oxidation-reduction reactions. 


\section{Mechanistic Basis of Phenothiazine-driven Inhibition of Tau Aggregation}

Although at neutral $\mathrm{pH}$ values the auto-oxidation rate of the reduced form of $\mathrm{MB}$, leucoMB, is very fast and leucoMB is not stable, dithiol compounds are able to produce leucoMB under quasiphysiological conditions (Figure S4). ${ }^{[17]}$ As we used 1 mM DTT to keep the native cysteine residues of Tau in a reduced state, an increasing fraction of MB was in the oxidized state when its concentration was increased during the titration.

NMR spectroscopy revealed a diminished binding capacity of MB to Tau in the presence of DTT (Figure S3). To answer the question if MB shows an inhibitory effect on Tau aggregation under reducing conditions, we performed a filter assay with the threerepeat Tau construct K19 (containing only one cysteine) in the presence of different DTT and $\mathrm{MB}$ concentrations. $10 \mu \mathrm{M}$ of $\mathrm{K} 19$ protein with $2.5 \mu \mathrm{M}$ heparin 3000 were incubated over $168 \mathrm{~h}$ with $0,0.5,1$ and $5 \mathrm{mM}$ DTT. The aggregation status of the MB-free sample was monitored via a Thioflavin S (ThS) assay (Figure S5). We observed diminished Tau aggregation in the presence of DTT. The ThS signal dropped to $36 \%$ of the DTT-free control. This is in line with a decrease of K19 dimer seeds by destruction of intermolecular disulfide bridges by DTT.

We tested the aggregation ability of K19 under different reducing conditions. MB was used in the range from 0 to $1 \mathrm{mM}$, aggregation was allowed to proceed for $168 \mathrm{~h}$, and the aggregation status was monitored with a filter assay (Figures $2 \mathrm{c}$ and S5). In the DTTfree control MB lowered the aggregation efficiency of K19 by about 70\%. With increasing amounts of DTT the inhibition of Tau aggregation was diminished. At $0.5 \mathrm{mM}$ and $1 \mathrm{mM}$ DTT the value dropped to approximately $30 \%$, and with $5 \mathrm{mM}$ DTT to $25 \%$. The experiments show that MB is able to inhibit the aggregation of three-repeat Tau under reducing conditions up to $5 \mathrm{mM}$ DTT. In living cells the reducing function is provided by gluthatione (GSH), an antioxidant preventing damage to important cell components. 


\section{Mechanistic Basis of Phenothiazine-driven Inhibition of Tau Aggregation}

For the proper function in the cell cytosol, GSH often reaches low millimolar levels. ${ }^{[18]}$ This situation suggests that the $5 \mathrm{mM}$ concentration of DTT chosen in our in vitro experiment was comparable to a relatively high cellular GSH concentration. It is known that the concentration of GSH declines with age and age-related neurodegenerative diseases. ${ }^{[19]}$ As a consequence the reducing potential in the cytosol will become weaker. Thus the lowering of the GSH concentration will favor the aggregation of Tau. Another factor favoring aggregation of Tau is oxidative stress that is elevated in neurons affected in neurodegenerative diseases and leads to a shift in the redox balance of GSH to less antioxidative values and thus lower antioxidative properties. ${ }^{[20]}$

As demethylation of $\mathrm{MB}$ is favoured with several in vitro and in vivo conditions, ${ }^{[5]}$ we probed the interaction of the didemethylated and monodemethylated derivatives of $\mathrm{MB}$, azure A and azure B (Scheme 1), with htau40. At low concentrations, azure A and B led to NMR signal broadening next to the two htau40 cysteines (Figure 1 c,d) which corresponds to their oxidation. However, at higher compound concentrations and in striking contrast to $\mathrm{MB}$, azure $\mathrm{A}$ and azure $\mathrm{B}$ also showed strong signal broadening in regions containing aromatic residues such as the $\mathrm{N}$-terminal tail and residues $360-400$ $\left({ }^{16} \mathrm{GTYG}^{19}, \quad{ }^{27} \mathrm{GGYT}^{30}, \quad{ }^{114}{ }^{\mathrm{LEDEAAGHVT}}{ }^{123}, \quad{ }^{362} \mathrm{HVPGGG}^{367}, \quad{ }^{371} \mathrm{IETH}^{374}\right.$ and ${ }^{386} \mathrm{TDHGAEIVYKS}^{396}$ ). A similar effect was observed for the three-repeat isoform of Tau, htau23, that contains only the single cysteine C322 and for which strong signal broadening was observed for C322 as well as ${ }^{362} \mathrm{HVPGGG}^{367}$ and ${ }^{371} \mathrm{IETH}^{374}$ (Figure 1e). The reason for binding of azure $\mathrm{A}$ and azure $\mathrm{B}$ and not $\mathrm{MB}$ to the aromatic residues of Tau, lies in the hydrophilic nature of the MB molecule, which has been suggested to be responsible for the finding that $\mathrm{MB}$ is not an efficient stain for normal blood cells in contrast to azure $\mathrm{B} .{ }^{[5]}$ Thus, upon demethylation of MB new modes of interaction and aggregation inhibition are 


\section{Mechanistic Basis of Phenothiazine-driven Inhibition of Tau Aggregation}

possible in line with the finding that azure $\mathrm{A}$ and $\mathrm{B}$ are 30 -fold more potent than $\mathrm{MB}$ in inhibition of Tau aggregation. ${ }^{[7]}$

The ${ }^{1} \mathrm{H}-{ }^{15} \mathrm{~N}$ HSQC of htau40 in the presence of MB, azure A and azure B retained its low dispersion and chemical shifts characteristic of a disordered protein. To obtain further insight into the Tau species that are stimulated by MB we performed circular dichroism (CD) and small angle X-ray scattering (SAXS) measurements. CD spectroscopy demonstrated that azure B did not induce detectable amounts of regular secondary structure in Tau (Figure 4a). SAXS showed that even at large excess of azure B, Tau is a highly disordered protein (Figures $\mathbf{4 b}$, c). The SAXS-derived radius of gyration, $\mathrm{R}_{\mathrm{g}}$ of $6.7 \pm 0.3$ $\mathrm{nm}$ (at five-fold excess of azure B), was comparable to that of monomeric Tau, ${ }^{[21]}$ demonstrating that the MB-Tau interaction does not result in the formation of Tau aggregates. Indeed, determination of the hydrodynamic radius by pulsed-field gradient $\mathrm{NMR}^{[22]}$ and dynamic light scattering (DLS) pointed to a slight compaction of the ensemble of Tau conformers that was released in the presence of excess DTT (Figure 4d). We conclude that $\mathrm{MB}$ and its derivatives azure $\mathrm{A}$ and $\mathrm{B}$ retain Tau in a monomeric aggregation incompetent state. 


\section{Mechanistic Basis of Phenothiazine-driven Inhibition of Tau Aggregation}

a)

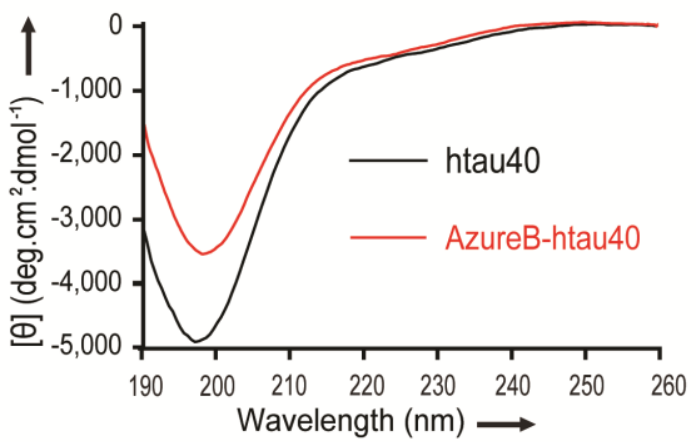

d)

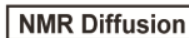

DLS

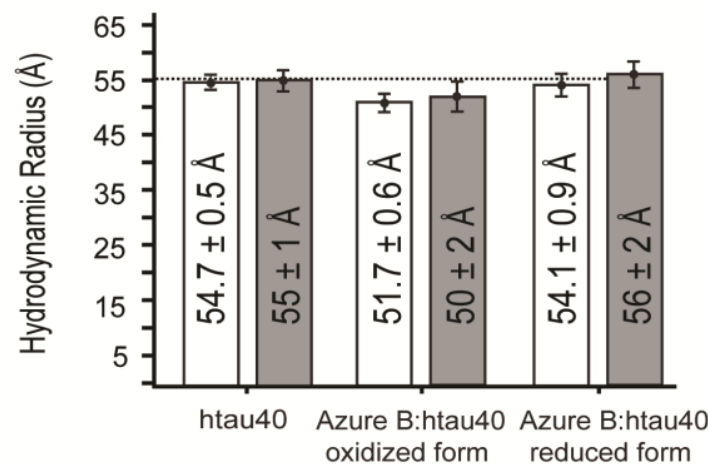

b)

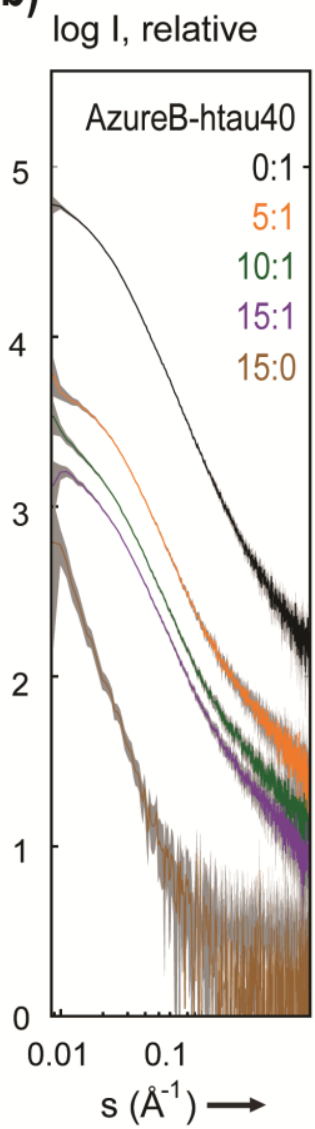

c) $\log I^{*} s^{2}$

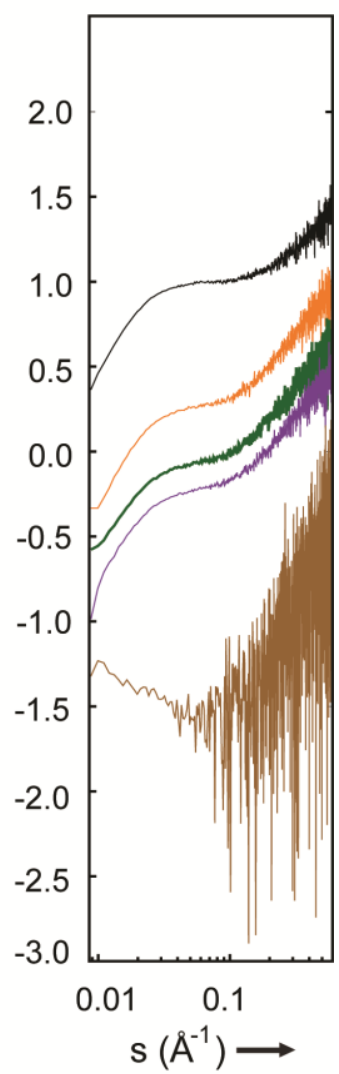

Figure 4: $\mathrm{MB}$ and its Metabolites Retain Tau in a Monomeric Disordered State. a) Far UV CD spectra of htau40 in the absence (black) and presence of azure B (20:1, red). b) SAXS profiles and c) Kratky plots of htau40 alone (black) and together with azure B (brown) at increasing ligand-protein ratios (color-coded panel). Curves are translated arbitrarily for viewing purposes. d) Hydrodynamic radius of htau40 in the absence and presence of a 20 -fold excess of azure B as determined by NMR and DLS, and after addition of $8 \mathrm{mM}$ DTT (right). Details on sample preparation are available as Supporting information.

An important aspect of aggregation inhibition is that it can potentially interfere with the physiological function of the protein. As Tau is a microtubule-associated protein, ${ }^{[23]}$ interference of phenothiazines with the ability of Tau to promote microtubule assembly might be detrimental in cells. However, azure A does not interfere with Tau-tubulin interaction when introduced at a 1000-fold excess with respect to Tau, ${ }^{[7]}$ and $\mathrm{MB}$ did not 


\section{Mechanistic Basis of Phenothiazine-driven Inhibition of Tau Aggregation}

influence Tau-promoted microtubule assembly. ${ }^{[8,24]}$ Thus, the aggregation incompetent monomeric conformation of Tau induced by $\mathrm{MB}$ and its derivatives is able to efficiently interact with microtubules.

Our data demonstrate that $\mathrm{MB}$ and its $\mathrm{N}$-demethylated derivatives azure $\mathrm{A}$ and azure B modify the two native cysteine residues of Tau to sulfenic, sulfinic and sulfonic acid. The modification of protein cysteine residues through reversible oxidation of cysteine sulfhydryl groups and the formation of sulfenic acids are a crucial regulatory event in biological systems. ${ }^{[25]}$ The nucleophilic character of the sulfhydryl group results in several distinct redox pathways and facilitates roles in electron donation, hydride-transfer reactions, and free radical reactions. Along these pathways, disulfide bridge formation is the most dominant chemical oxidation as it enhances structural stability. In case of Tau it was shown that intramolecular cross-linking of C291 and C322 in four-repeat isoforms of Tau (such as htau40) strongly inhibits PHF formation, while formation of intermolecular disulfide bond formation in three-repeat isoforms of Tau, e.g. htau23, promotes aggregation. ${ }^{[26]}$ In striking contrast, we demonstrate that modification of the cysteines to sulfenic, sulfinic and sulfonic acid converts both four-repeat and three-repeat Tau to an aggregation incompetent, monomeric state (Figures 3, 4): MB inhibits aggregation of both four-repeat Tau and three-repeat Tau with $\mathrm{IC}_{50}$ values of approximately $2 \mu \mathrm{M}^{[8,16]}$ and 30 $\mu \mathrm{M},{ }^{[6]}$ respectively. The lower $\mathrm{IC}_{50}$ value for three-repeat $\mathrm{Tau}$ is in line with the presence of a single cysteine residue, $\mathrm{C} 322$, that is, a single modification site, in comparison to the two cysteine residues of htau40. The specific modification of the cysteine residues is critical for aggregation inhibition as substitution of the cysteine residues by alanine did not interfere with filament formation (Figure S2), whereas it abolished the inhibitory effect of MB on Tau aggregation (Figures 2a, b). 


\section{Mechanistic Basis of Phenothiazine-driven Inhibition of Tau Aggregation}

In summary we have provided mechanistic insights into the inhibition of Tau aggregation by phenothiazines. Specific modification of the native cysteine residues retains Tau in a monomeric conformation preventing the formation of filaments and their toxic precursors. Demethylation of methylene blue establishes new interactions with Tau and enables additional means for modulation of Tau aggregation.

\section{EXPERIMENTAL SECTION}

Unlabelled and labeled wild-type and mutated human Tau proteins were expressed and purified as described in the literature. ${ }^{[27]} \mathrm{MB}$, azure A and B were purchased from MP Biomedicals (MP Biomedicals S.A. Heidelberg, Germany). NMR experiments were recorded at $5{ }^{0} \mathrm{C}$ on Bruker Avance $700 \mathrm{MHz}$ or Avance III $800 \mathrm{MHz}$ spectrometers. NMR samples contained ${ }^{15} \mathrm{~N}$-labeled Tau $(100 \mu \mathrm{M})$ in phosphate buffer $(50 \mathrm{mM} \mathrm{pH} 6.8)$, DTT $(1 \mathrm{mM})$, and $10 \%(\mathrm{v} / \mathrm{v}) \mathrm{D}_{2} \mathrm{O}$. SAXS data were collected at X33 at the European Molecular Biology Laboratory on DORIS III (DESY) at a wavelength of $1.5 \AA$ at $25^{\circ} \mathrm{C}$ using a Pilatus $1 \mathrm{M}$ photon counting detector. Samples were prepared in phosphate buffer $(50 \mathrm{mM})$ and contained htau40 (100 $\left.\mu \mathrm{M} ; 4.5 \mathrm{mg} \mathrm{mL}^{-1}\right)$ and DTT $(1 \mathrm{mM})$. Full details are available in the Supporting Information.

\section{AUTHOR INFORMATION}

\section{Corresponding Authors}

Markus.Zweckstetter@dzne.de 


\section{Mechanistic Basis of Phenothiazine-driven Inhibition of Tau Aggregation}

\section{ACKNOWLEDGMENTS}

E.A and M.P contributed equally to this work. We thank Dr. Andrei Leonov, Prof. Dr. Christian Griesinger, Dr. Eva-Maria Mandelkow, Prof. Dr. R. Heiner Schirmer, Dr. Bruno Bulic and Dr. Dmitri Svergun for stimulating discussions and Ilka Lindner for sample preparation. This work was supported by the Cluster of Excellence and DFG Research

Center "Nanoscale Microscopy and Molecular Physiology of the Brain", the MPG consortium Toxic Protein Conformation and the DFG (ZW 71/2-2, 3-2 to M.Z.).

\section{KEYWORDS}

Alzheimer disease, Tau, aggregation, inhibitor, NMR

\section{REFERENCES}

[1] J. Hardy, D. J. Selkoe, Science 2002, 297, 353-356.

[2] M. Goedert, M. G. Spillantini, Science 2006, 314, 777-781.

[3] D. G. Drubin, M. W. Kirschner, J Cell Biol 1986, 103, 2739-2746.

[4] B. Bulic, M. Pickhardt, B. Schmidt, E. M. Mandelkow, H. Waldmann, E. Mandelkow, Angew Chem Int Ed Engl 2009, 48, 1740-1752.

[5] M. Wainwright, L. Amaral, Trop Med Int Health 2005, 10, 501-511.

[6] R. H. Schirmer, H. Adler, M. Pickhardt, E. Mandelkow, Neurobiol Aging 2011, 32, 2325 e2327-2316.

[7] C. M. Wischik, P. C. Edwards, R. Y. Lai, M. Roth, C. R. Harrington, Proc Natl Acad Sci U S A 1996, 93, 11213-11218.

[8] S. Taniguchi, N. Suzuki, M. Masuda, S. Hisanaga, T. Iwatsubo, M. Goedert, M. Hasegawa, J Biol Chem 2005, 280, 7614-7623. 


\section{Mechanistic Basis of Phenothiazine-driven Inhibition of Tau Aggregation}

[9] C. Fatouros, G. J. Pir, J. Biernat, S. P. Koushika, E. Mandelkow, E. M. Mandelkow, E. Schmidt, R. Baumeister, Hum Mol Genet 2012, 21, 3587-3603.

[10]C. Wischik, R. Staff, J Nutr Health Aging 2009, 13, 367-369.

[11]C. Wischik, TauRX Therapeutics: Sept 10-th, 2012 Press release announcing the initiation of a global Phase 3 clinical trial in a type of Frontotemporal Dementia (FTD) also known as Pick's Disease.

[12]F. Culo, D. Sabolovic, L. Somogyi, M. Marusic, N. Berbiguier, L. Galey, Agents Actions 1991, 34, 424-428.

[13]F. van Bebber, D. Paquet, A. Hruscha, B. Schmid, C. Haass, Neurobiol Dis 2010, 39, 265-271.

[14]M. Necula, L. Breydo, S. Milton, R. Kayed, W. E. van der Veer, P. Tone, C. G. Glabe, Biochemistry 2007, 46, 8850-8860.

[15]P. Cavaliere, J. Torrent, S. Prigent, V. Granata, K. Pauwels, A. Pastore, H. Rezaei, A. Zagari, Biochim Biophys Acta 2012, 1832, 20-28.

[16]E. Chang, E. E. Congdon, N. S. Honson, K. E. Duff, J. Kuret, J Med Chem 2009, 52, 3539-3547.

[17]K. Buchholz, R. H. Schirmer, J. K. Eubel, M. B. Akoachere, T. Dandekar, K. Becker, S. Gromer, Antimicrob Agents Chemother 2008, 52, 183-191.

[18]P. M. Kidd, Alt Med Rev 1997, 2, 155-176.

[19]H. Liu, H. Wang, S. Shenvi, T. M. Hagen, R. M. Liu, Ann N Y Acad Sci 2004, 1019, 346-349.

[20] G. Perry, A. D. Cash, M. A. Smith, J Biomed Biotechnol 2002, 2, 120-123.

[21]E. Mylonas, A. Hascher, P. Bernado, M. Blackledge, E. Mandelkow, D. I. Svergun, Biochemistry 2008, 47, 10345-10353.

[22]E. O. Stejskal, J. E. Tanner, J Chem Phys 1965, 42, 288-292.

[23] M. D. Weingarten, A. H. Lockwood, S. Y. Hwo, M. W. Kirschner, Proc Natl Acad Sci U S A 1975, 72, 1858-1862. 


\section{Mechanistic Basis of Phenothiazine-driven Inhibition of Tau Aggregation}

[24]W. Li, J. B. Sperry, A. Crowe, J. Q. Trojanowski, A. B. Smith, 3rd, V. M. Lee, J Neurochem 2009, 110, 1339-1351.

[25]N. J. Kettenhofen, M. J. Wood, Chem Res Toxicol 2010, 23, 1633-1646.

[26] O. Schweers, E. M. Mandelkow, J. Biernat, E. Mandelkow, Proc Natl Acad Sci U S A 1995, 92, 8463-8467.

[27]M. D. Mukrasch, J. Biernat, M. von Bergen, C. Griesinger, E. Mandelkow, M. Zweckstetter, J Biol Chem 2005, 280, 24978-24986. 


\section{SUPPORTING INFORMATION}

\section{SUPPORTING TEXT}

Impact of removal of the two cysteines on Tau aggregation. Full-length Tau has a very low tendency to aggregate, consistent with its hydrophilic nature and high solubility. The influence of cysteines can be illustrated by the following experiments: The aggregation of wild-type htau40 is observed under non-reducing conditions (i.e. omission of DTT). The reaction was monitored with Thioflavin S (Figure S2a, blue line). In the course of 8 days there is no increase of the fluorescence signal, indicating little or no aggregation. Analysis of the endpoint sample by electron microscopy revealed some occasional short filaments (Figure S2b). By contrast, when the cysteine-less htau40-mutant was monitored over the same time period, it formed abundant amounts of bundled filaments (Figures S2a, red line and S2c). This can be rationalized in the following way ${ }^{[1]}$ : htau40 contains two cysteines (Cys291 and Cys322) which - in oxidative conditions - can form intra-molecular disulfide bridges that prevent dimerization and full-scale aggregation. But when the cysteine residues are replaced by alanine, aggregation can occur because the molecules are free to dimerize without the intra-molecular blocking conformation.

Next, we monitored the kinetics of aggregation of wild-type htau40 under nonreducing conditions in the presence of increasing amounts of MB by the filter assay (Figures S2d). [Note: MB interferes with the ThS fluorescence, which precludes the use of ThS fluorescence for filament detection.] The analysis showed small amounts of randomly aggregated material over the whole range of $\mathrm{MB}$ concentrations. 


\section{Mechanistic Basis of Phenothiazine-driven Inhibition of Tau Aggregation}

In the case of aggregation of wild-type htau40 under non-reducing conditions an inhibitory effect of MB cannot be detected because of the absence of free -SH groups (there is only minimal aggregation because of intra-molecular $\mathrm{S}-\mathrm{S}$ bridges in the compact monomer conformation, see $\left.{ }^{[1]}\right)$. The result of the comparable experiment with the cysteineless htau40-mutant also does not show any inhibitory effect of MB because of the lack of -SH groups. The noticeable aggregation of the htau40-mutant can be explained by the fact that this protein is able to dimerize and to form $\beta$-sheet stacking via hexapeptide motifs. In other words, intra-molecular disulfide bridges prevent aggregation (because they lock the molecule in a non-polymerizable state), inter-molecular disulfide bridges accelerate aggregation because they accelerate dimerization.

\section{SUPPORTING METHODS}

Proteins and Reagents. Unlabelled and ${ }^{15} \mathrm{~N}$-labelled wild-type and mutant human Tau proteins were expressed and purified as described previously. ${ }^{[2]}$ Methylene Blue (MB), azure A and azure B were purchased from MP Biomedicals (MP Biomedicals S.A. Heidelberg, Germany). Dithiothreitol DTT and Thioflavin S were purschased from Sigma (Sigma-Aldrich Chemie GmbH, Schnelldorf, Germany). ${ }^{13} \mathrm{C} /{ }^{15} \mathrm{~N}$ double-labelled cysteine was purschased from Cambridge Isotope Laboratories (Cambridge Isotope Laboratories, Andover, United States).

Quantification of aggregated Tau protein via filter-assay. $10 \mu \mathrm{M}$ of cysteinefree Tau protein (htau40/C291A/C322A) was incubated in the presence of BES buffer (20 $\mathrm{mM}, \mathrm{pH} 7.4), 25 \mathrm{mM} \mathrm{NaCl}, 2.5 \mu \mathrm{M}$ heparin 3000 , protease-inhibitor mix $(10 \mu \mathrm{g} / \mathrm{ml}$ leupeptin, $1 \mu \mathrm{g} / \mathrm{ml}$ aprotinin, $1 \mu \mathrm{g} / \mathrm{ml}$ pepstatin, and $1 \mathrm{mM}$ benzamidin). Methylene Blue 


\section{Mechanistic Basis of Phenothiazine-driven Inhibition of Tau Aggregation}

or Azure B were added in a concentration range from 0 to $1 \mathrm{mM}$ and the samples were incubated for 8 days at $37^{\circ} \mathrm{C}$. Tau aggregation was monitored in the compound free sample by Thioflavin $\mathrm{S}$ assay and electron microscopy. After incubation, $2 \%$ of the sample were filtered through an equilibrated nitrocellulose membrane $(\varnothing 0.45 \mu \mathrm{m}$; Biorad, Schleicher and Schüll Bioscience GmbH, Dassel, Germany) and washed two times with 40-fold PBS buffer volume (137 mM NaCl, $\left.2.7 \mathrm{mM} \mathrm{KCl,} 12 \mathrm{mM} \mathrm{Na} 2 \mathrm{HPO}_{4} / \mathrm{KH}_{2} \mathrm{PO}_{4}, \mathrm{pH} 7.4\right)$. the membrane was shaken for 15 minutes in PBS to remove air bubbles ouf of the nitrocellulose matrix. The amount of remaining aggregated Tau was quantified via immunological detection with the pan-Tau antibody K9JA (anti-rabbit-goat HRP). The secondary antibody is labeled with HRP and the measurement was performed via ImageQuant LAS4000 mini (GE Healthcare), detection via ECL system (GE Healthcare), and quantification via Aida Image Analyzer software.

Electrospray Ionization Mass Spectrometry. Samples of free $(100 \mu \mathrm{M})$ and MBcontaining (equimolar) ${ }^{13} \mathrm{C} /{ }^{15} \mathrm{~N}$ double-labelled cysteine amino acid (Cambridge Isotope Laboratories, Andover, United States) were dissolved in $20 \%$ acetonitrile / $0.2 \%$ formic acid-aqueous solutions and directly infused into a Thermofisher LTQ instrument via a syringe pump at $3 \mathrm{ml} / \mathrm{min}$. Spectra were acquired in the positive mode in the range of 60 to $600 \mathrm{~m} / \mathrm{z}$, under the following source conditions: voltage $3.8 \mathrm{kV}$, capillary temperature $275^{\circ} \mathrm{C}$; and processed with XCalibur.

NMR Spectroscopy. All NMR experiments were recorded at $5{ }^{0} \mathrm{C}$ on Bruker Avance $700 \mathrm{MHz}$ or Avance III $800 \mathrm{MHz}$ spectrometers equipped with cryogenic probes. NMR samples contained $100 \mu \mathrm{M}{ }^{15} \mathrm{~N}$-labelled protein in $50 \mathrm{mM}$ phosphate buffer $\mathrm{pH} 6.8$, $1 \mathrm{mM}$ DTT and $10 \%$ (v/v) $\mathrm{D}_{2} \mathrm{O}$ unless noted differently. $2 \mathrm{D}{ }^{1} \mathrm{H}-{ }^{15} \mathrm{~N}$ HSQC experiments were acquired using 600 complex points and 32 scans per increment with spectral widths 


\section{Mechanistic Basis of Phenothiazine-driven Inhibition of Tau Aggregation}

of $8389 \mathrm{~Hz}$ and $1844 \mathrm{~Hz}$ in the ${ }^{1} \mathrm{H}$ and ${ }^{15} \mathrm{~N}$ dimensions, respectively. Intensity ratio plots are reported with a 3-residues averaging window.

1D ${ }^{1} \mathrm{H}$ and $2 \mathrm{D}{ }^{1} \mathrm{H}-{ }^{13} \mathrm{C}$ NMR spectra of the amino acid cysteine $(100 \mu \mathrm{M}$ in the absence or presence of a 20 -folded excess of MB prepared in $50 \mathrm{mM}$ phosphate buffer, $\mathrm{pH}$ 6.8 and $10 \%(\mathrm{v} / \mathrm{v}) \mathrm{D}_{2} \mathrm{O}$, were acquired at $5{ }^{0} \mathrm{C}$ on a Bruker $400 \mathrm{MHz}$ spectrometer using $16 \mathrm{k}$ points, $1 \mathrm{k}$ scans with spectral widths of $4006 \mathrm{~Hz}$ (in case of $1 \mathrm{D}{ }^{1} \mathrm{H}$ NMR spectra) and 144 complex points, 16 scans per increment with spectral widths of $4006 \mathrm{~Hz}$ and $4024 \mathrm{~Hz}$ in the ${ }^{1} \mathrm{H}$ and ${ }^{13} \mathrm{C}$ dimensions, respectively (in case of $2 \mathrm{D}{ }^{1} \mathrm{H}-{ }^{13} \mathrm{C}$ NMR spectra).

NMR diffusion experiments were recorded on a Bruker Avance $600 \mathrm{MHz}$ spectrometer equipped with a cryogenic probe using a stimulated-echo based pulsed gradient spin-echo sequence incorporating the WATERGATE solvent suppression ${ }^{[3]}$ with optimized diffusion time and gradient pulses lengths. The gradient strength was linearly increased from $2 \%$ to $95 \%$ of the maximum gradient strength in 30 steps, where the 100 $\%$ gradient strength corresponds to $55.14 \mathrm{G} / \mathrm{cm}$. Each ${ }^{1} \mathrm{H}$ spectrum was recorded with 128 scans and 16K complex over a spectral width of $8992 \mathrm{~Hz}$. Several peaks in the aliphatic region of htau40 were selected and the intensities of their diffusion-based spin-echo attenuation were extracted to determine an average diffusion coefficient. Stokes radii were calculated from the apparent diffusion coefficients of htau40 referenced to a selection of protein standards (cytochrome C $12.4 \mathrm{KDa}, 17.8 \AA$ A; lysozyme 14.3 KDa, $20.5 \AA$; myoglobin $18 \mathrm{KDa}, 21.2 \AA$; and ovalbumin $45 \mathrm{KDa}, 30.5 \AA$; all prepared at concentrations of $100 \mu \mathrm{M}$ in $50 \mathrm{mM}$ phosphate buffer containing $1 \mathrm{mM}$ DTT and $\left.10 \%(\mathrm{v} / \mathrm{v}) \mathrm{D}_{2} \mathrm{O}\right)$.

Dynamic Light Scattering. DLS measurements were performed at $5{ }^{0} \mathrm{C}$ using a DynaPro Titan/temperature controlled microsampler (Wyatt Technologies Corporation). Samples (40 $\mu \mathrm{M}$ of htau40 in $50 \mathrm{mM}$ phosphate buffer, pH 6.8 and $1 \mathrm{mM}$ DTT inserted in 


\section{Mechanistic Basis of Phenothiazine-driven Inhibition of Tau Aggregation}

$50 \mu \mathrm{l}$ flow cells) were illuminated by a $25 \mathrm{~mW}, 780 \mathrm{~nm}$ solid-state laser, and the intensity of $90^{\circ}$ angle scattered light was measured at $4 \mu$ s intervals by a solid-state avalanche photodiode. Measurements were repeated three times using freshly prepared samples to confirm the reproducibility of the results, and the average values with their standard deviations were analyzed and reported by the Dynamics 6.7.7.9 software package.

Circular Dichroism. Samples contained $10 \mu \mathrm{M}$ htau40 in $50 \mathrm{mM}$ phosphate buffer pH 6.8 and 1 mM DTT. CD spectra were acquired at room temperature using a 1-mm path cell cuvette, on a Chirascan CD spectrometer (Applied Photophysics Limited) over the range covering 190 to $250 \mathrm{~nm}$ and a $1 \mathrm{~nm}$ band width and a scanning speed of $20 \mathrm{~nm} / \mathrm{min}$. Spectra were averaged over five scans. The measurements were repeated three times with freshly prepared samples to confirm the reproducibility of the results. Data are expressed in terms of the mean residual ellipticity $(\theta)$ in $\mathrm{deg} \mathrm{cm}^{2} \mathrm{dmol}^{-1}$.

Small Angle X-ray Scattering. SAXS Data were collected at X33 at the European Molecular Biology Laboratory on DORIS III (DESY) at a wavelength of $1.5 \AA$ at $25^{\circ} \mathrm{C}$ using a Pilatus $1 \mathrm{M}$ photon counting detector. Each sample contained $87 \mu \mathrm{M}(4 \mathrm{mg} / \mathrm{m})$ of htau40 in $50 \mathrm{mM}$ phosphate buffer, $\mathrm{pH} 6.8$ and $1 \mathrm{mM}$ DTT, and was exposed to 8 frames

of 15 seconds. Data were analyzed by ATSAS 2.3. ${ }^{[4]}$ The maximum diameter was estimated by indirect fourier transformation. 


\section{SUPPORTING FIGURES}

a)

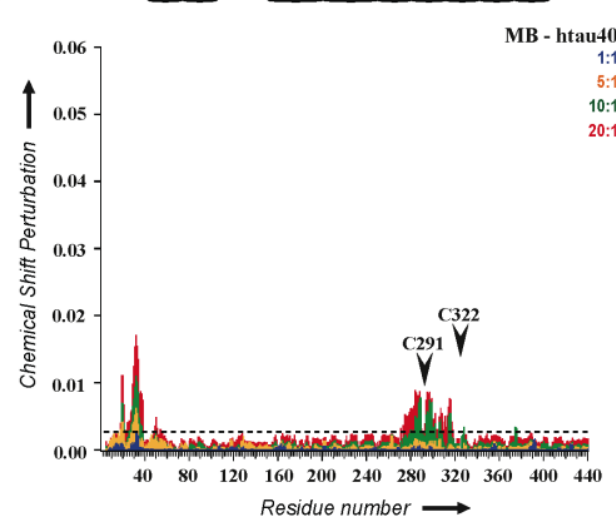

c)

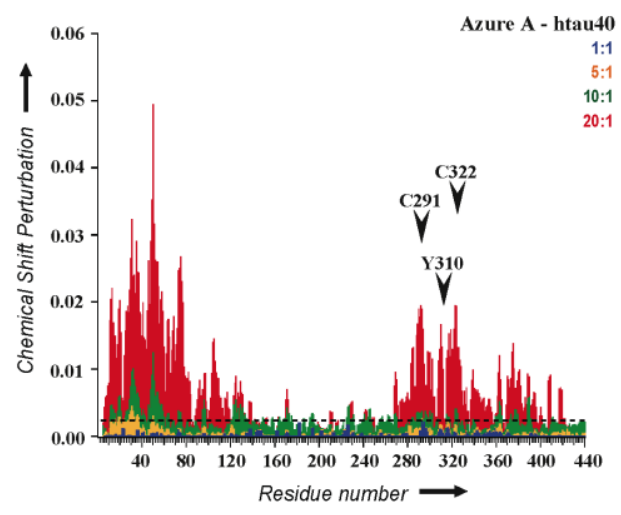

b)

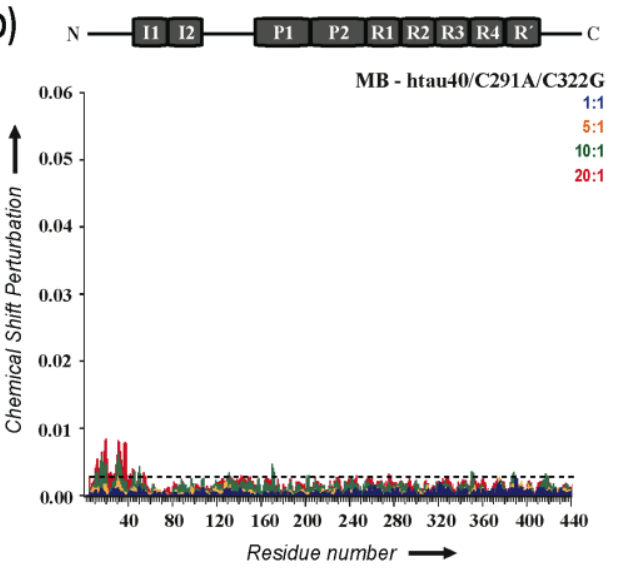

d)

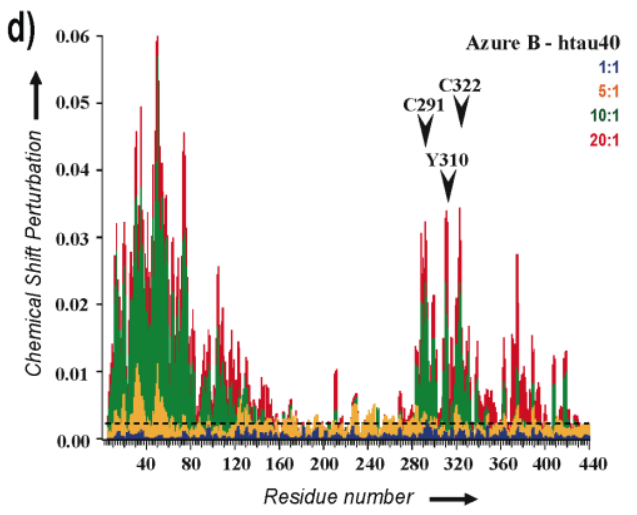

e)

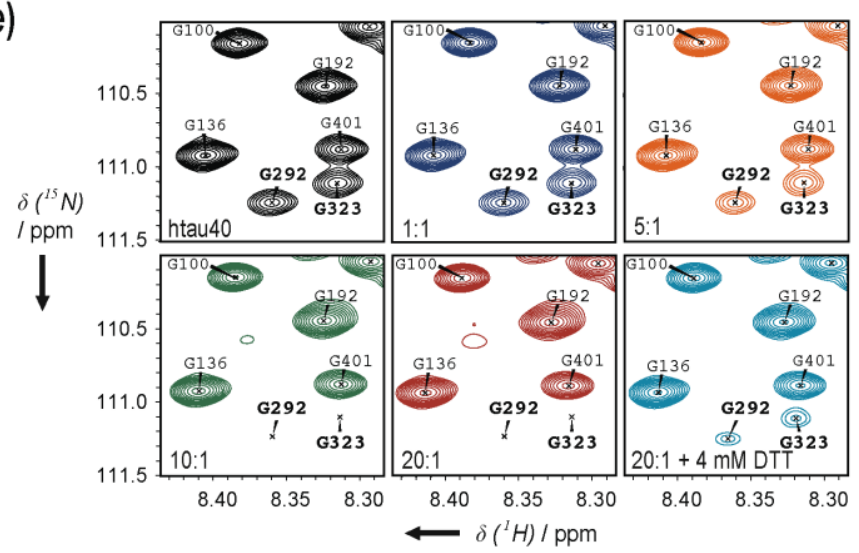

Figure S1. Chemical shift deviations $\left(\Delta \delta_{1 \mathrm{H} / 15 \mathrm{Ntit}}-\Delta \delta_{1 \mathrm{H} / 15 \mathrm{Nref}}\right)$ observed in $2 \mathrm{D}{ }^{1} \mathrm{H}-{ }^{15} \mathrm{~N}$ HSQC NMR spectra of htau40 wild-type and mutants at increasing compound concentrations: a, b) MB, c) azure A, and d) azure B. Averaged, normalized chemical shift deviations were calculated according to $\Delta \delta_{\mathrm{AV}}=\left[0.5\left(\delta_{\mathrm{Htit}}-\delta_{\mathrm{Hwt}}\right)^{2}+0.02\left(\delta_{\mathrm{Ntit}}-\delta_{\mathrm{Nwt}}\right)^{2}\right]^{1 / 2}$. Dotted black lines correspond to thresholds of chemical shift perturbations of $0.0025 \mathrm{ppm}$. e) Selected regions of $2 \mathrm{D}^{1} \mathrm{H}-{ }^{15} \mathrm{~N}$ HSQC of htau40 at increasing concentrations of MB, and after further addition of $4 \mathrm{mM}$ DTT (light blue). 


\section{Mechanistic Basis of Phenothiazine-driven Inhibition of Tau Aggregation}

a)

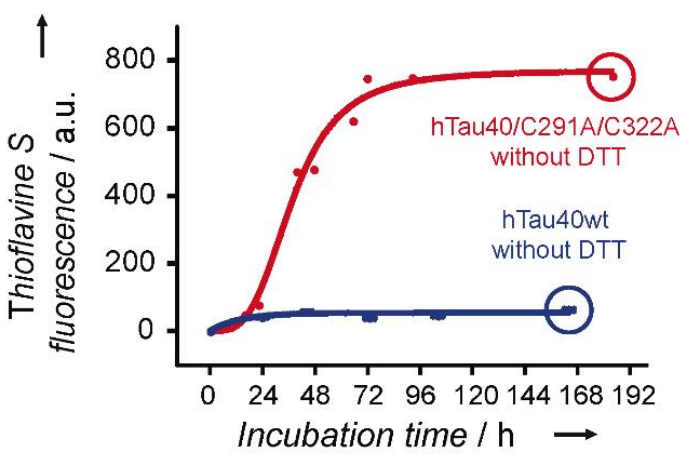

b)

c)
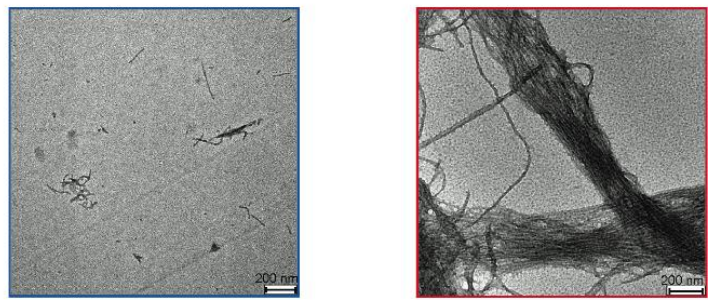

d)
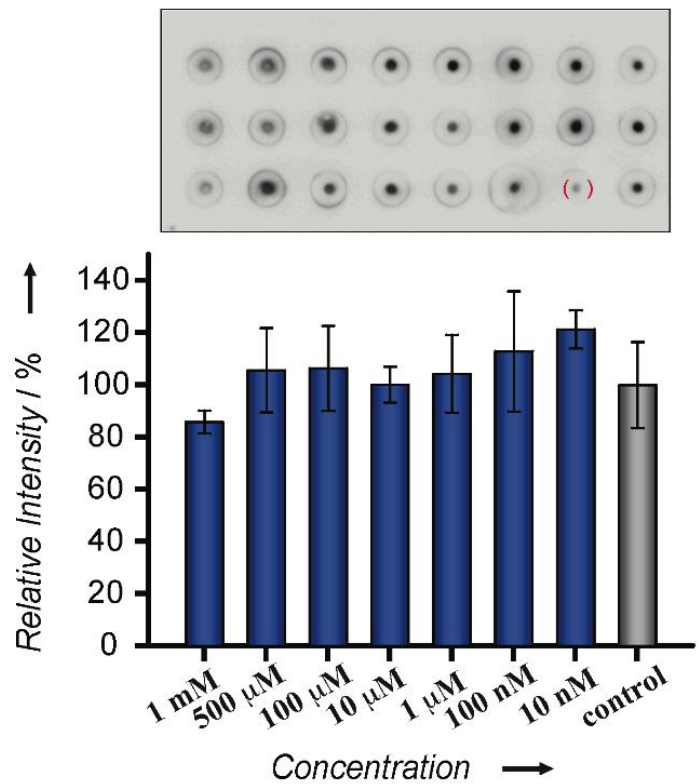

Figure S2. Impact of removal of the two native cysteines on Tau aggregation. a) Aggregation curve of wildtype htau40 (blue line) compared with the cysteine-less mutant htau40/C291A/C322A (red line) under nonreducing conditions without $\mathrm{MB}$ treatment monitored by a Thioflavin $\mathrm{S}$ assay. b, c) Endpoint samples (after $168 \mathrm{~h}$ for wild-type htau40, blue circle and after 192h for htau40/C291A/C322A, red circle) were monitored by electron microscopy to show the amount and shape of aggregated Tau protein. d) Filter assay of aggregated wild-type htau40 in the presence of increasing amounts of MB (0 to $1 \mathrm{mM}$ ) (see Supporting Text for additional information). 


\section{Mechanistic Basis of Phenothiazine-driven Inhibition of Tau Aggregation}

a)

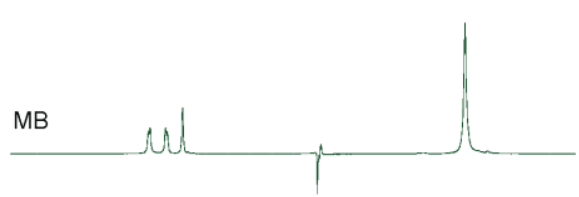

b)
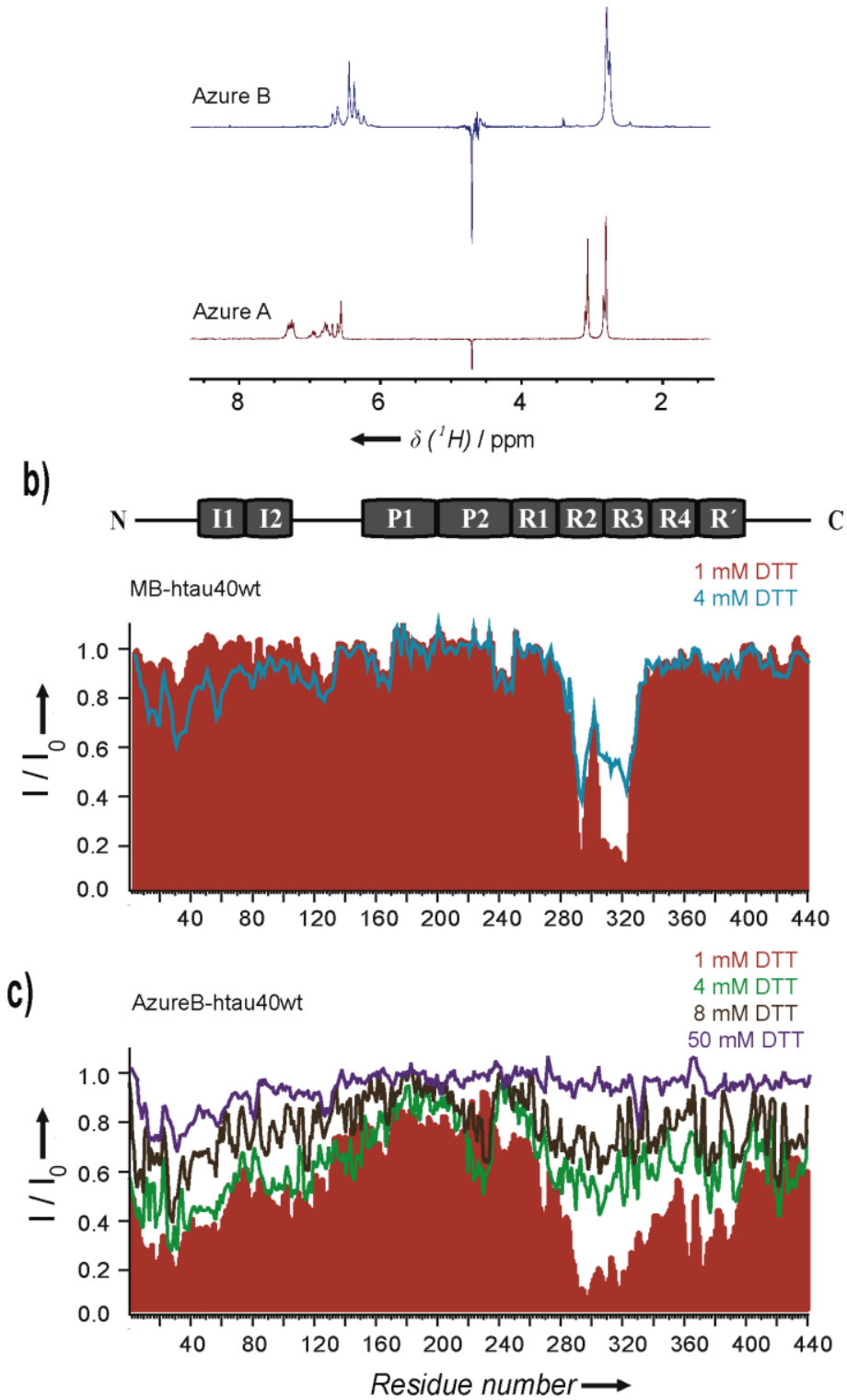

Figure S3. Interplay between oxidized and reduced forms of MB and azure B. a) $1 \mathrm{D}^{1} \mathrm{H}$ NMR spectra of $\mathrm{MB}$, azure B and azure A. b) NMR signal intensity ratios in $2 \mathrm{D}{ }^{1} \mathrm{H}-{ }^{15} \mathrm{~N}$ HSQC of htau 40 for MB-htau40 20:1 in the presence of $1 \mathrm{mM}$ (red) and of $4 \mathrm{mM}$ DTT (light blue). c) NMR signal intensity ratios in $2 \mathrm{D}{ }^{1} \mathrm{H}-$ ${ }^{15} \mathrm{~N}$ HSQC of htau40 for azureB-htau40 20:1 in presence of $1 \mathrm{mM}$ (red), $4 \mathrm{mM}$ (green), $8 \mathrm{mM}$ (brown) and $50 \mathrm{mM}$ DTT (purple). 


\section{Mechanistic Basis of Phenothiazine-driven Inhibition of Tau Aggregation}

a)

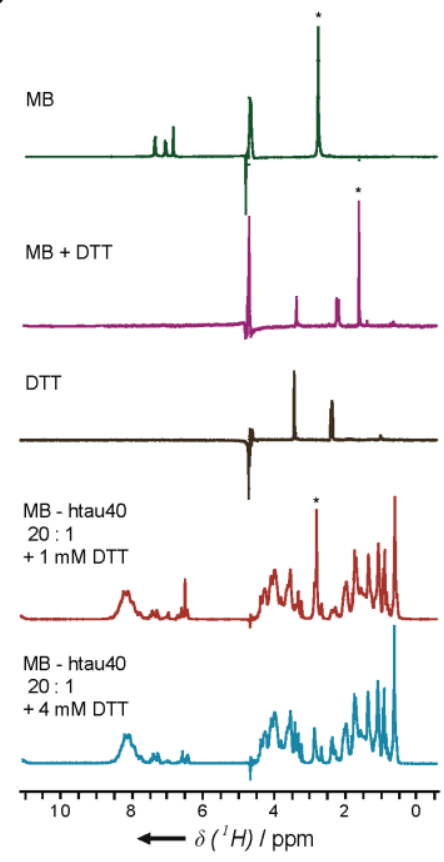

b)

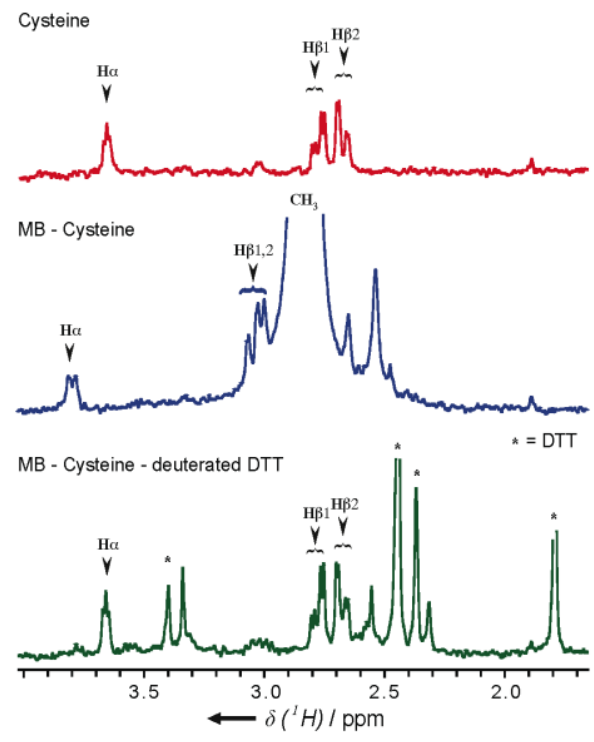

c)

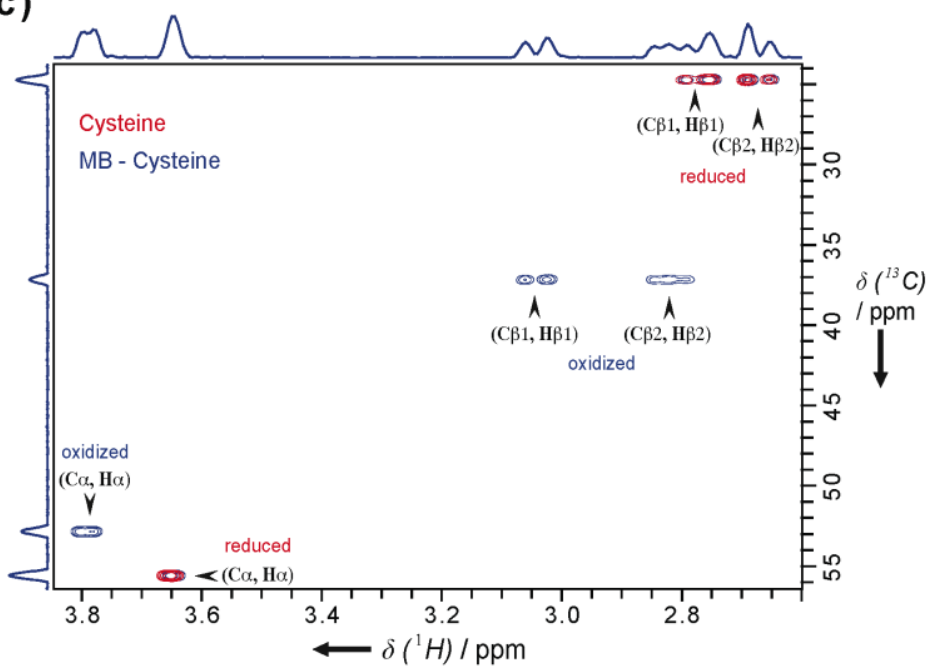

Figure S4. Reversible modification of cysteine by MB. a) 1D ${ }^{1} \mathrm{H}$ NMR spectra of selected samples to highlight the redox reaction. The oxidized form of MB is reduced in the presence of excess DTT. Upon increasing the concentration of deuterated DTT from 1 to $4 \mathrm{mM}$ in the MB-htau40 sample, the concentration of oxidized MB is decreased. Note that this was also clearly visible by the change in color of the NMR sample. The sample color changed from blue (oxidized MB) to transparent (leucoMB). b) $1 \mathrm{D}{ }^{1} \mathrm{H}$ NMR spectra of the free amino acid cysteine in absence (red) and presence of MB (blue) showing the displacement of $\mathrm{H}_{\alpha}$ and $\mathrm{H}_{\beta}$ chemical shifts further downfield upon oxidation together with their recovery in presence of deuterated DTT (green). c) Superposition of $2 \mathrm{D}{ }^{1} \mathrm{H}^{13}{ }^{13} \mathrm{C}$ NMR spectra of the amino acid cysteine in the absence (red) and presence of MB (blue) showing the $\left(\mathrm{C}_{\alpha}, \mathrm{H}_{\alpha}\right)$ and $\left(\mathrm{C}_{\beta}, \mathrm{H}_{\beta}\right)$ chemical shifts in their reduced and oxidized forms. 


\section{Mechanistic Basis of Phenothiazine-driven Inhibition of Tau Aggregation}

a)

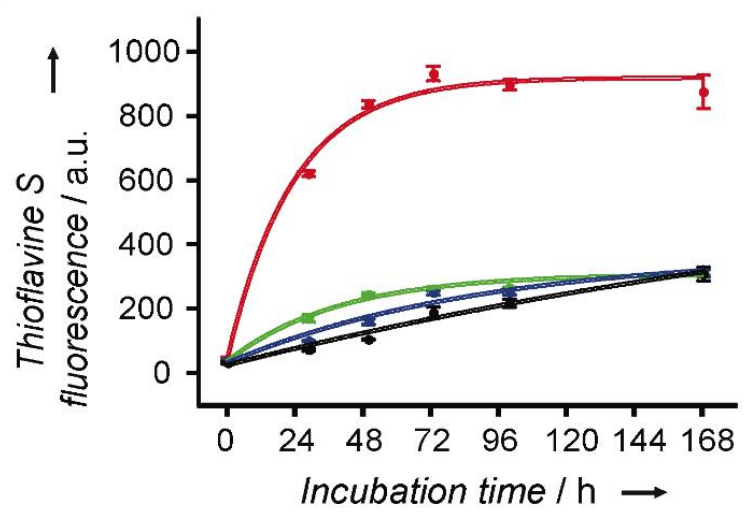

K19

no DTT

$0.5 \mathrm{mM}$ DTT

$1.0 \mathrm{mM}$ DTT

$5.0 \mathrm{mM}$ DTT

b)

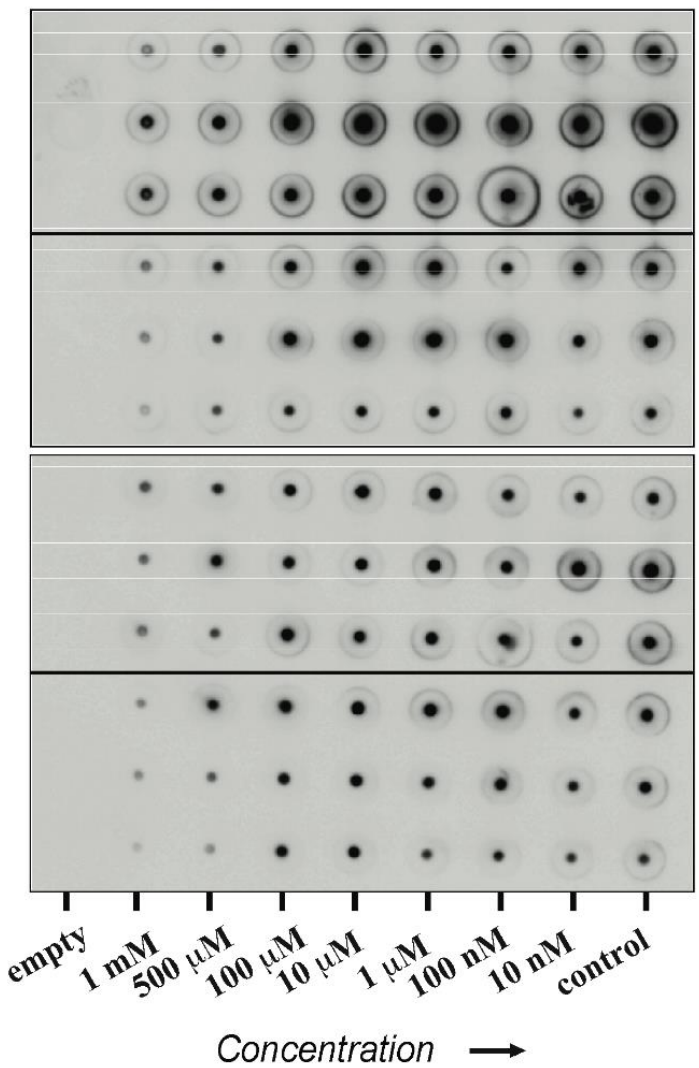

$0.5 \mathrm{mM}$ DTT

$1.0 \mathrm{mM}$ DTT

$5.0 \mathrm{mM}$ DTT

Figure S5. MB-driven inhibition of Tau aggregation under reducing conditions. a) Aggregation of the threerepeat Tau construct K19 under reducing conditions in the presence of increasing DTT concentrations but without MB as monitored by a Thioflavin S assay. b) Aggregation of K19 in the presence of different DTT and $\mathrm{MB}$ concentrations measured by the filter assay (see also Figure $2 \mathrm{~h}$ and Methods in Supporting Information for further details). 
3. Mechanistic Basis of Phenothiazine-driven Inhibition of Tau Aggregation

\section{SUPPORTING REFERENCES}

[1] O. Schweers, E. M. Mandelkow, J. Biernat, E. Mandelkow, Proc Natl Acad Sci U $S$ A 1995, 92, 8463-8467.

[2] M. D. Mukrasch, J. Biernat, M. von Bergen, C. Griesinger, E. Mandelkow, M. Zweckstetter, J Biol Chem 2005, 280, 24978-24986.

[3] D. H. Wu, A. D. Chen, C. S. Johnson, J Magn Reson Ser A 1995, 115, 260-264.

[4] P. V. Konarev, M. V. Petoukhov, V. V. Volkov, D. I. Svergun, J Appl Cryst 2006, $39,277-286$. 


\section{Imbalance of Hsp70 Family Variants Fosters Tau}

\section{Accumulation}

Umesh K. Jinwal ${ }^{1}$, Elias Akoury ${ }^{4}$, Jose F. Abisambra² ${ }^{2}$ John C. O'Leary III ${ }^{2}$, Andrea D. Thompson ${ }^{3}$, Laura J. Blair², Ying Jin², Justin Bacon², Bryce A. Nordhues ${ }^{2}$, Matthew Cockman ${ }^{2}$, Juan Zhang ${ }^{1}$, Pengfei Li $^{2}$, Bo Zhang ${ }^{2}$, Sergiy Borysov ${ }^{2}$, Vladimir N. Uversky ${ }^{2,5}$, Jacek Biernat ${ }^{6,7}$ Eckhard Mandelkow ${ }^{6,7}$, Jason E. Gestwicki $^{3}$, Markus Zweckstetter ${ }^{4,8}$, and Chad A. Dickey ${ }^{2}$

${ }^{1}$ Department of Pharmaceutical Sciences, and ${ }^{2}$ Department of Molecular Medicine, USF Health Byrd Alzheimer's Institute, University of South Florida; Tampa, FL, 33613; USA

${ }^{3}$ Department of Pathology; University of Michigan; Ann Arbor, MI, 48109; USA

${ }^{4}$ Department for NMR-based Structural Biology, Max Planck Institute for Biophysical Chemistry, Am Fassberg 11, 37077; Göttingen, Germany; ${ }^{5}$ Institute for Biological Instrumentation, Russian Academy of Sciences, 142290; Pushchino, Moscow Region, Russia; ${ }^{6}$ Max Planck Unit for Structural Molecular Biology at DESY, Notkestraße 85, 22607; Hamburg, Germany

${ }^{7}$ DZNE, German Center for Neurodegenerative Diseases, c/o CAESAR, Ludwig-Erhard-Allee 2, 53175; Bonn, Germany; ${ }^{8}$ German Center for Neurodegenerative Diseases (DZNE), Grisebachstr 5, 37077; Göttingen, Germany

* E. Akoury performed and analyzed NMR experiments and contributed to the manuscript. 


\section{Imbalance of Hsp70 Family Variants Fosters Tau Accumulation}

\section{ABSTRACT}

Dysfunctional tau accumulation is a major contributing factor in tauopathies, and the heat shock protein 70 (Hsp70) seems to play an important role in this accumulation. Several reports suggest that Hsp70 proteins can cause tau degradation to be accelerated or slowed, but how these opposing activities are controlled is unclear. Here we demonstrate that highly homologous variants in the Hsp70 family can have opposing effects on tau clearance kinetics. When over-expressed in a tetracycline-based protein chase model, constitutive Hsc70 and inducible Hsp72 slowed or accelerated tau clearance, respectively. Tau synergized with Hsc70, but not Hsp72, to promote microtubule assembly at nearly twice the rate of either Hsp70 homolog in reconstituted, ATP-regenerating Xenopus extracts supplemented with rhodamine-labeled tubulin and human recombinant Hsp72 and Hsc70. Nuclear magnetic resonance spectroscopy with human recombinant protein revealed that Hsp72 had greater affinity for tau than $\mathrm{Hsc} 70\left(\mathrm{I} / \mathrm{I}_{0}\right.$ ratio difference of 0.3$)$, but Hsc 70 was thirty times more abundant than Hsp72 in human and mouse brain tissue. This indicates that the predominant Hsp70 variant in the brain is Hsc70, suggesting that the brain environment primarily supports slower tau clearance. Despite its capacity to clear tau, Hsp72 was not induced in the Alzheimer's brain, suggesting a mechanism for ageassociated onset of the disease. Through the use of chimeras that blended the domains of Hsp72 and Hsc70, we determined that the reason for these differences between Hsc70 and Hsp72 with regard to tau clearance kinetics lies within their C-terminal domains, which are essential for their interactions with substrates and co-chaperones. Hsp72 but not Hsc70 in the presence of tau was able to recruit the co-chaperone ubiquitin ligase CHIP, which is known to facilitate the ubiquitination of tau, describing a possible mechanism of how the C-termini of these homologous Hsp70 variants can differentially regulate tau triage. Thus, 


\section{Imbalance of Hsp70 Family Variants Fosters Tau Accumulation}

efforts to promote Hsp72 expression and inhibit Hsc70 could be therapeutically relevant for tauopathies.

\section{INTRODUCTION}

The microtubule-associated protein tau, when disengaged from microtubules, becomes deleterious in tauopathies (1-4). Part of this pathogenesis involves structural changes that promote its aggregation. Chaperone machinery, and in particular members of the heat shock protein 70 (Hsp70) family, are engaged with tau during these pathogenic events, and these proteins dramatically affect tau stability and function at this critical juncture (5-7). In mammalian systems, there are approximately 13 homologous Hsp70 genes, including the primary cytosolic variants Hsc70 (HSPA8) and Hsp72 (HSPA1A). Though these two variants share nearly $80 \%$ sequence identity, some functional differences have been reported $(8,9)$. Hsc70 is constitutively expressed (10), while Hsp72 is expressed in response to stress (11). Moreover, the C-termini of these chaperones, known to interact with key co-chaperone effectors containing a tetratricopeptide (TPR) repeat motif, is where their sequences differ the most (12). Despite these observations, the term Hsp70 is often used to generically refer to both Hsc70 and Hsp72.

We and others have shown that the collective Hsp70 chaperone network, and in particular Hsp72 and Hsc70, influences tau conformation, degradation, and aggregation kinetics. But how these fates are chosen for tau have been challenging to define. For example, some studies strongly suggest that over-expression of an unspecified Hsp70 variant can facilitate tau clearance (5), whereas others suggest that both Hsp72 and Hsc70 restore tau's binding to microtubules $(1,7)$. Though differences in the cellular environment, such as stress (12) and client abundance (13) may contribute to this discrepancy, it has yet 


\section{Imbalance of Hsp70 Family Variants Fosters Tau Accumulation}

to be definitely shown how these different fates for tau are chosen by the Hsp70 machinery. We show here that tau fate is critically linked to both Hsc70 and Hsp72.

\section{MATERIALS AND METHODS}

Materials. Hsc70 and Hsp72 antibodies were purchased from Stressgen, Famingdale, NY, USA and specificity was validated as previously described (8). Total tau H150 antibody was purchased from Santa Cruz Biotechnology, Santa Cruz, CA, USA. Actin - and GAPDH antibodies were from Sigma, St. Louis, MO, USA and Biodesign International, Memphis, TN, USA, respectively. Myc and Flag antibodies were from Roche, Indianapolis, IN, USA and Sigma, respectively. CHIP antibody was provided by Cell Signaling, Danvers, MA, USA. Phosphatase inhibitor cocktails (1 \& 3), albendazole and celastrol were purchased from Sigma. Cell lysis buffer- M-PER was purchased from Fisher Scientific, Pittsburgh, PA, USA. Secondary antibodies horse-radish peroxidase linked were purchased from Southern Biotech Inc., Birmingham, AL, USA. Lipofectamine 2000 and secondary antibodies conjugated to flourophore were purchased from Invitrogen, Grand Island, NY, USA. Plasmids for wild type human Hsc70, Hsp72 and chimera were generated in the pCMV6 vector (Origene, Rockville, MD, USA) which was then engineered to contain an N-terminal Flag tag. Mutant constructs were generated using the Site directed mutagenesis kit from Stratagene, Santa Clara, CA, USA. All other clones were in pCDNA3.1 plasmid. Human brain tissue was provided from the University of California at Irvine MIND.

Cell Culture, Immunoblotting, Dot blot, immunostaining and Immunoprecipitation. Human neuroblastoma IMR-32, HEK and HeLa cells were maintained in the OptiMEM media supplemented with $10 \%$ heat inactivated FBS and 1\% 


\section{Imbalance of Hsp70 Family Variants Fosters Tau Accumulation}

penicillin/streptomycin antibiotic solution. All the transfections, immunoblotting and immunoprecipitation experiments were performed as described previously (14). Dot blots were performed using the Whatman Minifold I apparatus on nitrocellulose (Whatman, Piscataway, NJ, USA). All values were normalized to Ponceau S. Recombinant Hsp72 and Hsc70 were applied at indicated molar concentrations in PBS. Lysates from human AD brain tissue and control brain tissue were spotted at $20 \mu \mathrm{g}$ total protein. Quantification of Western blot and dot blot data was performed using replicate values from ScionImage or Image $\mathrm{J}$ densitometric software suites. Dot blot calculations were performed using a semilog linear regression analysis. Immunostaining of mouse brain tissue was performed as previously described $(15,16)$.

Tetracycline-Based Protein Chase HEK Cell Model Inducible HEK cell line was generated by the insertion of human wild type Tau 4R0N DNA into a pCDNA 4/TO plasmid vector carrying Zeocin selection (Invitrogen). The Tau/TO plasmid construct was transfected into the Tetracycline-Regulated Expression (T-REx ${ }^{\mathrm{TM}}$; Invitrogen) HEK cell lines stably expressing tetracycline (Tet) repressor under the selection of Blasticidin. After 24 hours of DNA transfection, Zeocin $(400 \mu \mathrm{g} / \mathrm{ml})$ and Blasticidin $(5 \mu \mathrm{g} / \mathrm{ml})$ were added to the media (DMEM containing 10\%FBS, 2mM L-glutamine, 1\% Pen-Strep). Every 3-4 days later media was replaced with fresh media containing Zeocin and Blastidin. After 3 weeks, double stable cell colonies were picked-up and transferred into 6 well plates. These colonies were induced by tetracycline $(1 \mu \mathrm{g} / \mathrm{ml})$ and tested for tau expression by western blot. Tau expressing double stable HEK cells were maintained under the selection of Zeocin and Blasticidin and used for the experimentation. Following transfection with Hsp72 or Hsc70 clones, tetracycline-containing media was removed and replaced with normal media. Tau levels were reduced over time to near basal levels. 


\section{Imbalance of Hsp70 Family Variants Fosters Tau Accumulation}

Live Cell Imaging. HEK-293 cells were plated onto a 6-well plate and maintained in DMEM plus $10 \%$ fetal bovine serum and $1 \%$ of $200 \mathrm{mM}$ L-glutamine. Plasmid transfections were done utilizing Lipofectamine 2000 (Invitrogen). HEK 293 cells were transfected with $2 \mu \mathrm{g}$ of Hsc 70 DNA, $1 \mu \mathrm{g}$ of Tubulin-GFP DNA and $1 \mu \mathrm{g}$ of Tau-RFP DNA. The cells were incubated overnight with the Lipofectamine/plasmid mixture in Opti-MEM medium, and replaced with fresh complete media for an additional 36h. An Olympus FV1000 MPE multiphoton laser scanning microscope was used to capture images. Image analysis was completed with the Olympus FV10-ASW software, and significance was assessed by Pearson's correlation coefficient. Cells were then lysed and analyzed by Western blot to confirm over-expression of Hsp72 and Hsc70.

Recombinant Protein Production. The coding regions for WT 4R0N Tau, Hsp72, and Hsc70 were separately PCR amplified and subcloned into pET28A vectors with Nterminal His6-tags. Resulting plasmids were transformed into E. coli OneShot BL21 Star (DE3) cells (Invitrogen) and 10mL starter cultures were grown overnight in Luria Bertani (LB) media with $30 \mu \mathrm{g} / \mathrm{ml}$ kanamycin. Cells were then inoculated into 1.0L LB media, grown at $37^{\circ} \mathrm{C}$ to $\mathrm{A}_{600} \sim 0.7-0.8$, and induced with $1 \mathrm{mM}$ isopropyl $\beta-\mathrm{D}-1$ thiogalactopyranoside (IPTG). After 3 hours of continued growth at $37^{\circ} \mathrm{C}$, cells were harvested by centrifugation and resuspended in lysis buffer $(500 \mathrm{mM} \mathrm{NaCl}, 20 \mathrm{mM}$ Tris$\mathrm{HCl}$, 5mM imidazole, 1x protease inhibitor cocktail III (Calbiochem, Billerica, MA, USA), $1 \mathrm{mM}$ PMSF, $\mathrm{pH} 8.0)$. The cells were then lysed by sonication, centrifuged (18200g, $30 \mathrm{~min}, 4^{\circ} \mathrm{C}$ ), and supernatant was loaded onto $5 \mathrm{~mL}$ of charged Ni-NTA agarose beads (Qiagen, Valencia, CA, USA). Protein was eluted in lysis buffer containing $250 \mathrm{mM}$ imidazole. Eluent was concentrated to $2 \mathrm{~mL}$ and loaded onto a HiLoad 16/60 Superdex 200 


\section{Imbalance of Hsp70 Family Variants Fosters Tau Accumulation}

pg size exclusion column. Purified fractions were dialyzed in 2 steps into $10 \mathrm{mM}$ phosphate buffer $(\mathrm{pH} 7.5)$ and concentrations were determined by BCA.

In vitro microtubule assembly assay. These assays were performed as previously described $(1,14)$. Briefly, S-phase Xenopus egg extracts were prepared according to a standard protocol ${ }^{(17)}$ and supplemented with 5\% DMSO to stimulate microtubule polymerization as described ${ }^{(18)}$. Purified recombinant wt tau, Hsp72, and Hsc70 proteins were added at the final concentrations of 40,80 , and $80 \mathrm{ng} / \mu \mathrm{l}$. The extracts were incubated $30 \mathrm{~min}$ at room temperature and obtained microtubule structures were visualized by fluorescence of incorporated Rhodamine-labelled tubulin.

NMR. ${ }^{15} \mathrm{~N}$ labeled human Tau protein was expressed and purified as described previously (19). NMR experiments were recorded at $5{ }^{0} \mathrm{C}$ on a Bruker Avance $800 \mathrm{MHz}$ spectrometer equipped with a cryogenic probe. NMR samples contained $50 \mu \mathrm{M}^{15} \mathrm{~N}$ single labeled Tau protein in $50 \mathrm{mM}$ phosphate buffer and $10 \mathrm{mM} \mathrm{NaCl}, \mathrm{pH} 6.8,1 \mathrm{mM}$ DTT and $10 \%(\mathrm{v} / \mathrm{v}) \mathrm{D}_{2} \mathrm{O}$. Unlabeled Hsp72 and Hsc70 samples were prepared in the same buffer.

Two-dimensional ${ }^{1} \mathrm{H}-{ }^{15} \mathrm{~N}$ heteronuclear single quantum coherence (HSQC) spectra were acquired using 600 complex points and 128 scans per increment with spectral widths of $8012 \mathrm{~Hz}$ and $1944 \mathrm{~Hz}$ in the ${ }^{1} \mathrm{H}$ and ${ }^{15} \mathrm{~N}$ dimensions, respectively. Spectra were processed with NMRPipe (20) and analyzed using CcpNMR Analysis(21). NMR intensity ratio plots were reported with a 3-residues averaging window.

Immunostaining Mouse brains were processed for immunohistochemical analysis as described previously $(16,22)$. Briefly, mice brain sections were immersed in $3 \% \mathrm{H}_{2} \mathrm{O}_{2}$ solution for 15 min at RT. After washing with PBS sections, were permeabilized by immersing in the $4 \%$ donkey serum blocking buffer (supplemented with $1.83 \%$ lysine, and 2\% Triton X-100) for 30 min at RT. Sections were treated with Anti-HSC70 (1:20000) and 


\section{Imbalance of Hsp70 Family Variants Fosters Tau Accumulation}

anti-Hsp72 (1:300) primary antibodies for overnight at $4^{\circ} \mathrm{C}$. After washing with PBS, sections were incubated with biotinylated anti-rat (1:1000) and anti-mouse (1:3000) secondary antibodies (southern biotech) for two hours and then with streptavidinperoxidase. The peroxidase reactions consisted of $1.4 \mathrm{mM}$ diaminobenzidine with $0.03 \%$ hydrogen peroxide in PBS for 5 min. Finally, stained sections were mounted on glass slides, dehydrated, cleaned using histoclean and cover slipped. Imaging was performed with the Zeiss Mirax slide scanning microscope.

Fluorescent polarization (FP) assay FP assays were carried out essentially as previously described (23). The peptide FITC-HLA (RENLRIARLY) was synthesized on Wang resin using microwave-assisted DIC/HOBt solid-phase peptide synthesis. It was capped with two $\beta$-alanine residues and labeled on resin via the $\mathrm{N}$-terminus with fluorescein-5-isothiocyanate (Anaspec). The final product was obtained in $>90 \%$ purity. FP assays were carried out in $384-w e l l$ plates $(20 \mu \mathrm{L}$ final volume) with a 30 minute incubation. Conditions: Hsp72 $(2 \mu \mathrm{M})$ or Hsc70 $(4 \mu \mathrm{M}), 25$ nM FITC-HLA probe, 100 $\mathrm{mM}$ Tris buffer, $20 \mathrm{mM} \mathrm{KCl,} 6 \mathrm{mM} \mathrm{MgCl2,} \mathrm{pH} \mathrm{7.4.}$

Sequence Alignment. Global sequence alignment of H. sapiens Hsp72 (HspA1A/ A29160) and Hsc70 (HspA8/A27077) using PRALINE (http://www.ibi.vu.nl/programs/ pralinewww/) with manual adjustment. Figure is drawn to scale and the degree of residue conservancy is illustrated by color. 


\section{Imbalance of Hsp70 Family Variants Fosters Tau Accumulation}

\section{RESULTS}

Hsp70 proteins have the capacity to both reduce and preserve tau in cells in addition to regulating its association with microtubules $(1,5,7)$. The reasons for these functional differences are unclear. We speculated that one possible reason could be the unique effects of distinct Hsp70 variants. Since tau is a cytosolic protein, we investigated the relationship of tau with the two major cytosolic Hsp70 variants: Hsp72, which is induced by stress (11), and Hsc70, which is constitutively expressed (10). The effects of Hsp72 and Hsc70 overexpression on tau stability were initially tested using a tetracycline-based protein chase model in HEK cells (TauTO/6TR). As shown in Figure 1A, tau levels are undetectable prior to tetracycline treatment and maximally induced 72 hours after tetracycline treatment. Once the tetracycline is removed, tau levels are depleted by natural turnover processes over the course of 72 hours (Fig 1B). Using this model, we demonstrated that Hsc70 slowed while Hsp72 accelerated tau clearance (Figs. 1C \& D). This indicated that these two highly similar proteins were distinctly affecting tau turnover. We then confirmed that endogenous tau could interact with endogenous Hsc70 and Hsp72 using co-immunoprecipitation assays in neuronal IMR-32 cell lysates analyzed by immunoblot. The neuronal cells were first treated for 24 hours with celastrol, a compound that is known to enhance the expression of heat shock proteins including Hsp72 (24) and destabilize microtubules (25). This was done to determine whether inducing Hsp72 expression might facilitate the interaction of Hsp72 with tau. Then, 30 minutes prior to harvest, these cells were also treated with the microtubule destabilizer albendazole to release tau from microtubules, a process that was previously found to enhance the tau/Hsc70 interaction (1). As previously reported, Hsc70 bound more tau when microtubules were destabilized, but celastrol treatment had no impact on Hsc70 binding to tau (Figs 1E). Conversely, for endogenous Hsp72 to bind tau in a 


\section{Imbalance of Hsp70 Family Variants Fosters Tau Accumulation}

similar manner, Hsp72 levels had to be induced by celastrol treatment for it to bind tau in a manner similar to Hsc70 (Figs. 1E). These data suggested that relative levels of these two proteins could play a critical part in deciding whether tau was to be preserved or degraded. Our data suggested that Hsp72 was accelerating tau clearance while Hsc70 was slowing it. Therefore, we speculated that increasing the levels of Hsp72 could overcome any preservative effects of Hsc70 on tau. Cells over-expressing tau were transfected with equivalent amounts of Hsc70 along with increasing amounts of Hsp72. Analysis of these lysates by immunoblot revealed that the enhanced steady-state levels of tau by Hsc70 could indeed be negated by Hsp72 over-expression (Figs 1F \& G). 


\section{Imbalance of Hsp70 Family Variants Fosters Tau Accumulation}

A.

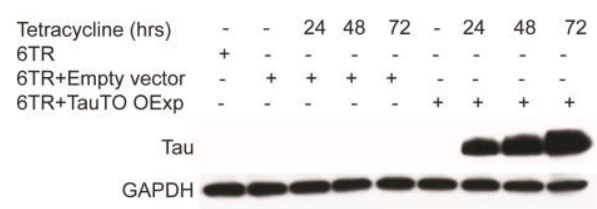

B.

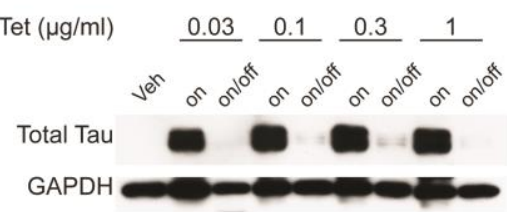

E.

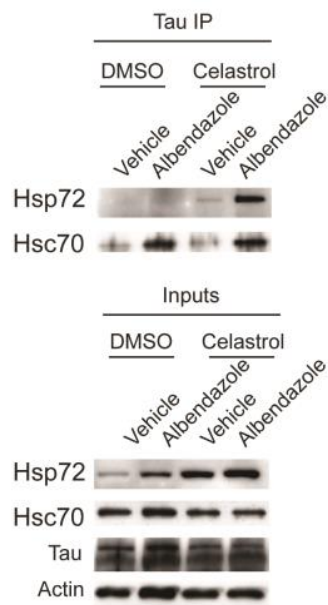

C.

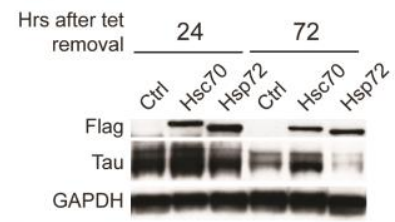

D.

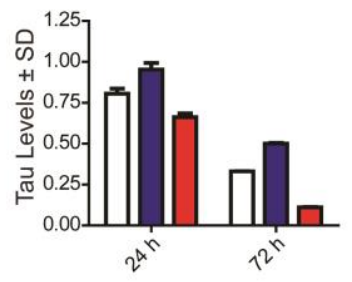

F.

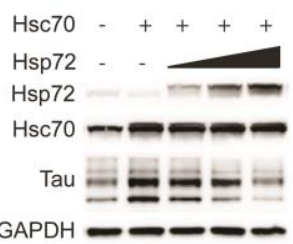

G.

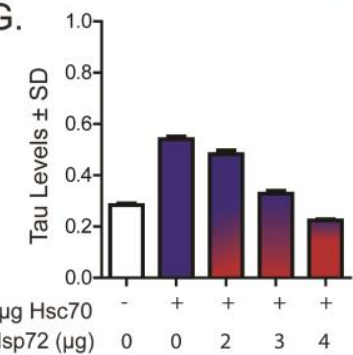

Figure 1: Hsp72 and Hsc70 have opposing effects on tau.

(A) Western blot of lysates from HEK cells transiently transfected with tau driven by the minimal CMV promoter controlled by the tetracycline response element (TauTO) or empty vector, and transiently transfected with the tetracycline repressor protein (6TR). Cells were then treated with tetracycline and harvested at indicated time points. (B) HEK cells stably transfected with TauTO and the 6TR were treated with indicated concentration of tetracycline (Tet) for 24 hours. After 24 hours, Tet containing media was replaced with media either containing Tet (on) or without Tet (on/off) and these cells were incubated for an additional 72 hours (96 hours total). Lysates were analyzed by Western blot. (C) TauTO/6TR stably transfected cells were treated with tetracycline for $24 \mathrm{~h}$. Cells were then transfected with Hsc70 or Hsp72 for $24 \mathrm{~h}$ and tetracycline was removed. Cells were harvested at indicated times following tetracycline removal. (B) Quantification of tau levels in C shown as a percentage of empty vector transfected cells (Ctrl) \pm standard deviation (SD) after GAPDH normalization $(\mathrm{n}=3)$. Open bars, blue bars and red bars indicate vector, Hsc70and Hsp72-transfected cells, respectively. (C) Co-immunoprecipitation of tau from IMR-32 cell lysates treated with DMSO alone and albendazole with DMSO, or celastrol alone and albendazole with celastrol. 3 $\mu \mathrm{M}$ Celastrol was applied 24 hours, followed by $20 \mu \mathrm{M}$ Albendazole treatment for 30 minutes. Levels of Hsp72 and Hsc70 bound to tau were assessed. Inputs indicates whole lysates. (F) Western blot of tau in lysates of HEK cells co-transfected with Hsc70 and increasing amounts of Hsp72. (G) Quantification of tau levels in $\mathrm{F}$ shown as a percentage of Vector transfected cells \pm standard deviation (SD) after GAPDH normalization. 


\section{Imbalance of Hsp70 Family Variants Fosters Tau Accumulation}

Previous work suggested that Hsp70 proteins could promote the association of tau with microtubules (7); however and based on our results, we speculated that Hsc70 would affect this more potently than Hsp72. Using co-localization analyses of GFP-tagged tubulin and RFP-tagged tau in live cells, we found a slight increase in tau-tubulin associations induced by Hsc70 (Figs. 2A-C). However, Hsp72 over-expression significantly decreased the association of tau with tubulin, further suggesting that Hsp72 promoted tau degradation (Figs. 2A-C). Western blot confirmed over-expression of both Hsp72 and Hsc70 (Fig 2D). While these studies supported opposing roles for these two Hsp70 variants on tau function, it still was not clear whether Hsp72 and Hsc70 had direct effects on the ability of tau to regulate microtubule formation. To better evaluate this, we utilized microscopy of in vitro cell-free Xenopus egg extracts supplemented with fluorescently-labeled tubulin and combinations of either recombinant $4 \mathrm{R} 0 \mathrm{~N}$ tau, Hsp72, or Hsc70 alone; or 4R0N tau combined with Hsp72 or Hsc70. Hsc70, Hsp72, and tau were all able to promote microtubule assembly alone relative to control, but Hsc70 and tau together appeared to dramatically synergize this process, producing densely-packed microtubule filaments (Figs 3A \& B). Conversely, Hsp72 did not appear to synergize with tau: In fact, there was a slight reduction in microtubule network formation when Hsp72 alone was added with tau.

These findings suggested that despite their sequence similarity, Hsp72 and Hsc70 caused distinct and even opposing effects on tau's function and stability. To determine whether this was caused by differing binding locations on tau, nuclear magnetic resonance (NMR) spectroscopy was used to identify tau residues essential for Hsp72 and Hsc70 interactions. Changes in NMR signal position and intensity in two-dimensional ${ }^{1} \mathrm{H}-{ }^{15} \mathrm{~N}$ heteronuclear single quantum coherence (HSQC) spectra of the 441-residue full length tau protein were observed for both Hsp70 variants in a concentration-dependent manner (Fig 


\section{Imbalance of Hsp70 Family Variants Fosters Tau Accumulation}

4A). The observed changes were caused by an exchange of tau between the free and the Hsp72/Hsc70-bound state. Strong signal broadening was particularly evident in the second and third repeats of tau comprising the two hexapeptides ${ }^{275} \mathrm{VQIINK}^{280}$ and ${ }^{306} \mathrm{VQIVYK}^{311}$ essential for aggregation of tau into paired helical filaments (Fig 4B \& C). This data was consistent with previous studies belying the importance of these motifs in chaperone mediated autophagy (26) and interaction with Hsc70 proteins (27). Strikingly, much more pronounced changes in repeats 2 and 3 were observed for Hsp72 than Hsc70 (Fig 4B), indicating differences in the interaction between Hsp72 and Hsc70 with Tau (Fig 4C). In

addition, weaker interactions were observed with the residue stretches ${ }^{346} \mathrm{FKDRVQSK}^{353}$ and ${ }^{375} \mathrm{KLTFRE}^{380}$. 


\section{Imbalance of Hsp70 Family Variants Fosters Tau Accumulation}

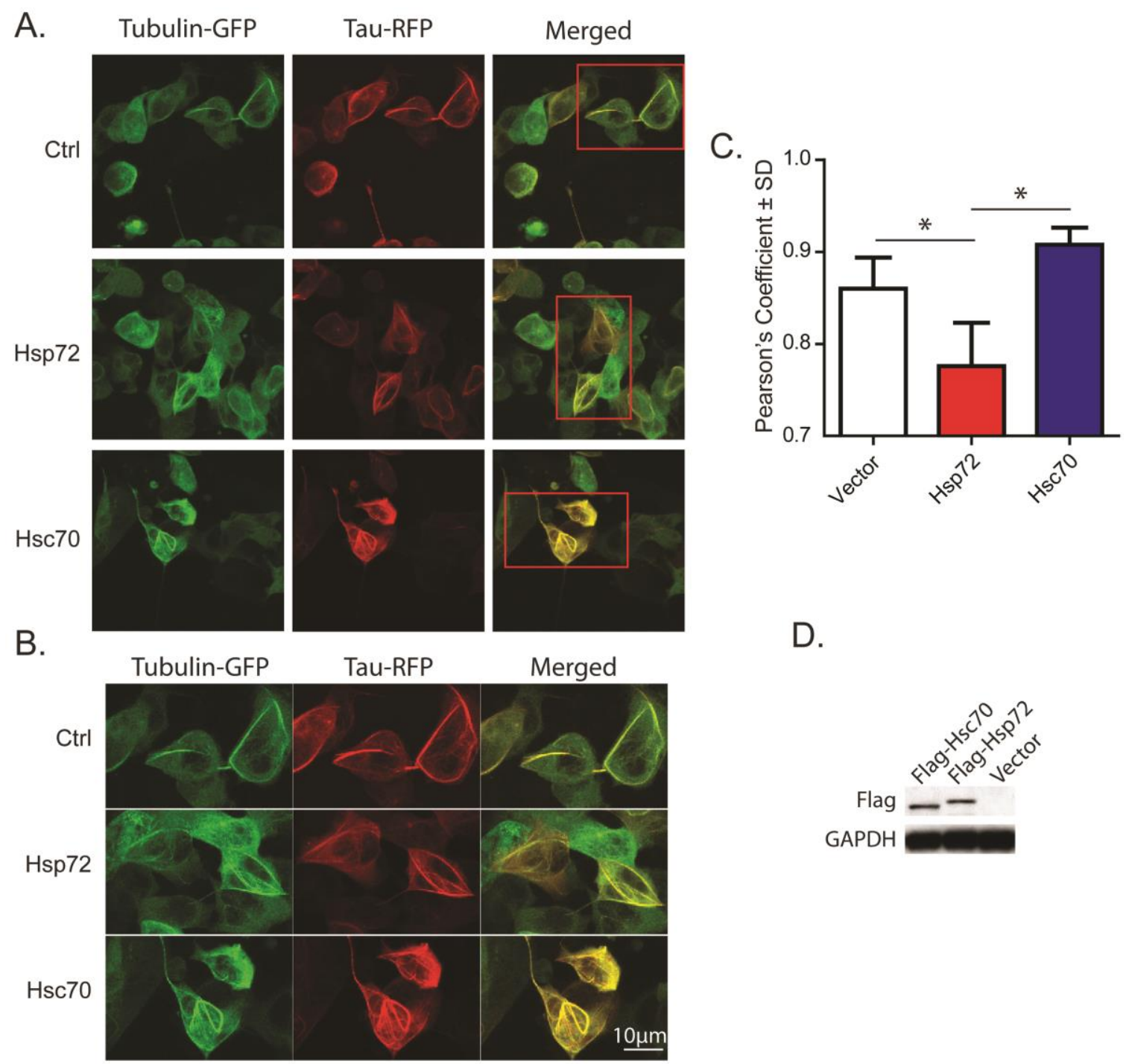

Figure 2: Hsc70, but not Hsp72, enhances the co-localization of tau with tubulin in cells.

(A) Representative two-photon fluorescent images of live HEK cells co-transfected with tubulin-GFP and tau-RFP fusion constructs. Cells transfected with vector (Ctrl) or Hsc70 showed significant co-localization between tau and tubulin as compared to cells transfected with Hsp72. (B) Higher magnification imaging of areas boxed in red in panel A. (C) Graph demonstrating the Pearson's co-localization coefficient derived from 12 different images \pm standard deviation (SD). * indicates $p<0.05$. (D) Western blot of cell lysates confirming over-expression of Flag-tagged Hsp72 and Hsc70. 


\section{Imbalance of Hsp70 Family Variants Fosters Tau Accumulation}

A.
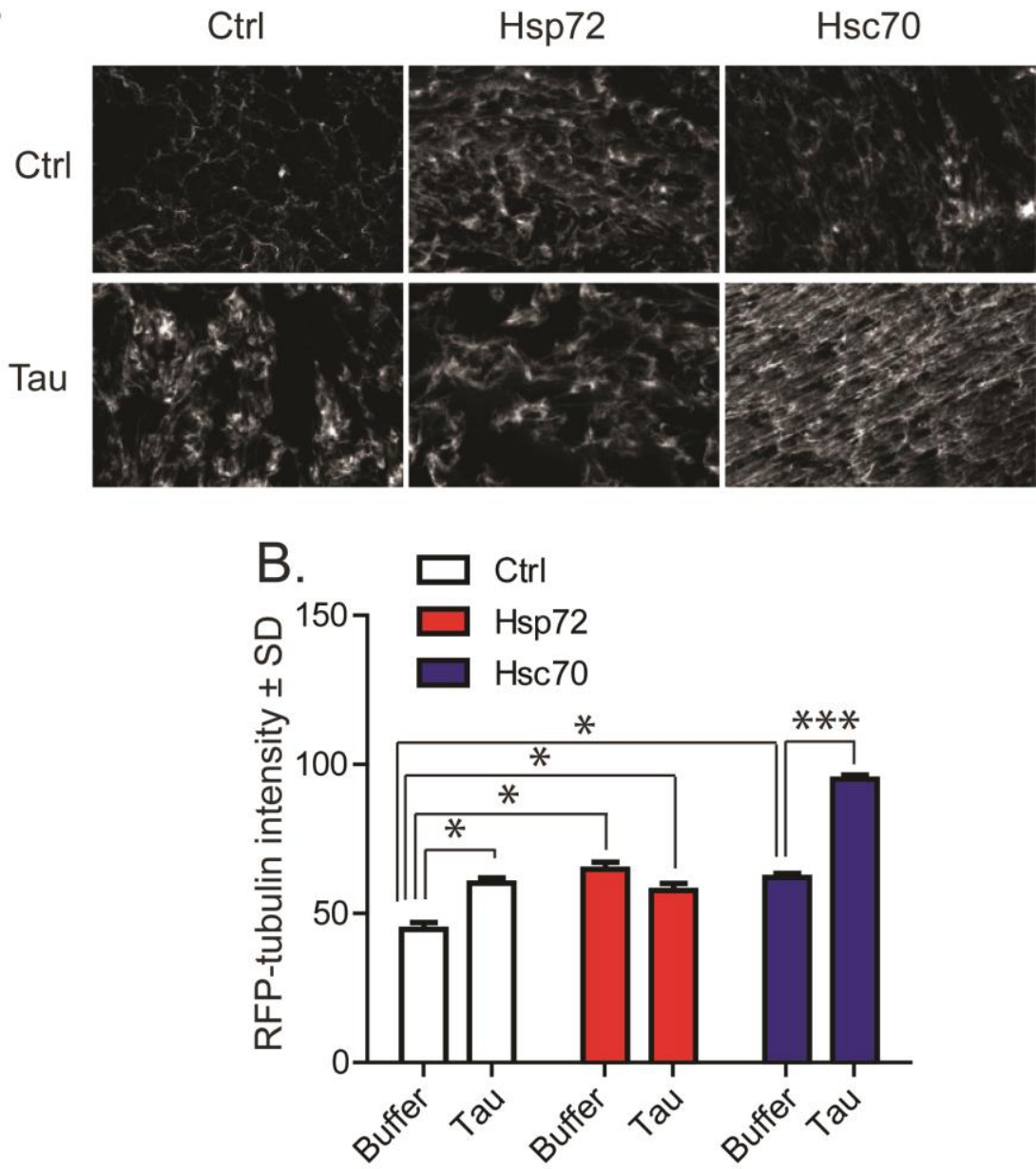

Figure 3: Hsc70, but not Hsp72, synergizes with tau to promote microtubule assembly.

(A) Representative fluorescent images of Xenopus extracts supplemented with rhodamine-labelled tubulin and indicated combinations of recombinant tau, Hsp72 and Hsc70 (n=10). (B) Graph demonstrating that tau, Hsp72 and Hsc70 alone could promote microtubule assembly, but only Hsc70 combined with tau to dramatically influence microtubule formation. * indicates $p<0.05$, *** indicates $p<0.001$. 


\section{Imbalance of Hsp70 Family Variants Fosters Tau Accumulation}

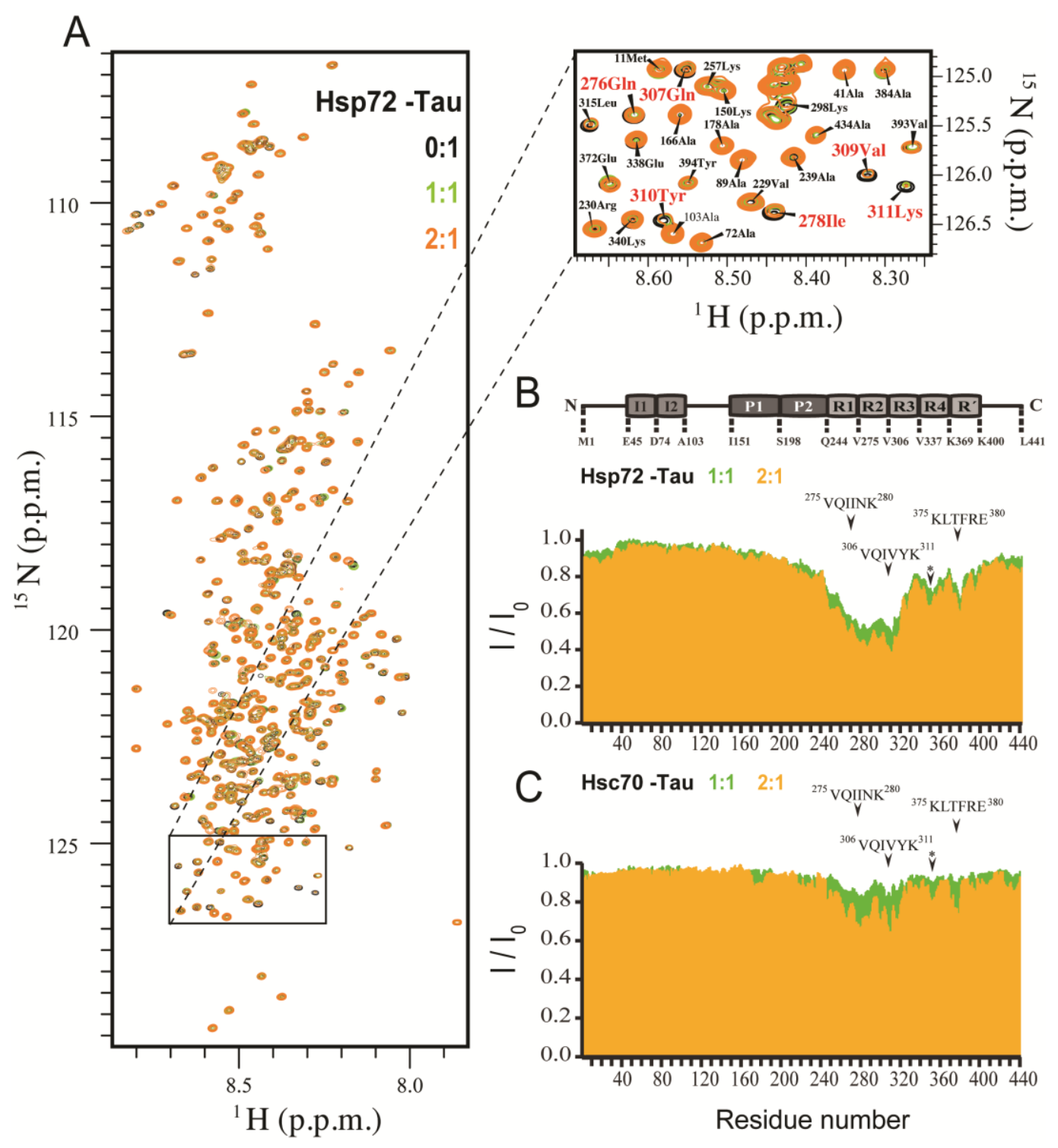

Figure 4: Hsp72 binds Tau with higher affinity than Hsc70.

(A) Superposition of $2 \mathrm{D}{ }^{1} \mathrm{H}-{ }^{15} \mathrm{~N}$ HSQC spectra of Tau protein $(50 \mu \mathrm{M})$ in the absence (black) and presence of an equimolar (green) and 2-fold excess ratios of Hsp72 (orange). The inset highlights the resonances of the two hexapeptides and their attenuation in a concentration dependent manner. (B and C) NMR intensity ratios $\mathrm{I} / \mathrm{I}_{0}\left(\mathrm{I}=\right.$ intensity of Tau resonances in presence of Hsp72 or Hsc70; $\mathrm{I}_{0}=$ peak intensities of free Tau) from HSQC spectra. Signal broadening $\left(\mathrm{I} / \mathrm{I}_{0}<1.0\right)$ is due to exchange of Tau between the free conformations and those in the presence of Hsp72 or Hsc70. NMR signal broadening of Tau at increasing concentrations of (B) Hsp72 and (C) Hsc70 (1:1 green, 2:1 orange). The domain organization of Tau is shown above (I = insert, $\mathrm{P}=$ proline-rich region, and $\mathrm{R}=$ pseudo-repeat). The asterisk corresponds to the residues stretch ${ }^{346}$ FKDRVQSK ${ }^{353}$. 


\section{Imbalance of Hsp70 Family Variants Fosters Tau Accumulation}

We sought to better understand the mechanism responsible for these distinct direct effects of Hsp72 and Hsc70 on tau, and why in particular Hsp72 could out-compete Hsc70 for tau binding. We performed fluorescence polarization assays in which recombinant Hsp72 and Hsc70 were incubated with both a labeled peptide known to interact with their substrate binding domains and increasing concentrations of recombinant tau. Tau was able to compete with the fluorescent mock-substrate peptide in a dose dependent manner, suggesting that tau was interacting with the substrate binding domains of Hsp72 and Hsc70 (Fig 5A). While these data showed where tau interacted with Hsp72 and Hsc70, it did not explain why these two proteins were causing differential effects on tau biology in cells. Using the Praline algorithms, we found that while these two proteins shared $>80 \%$ similarity in their complete protein sequence, their C-terminal regions had the highest sequence variation (30\%; Fig. 5B \& C). Therefore, chimeras were constructed to blend the nucleotide binding domains (NBD), substrate binding domains (SBD), and the most variable C-terminal domains (CTD) from Hsp72 and Hsc70 (Fig. 5D) to determine which region was most essential for the distinct tau effects. Over-expression of these chimeras in cells revealed that constructs possessing the CTD of Hsc70 preserved tau, while those containing the CTD of Hsp72 facilitated tau clearance (Fig. 5E \& F). The NBD and SBD of both proteins appeared to be interchangeable.

This C-terminal interaction suggested an important role for co-factors/cochaperones of Hsp72 and Hsc70 that bind to this same region in regulating tau stability. To test this, cells over-expressing tau and either Hsp72 or Hsc70 were transfected with siRNA targeting CHIP, a ubiquitin ligase co-chaperone known to regulate tau turnover $(5,6,28)$. Western blot of these lysates showed that knocking down CHIP preserved tau despite overexpression of Hsp72 or Hsc70 (Figs. 6A \& B). This suggested that CHIP expression was 


\section{Imbalance of Hsp70 Family Variants Fosters Tau Accumulation}

important for the effects of Hsp72 and Hsc70 on tau in cells. To assess whether CHIP binding to Hsp72 and Hsc70 was different, we performed co-immunoprecipitation using anti-Flag antibody in cells over-expressing myc-tagged CHIP, tau, and either Flag-tagged Hsc70 or Flag-tagged Hsp72. In cells over-expressing tau, the number of CHIP/Hsp72 complexes was significantly greater than the number of CHIP/Hsc70 complexes (Fig. 6C), but surprisingly, in cells not over-expressing tau, the binding of CHIP to Hsc70 and Hsp72 was equivalent (Fig. 6D). In this way, Hsp72, but not Hsc70, in the presence of tau and other co-factors in the cell would favor CHIP binding to tau. 


\section{Imbalance of Hsp70 Family Variants Fosters Tau Accumulation}

A.

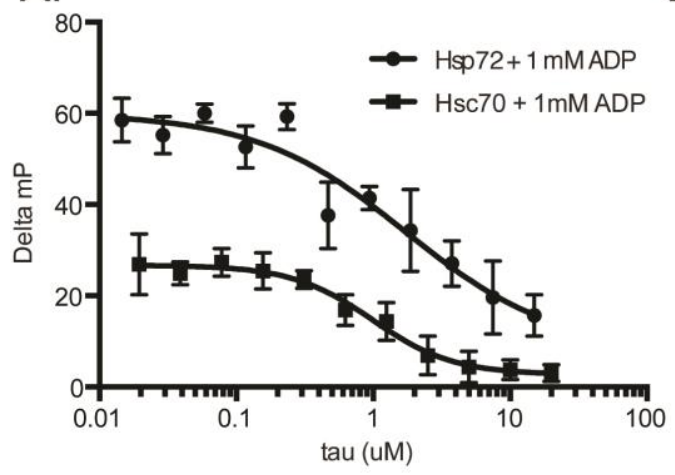

C.

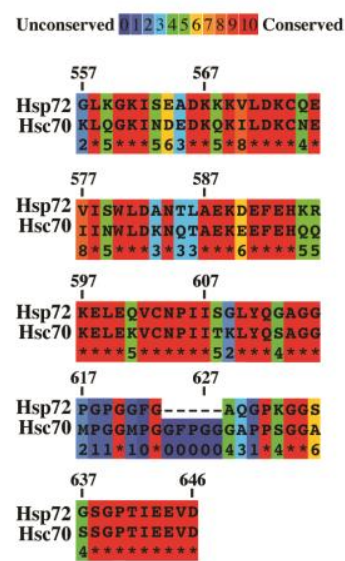

E.

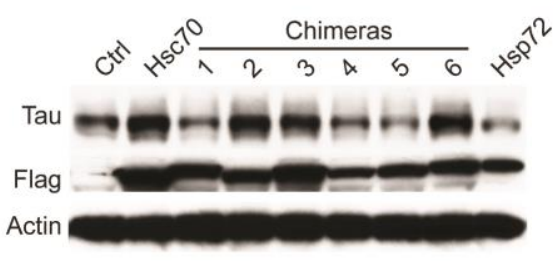

B.

Results color-coded for amino acid conservation; $92 \%$ conservation throughout

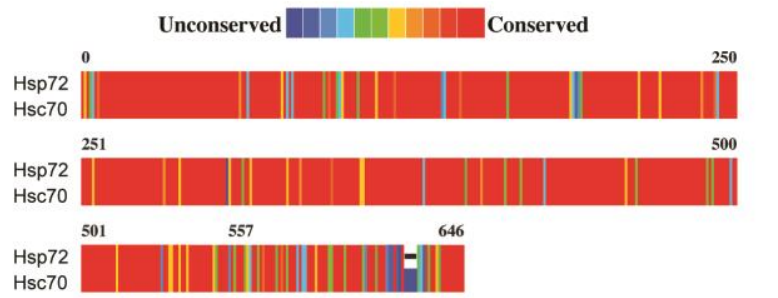

D.

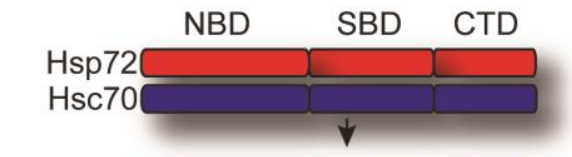

chimera 1

chimera 2

chimera 3

chimera 4

chimera 5

chimera 6

F.

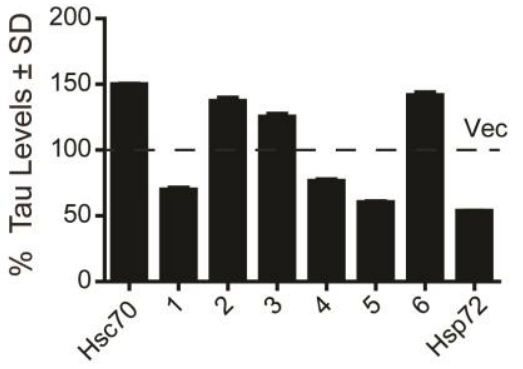

Figure 5: The C-terminal domains from Hsc70 and Hsp72 are essential for tau triage.

(A) Fluorescence polarization (FP) assays with recombinant Hsp72 or Hsc70 in the presence of 1mM ADP, the FITC-HLA (RENLRIARLY) peptide and increasing amounts of recombinant tau were carried out in 384well plates $(20 \mu \mathrm{L}$ final volume) with a 30 minute incubation. Results are the average of experiments performed in triplicate.and error bars represent standard error of the mean. (B and C) Sequence alignment showing 29\% sequence variability in the C-termini of Hsp72 and Hsc70. (D) Schematic of Hsc70/Hsp72 chimera design. (E) Western blot of lysates from HeLa cells stably expressing tau and transfected with Flagtagged Hsc70/Hsp72 chimeras. (F) Quantification of tau levels in D shown as a percentage of Vector transfected cells $(\mathrm{Ctrl}) \pm$ standard deviation $(\mathrm{SD})$ after GAPDH normalization $(\mathrm{n}=3)$. 


\section{Imbalance of Hsp70 Family Variants Fosters Tau Accumulation}

A.

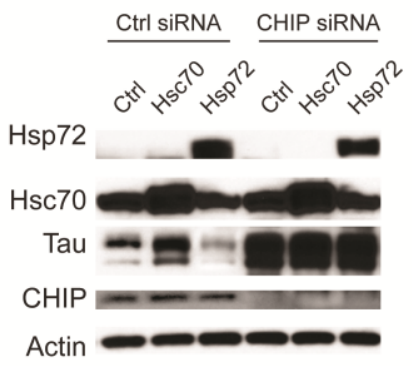

C.

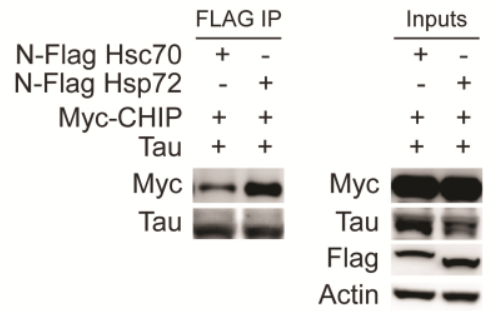

B.

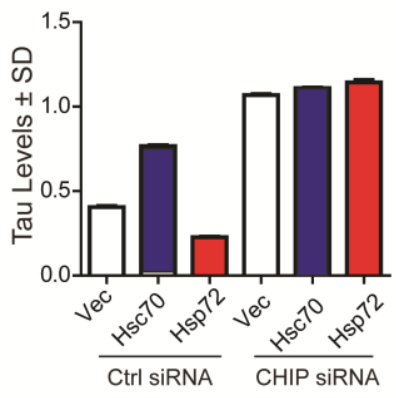

D.

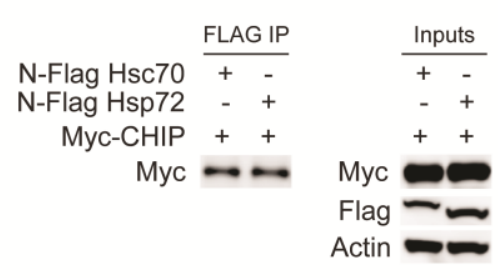

Figure 6: Tau influences the composition of Hsp70/CHIP complexes, affecting its own triage.

(A) Western blots of HeLa cells that were transfected with Hsp72, Hsc70, and siRNA for CHIP. (B) Quantification of tau levels in A shown as a ratio to Actin \pm standard deviation (SD) derived from 3 repeated experiments. Western blot of samples co-immunoprecipitated with anti-Flag antibody with (C) or without (D) tau over-expression shows that tau selectively increases the association of Hsp72 with CHIP.

To better understand the physiological relevance of these physical differences, we investigated the protein levels of Hsc70 and Hsp72 in brain homogenates from normal and Alzheimer's disease (AD) brains. Using a dot blot, we generated a standard curve for antibodies targeting Hsc70 and Hsp72 to account for differences in antibody affinity (Figs. 7A-C). We then spotted $20 \mu \mathrm{g}$ of total protein from AD and control brains onto dot blot membranes, probed with Hsp72 or Hsc70 antibodies, and plotted the resulting intensities along the standard curves generated with recombinant proteins (Figs. 7A-C). Relative quantitation of these proteins revealed that levels of Hsc70 were 30 times greater than Hsp72, with no difference between normal and AD brains (Fig 7D). Staining of mouse brain tissue produced similar results, revealing an excess of Hsc70 relative to Hsp72 (Fig. $7 \mathrm{E})$. 


\section{Imbalance of Hsp70 Family Variants Fosters Tau Accumulation}

A.

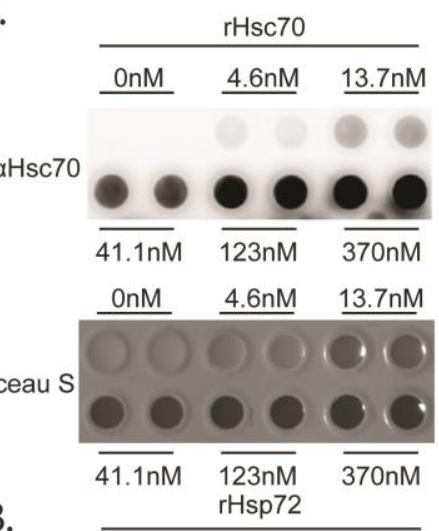

B.

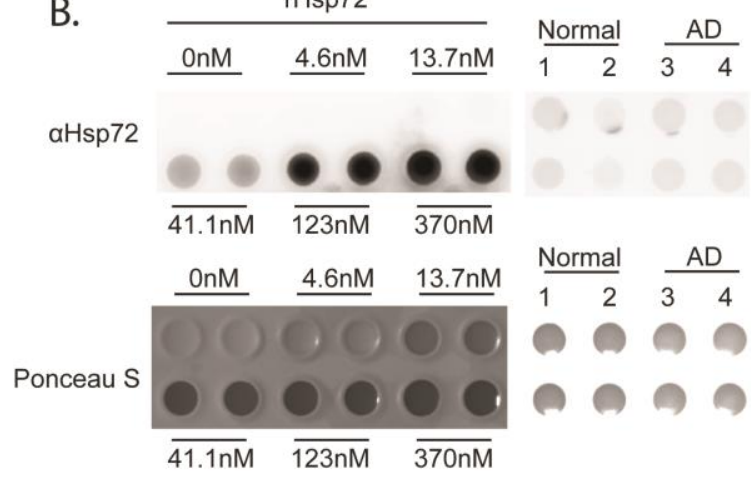

D.

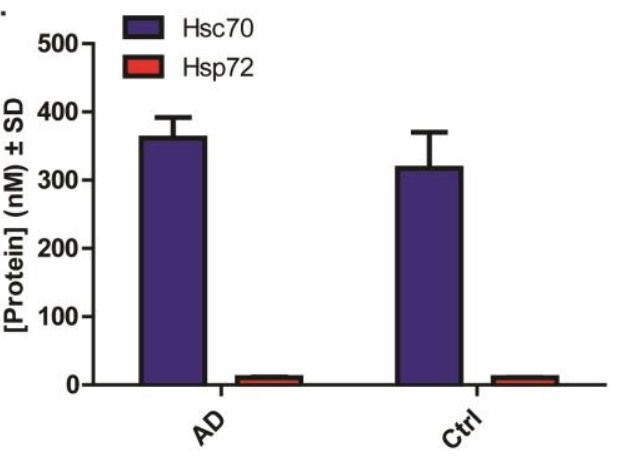

E.

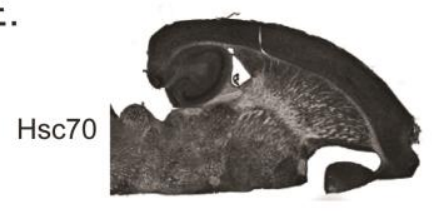

Hsp72

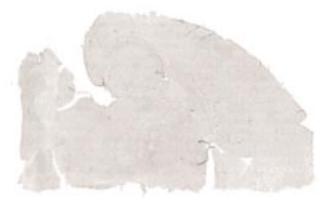

C.

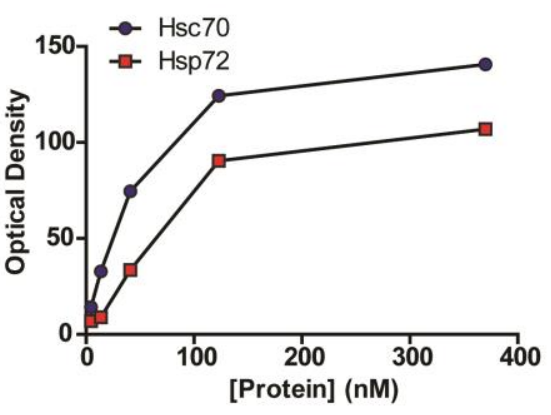

Figure 7: Hsc70 levels are much higher than Hsp72 in human brain.

Recombinant Hsc70 (A) and Hsp72 (B) were spotted onto nitrocellulose at indicated concentrations alongside $20 \mu \mathrm{g}$ of total protein from normal and AD brain lysates. Ponceau S was performed, followed by blocking and immunoblot with anti-Hsc70 or anti-Hsp72 antibodies. (C) Semi-log linear regression analysis was performed using the values of the recombinant protein to normalize for difference in antibody affinity. (D) Abundance of Hsc70 (blue bars) and Hsp72 (red bars) immunoreactivity in lysates from normal and Alzheimer's human brain ( $\mathrm{n}=3$ /group) normalized to the standard curve composed of recombinant Hsc70 and Hsp72, respectively in panels A and B. SD indicates standard deviation. (E) Representative images of immunostaining of mouse brain $(n=4)$ with Hsc70 and Hsp72 antibodies. 


\section{Imbalance of Hsp70 Family Variants Fosters Tau Accumulation}

\section{DISCUSSION}

We have found that Hsc70 and Hsp72 have opposing effects on tau that are driven by their relative expression levels, affinities for tau, and their C-terminal interaction domains. The data presented here suggest that the C-terminal dynamics of Hsc70 and Hsp72 are essential for whether tau degradation is accelerated or slowed. Because the Cterminal domains of these proteins form the lid of the substrate binding pocket and host interactions with co-chaperones that contain the TPR motif $(29,30)$, we predicted that levels of co-chaperones known to interact with the C-terminal portions of Hsp72 and Hsc70 could also have a major influence on tau triage decisions. One such TPR-containing protein, the carboxyl terminus of Hsc70-interacting protein (CHIP), has been extensively linked by our lab and others to both $\mathrm{Hsp} 70$ proteins and tau $(5,6,31,32)$. This protein has ubiquitin ligase activity and has been shown to facilitate tau clearance. Here we discovered that CHIP is able to bind to Hsc70 and Hsp72 differently when tau is present. This suggests that there are unique structural changes to both Hsp70 variants brought on by tau and other co-chaperones that can dictate CHIP association. Future studies will determine what these structures are and what components are needed; however these cellular findings point to this phenomenon as an important component for tau triage. Perhaps this represents a mechanism for tau to maintain its own proteostasis; as tau levels become over-abundant and exceed the capacity of the microtubule network, tau interacts more with Hsp72 and recruits a distinct sub-set of co-chaperones including CHIP to promote its self-destruction. This would prevent its accumulation inside neurons. However, this same recruitment of CHIP did not occur when tau interacted with Hsc70, suggesting that restoring or boosting the levels of Hsp72 may supplant Hsc70 and promote tau clearance. 


\section{Imbalance of Hsp70 Family Variants Fosters Tau Accumulation}

Interestingly, we also found that Hsp72 levels were not induced in Alzheimer's disease, despite the chronic stress conditions that must occur during the disease course. It is unclear why tau accumulation failed to promote more robust expression of Hsp72 in contrast to what has been reported for other neurodegenerative diseases brought on by protein misfolding and accumulation $(11,33)$. It is possible that Hsp72 levels are elevated early in the disease process, but then attenuate over time back to baseline levels due to the chronic proteotoxic stress. We would predict that even a small increase in Hsp72 could counter-balance tau burden by subverting the preservative effects of Hsc70 due to the enhanced affinity of Hsp72 for Hsc70, but not even slight increases were observed in human Alzheimer's or tau transgenic mouse brain tissue. This could be indicative of the failing heat shock response that occurs in general with age $(34,35)$. Thus, selective enhancement of Hsp72 levels with small molecules or gene therapies could favor an environment that removes excess tau.

Using NMR spectroscopy, we identified the binding motifs for a chaperone on tau. As expected, both Hsc70 and Hsp72 shared binding sites within the microtubule binding domains of tau. In particular, consistent with previous studies using traditional mutagenesis, the ${ }^{275}$ VQIINK ${ }^{280}$ and ${ }^{306}$ VQIVYK $^{311}$ hexapeptide motifs were found to be critical for binding these chaperones to tau, $(26,27)$. These motifs are particularly important for tau in that they are necessary for tau aggregation $(36,37)$ and triage towards an inefficient chaperone mediated autophagy (CMA) pathway (26). These pro-amyloid motifs reside in most proteins (38) and may serve as beacons for chaperones $(39,40)$. Thus, as tau disengages from microtubules, these motifs are exposed, recruiting available chaperones. It is at this point that the balance between Hsp72 and Hsc70 may be most critical. Hsc70 is necessary for CMA in that it facilitates the triage of certain proteins 


\section{Imbalance of Hsp70 Family Variants Fosters Tau Accumulation}

toward Lamp2a, a lysosomal membrane protein for CMA substrates (41); however Hsp72 does not have a similar function. While facilitating CMA would at first seem beneficial in promoting tau clearance, tau actually is anchored to the lysosomal membrane following its triage to Lamp2a, whereby it is then proteolytically cleaved and primed for aggregation (26). Using NMR, we were also able to identify some additional consensus sites ${ }^{346}{ }^{2 K D R V Q S K}{ }^{353}$ and ${ }^{375}$ KLTFRE $^{380}$ that were previously unidentified. Most intriguingly, the lysine at 353 is part of the known KXGS consensus sequence for the microtubule affinity regulating kinase 2 (MARK2). We previously demonstrated that CHIP is less prone to interact with tau when it is phosphorylated at MARK2 consensus sites (42-44). MARK2 promotes the release of tau from microtubules (45) while also altering tau sorting within neurons (46). Since tau phosphorylated at these sites is protected from CHIP interaction, it suggests that this is a strategy adopted by the neuron to preserve tau during plasticity to conserve energy. We speculate that to promote plasticity, tau must detach from microtubules - but without a post-translational modification of some sort, a CHIP/Hsp72 complex would quickly bind and promote tau clearance, a step that would require new tau protein to be synthesized after every axonal plastic event. By promoting phosphorylation of tau near the CHIP/Hsp72 binding sites, the neuron can preserve tau and conserve energy by allowing tau recycling: But this comes with a price; phosphorylation of tau destabilizes the axonal diffusion barrier that prevents tau localization to the soma and dendrites (46), the sub-cellular location where tau pathology is typically found in Alzheimer's disease and other tauopathies. Therefore, while preventing CHIP/Hsp72 binding through MARK2 phosphorylation may be energetically favorable for the neuron, it allows tau to move into the somatodendritic compartment where it is known to accumulate and become toxic. A delicate balance is required to meet energy demands while also preventing proteotoxicity. 


\section{Imbalance of Hsp70 Family Variants Fosters Tau Accumulation}

Expression levels of Hsp70 variants, tau phosphorylation, microtubule integrity, and neuronal trafficking all must be managed to prevent tau from accumulating to the point of being neurotoxic. If any of these processes are altered, the toxic sequelae of tau can be accelerated. Thus, interventions aimed at any of these pathways could be effective therapeutics for managing tauopathies.

\section{AUTHOR INFORMATION}

\section{Corresponding Authors}

Chad A. Dickey; 4001 E Fletcher Ave, Tampa, FL, 33613 Rm 325; Tel:+1 813396 0639; Fax: +1 8133960373 ;

Email: cdickey@health.usf.edu

\section{Running Title}

Hsp70 variants triage tau distinctly

\section{ACKNOWLEDGMENTS}

CAD was supported by grants from NIH/NIA (R00AG031291), NIH/NINDS (R01NS073899), the Alzheimer's Association and CurePSP. UKJ was supported by the Alzheimer's Association. JEG was supported by NIH/NINDS (NS059690) and the Alzheimer's Association.

\section{ABBREVIATIONS}

AD, Alzheimer's disease; CHIP, carboxy-terminus of Hsc70 interacting protein; CMA, chaperone-mediated autophagy; CTD, C-terminal domain; FBS, fetal bovine serum; FP, fluorescent polarization; GFP, green fluorescent protein;HEK, human embryonic kidney; Hsc, heat shock cognate; Hsp 70, heat shock protein; HSQC, heteronuclear single quantum 


\section{Imbalance of Hsp70 Family Variants Fosters Tau Accumulation}

coherence; LB; Luria-Bertani; MARK2, microtubule affinity regulating kinase 2; NBD, nucleotide binding domain; NMR, nuclear magnetic resonance; RFP, red fluorescent protein; SBD, substrate binding domain; Tet, tetracycline; TPR, tetratricopeptide repeat

\section{KEYWORDS}

Chaperones, CHIP, Hsc70, Hsp72, Tauopathies

\section{CONFLICT OF INTEREST}

The authors declare that they have no conflict of interest.

\section{REFERENCES}

1. Jinwal, U. K., O'Leary, J. C., 3rd, Borysov, S. I., Jones, J. R., Li, Q., Koren, J., 3rd, Abisambra, J. F., Vestal, G. D., Lawson, L. Y., Johnson, A. G., Blair, L. J., Jin, Y., Miyata, Y., Gestwicki, J. E., and Dickey, C. A. (2010) Hsc70 rapidly engages tau after microtubule destabilization. J Biol Chem 285, 16798-16805

2. Ebneth, A., Godemann, R., Stamer, K., Illenberger, S., Trinczek, B., and Mandelkow, E. (1998) Overexpression of tau protein inhibits kinesin-dependent trafficking of vesicles, mitochondria, and endoplasmic reticulum: implications for Alzheimer's disease. J Cell Biol 143, 777-794

3. Mattson, M. P. (1992) Effects of microtubule stabilization and destabilization on tau immunoreactivity in cultured hippocampal neurons. Brain research 582, 107 118

4. Brion, J. P., Octave, J. N., and Couck, A. M. (1994) Distribution of the phosphorylated microtubule-associated protein tau in developing cortical neurons. Neuroscience 63, 895-909 


\section{Imbalance of Hsp70 Family Variants Fosters Tau Accumulation}

5. Petrucelli, L., Dickson, D., Kehoe, K., Taylor, J., Snyder, H., Grover, A., De Lucia, M., McGowan, E., Lewis, J., Prihar, G., Kim, J., Dillmann, W. H., Browne, S. E., Hall, A., Voellmy, R., Tsuboi, Y., Dawson, T. M., Wolozin, B., Hardy, J., and Hutton, M. (2004) CHIP and Hsp70 regulate tau ubiquitination, degradation and aggregation. Hum Mol Genet 13, 703-714

6. Dickey, C. A., Yue, M., Lin, W. L., Dickson, D. W., Dunmore, J. H., Lee, W. C., Zehr, C., West, G., Cao, S., Clark, A. M., Caldwell, G. A., Caldwell, K. A., Eckman, C., Patterson, C., Hutton, M., and Petrucelli, L. (2006) Deletion of the ubiquitin ligase CHIP leads to the accumulation, but not the aggregation, of both endogenous phospho- and caspase-3-cleaved tau species. J Neurosci 26, 6985-6996

7. Dou, F., Netzer, W. J., Tanemura, K., Li, F., Hartl, F. U., Takashima, A., Gouras, G. K., Greengard, P., and Xu, H. (2003) Chaperones increase association of tau protein with microtubules. Proc Natl Acad Sci U S A 100, 721-726

8. Koren, I. I. I. J., Miyata, Y., Kiray, J., O'Leary, J. C., Nguyen, L., Guo, J., Blair, L. J., Li, X., Jinwal, U. K., Cheng, J. Q., Gestwicki, J. E., and Dickey, C. A. (2012) Rhodacyanine Derivative Selectively Targets Cancer Cells and Overcomes Tamoxifen Resistance. PloS one In Press

9. Goldfarb, S. B., Kashlan, O. B., Watkins, J. N., Suaud, L., Yan, W., Kleyman, T. R., and Rubenstein, R. C. (2006) Differential effects of Hsc70 and Hsp70 on the intracellular trafficking and functional expression of epithelial sodium channels. Proc Natl Acad Sci U S A 103, 5817-5822

10. Black, M. M., Chestnut, M. H., Pleasure, I. T., and Keen, J. H. (1991) Stable clathrin: uncoating protein (hsc70) complexes in intact neurons and their axonal transport. J Neurosci 11, 1163-1172

11. Williams, G. T., and Morimoto, R. I. (1990) Maximal stress-induced transcription from the human HSP70 promoter requires interactions with the basal promoter elements independent of rotational alignment. Mol Cell Biol 10, 3125-3136 


\section{Imbalance of Hsp70 Family Variants Fosters Tau Accumulation}

12. Tavaria, M., Gabriele, T., Kola, I., and Anderson, R. L. (1996) A hitchhiker's guide to the human Hsp70 family. Cell stress \& chaperones 1, 23-28

13. Qian, S. B., McDonough, H., Boellmann, F., Cyr, D. M., and Patterson, C. (2006) CHIP-mediated stress recovery by sequential ubiquitination of substrates and Hsp70. Nature 440, 551-555

14. Jinwal, U. K., Koren, J., 3rd, Borysov, S. I., Schmid, A. B., Abisambra, J. F., Blair, L. J., Johnson, A. G., Jones, J. R., Shults, C. L., O'Leary, J. C., 3rd, Jin, Y., Buchner, J., Cox, M. B., and Dickey, C. A. (2010) The Hsp90 cochaperone, FKBP51, increases Tau stability and polymerizes microtubules. J Neurosci 30, 591-599

15. O'Leary, J. C., 3rd, Li, Q., Marinec, P., Blair, L. J., Congdon, E. E., Johnson, A. G., Jinwal, U. K., Koren, J., 3rd, Jones, J. R., Kraft, C., Peters, M., Abisambra, J. F., Duff, K. E., Weeber, E. J., Gestwicki, J. E., and Dickey, C. A. (2010) Phenothiazine-mediated rescue of cognition in tau transgenic mice requires neuroprotection and reduced soluble tau burden. Mol Neurodegener 5, 45

16. Dickey, C., Kraft, C., Jinwal, U., Koren, J., Johnson, A., Anderson, L., Lebson, L., Lee, D., Dickson, D., de Silva, R., Binder, L. I., Morgan, D., and Lewis, J. (2009) Aging analysis reveals slowed tau turnover and enhanced stress response in a mouse model of tauopathy. Am J Pathol 174, 228-238

17. Desai, A., Verma, S., Mitchison, T. J., and Walczak, C. E. (1999) Kin I kinesins are microtubule-destabilizing enzymes. Cell 96, 69-78

18. Budde, P. P., Desai, A., and Heald, R. (2006) Analysis of microtubule polymerization in vitro and during the cell cycle in Xenopus egg extracts. Methods 38, 29-34

19. Mukrasch, M. D., Biernat, J., von Bergen, M., Griesinger, C., Mandelkow, E., and Zweckstetter, M. (2005) Sites of tau important for aggregation populate \{beta\}structure and bind to microtubules and polyanions. J Biol Chem 280, 24978-24986 


\section{Imbalance of Hsp70 Family Variants Fosters Tau Accumulation}

20. Delaglio, F., Grzesiek, S., Vuister, G. W., Zhu, G., Pfeifer, J., and Bax, A. (1995) NMRPipe: a multidimensional spectral processing system based on UNIX pipes. $J$ Biomol NMR 6, 277-293

21. Vranken, W. F., Boucher, W., Stevens, T. J., Fogh, R. H., Pajon, A., Llinas, M., Ulrich, E. L., Markley, J. L., Ionides, J., and Laue, E. D. (2005) The CCPN data model for NMR spectroscopy: development of a software pipeline. Proteins 59, 687-696

22. Abisambra, J. F., Blair, L. J., Hill, S. E., Jones, J. R., Kraft, C., Rogers, J., Koren, J., 3rd, Jinwal, U. K., Lawson, L., Johnson, A. G., Wilcock, D., O'Leary, J. C., Jansen-West, K., Muschol, M., Golde, T. E., Weeber, E. J., Banko, J., and Dickey, C. A. (2010) Phosphorylation dynamics regulate Hsp27-mediated rescue of neuronal plasticity deficits in tau transgenic mice. The Journal of neuroscience : the official journal of the Society for Neuroscience 30, 15374-15382

23. Ricci, L., and Williams, K. P. (2008) Development of fluorescence polarization assays for the molecular chaperone Hsp70 family members: Hsp72 and DnaK. Current chemical genomics 2, 90-95

24. Westerheide, S. D., Bosman, J. D., Mbadugha, B. N., Kawahara, T. L., Matsumoto, G., Kim, S., Gu, W., Devlin, J. P., Silverman, R. B., and Morimoto, R. I. (2004) Celastrols as inducers of the heat shock response and cytoprotection. $J$ Biol Chem 279, 56053-56060

25. Jo, H., Loison, F., Hattori, H., Silberstein, L. E., Yu, H., and Luo, H. R. (2010) Natural product Celastrol destabilizes tubulin heterodimer and facilitates mitotic cell death triggered by microtubule-targeting anti-cancer drugs. PloS one 5, e10318

26. Wang, Y., Martinez-Vicente, M., Kruger, U., Kaushik, S., Wong, E., Mandelkow, E. M., Cuervo, A. M., and Mandelkow, E. (2009) Tau fragmentation, aggregation and clearance: the dual role of lysosomal processing. Hum Mol Genet 18, 41534170 


\section{Imbalance of Hsp70 Family Variants Fosters Tau Accumulation}

27. Sarkar, M., Kuret, J., and Lee, G. (2008) Two motifs within the tau microtubulebinding domain mediate its association with the hsc70 molecular chaperone. $J$ Neurosci Res

28. Dolan, P. J., and Johnson, G. V. (2010) A caspase cleaved form of tau is preferentially degraded through the autophagy pathway. J Biol Chem 285, 2197821987

29. Rudiger, S., Buchberger, A., and Bukau, B. (1997) Interaction of Hsp70 chaperones with substrates. Nature structural biology 4, 342-349

30. Zhu, X., Zhao, X., Burkholder, W. F., Gragerov, A., Ogata, C. M., Gottesman, M. E., and Hendrickson, W. A. (1996) Structural analysis of substrate binding by the molecular chaperone DnaK. Science 272, 1606-1614

31. Ballinger, C. A., Connell, P., Wu, Y., Hu, Z., Thompson, L. J., Yin, L. Y., and Patterson, C. (1999) Identification of CHIP, a novel tetratricopeptide repeatcontaining protein that interacts with heat shock proteins and negatively regulates chaperone functions. Mol Cell Biol 19, 4535-4545

32. Sahara, N., Murayama, M., Mizoroki, T., Urushitani, M., Imai, Y., Takahashi, R., Murata, S., Tanaka, K., and Takashima, A. (2005) In vivo evidence of CHIP upregulation attenuating tau aggregation. J Neurochem 94, 1254-1263

33. Hammond, G. L., Lai, Y. K., and Markert, C. L. (1982) Diverse forms of stress lead to new patterns of gene expression through a common and essential metabolic pathway. Proc Natl Acad Sci U S A 79, 3485-3488

34. Fawcett, T. W., Sylvester, S. L., Sarge, K. D., Morimoto, R. I., and Holbrook, N. J. (1994) Effects of neurohormonal stress and aging on the activation of mammalian heat shock factor 1. J Biol Chem 269, 32272-32278

35. Heydari, A. R., Wu, B., Takahashi, R., Strong, R., and Richardson, A. (1993) Expression of heat shock protein 70 is altered by age and diet at the level of transcription. Mol Cell Biol 13, 2909-2918 


\section{Imbalance of Hsp70 Family Variants Fosters Tau Accumulation}

36. von Bergen, M., Friedhoff, P., Biernat, J., Heberle, J., Mandelkow, E. M., and Mandelkow, E. (2000) Assembly of tau protein into Alzheimer paired helical filaments depends on a local sequence motif ((306)VQIVYK(311)) forming beta structure. Proc Natl Acad Sci U S A 97, 5129-5134

37. von Bergen, M., Barghorn, S., Li, L., Marx, A., Biernat, J., Mandelkow, E. M., and Mandelkow, E. (2001) Mutations of tau protein in frontotemporal dementia promote aggregation of paired helical filaments by enhancing local beta-structure. J Biol Chem 276, 48165-48174

38. Goldschmidt, L., Teng, P. K., Riek, R., and Eisenberg, D. (2010) Identifying the amylome, proteins capable of forming amyloid-like fibrils. Proc Natl Acad Sci U S A 107, 3487-3492

39. Song, Y., Wu, Y. X., Jung, G., Tutar, Y., Eisenberg, E., Greene, L. E., and Masison, D. C. (2005) Role for Hsp70 chaperone in Saccharomyces cerevisiae prion seed replication. Eukaryot Cell 4, 289-297

40. Eisenberg, D., Nelson, R., Sawaya, M. R., Balbirnie, M., Sambashivan, S., Ivanova, M. I., Madsen, A. O., and Riekel, C. (2006) The structural biology of protein aggregation diseases: Fundamental questions and some answers. Acc Chem Res 39, $568-575$

41. Cuervo, A. M., and Dice, J. F. (1996) A receptor for the selective uptake and degradation of proteins by lysosomes. Science 273, 501-503

42. Dickey, C. A., Dunmore, J., Lu, B., Wang, J. W., Lee, W. C., Kamal, A., Burrows, F., Eckman, C., Hutton, M., and Petrucelli, L. (2006) HSP induction mediates selective clearance of tau phosphorylated at proline-directed Ser/Thr sites but not KXGS (MARK) sites. Faseb J 20, 753-755

43. Dickey, C. A., Kamal, A., Lundgren, K., Klosak, N., Bailey, R. M., Dunmore, J., Ash, P., Shoraka, S., Zlatkovic, J., Eckman, C. B., Patterson, C., Dickson, D. W., Nahman, N. S., Jr., Hutton, M., Burrows, F., and Petrucelli, L. (2007) The high- 


\section{Imbalance of Hsp70 Family Variants Fosters Tau Accumulation}

affinity HSP90-CHIP complex recognizes and selectively degrades phosphorylated tau client proteins. J Clin Invest 117, 648-658

44. Jinwal, U. K., Miyata, Y., Koren, J., 3rd, Jones, J. R., Trotter, J. H., Chang, L., O'Leary, J., Morgan, D., Lee, D. C., Shults, C. L., Rousaki, A., Weeber, E. J., Zuiderweg, E. R., Gestwicki, J. E., and Dickey, C. A. (2009) Chemical manipulation of hsp70 ATPase activity regulates tau stability. J Neurosci 29, 12079-12088

45. Drewes, G., Ebneth, A., Preuss, U., Mandelkow, E. M., and Mandelkow, E. (1997) MARK, a novel family of protein kinases that phosphorylate microtubuleassociated proteins and trigger microtubule disruption. Cell 89, 297-308

46. Li, X., Kumar, Y., Zempel, H., Mandelkow, E. M., Biernat, J., and Mandelkow, E. (2011) Novel diffusion barrier for axonal retention of Tau in neurons and its failure in neurodegeneration. EMBO J 30, 4825-4837 


\section{Appendix.}

\section{Calibration of PFG-Diffusion NMR}

(a) Representative table of standard proteins with their reported and measured hydrodynamic radii. The proteins were used to establish a (b) calibration curve for the PFG-diffusion NMR experiments.

a)

\begin{tabular}{lcccccc} 
Protein & Molecular Weight & Residues & Reported $\mathbf{R}_{\mathrm{g}}$ & Calculated $\mathbf{R}_{\mathrm{H}}$ & ${\text { Reported } \mathbf{R}_{\mathrm{H}}}^{\text {Experimental D }}$ \\
\hline Cytochrome C & $12.4 \mathrm{kDa}$ & 104 a.a & $13.51 \AA$ & $17.43 \AA$ & $17.82 \AA$ & $7.63 \times 10^{-11}$ \\
Lysozyme & $14.3 \mathrm{kDa}$ & 129 a.a & $15.32 \AA$ & $19.36 \AA$ & $20.51 \AA$ & $6.67 \times 10^{-11}$ \\
Myoglobin & $18.1 \mathrm{kDa}$ & 153 a.a & $18.56 \AA$ & $23.24 \AA$ & $21.24 \AA$ & $5.98 \times 10^{-11}$ \\
Ovalbumin & $45.2 \mathrm{kDa}$ & 385 a.a & $22.61 \AA$ & $28.66 \AA$ & $30.48 \AA$ & $4.35 \times 10^{-11}$
\end{tabular}

b)

Ln( Hydrodynamic Radius)

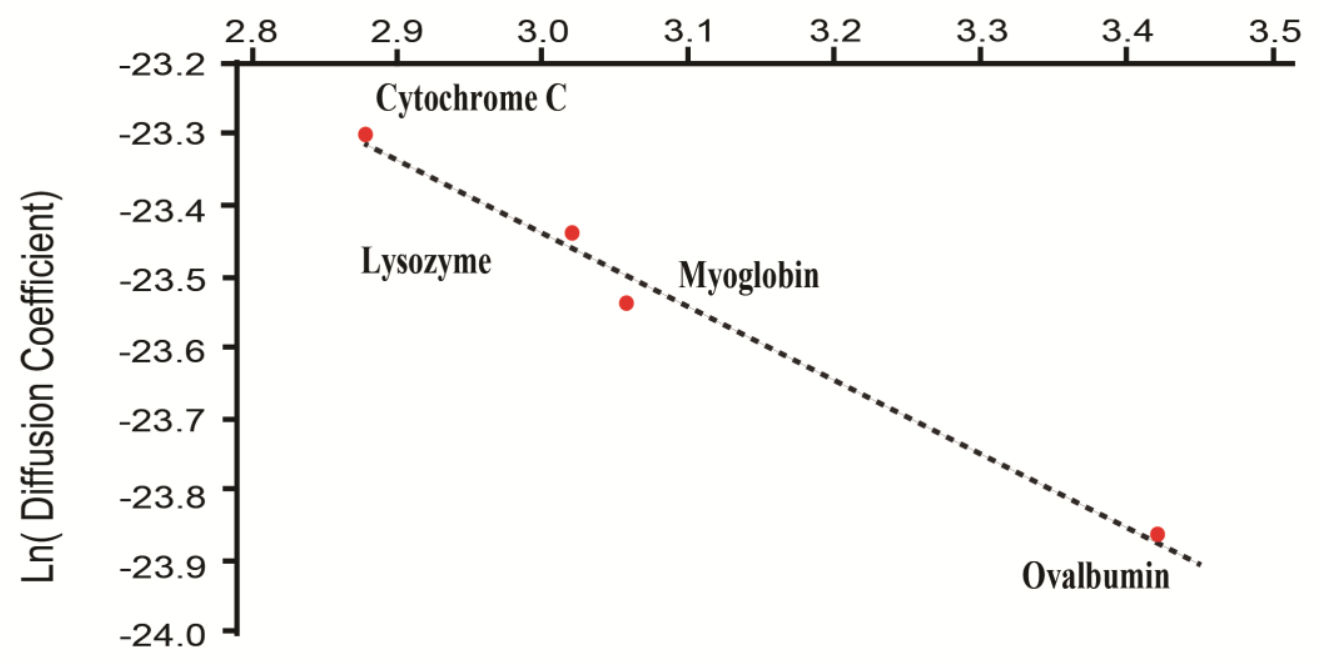




\section{Curriculum Vitae}

\section{Curriculum Vitae}

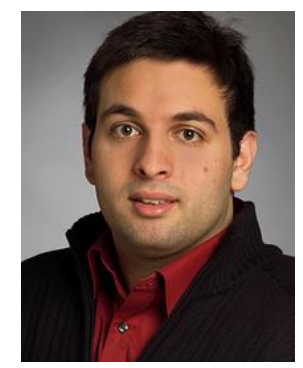

$2010-2013$

Doctoral Thesis Project: Tau Protein: Enhancement and Inhibition of Aggregation Institution: Max Planck Institute for Biophysical Chemistry, Göttingen, Germany Department: NMR-based Structural Biology Supervision: Prof. Dr. Markus Zweckstetter

Graduate School: GGNB, Georg-August Universität, Göttingen, Germany

$2008-2009$

Master Program: Advanced Spectroscopy in Chemistry

Master Thesis Project: Residual Dipolar Couplings in Organic Molecules using Lanthanide Tags

Institution: Faulty of Chemistry, Universität Leipzig, Germany

Department: Institute of Analytical Chemistry

Supervision: Prof. Dr. Stefan Berger

$2007-2008$

Master Program: Advanced Spectroscopy in Chemistry

Institution: Université des Sciences et Technologies de Lille, France

Department: UFR de Chimie - ENSCL

Supervision: Prof. Dr. Jean-Pierre Wignacourt

$2003-2007$

Undergraduate Studies: Bachelors in Chemistry

Institution: Lebanese University, Faculty of Sciences, Beirut Lebanon

Bachelors Thesis Project: Toxicity in Wine by Atomic Absorption Spectroscopy

Supervision: Dr. Hala Hafez

Email: ea-chemistry@hotmail.com

Website: http://eliasakoury.wix.com/eliasakoury 